1968

\title{
Mechanical Properties of Arteries
}

\author{
Philip Boone Dobrin \\ Loyola University Chicago
}

Follow this and additional works at: https://ecommons.luc.edu/luc_diss

Part of the Medicine and Health Sciences Commons

\section{Recommended Citation}

Dobrin, Philip Boone, "Mechanical Properties of Arteries" (1968). Dissertations. 924.

https://ecommons.luc.edu/luc_diss/924

This Dissertation is brought to you for free and open access by the Theses and Dissertations at Loyola eCommons. It has been accepted for inclusion in Dissertations by an authorized administrator of Loyola eCommons. For more information, please contact ecommons@luc.edu. (c) $($ ) $\Theta \Theta$

This work is licensed under a Creative Commons Attribution-Noncommercial-No Derivative Works 3.0 License. Copyright (C) 1968 Philip Boone Dobrin 
MECHANICAL PROPERTIES OF ARTERIES

by

PHILIP BOONE DOBRIN

A Thesis Submitted to the faculty of the Graduate School of Loyola University of Chicago in Partial Fulfillment of the Requirements for the Degree of Doctor of Philosophy

JUNE

1968 


\section{LIFE}

Philip 8. Dobrin was born in Passaic, New Jersey, on December 21, 1934.

He attended Lafayette College and New York University where he received the Bachelor of Arts degre日 in 1958.

After graduating, he was employed by the Charles

Pfizer Research Laboratories as a Research Assistant in Psychopharmacology. At the same time he attended Connecticut College, and in 1962, he received a Master of Arts degree in experimental psychology.

In 1963, he was accepted as a graduate student by the Physiology Department of Loyola University, Stritch School of Medicine, where he began training under the supervision of Dr. Allen A. Rovick. His work has been supported by a National Institutes of Health Predoctoral Fellowship, which he was awarded in 1965. 
ACKNOWLEDGEMENTS

The author is greatly indebted to several people. These include Dr. Allen A. Rovick and Dr. James Doyle for their expert and intellectual advice; Mrs. Gale Vance and Mrs. Eleanor Wilde for their technical assistance; Miss Carol Ofenloch and Miss Jeff Newman, who typed this imanuscipt; Dr. Walter Randall and my wife, Louella, both of whom have encouraged me greatly over the past years of study.

111 


\section{TABLE OF CONTENTS}

Chapter

I. INTRODUCTION $\ldots \ldots \cdots \cdots \cdots \cdots \cdots \cdots \cdots \cdots \cdots \cdots \cdots \cdots \cdots \cdots \cdots \cdots \cdots \cdots \cdots \cdots \cdots \cdots$ 1

II. REVIEW OF THE LITERATURE

A. Morphological Appearance.................. 2

B. Connective Tissue of the Arterial Wall

1. Elastin.......................... 14

2. Collagen........................ 32

3. Ground Substance..................44 44

C. Cellular Components of the Arterial Wall

1. Endothelium.....................45

2. Fibroblasts.....................4 48

3. Smooth musclo................... 50

D. Mechanical Properties of Arteries..........75

III. MATERIALS AND METHODS

A. Theory............................... 95

B. Apparatus and techniques................. 109

C. Procedure.............................129

IV. RESULTS

A. Wall Volume..........................140

B. Actions of NEpI and KCN ................ 142

C. Fixed-lengh Arteries.................... 155

D. Extension Behavior..................... 159

E. Hysteresis..........................175

F. Longitudinal Properties................. 189 
Chapter

V. DISCUSSION

A. Wall Volume....................... 199

B. Actions of NEpi and KCN................. 202

C. Specificity of NEpi and KCN............. 205

D. Shortening Behavior.................. 211

E. Distention Behavior................... 224

F. Longitudinal Properties................ 232

VI. SUMMARY AND CONCLUSIONS................... 251

BIBLIOGRAPHY........................... 253 
LIST OF TABLES

Table

Page

I. Action of KEpi and KCN on Arteries Subjected to Various Pretreatment Procedures

150

II. Shortening characteristics of NEpi-Activated

Carotid Arteries

162

III.A Elastic Modulus as a Function of Strain ............ 168

III.8 Elastic Modulus as a Function of Pressure ......... 169

vi 


\section{LIST OF FIGURES}

Figure

1. VESSEL SEGMENTS ILLUSTRATING MATHEMATICAL ANALYSIS $\ldots \ldots \ldots \ldots \ldots \ldots \ldots \ldots \ldots \ldots$

2. CURVED, 2-DIMENSIONAL SHEETS ILLUSTRATING CIRCUMFERENTIAL-LONGITUDINAL STRAINS ........... 103

3. APPARATUS USED TO MAINTAIN ARTERIES AT IN SITU LENGTH ............................ 111

4. APPARATUS USED TO MEASURE LONGITUDINAL FORCE ...... 113

5. DISPLACEMENT TRANSDUCER ................... 115

6. LINEARITY OF DISPLACEMENT TRANSDUCER ........... 118

7. FREQUENCY RESPONSE OF DISPLACEMENT TRANSDUCER $\ldots \ldots \ldots 121$ 8.A X-RAYS OF AN ARTERY; 16.5 MAGNIFICATION .......... 126 8.8 X-RAYS OF AN ARTERY; $100 \times$ MAGNIFICATION ......... 127 9. COMPARISON BETWEEN TRANSDUCER AND X-RAY MEASUREMENTS OF IDENTICAL ROD-SHAPED OBJECTS .............. 128

10. ARTERIAL WALL VOLUME ...................... 141

11. POLYGRAPH RECORDS ILLUSTRATING CONTRACTILE EFFECTS OF NEPI, AND DILATION PRODUCED BY KCN ........... 143

12. A NEPI DOSE-RESPONSE RELATIONSHIP ................. 144 12.8 KCN DOSE-RESPONSE RELATIONSHIP ............... 145 13. TIME COURSE OF KCN DILATION IN 5 ARTERIES ......... 147 14. COMPARISON OF ARTERIAL WALL VOLUME AFTER NEPI, AND AFTER KCN ................................ 152 
Figure

15. ARTERIAL WALL VOLUME AFTER NEPI, AND AFTER KCN ..... 154

16. SAMPLE POLYGRAPH RECORDS .................... 156

17. A ARTERY 1 \& PRESSURE RADIUS CURVES .............. 157

18. A ARTERY 2 \& PRESSURE RADIUS CURVES .............. 158

17.8 ARTERY I\& SHORTENING STRESS-STRAIN CURVES ......... 160

18.8 ARTERY 28 SHORTENING STRESS-STRAIN CURVES ........161

17.C ARTERY 1 \& DISTENTION STRESS-STRAIN CURVES ........ 164

18.C ARTERY 28 DISTENTION STRESS-STRAIN CURVES $\ldots \ldots \ldots \ldots \ldots 165$

17.D ARTERY I: STRAIN ELASTIC MODULUS CURVES FOR

DISTENTION BEHAVIOR $\ldots \ldots \ldots \ldots \ldots \ldots \ldots \ldots \ldots \ldots 166$

18.D ARTERY 28 STRAIN ELASTIC MODULUS CURVES FOR

DISTENTION BEHAVIOR ................. 167

17.E ARTERY $1:$ PRESSURE-ELASTIC MODULUS CURVES FOR

DISTENTION BEHAVIOR $\ldots \ldots \ldots \ldots \ldots \ldots \ldots \ldots \ldots . . \ldots 171$

18.E ARTERY 2: PRESSURE-ELASTIC MODULUS CURVES FOR

DISTENTION BEHAVIOR $\ldots \ldots \ldots \ldots \ldots \ldots \ldots \ldots \ldots . . . . .172$

19. MEAN ELASTIC MODULUS AND STANDARD ERRORS AS A FUNC-

TION OF PRESSURE; EFFECTS OF NEPI AND KCN ARE COM-

PARED WITH THE UNTREATED ARTERIES OF BERGEL $\ldots \ldots \ldots \ldots .174$

20. PRESSURE-RADIUS SHORTENING CURVES FOLLOWING INTERRUPTION OF DISTENTION SEQUENCES $\ldots \ldots \ldots \ldots \ldots \ldots \ldots \ldots \ldots \ldots .176$

21. A ARTERY 38 PRESSURE-RADIUS SHORTENING CURVE BEGUN FROM VARIOUS ORIGINS $\ldots \ldots \ldots \ldots \ldots \ldots \ldots \ldots \ldots . .178$

21.B ARTERY 3:- STRESS-STRAIN CURVES FOR SHORTENING SEQUENCES BEGUN FROM VARIOUS ORIGINS .... 179

22. A ARTERY $4:$ PRESSURE-RADIUS SHORTENING CURVES

BEGUN FROM VARIOUS ORIGINS $\ldots \ldots \ldots \ldots \ldots \ldots \ldots 180$ 
Figure

22.8 ARTERY $4:$ STRESS-STRAIN CURVES FOR SHORTENING

SEQUENCES BEGUN FROM VARIOUS ORIGINS ..... 181

23. A ARTERY 58 PRESSURE-RADIUS DISTENTION CURVES

BEGUN FROM VARIOUS ORIGINS $\ldots \ldots \ldots \ldots \ldots \ldots 183$

24. A ARTERY 58 PRESSURE-RADIUS DISTENTION CURVES

BEGUN FROM VARIOUS ORIGINS ............ 184

23.8 ARTERY 58 STRESS-STRAIN, CURVES FOR DISTENTION

SEQUENCES BEGUN FROM VARIOUS ORIGINS $\ldots \ldots 185$

24.8 ARTERY 68 STRESS-STRAIN CURVES FOR DISTENTION

SEQUENCES BEGUN FROM VARIOUS ORIGINS .... 186

23. C ARTERY 58 STRAIN-ELASTIC MODULI CURVES FOR DIS-

TENTION SEQUENCES BEGUN FROM VARIOUS

ORIGINS ......................... 187

24.C ARTERY 6: STRAIN-ELASTIC MODULI CURVES FOR DIS-

TENTION SEQUENCES BEGUN FROM VARIOUS

ORIGINS ....................... 188

25. POLYGRAPH RECOROS ILLUSTRATING LONGITUDINAL FORCE

MEASUREMENTS ............................ 190

26. A ARTERY 78 LONGITUDINAL STRESS-CIRCUMFERENTIAL

STRAIN CURVE ....................... 193

27. A ARTERY $8:$ LONGITUDINAL STRESS-CIRCUMFERENTIAL

STRAIN CURVE ..................................... 194

26.B ARTERY 7 : PLOT OF CIRCUMFERENTIAL ELASTICITY

AGAINST LONGITUDINAL ELASTICITY ........ 197

27.8 ARTERY $8:$ PLOT OF CIRCUMFERENTIAL ELASTICITY

AGAINST LONGITUDINAL ELASTICITY ........ 198

28. MECHANICAL MODELS OF THE ARTERIAL WALL ......... 226

29. PRESSURE-RADIUS CURVES FOR DISTENTION SEQUENCES IN AN ARTERY MAINTAINED WITHIN A RESTRICTED RANGE. OF RADII 
Figure

30. A CIRCUMFERENTIAL AND LONGITUDINAL STRESS-STRAIN CURVES FOR SHORTENING SEQUENCES IN A FREELY DISTENSIBLE ARTERY .............................249

30.8 CIRCUMFERENTIAL AND LONGITUDINAL ELASTIC MODULUSSTRAIN CURVES FOR DISTENTION SEQUENCES $\ldots \ldots \ldots \ldots \ldots \ldots \ldots .250$ 


\section{CHAPTER I}

INTRODUCTION

Large conduit arteries convey blood from the heart to the distributing vessels of the peripheral tissues. In fulfilling this function, the conduit arteries play three important roles: 1) they damp flow oscillations by serving as transient blood stores, filling during systole and draining during diastole; 2) they damp the pressure oscillations by serving as transient energy stores, distending duringsystole and recoiling during diastole; 3 ) they transmit thepressure pulse, a parameter of physiological and clinical concorn.

Much experimental information has been obtained regarding the arterial wall. In most of the physlological studies, vascular strips, rings, or isolated pressure-volume segments have been used; in pharmacological studies, spiral strips cut from the muscular media have usually been used: These preparations differ from in situ conditions in that they fail to maintain appropriate geometry, and in many cases, ignore the mechanical properties of the passive arterial connective tissue. The wall, in its entirety, must be considered.

The present research is directed toward a more precise description of the physical characteristics of the arterial wall, and is especially concerned with the way in which these characteristics are altered by the presence of active smooth muscle. 


\section{REVIEW OF THE LITERATURE}

A. Morphological Appearance. Light microscope studies of the walls of arteries reveal three falrly distinct, concentric layers; an inner intima bordering the lumen, a media, and an outermost adventitia (Benninghoff, 1927).

1. Intima. The intima is composed of a single layer of endothelial cells. These are separated from the media by the internal elastic lamina. Endothelial cells of the rabbit aorta have been described as $30-35 \mu$ by $8-15 \mu$ when fixed in the retracted state (Ferrara, 1950). They are flat except in the nuclear region where they may be wider, in order to accomodate the nucleus. In undistended arteries, the endothelial cells often bulge into the lumen (Buck, 1958; VanCitters et al, 1962; Hayes, 1967). Upon examination under the light microscope, the endothelium appears to form a smooth, flat sheet when it is distended by physiological pressures. However, electron microscopy reveals that small endothelial processes may still project into the lumen, even when the vessel is moderately distended (Matthews and Gardner, 1966).

Buck (1958) and Pease and Paule (1960) observed by electron microscopy that an interphase of collagenous fibers is located between the endothelium and the internal elastic lamina, although other authors claim that the endothelium rests 
directly upon the internal elastic lamina (Policard et al, 1955, 1960; Karrer, 1958). The mechanical influence of collagenous fibers in this location could be important, but this would depend upon whether they were in a slack or stretched state at physiological pressures. Endothelial cells are bounded by a three-layered membrane which often manifests tortuous folds, even in the distended artery. Vesicles may be seen within the endothelial cell cytoplasm, usually near the membrane. The vesicles are usually interpreted as inpocketings of the cellular membrane (Rhodin, 1962).

2. Media. The media of the arterial wall is composed of alternating layers of elastic lamina and smooth muscle cells. The elastic lamina are composed of many fine, homogenous fibers. These fibers vary in size, and appear to run randomly in all directions (Dees, 1923; Ayer and Eisenstein, 1963). A tangle of microfibrils may be seen near and tttached to strands of elastin (Karrer, 1961). Some of these are as small as $80 \AA$ in diameter (Low, 1962). Cross-sections of the lamina of retracted arteries show many dots indicating some longitudinal disposition of elastic fibers (Huber, 1916). The gross appearance of the lamina is one of thick, corrugated lines. However, this corrugated appearance is an artifact of vessel rotraction prior to fixation. If vessel distention is maintained during fixation, these corrugations are not seen. Instead, the elastic lamina are seen as smooth, thin, concentric 
lines (Wolinsky and Glagov, 1964; Bunce, 1965; Hayes, 1967). Occasional breaks are seen in the lamina, which may be identified as gaps or windows (lamina fenestrata) when viewed in longitudinal sections. Fine elastic fibers also form a net, spanning successive concentric elastic laminae. Thus, a threedimensional elastin frame provides a netusork throughout the media. At physiological pressures these fine spanning fibers are pulled into a nearly circumferential position (Wolinsky and Glagov, 1964).

In large elastic arteries, smooth muscle cells bridge successive elastic lamina, forming so-called "spanmuskeln" (Benninghoff, 1927). The muscle is usually attached directly to the main body of the elastic lamina, although occasionally the muscle may be attached to a stray tail, presumably of elastin, projecting from the lamina (Keech, 1960).

In electronmicrographs the muscle cells appear to be attached to elastin by a $200 \AA$ layer of cement substance (Pease and Paule, 1960). Strong(1938) discovered during microdissection of arteries that the media can be unravelled as a spiral strip. Longitudinal muscle was found only in the femoral artery, and even there, it was sparse as compared with the abundant circumferentially-oriented muscle. Analyses of arterial sections cut at many different angles led Fischer(1951) and Coerttler (1953) to conclude that the circular muscle is arranged in continuous spirals within the media. The presence 
of left and right spirals probably explains how contraction produces a decrease in diameter with no gross twisting of the vessel.

In the relaxed state, smooth muscle cells are long, thin, and spindle-shaped, about 60ر long and $2 \mu$ wide. The nucleus is usually in the center of the cell. McGill(1909) noted that mechanical and drug-induced smooth muscle contraction caused no changes in the volume of the nucleus, although it did cause wrinkling of the nuclear outline. Other cyto. plaṣm organelles are usually near the longitudinal poles of the cells. Myofilaments of uniform size appear to run throughout the cells. Successive smooth muscle cells are staggered so that the wide central portion of one cell lies adjacent to the narrow, end-region of another. Individual cells have a threelayered membrane, and it is generally conceded that the plasma membrane is normal in appearance. The basement membrane, however, is controversial. Rhodin(1962) working with the fem-. oral artery, and Matthews and Gardner (1966) working with the mesenteric artery, observed well-defined basement membranes, while Pease and Paule(1960) using the aorta, and Pease and Molinari (1960) studying pial arteries observed a rudimentary basement membrane. The latter authors noted similar reports of a poorly developed basement membrane by Caeser ot al (1957) in the smooth muscle of urinary bladder and uterus, and by Mark 
(1956) who also studied uterus. Evans and Evans (1964) compared the non-innervated amnionic smooth muscle with the innervated nictitating membrane muscle in the chick embryo. An interesting correlation was observed between the consistent presence of a basement membrane in the nictitating membrane (a multi-unit muscle receiving a high ratio of nerve-to-muscle innervation, and the absence of a basement membrane in the amnionic muscle which is totally non-innervated. These observations suggest that the absence of a basement membrane may be related to excitation by diffusion of neurohumoral stimuli, or to a cell-to-cell mode of transmission of excitation. Intercellular bridges between smooth cells of arteries have not been observed, although Parker(1958) has observed close contact between adjacent cells in coronary arteries. In large elastic arteries an intercellular space is present essentially excluding intercellular contact. In fact, in some arteries a layer of collagenous or elastic fibers has been seen surrounding the individual smooth muscle cells. (Movat and Fernando,1963).

The media also contains many collagen fibrils. These are randomly dispersed in retracted vessels, but exhibit circumferential orientation in vessels distended to $150 \mathrm{~mm} \mathrm{Hg}$ (Wolinsky and Glagov, 1964). Collagen fibrils are not connected to either elastic or muscular elements (Pease and Paule, 1960; Pease and Molinari, 1960; Wolinsky and Glagov, 1964). 
Anatomical studies thus indicate that the elastin and smooth muscle are essentially in parallel, and that these elements, in turn, are in parallel with collagen.

3. Adventitia. The outermost layer of the arterial wall is the ooventitia. It is composed of elastin, many collagen fibrils, and numerous long, thin tapered fibroblasts. All but the neural elements are arranged circumferentially. Wolinsky and Glagov(1964) fixed rabbit aortae while under various distending pressures. They reported that unlike medial connective tissue fibers, the adventitial elastin and collagen were neither markedly wrinkled at low pressure, nor markedly straightened at high pressures. These authors concluded, therefore, that it is the medial collagen which provides the relative inextensibility at pressures above $80-100 \mathrm{~mm} \mathrm{Hg}$. Since the adventitial collagen remains somewhat slack, even at $200 \mathrm{~mm} \mathrm{Hg}$, this component is thought to provide a protective jacket against extremely high distending pressures (Burton, 1954).

4. Vasa Vasora. the vasa and vena vasorum provide the arterial wall with nutrition and removal of waste products. The vasa vasorum usually derives from branches of the artery in question, and also from adjacent arteries. The carotid, for example, is supplied by variable sources but almost always includes small branches which arise from the carotid itself. 
Often a branch derived from the axial artery is also present (Vio et al, 1964). A vena vasorum usually runs with the vasa vasorum and, in the case of the carotid, empties into the jugular vein (Vio et al, 1964). Extensive studies on thé aorta and coronary arteries indicate that arterial vasa vasorum entering from the adventitia of arteries break up into arterioles and capillaries, but that these are distributed only in the outer two-thirds of the media in normal vessels (Paterson, 1936; leary, 1938; Horn and Finklestein, 1940; Nelson, 1941; Schlichter, 1949; Wartman, 1950; Geiringer, 1951). It is not surprising that patent arterioles and capillaries are not found in the inner third to half of the wall; the predicted intramural stress (Peterson, 1966) is highest at the lumenal margin, declining rapidly across the wall. It is lowest at the adventitial margin. Interestingly, all of the many anatomical studies mentioned above indicate that capillaries are found in the intima only where sclerotic thickening is ob served. This implies that either increased diffusion distances somehow induce the development of intimal vascularization to fulfill metabolic needs, or that the presence of atheromatous plaques and other such lesions provide sufficient rigidity to prevent compression of intimal vasa vasora. Studies on the diffusion of various solutes through the wall of excised vessels (Woerner, 1951; Wilens and McCluskey, 1952; 
Kirk and Laursen, 1955) indicate that many water-soluble and lipid-soluble substances Ieadily penetrate the intima and media. The adventitia tends to be. much less permeable than the inner regions, especially to lipids. Barcroft et al (1944) presented evidence that mucopolysaccharides may play a role in facilitating diffusion of nutrient substances in the umbilical cord. The mucopolysaccharide ground substance in arteries may play a similar role in providing passage where no discrete vasa vasorum exist. Shimamoto (1961) identified a large subendothelial space which presumably is fluid-filled in life, and which could provide a channel through which nutrient and metabolic materials might flow and diffuse.

Quantitative studies on bulk flow of lumenal fluids

through the vasa vasorum have been undertaken by Jaeger (1964). Isolated vessels were mounted in a tissue bath which was sealed except for a vertical pipette. Tho vessels were stretched, sealed at one end, and the lumen filled with fluid. A catheter connected to the unsealed end of the vessel permitted oscillating pressures to be imposed upon the lumenal fluid. Changes in the height of the extravascular fluid in the pipette were used to measure transmural flow. Pig carotids exhibited flows of 0.5 to $0.6 \mathrm{ml}$ Tyrode solution per minute per $100 \mathrm{gm}$ of tissue, while dog carotids exhibited flows of 0.3 to $0.4 \mathrm{ml}$ Tyrode solution per minute per $100 \mathrm{gm}$ of tissue. According 
to the author, these values are about four times as high as more viscous blood. However, no consideration was given to the difference in oxygen-carrying capacities between Tyrode's solution and blood, or to any metabolic autoregulation of vasa vasorum that might occur. Transmural flow increased rapidly up to $150 \mathrm{~cm} \mathrm{H} \mathrm{H}_{2} \mathrm{O}$ transmural pressure, then increased gradually above this value. Flow tended to decrease with muscle contraction and also with age. India ink studies indicated that the flow may take two routes. One path is by the vasa externa, vessels which orlginate at the lumen, pass through the wall and turn back to reenter the adventitia. Another path is by the vasa interna, vessels which supply the wall directly from the lumen. The ostia for the vasa externa appear in the walls of side branches off the parent artery, while the ostia for the vasa interna appear in smooth portions of the arterial endothelium. Tying all visible external vessels reduces the total transmural flow to dout $25 \%$ of the original value, so that the vasa interna and diffusion accounts for about a quarter of total transmural flow.

5. Innervation. Blood vessels receive both afferent and efferent innervation. Polley(1955) demonstrated by degeneration studies that blood vessels in skeletal muscle and skin receive visceral afferent innervation. These fibers terminate in the perivascular connective tissue in the adventitia. 
Even in the carotid sinus and the common carotid baroreceptor areas, regions rich in afferent innervation, light microscopy studies reveal that afferent fibers terminate only in the adventitia (deCastro, 1928; Abraham, 1949; Boss and Green, 1956). Presumably other afferent fibers are similarly localized in the wall of arteries. With the exception of a fow areas of the body, efferent fibers to the arterial wall are entirely sympathetic. Much research using light microscopy in conjunction with methylene blue and silver staining has been published describing the innervation of smooth muscle. It is generally agreed that the terminal portion of autonomic fibers end in a fine, meshed plexus of unmyelinated fibers 0.1 to $1.7 \mathrm{w} \mathrm{in}$ diameter. This plexus has been called an "Autonomic ground plexus" (Hillarp, 1959) and a "Sympathetic ground plexus" (Booke, 1949).

Electronmicroscopy studies of the innervation of the arterial wall have also been reported. With few exceptions, these studies indicate that all the innervation terminates somewhere in the adventitia, usually near the adventitio-medial border (Caeser et al, 1957; Keech, 1960; Samarsinghe, 1963; Dahl and Nelson, 1964). Parker (1958) noted that often several unmyelinated axons may be enclosed by a single Schwann cell. Zelander et al (1962) and Appenzeller (1964) reported that abundant numbers of vesicles may be present in the axons in- 
nervating blood vessels. These vesicles appeared to be most concentrated at regions of the neurons nearest the smooth muscle cells. Presumably these vesicles contain the granules seen in sympathetic axons and which Wolfe et al (1962) demonstrated, by electronmicroscopic autoradiography, contain catecholamines. No specialized nerve-smooth muscle junction comparable to the skeletal muscle end-plate has been described. Yamamoto (1960) reported that axons lose their Schwann cell sheaths just before reaching the smoth muscle, and then actually penetrate smooth muscle cells. This is contrary to every other report. Close membrane-to-membrane contacts, however, have been observed. Nerves have been found very close to outpocketings of smooth muscle cells which protrude into the adventitia (Appenzeller, 1964; Dahl and Nelson, 1964; Lever et al, 1965). The junctional space has been described to be as small as $0.1 \mathrm{\mu}$. In the rat mesenteric artery (Matthews and Gardner, 1966) and as large as $0.5 \mathrm{w}$ in rabbit pulmonary artery. The outpocketings of smooth muscle cells also contain numerous vesicles and mitochondria, suggesting some important metabolic function.

\section{Extensive analyses of catecholamine content by} Schmiterlow (1948) also support the restriction of adrenergic fibers to the adventitia. Bioassay and chemical tests of bovine and equine carotid arteries indicated that almost all of 
the norepinephrine, $0.75 \mathrm{~N}$ per gram of fresh tissue, was confined to the adventitia.

The introduction of the histochemical fluorescence method of Falck and Hillarp (Falck, 1962; Falck ot al, 1962) has provided a new tool for evaluating the presence of postganglionic sympathetic neurons in arteries. When tissues are treated with formaldehyde gas, and then dehydrogenated, certain biogenic monoamines - such as norepinephrine, dopamine and 5-hydroxytryptamine - become visibly fluorescent. Swedish investigators (Norberg and Hamberger, 1964) demonstrated that adrenergic fibers are located in elastic arteries only as a thin meshwork layer just outside the media and along the vasa vasorum. This meshwork sends processes which project centripetally toward the muscle layers; however the nerve processes never penetrate among the muscle cells. Fluorescence tends to be most intense at the terminal regions of these apparant neuronal projections. Several lines of evidence indicate that this fluorescence fades beginning about three hours after reserpinization, first in the nerve cell bodies, and later in the terminals. In some species pretreatment with monoamine oxidase inhibitors, such as Nialamide tends to increase fluorescence, while pretreatment with norepinephrine and dopamine increase fluorescence all along the neuron (Falck, 1962; Norberg and Hamberger, 1964; Fuxe and Sedvall, 1965). Sectioning or com- 
pressing a peripheral sympathetic nerve causes an increase in fluorescence on the central side of the obstruction (Norberg and Hamberger, 1964), and results in an accumulation of electronmicroscopy discernable granules at this location (Kapeller and Mayer, $1966 \mathrm{a}, \mathrm{b}$,$) . In some of the fluorescence experiments$ the borohydride reduction test of Corrodi et al (1964) was used to differentiate catecholamines from non-neuronal fluorescent structures such as elastin.

B. Connective Tissue of the Arterial Wall. 1. Elastin. a. Content in the Arterial Wall. The arterial wall contains a considerable amount of elastin.

Lowry et al(1941) extracted everything in the wall except elastin by boiling arterial samples in dilute alkali. The remaining elastin represented between 24 and $30 \%$ of dry tissue weight of the human and rat aortae.

Hass (1942) extracted elastin from human aortae with formic acid. The arterial wall was found to contain between 28.9 to $42.2 \%$ elastic tissue with no apparant relation to age.

Meyers and Lang (1946) used the method of Lowry et al (1941) to determine that $37 \%$ of the dry weight of young aortic media and intima was elastin. With age this value decreased to about $26 \%$.

Neuman and Logan (1950) determined the elastin con- 
tent of whole bovine, $p 19$, and rat aorta to be $39 \%, 57 \%$, and $47 \%$ respectively. The method used was to remove most of the non-elastic tissue by boiling in water. The remaining elastin was assessed by a colorimetric determination of hydroxyproline by weight. This value is in agreement with amino acid analyses of elastin derived from various tissues

Faber and Moller-Hou (1952) used the methods of Lowry et al (1941), to analyze for elastin in the media and intima of normal and hypertensive human aortae. In normal aortae elastin ranged from $35 \%$ of dry tissue weight at age 20 to $20 \%$ at age, 80 .

Kraemer and Miller (1953) determined by removal of all but the elastin that the presence of atheromatous plaques was not correlated with alterations in the elastin content of the aorta. Both normal and atheromatous human aortae contained about $35 \%$ elastin by dry weight.

Harkness et al (1957) surveyed the elastin content of different portions of the arterial tree from the aorta through the saphenous artery. The tissue was autoclaved to remove collagen, and then boiled in $0.1 \mathrm{~N}$ alkali to remove noncollagenous, alkali-soluble proteins. The systemic arterial tree was found to be divided into two distinct regions. In the thoracic aorta from the aortic valve to the diaphragm, the ratio of elastin to elastin-plus-collagen by dry weight was 
50 to $60 \%$. Distal to the diaphragm the ratio of elastin to elastin-plus-collagen dropped to 25 to 30\%. The entire transition was found to occur over a $5 \mathrm{~cm}$ length of the aorta. Scarselli(1961) stained the elastin of human aortic tissue with orcein dye, and then enzymically hydrolyzed the tissue. Photometric analysis gave quantitative estimations of olastin content. Elastin was found to represent about $20 \%$ of the wall at a few months of age, rising to nearly $50 \%$ at age 20.

Hosada and Minoshima(1965) assessed the elastin content of human aortae and pulmonary arteries using Scarselli's orcein dye method. Both the systemic and pulmonary aortae contained about 30 to $35 \%$ of dry weight taken from subjects up to 10 years of age. This percentage gradually fell with each decade to about $22 \%$ at the seventh decade of $11 \mathrm{fe}$.

Smith et al (1957) demonstrated by differential staining, that reticular or collagenous fibers are often seen closely applied to the elastic lamellae in the media in the aged mouse aorta. Increased quantities of ground substance were also observed. It appears that the decrease in elastin from about 35 to about $25 \%$ seen with aging reported by Meyers and Lang (1946), Harkness et al (1957) and by Hosada and Minoshima (1965) may result, in part, from an increase in the absolute quantity of other connective tissue elements, mainly collagon. 
Fischer and Llaurado (1966) reported that the elastin content of systemic arteries varies with different vessels. Most medium-sized arteries, carotid, femoral, and mesenteric, contained 20 and $35 \%$ elastin by dry weight. The aorta contained $40 \%$ elastin by weight, while renal and coronary arteries contained only 15 to $18 \%$ elastin by dry weight. In other experiments these authors (1967) found no difference between "the elastin content of these in normal dogs as compared with those obtained from renal hypertensive dogs. These studies showed, as did those of Harkness et al (1957), that the elastin content of the pulmonary artery resembles the peripheral systemic arteries (about $26 \%$ elastin), rather than the thoracic aorta (about $40 \%$ elastin).

Apter et al (1966), using histological and chemical analyses, also reported on the elastin content of the aorta. The ascending aorta contained $45 \%$ of dry weight. This value declined with increasing distance from the aortic valve.

The reason for the differences in distribution of elastin in arteries is not clear; the sharply defined increase in the thoracic aorta is especially baffling. The high content of elastin falls to correlate consistently with transmural pressure, negative extravascular pressure, or the intrathoracic movements associated with respiration. Neither the pulmonary artery, nor the systemic arteries of hypertensive 
animals exhibits the high elastin contents of the thoracic aorta, while even the thoracic aorta of newborn puppies show these high contents.

b. Morphological Appearance. The morphological appearance of elastic tissue depends upon the tissue from which it is derived. In ligamentum nuchae it is densely-packed with the appearance of parallel fibers. In the arterial wall the elastic fibers are condensed into flat sheets forming curved, fenestrated lamellae which lie concentric to the lumen. Under the light microscope the elastic lamina appear to be composed of two or more kinds of elements (Dees, 1923). The first is a coarse network in which the thick fibers show branchings. These fibers are 3 p or less. The second consists of very fine fibrils. More recent studies, however, suggest that these size differences may not reflect any basic differences in fiber proportion.

The earliest electronmicroscopic studies of elastin were performed by Wolpers(1944) on pepsin-treated ligamentum nuchae and aortae. Fibers ranging from 200 to $2500 \AA$ diameter were observed. Studies on elastin obtained from ligamentum nuchae (Gross, 1949; Lansing et al, 1952), carp swimbladder (Gross, 1949), aorta (Lansing et al, 1952), skin (Bahr, 1951, 1955; Linden ot al, 1955) and alveoli (Laitinen, 1960) all 
revealed fine intertwined fibrils. On the other hand, other studies on carp swimbladder (Franchini and deRobertis, 1951) and skin (Tunbridge et al, 1951) failed to indicate a fibriller structure. Ultrathin sections of ligamentum nuchae, 500 to $1000 \AA$ thick (Dettmer, 1956; Usuku, 1958) revealed elastin to be a reticular network of beaded fibrils, 100-200 $\AA$ in diameter, with no axial periodicity.

In 1949, Balo and Banga isolated an elastolytic enzyme (elastase) from the pancrease. This enzyme was distinct from purified trypsin or chymotrypsin (Balo and Banga, 1950). Treatment of ligamentum nuchae with elastase was found to release intertwined fibers, $2 \mu$ in diameter (Lansing ot al, 1952) or, if treated for long periods of time, to convert the insoluble fibrous material to a soluble, globular form (Balo and Banga, 1950). Enzymatic hydrolysis of collagen-free aortic media (Hall et al, 1955) also provided fibers after 30 minutes treatment. After 90 minutes treatment, $200 \AA$ fibrillar units were seen; after 20 hours treatment, nothing structurally distinct remained. Ultrathin sections of ligamentum nuchae subjected to elastase treatment (Yokata, 1957) revealed that the first signs of degradation were the appearance of pit-like depressions on the outer edge of the fibrils. With longer olastase treatment the fibers split longitudinally into fine fibrlls composed of beaded chains. 
The electronmicroscopists gradually separated into two camps on the issue of homogeneity of elastin. One group described elastic tissue as a homogenous system composed entirely of anastomosing, rope-like fibrils. Studies on lung (Woodside and Dalton, 1958), bronchiolar tunica propria (Karrer, 1958), aorta (Ayer et al, 1958; Cox and Little, 1961; Jensen, 1962) and ligamentum flavum epiglottis, pinnae, vocal chords and renal arteries (Cox and Little, 1961) all supported this point of view. When thin films were stripped from arter-. lal elastic lamina (Ayer and Eisenstein, 1963) even swelling. with dilute formic acid failed to indicate any discernible multiphasic structure. A second group of electronmicroscopists.subscribed to the view that elastin is a multi-component system, composed of fibrils embedded in an underlying matrix. Studies on ligamentum nuchae (Gross, 1949; Lansing et al, 1952; Tunbridge et al, 1952; Creenlee et al, 1966); carp swim bladder (Gross, 1949), and tracheal mucosa tunica propria (Rhodin and Dahlman, 1955) all provided evidence for a heterogeneous structure of elastin. X-ray diffraction studles indicated, in agreement with the electronmicroscopic studi, les, that elastin is a disordered, non-crystalline, fibrous substance, in both the stretched and retracted states (Kolpak, 1935; Astbury and Bell, 1939; Astbury, 1940; Cox and Little, 1961). Elastin thus appears to be a fibrillar structure 
composed of bead-like units. The fibrils seem to be interconnected at many points, producing a loosely-organized, random-like structure, which exhibits great extensibility in all directions.

\section{Chemistry. Elastin is a protein. Amino acid} analyses (Neuman, 1949; Bowes and Kenten, 1949; Partridge and Davis, 1955; Smith et al, 1963) indicate that elastin is about $25 \%$ glycine, $20 \%$ alanine, $15 \%$ valine, $9 \%$ leucine, and $1.5 \%$ hydroxyproline. Small amounts of most other amino acids are also present except for those containing sulfur.

Apout $2 \%$ of the dry weight of elastin is mucopolysaccharide, but it has not been possible to remove the mucopolysaccharide except by hydrolysis with the elastomucase component of elastase (Hall et al, 1952; Hall, 1953; Loeven, 1960), or by repeated autoclaving (Cotte et al, 1963). There is also evidence that elastin contains small amounts of lipid. A weak osmophilia and light blue-black staining reaction with Sudan black have been reported by Lansing et al (1952), and lipid corresponding to $0.5 \%$ of the dry weight of elastin has been recovered following elastase degradation of chemically extracted elastin (Lansing et al, 1952) and defatted elastin (Labella, 1957). Banga and Schuler (1953) obtained up to 12\% lipid by elastase degradation. The difficulty in separating mucopolysaccharides and lipids suggests that they are either 
intrafibrillar or very tightly bound to the surface of the elastin fibers. The small amounts of these compounds suggest that they probably do not act as a matrix embedding the fibrous elements and do not play an essential role in the structural integrity of elastin. Ayer ot al (1958) used formic acid to swell and split aortic elastin into fine, electronmicroscopically distinct fibrils. When the formic acid was neutralized the swollen fibrils shrank and reaggregated to their original appearance. Wood (1954) observed that agents which disrupt the integrity of mucopolysaccharides have negligible effects upon the tensile strength and load-extension characteristics of natural and purified ligamentum nuchae. However, Banga and Balo (1961) demonstrated that the tensile strength of human aortic strips is markedly lowered by pancreatic extracts. Heterogeneous elastase slightly lowered the tensile strength at $90 \%$ extension, but the elastomucase fraction of the enzyme lowered it to one-half of control at approximately the same extension. It is on this basis that Banga and Balo (1961) classify elastin as a lipoprotein.

The chemical structure of elastin has been extensively studied by Partridge and his associates. Partridge and Davis(1955) dissolved elastin in boiling oxalic acid. An $\propto$ fraction (MW 60,000-80,000) and a $\beta$ fraction ( $M W 5,500-6,000$ ) were obtained. The amino acid composition of these fractions 
and of whole fibers were found to be very similar, indicating a fairly homogeneous structure. Terminal analyses on the $\alpha$ and $\beta$ fractions indicated that the fibers are probably composed of two chains. Stepwise enzymatic degradation of elastin (Partridge et al, 1963) yielded the usual amino acids and a fluorescent peptide which contained up to $30 \%$ of two unknown amino acids. Complex fractionation procedures (Thomas et al, 1963) isolated these amino acids and permitted their chemical characterization. The fluorescent amino acids were determined to be a substituted quarternary pyridinium compound. The compound was called desmosine, and its isomer, isodesmosine. Information concerning the biosynthesis of desmosines has been obtained from the rupture-prone arteries of copper-deficient chickens (O'Dell et al, 1961) and pigs (Weissman et al, 1963). Elastin obtained from these arteries swells to an abnormal extent in water and is readily soluble in alkali. The amino acid composition of this elastin exhibits up to three times the normal lysine content (Starcher et al, 1964). Partridge and coworkers $(1964,1966)$ extended these observations by injecting normal rats with $C_{14}$ - labelled lysine. Within 11 days, $80 \%$ of the label was incorporated into the desmosines, and all of the remaining label was found only in lysine. Similar results were obtained when labelled lysine was introduced into tissue cultures of aortic slices (Miller et al, 1964). However, 
labelled lysine was not incorporated into the desmosines in experimental lathyrism, induced by the administration of $\beta$-amino proprionitrile (D'Dell et al, 1966). This agent is known to induce malformations of the elastic lamina, and to decrease the arterial content of elastin (McCallum, 1965a,b). During the course of these studies, Partridge evolved the viow that fibrous elastin may be formed by the cross-linking of a soluble elastin precursor at the site of fiber growth, probably under the influence of a copper-containing enzyme. The H-shape of the desmosines, the large size resulting from their configuration, and the decreased tensile strength of arteries in which desmosine synthesis is impaired all suggested that the desmosines may function as bridges, cross-linking parallel amino acid chains in elastin. Such a linkage would require the correct apposition of four lysine links on at least two independent peptide chains. This would give rise to functional units or "corpuscles," analagous to the beads described by Yokata (1957). The theoretical enclosed volume of such a corpuscular model was calculated to be $66 \%$ of total molecular volume, a value which compares favorably with the experimentally-determined $63.1 \%$ water volume of hydrated bovine ligamentum nuchae (Partridge, 1967). The importance of cross-links will be considered in the following discussion of the mechanical properties of elastin. 
d. Mechanical Properties. 1. Thermoelastic behav10r. The most striking property of elastin is its characteristic reversible, long-range deformablity. This is especially important in the case of the elastic arteries where recoll of the wall plays an important role in maintaining an effective perfusion pressure during diastole. The property of rubberlike elasticity is rare, and is exhibited by very fow substances. These are usually termed elastomers. The properties of elastomers have been summarized by Baumann (1959):

a) They exhibit rapid and long-range extensibility, elongating to strains of 1.0 or more.

b) They exhibit high elastic moduli and tensile strength at maximum strains.

c) They retract rapidly when released, often exhibiting rebound.

d) Upon release of an elongating stress, they reiturn to their original dimensions. According to thermoelastic theory, typical elastomers behave as though they were composed of long, fairly independent polymer chains. Flexible bonds within the chains, and only occasional cross-links between the chains permit rapid and extensive elongation under stress. Under resting conditions the polymer chains, lie in a shortened, crumpled state possessing maximum entropy. When such a system Is subjected to a stress it extends with straightening of the 
chains; upon removal of the stress the chains readily retract, resulting in a reversible elasticity. Heating elastomers increases the kinetic energy of the chains. This increases the entropy and results in a further shortening of the chains (Gough-Joule effect). Rubber, the paragon of elastomeric behavior, actually does shorten when it is heated. Rubber differs from proteins in that rubber is formed by long hydrocarbon chains with few interchain bonds, while proteins usually possess many radicals capable of formimg weak interchain bonds. In order to exhibit elastomeric behavior, therefore, the radicals in the protein structure must be neutralized by a solvent to reduce interchain "friction." Rubber 18 extensible? even when dry, while proteins are inextensible and brittle unless a solvent is present. Rubber also cryetallizes upon stretching (Guth, 1947) and this undoubtedly underlies much of the extension-retraction hysteresis seen with rubber. McCartney (1913) freed bovine ligamentum nuchae of much of its collagen content by pepsin digestion, and subjected strips to increased temperature. The ligament was found to contract linearly with increased temperature, elongating along the same line upon cooling. It is probable that the preparation actually contained considerable amounts of collagen because the strips were found to be perfectly reversible only up to $65^{\circ}$, the temperature at which native collagen exhibits thermal retraction 
(Gustavson, 1956; Piez and Gross, 1960). Meyer and Ferri (1937) and wohlisch et al (1943) showed that untreated ligamentum nuchae, when $100 \%$ extended, conformed to elastomeric theory by contracting when heated. Because of an apparant increase in internal energy when extended, Meyer and ferri concluded that crystallization took place at large extensions. However, Hoeve and Florey (1958) also studied unpurified ligamentum nuchae, but did so in a 30\% glycol-water mixture. This mixture was used because in this solvent the volume of the tissue is constant over a wide range of temperatures, thus avoiding complicating changes in cross-sectional area. Their data also supported the view that elastin is a typical elastomer, but calculation of internal energy indicated that crystallization does not occur in the stretched state. This is in agreement with $X$-ray diffraction data of stretched elastin (Astbury, 1940).

The behavior of aortic elastin is also predicted by (elastomeric theory. Lloyd et al (1948) studied strips of ox aortic elastin after removal of collagen with formic acid. Load extension curves were obtained in water. For a given extension the load was $60 \%$ higher at $75^{\circ} \mathrm{C}$ than at $15^{\circ} \mathrm{C}$, indicating - greater resistance to.distention at the higher temperature. 2. Mechanical elasticity. Many studies have been reported describing the mechanical properties of elastin 
preparations. Ligamentum nuchae has often been used to represent the mechanical properties of elastic tissue. Reuterwall (1921) also studied the elasticity of bovine ligamentum nuchae. He found this tissue was extensible up to about $20 \%$ of resting length. His data have been converted by Burton (1954) to conventional stress-strain terms. According to these measurements the elastic modulus of ligamentum nuchae is about $4.0 \times 10^{7}$ dynes $/ \mathrm{cm}^{2}$. This value is higher than most other reports of elastic tissue, and may reflect the $9 \%$ collagen present in unpurified ligamentum nuchae. Krafka (1939) also studied the length-forçe relationships for strips of ox ligamentum nuchae. He found the elastic modulus to be about $3.0 \times 10^{6} \mathrm{dynes} / \mathrm{cm}^{2}$, and virtually linear throughout the entire extension range. Remington (1957) did not find the elasticity of ligamentum to be linear, but rather that it progressed from $1.5 \times 10^{6}$ dynes $/ \mathrm{cm}^{2}$ at moderate extensions, to $4.1 \times 10^{6}$ dynes $/ \mathrm{cm}^{2}$ at large extensions. Again, the presence of small amounts of collagen may have influenced the elasticity at large extensions. Hass(1942) extracted elastin from human aortae with $88 \%$ formic acid. The thermal-retraction of this elastic tissue was found to be greater than that for the intact blood vessel. Burton (1954) recalculated Hass' data and determined the elastic modulus to be about $3.0 \times 10^{6} \mathrm{dynes} / \mathrm{cm}^{2}$. The tensile strength of aortic elastin was found to be between 
1.5 to $6.7 \times 10^{6} \mathrm{dynes} / \mathrm{cm}^{2}$. The fact that the tensile strength bore no relationships to the amount of elastic tissue in multiple preparations suggests the presence of a stiff parallel element which sustains the load at large extensions, and determines the tensile strength. Presumably this parallel element is collagen.

Wood (1954) obtained load-extension diagrams of ligamentum nuchae following a number of different treatments. After treatment with pancreatic elastase the preparation exhibited collagen-like behavior. Interestingly, however, the elastase-digested ligament had to be stretched to about $70 \%$ of resting length before showing collagen-like elasticity. It was irreversibly extensible up to this length. Once stretched to this length, however, the preparation exhibited the highly elastic behavior of collagen. This suggests that at normal lengths the collagen is probably slack, and offers little resistance to extension. In other experiments ligain mentum nuchae was boiled in $1 \%$ acetic acid to dissolve collagen. Hyaluronidase and calcium chloride dissolve, and may also degrade, mucopolysaccharides. Treatment of the collagen-free ligament with these reagents resulted in almost no change in the elastic properties. These data suggest that mucopolysaccharides associated with astin contribute little to the mechanical properties. 
Ayer et al (1958) reported that the maximum extension of formic acid extracted aortic rings was $127 \%$, as compared with $87 \%$ for the intact aorta. Extension curves for extracted rings rose steeply, then flattened out as the specimen resisted further distentions. Following an $80 \%$ extension of aortic rings, recoil was found to be incomplete unless unloaded to less than initial load. However, after treatment with formic acid to remove all but the elastin, recoil following extension was found to be perfect, although it required long durations for completion. Static hysteresis was present in untreated aortic rings, and was observed to increase with formic acid extraction.

In 1961, Hall and Czerkawski studied the elastic modulus of ligamentum nuchas as an indication of the enzymatic degradation produced by elastase. Preparations of ligament were treated to remove collagen and cut into strips. Young's modulus of elasticity increased linearly with length, up to $30 \%$ extension. Extrapolation of their curves to zero time of enzymatic incubation indicates a modulus of $1.1 \times 10^{7} \mathrm{dynes} / \mathrm{cm}^{2}$ in the natural state. These preparations were, however, partlally dehydrated, so that their data were determined on the basis of a decreased cross-sectional area, and may not truly roplect the normal, in situ properties of elastin.

Two experiments have also been undertaken on "single" 
elastic fibers. Redenz(1926) teased single elastic fibers free from bovine ligamentum nuchae. He observed that even with single fibers, 130 - $140 \%$ extensions could be produced before breaking. Carton et al (1962) studied single fibers from ligamentum nuchae more quantitatively. Cross-sectional areas were not determined so moduli of elasticity were not avallable. However, load-extension curves were obtained. These curves indicated that strain was an exponential of the applied load. Maximum strains were found to be about $130 \%$, although considerable variability was seen. The most unexpected observation was that single fibers were actually less extensible than the parent strip from which they were obtained. Staining with Masson's trichrome revealed only negligible amounts of collagen relative to the total size of single fibers, while staining of the parent strips indicated that they contained considerable amounts of collagen. The presence of collagen apparently did not impair the ability of the parent fibers to stretch, since strips were found to be more extensible than single fibers. These observations agree with the findings of Wood (1954) who compared untreated ligamentum nuchae preparations with collagenfree strips of ligament. The difficulty in assessing studies on single fibers is the evaluation of the term "single", if Indeed a minimum material may ever be obtained which still exhibits mechanical behavior that is representative of elastin. 
If these single fibers were truly devoid of collagen, then the finding that the isolated fiber is less extensible than the parent strip is most interesting. It may be that the reason for the difference lies in the state of the elastin before extension, i.e. that the single elastin fiber is actually more fully retracted than the parent strips when each is in 1ts respective unextended state. It seems either thats 1) the parent strip may actually be composed of a random network of single elastin fibers, and these are extended into a parallel orientation with extension, something like that which occurs with the pulling on the corners of a handkerchlef; or 2) that the elastic fibers in the unextended parent strip somehow are partly elongated by parallel collagenous fibers. It is also possible that the increase in maximum extensibility of the single fibers might have resulted from chemical damage to the elastin during the purification procedures.

2. Collagen. a. Collagen Content of the Arterial Wall. The arterial wall contains significant quantitaties of collagen. Two methods have been employed to determine content. In both methods the arteries are dehydrated and collagen is expressed in terms of percent of dry tissue weight. Lowry et al (1941) reported a method for determining collagen content. They chemically removed all substances from the tissue except collagen and elastin. Boiling this tissue in dilute alkali 
removed the collagen. The difference between the collagen-pluselastin and the remaining elastin was used as a measure of collagen content. Using this method, Lowry et al (1941) found that the collagen content of the aortae of both man and rat amounts to 28 to $31 \%$ by dry weight. Myers and Lang (1946) used the same methods on the thoracic aortae of humans of various ages. The collagen content varied from 12 to $24 \%$ increasing with age. Faber and Moller-Hou (1952) also used these methods to determine the collagen content of adventitia-free human aortae at different ages. Normal aortae contained $20 \%$ collagen at age 20 , and this value rose to $35 \%$ at age 80 . Hypertensive aortae showed more collagen and less elastin at each age than normal aortae. Particularly interesting was the fact that the increase in collagen exactly accounted for the decrease in elastin, providing circumstantial support for the hypothesis of Burton et al (1955) that collagen and elastin may be interconverted.

A secend method of collagen determination was devised by Neuman and Logan (1950). It was based on the fact that collagen is one of the few animal proteins which contains appreciable amounts $(13 \%)$ of hydroxyproline. Use of a colorimetric hydroxyproline method indicated that the collagen content of rat and beef aorta was about $24 \%$ by dry weight. Pig aorta.was found to be only $16 \%$ collagen. Harkness et al (1957) 
used hydroxyproline analysis to determine collagen content of many large arteries in the dog. Collagen was found to be about three times as plentiful as elastin in most arteries. In the thoracic aorta it was present in amounts equal to elastin. Ebel and Fountaine (1964) used the method of hydroxyproline analysis to determine collagen content of bovine aortae at various ages. The collagen content of aortae at age 2 was $33 \%$ of dry weight, while the aortae of older animals contained 40\%. Apter et al (1966) have used both histological and hydroxyproline analyses in determining the collagen content of dog aortae. About $15 \%$ of dry weight was collagen in all regions of the thoracic aorta. This value rose to $22 \%$ in the abdominal aorta. These data agree qualitatively with the findings of Harkness ot al (1957). Finally, Fischer and Llaurado (1966) found slightly higher values for collagen content of various systemic arteries. Hydroxyproline analyses were also used. The ascending aorta was found to contain only $19.6 \%$ collagen. Abdominal aorta, mesenteric, coronary, renal, femoral, and carotid arteries contained 36 to $50 \%$ collagen. In other studies, the same authors (1967) rendered dogs hypertensive by wrapping one kidney for periods up to six weeks. Arteries obtained from these hypertensive dogs exhibited about $10 \%$ less collagen than vessels obtained from normal control animals. It is clear from the above studies that the collagen 
content of arteries is about 20 to $40 \%$, increasing with age. It is also evident that, even in the same animal, the collagen. content varies from one artery to the next; it is lowest in the thoracic aorta, and tends to be higher as one proceeds toward the periphery. Collagen forms a significant percentage of the arterial wall, and may influence its mechanical properties. If the collagen fibrils were in an extended state under normal conditions they would impart great stiffness; if they were in a slack state their potential inextensibility would not be manifest. Morphological studies by Wolinsky and Glagov (1962) indicate that the appearance of medial collagen changes from wavy lines at low pressure to smooth straight lines when arteries are distended with pressures of up to $200 \mathrm{~mm} \mathrm{Hg}$. Adventitial collagen, however, remains somewhat wavy even at these high pressures.

\section{b. Morphological Appearance. Light microscope}

studies of collagen indicated that collagen was composed of bundles of fibers. Electronmicroscopic studies showed that these fibers are composed of finer fibrils. Early electronmicroscopic studies of collagen were reported by Schmitt et al (1942) using unstained material derived from skin. Their preparations showed that collagen exhibits axial bands with a 640 A period. These bands consisted of a succession of dense (A) and light $(B)$ bands in the axial direction. The light bands 
were about four times the length of the dark bands, and appeared to be more extensible because when exterided the relative proportion of the period occupied by the $B$ band increased more than the $A$ band.

Wolpers (1948) used osmic acid as a fixative and a stain. In these preparations two dense bands and one light band per period were found. Six intraperiod bands were observed by Nutting and Borasky (1948) and by Schmitt and Gross (1948) using phosphotungstic acid stain and platinum and chromium shadowing. These bands do not appear to be artifactual because ultrathin sections of collagen fibrils from the lamina propria of the frog eye show the same six bands within the interband, and these appear uniformly across several fibrils (Rhodin and Dalham, 1955; Wasserman er al, 1957). Unlike elastin, the electronmicroscope observations on collagen have been largely uncontroversial, and repeatedly substantiated.

Wide angle $X$-ray diffraction studies have also been performed on collagen fibrils. Astbury (1940) observed an appreciable sharpening of the pattern when the fiber was stretched, but did not notice any change in the spacings. Ramachandran and Kartha (1955) interpreted this sharpening of the pattern as an indication that the individual fibrils are not aligned parallel to one another in the retracted state, 
but describe an angle with the fiber axis. Stretching was then thought to bring the fibrils parallel.

Low angle $X$-ray diffraction studies were performed by Bear (1942) who obtained a large number of reflections, all of which were higher orders of a fundamental spacing of $640 \AA$. ic These values agree with the observations of the electronmicrot scopists. In dry fibers the long spacing increases in proportion to mechanical extension, up to about $10 \%$, at which length a dry fiber breaks. Upon hydration the long spacing also increases (Boulduan and Bear, 1950, 1951; Cowan et al, : $(1955)$.

c. Chemistry. The chemical composition of collagen has been studied extensively. Bowes and Kenten (1949) and Eastoe (1955) have published tables summarizing the amino acid composition. About one-third of the total number of amino acid residues are glycine. Proline and hydroxyproline together form about two-ninths of the total, while alanine forms approximately one-ninth. The content of the polar residues Is about two-ninths, while the remainder is composed of neuHtral residues. The model of collagen structure which is most generally accepted is that of Ramachandran and Kartha (1954, 1955) deduced from wide angle $X$-ray diffraction studies. This model consists of three hellces running beside one another and stabilized by interchain hydrogen bonds between NH and $C O$. 
The stability of the intact molecule is believed to derive from the interchain hydrogen bonds. Breakage of these hydrogen bonds is believed to occur with heating, resulting in thermal contraction. Repeating of the structure after every 216 residues has been calculated to form a $618 \AA$ period, essentially agreeing with the $640 \AA$ banding observed in electronmicroscope and $X$-ray diffraction studies. The helices form a triple helix, i.e. they are coiled, not only around one another, but all three chains are also coiled around a central axis. A spiral structure is supported by hollow, tubular structures seen in electronmicrophotographs of cross-sections of collagen (Kennedy, 1955). According to Banga (1966), the presence of double bonds along two-thirds the length of the collagen chain is the source of restricted mobility. These double bonds, coupled with the presence of additional interchain hydrogen bonds, are probably the reason for the rigid, inextensibility of collagen.

d. Mechanical Properties. 1. Thermoelastic bohavior. A characteristic property of collagen is that, upon heating $\left(60-65^{\circ} \mathrm{C}\right.$ for native collagen-Gustavson, 1956; Piez and Gross, 1960) the fiber contracts to one-third its original length. This property differs from the thermal contraction of lostin, in that with collagen it is: Irreversible. The typical $x$-ray patterns disappear following thermal shrinkage, and are 
not restored by cooling (Bear, 1942). Following heating, the banded appearance is lost and the shrunken fibers are susceptible to chemical and enzymatic proteolysis (Banga, 1966). An interesting observation (Verzar, 1956), is that the load necessary to prevent thermal contraction increases with age. 2. Mechanical Elasticity. The elastic modulus of collagenous tendons has been determined. Reuterwall (1921) reported load-extension data for various human muscle tendons. Burton (1954) used these data to determine that the elastic modulus of tendons is about $1 \times 10^{9}$ dynes $/ \mathrm{cm}^{2}$. Krafka (1939) reported the incremental elastic modulus of collagenous tendons to be $3.0 \times 10^{7}$ dynes/cm². Verzar (1956) reported load-extension data for collagenous rat tail tendon with a cross-sectional area of $.15 \mathrm{~mm}^{2}$. Because the extensions are very small, one may assume a constant cross-sectional area, and then convert his load-extension data into elastic moduli. At $2 \%$ extension the incremental modulus equals $2.9 \times 10^{9}$ dynes $/ \mathrm{cm}^{2}$; at $4 \%$ extension it equals $5.8 . \times 10^{9}$ dynes $/ \mathrm{cm}^{2}$. A stress-strain fcurve of collagenous tendons published by Remington (1957) Hindicates an elastic modulus of $8.3 \times 10^{7} \mathrm{dynes} / \mathrm{cm}^{2}$. Mustacchi (1951) loaded thin strips teased from rabbit tendon. These were loaded with weights, frozen with dry ice, and preipared for electronmicroscopic study while under constant load. The axial period was observed to increase greatly, in a fow cases to as much as 7000 . In other cases, flbers were ob- 
served to have ruptured. The load in these experiments was unspecified, and the histological preparation perhaps harsh; the observation of ruptured fibers attests to the possibility that extension beyond the yield point had been induced. One.... must be skeptical of the meaning of these experiments without proof of reversibility, because extensions of 1000 times the axial period far exceed the few percent observed in all of the whole-tissue studies.

Skin is composed of a network of collagenous fibers. Conabere and Hall (1946) determined the Young's modulus in dry, tanned, leather fibers. Nalues of $1 \times 10^{10} \mathrm{dynes} / \mathrm{cm}^{2}$ were observed over the linear portions of the stress-strain curves. The fibers also showed some irreversible extension following the first loading. Hall (1951a) loaded wet fibers from ox skin for long periods of time. The rate of extension occurred rapidly at first, and then proceeded slowly with additional time. Morgan (1960) studied raw and tanned skin collagen fibers. Various tanning processes altered the loadextension curves, usuelly increasing the elasticity over untreated fibers. Untanned fibers gave almost linear loadextension curves, gradually increasing in stiffness with large extensions. The structure of skin differs from that of tendon. In skin a loose, network-like arrangement of flbers is found, tos compared with tendons, in which densely-packed collagen 
fibers are found. This may account for some of the alinearity of skin. Hall (1951b) obtained stress-strain curves on similar preparations obtained from ox skin, and also recorded the cross-sectional area. The elastic modulus was found to vary, without regard to extension, between $7 \times 10^{9}$ and $1.8 \times 10^{10}$ dynes/ $\mathrm{cm}^{2}$. Calculation of the work of extension was found to be virtually constant over a pH range of 4 to 11. Recently the Glasgow biomedical engineering group (Gibson et al, 1965; Kenedi et al, 1965) has"reported mechanical studies on skin. Their stress-strain curves show a gradually rising slope at low strains, and steep, virtually linear slope at higher strains. Histological analyses of the preparations fixed at various degrees of strain indicated that the gradually increasing portion of the stress-strain curves correlated with an increasingly parallel alignment of fibers in the collagenous network. At very low strains these fibers were random in their orientation. Elastic fibers are also present, and appear to be interconnected with the collagen fibers. Calculation of an incremental elastic modulus from the slope of the linear portion of their stressstrain curves gives a value of approximately $2.9 \times 10^{8}$ dynes/ $\mathrm{cm}^{2}$. This value is undoubtedly influenced by the presence of water, elastin and other non-collagenous materials. Contraction-deformation curves show hysteresis with extension 
exhibiting greater loads than retraction at each strain. An assymetric network model was developed incorporating a recruitment phenomenon to account for the alinearity of the stress-strain curves.

Cartilage is composed of a well-ordered combination of collagen fibers and ground substance. Hirsch (1944) presented the load compression data on rib cartilage of Rauber and Triepel who found elasticity values of .8 to $1.5 \times 10^{8}$ dynes/. $\mathrm{cm}^{2}$. Hirsch's (1944) studies on femoral condyle failed to provide original, unstressed dimensions, and therefore did not permit calculation of elastic properties in exact terms. However, Hirsch (1944) did note that an index of elasticity increased with increasing strains. He also observed that the preparations exhibited delayed recovery of strains when the stress was removed. This delay persisted even after 30 minutes. Although cartilage is not purely collagen, it does reflect the elasticity of collagen, and even more important, it reflects the properties of two constituents of the arterial wall.

The tensile strength of collagen is very high. Bader (1963) presented the data of Wohlisch, du Mesmil de Rochemont, and Gerschler who studied tendon and ligamentum nuchae. These authors found the tensile strngth of tendon to be $6.6 \times 10^{8}$ dynes $/ \mathrm{cm}^{2}$. Although this value is low compared with other reported values for the elastic modulus of tendon, it is about 
25 times as high as the same authors reported for elastin. Mitton and Morgan (1960) found the breaking load of vegetabletanned leather fibers was $1-5 \times 10^{9}$ dynes $/ \mathrm{cm}^{2}$. A breaking stress of $5 \times 10^{9} \mathrm{dynes} / \mathrm{cm}^{2}$ was also reported by Harkness (1961). The resistance of collagen to fracture following Plexion has also been investigated. Mitton and Morgan (1960) found the breaking load of vegetable-tanned collagen flbers was reduced after repeated flexion. Maeser (1944) observed that when. leather is flexed it becomes more plastic, and tends to crack along the line of flex. Cater and Kanagy (1954) also subjected leather to a flex test by sliding it over rollers arranged to produce flexion of between $90^{\circ}$ and $180^{\circ}$. A nearly straight-line relationship between axial load, and the log of time before cracking was found. Increased temperature decreased the time before cracking occurred. This suggested to the authors that flexure-degradation was probably the result of flowing, and ultimately fracture, of the individual fibers. Fatigue due to flexure is probably not a serious consideration in the consistently cylindrical arterial wall.

The mechanical properties of collagen- the high elastic modulus and the high tensile strength- play an important role in maintaining the integrity of the arterial wall. The histological observations of Bader and Kappel (1958) and Wolinşky and Glagov (1964) indicate that a significant percent.. 
of the arterial collagen is slack at moderate vessel distentjons; at $100 \%$ distention or more, medial collagen is almost entirely extended. Studies on human iliac arteries before and after trypsin hydrolysis of elastin (Roach and Burton, 1957) showed that enzymic removal of elastin shifts the tensionstrain curve to the left. The final slope of the tensionstrain curve of the untreated vessel was virtually identical with that of the vessel composed predominantly of collagen. This is strong evidence for the parallel arrangement of collagen and elastin with one another. If collagen were in series with other elements within the wall, then destruction of any of these elements would undermine the integrity of the wall.

3. Ground Substance. a. Content of the arterial wall. Several studies have been reported concerning the arterial ground substance. Analyses indicate that bovine (Berenson, 1959) and human (Gore and Larkey, 1960) aortic ground substance is largely mucopolysaccharide. This represents $1.3 \%$ of the dry tissue weight; in sclerotic arteries it represents $2.5 \%$ (Buddeke, 1960). Bertelsen and Jensen (1960) examined aortae from human subjects of neonatal age through age 90. The ground substance contained both hyaluronic acid and sulfated mucopolysaccharides. Fetal and newborn aortae contained hyaluronic acid as the major component. With aging, the sulfated mucopolysaccharide content of the intiria and media increased 
until age 50. Similar findings were reported by Kaplan and Meyer (1960).

b. Mechanical Properties. Studies on the mechanical properties of synovial fluid (Chain and Duthie, 1939; Davies, 1944), and its major component, hyaluronic acid (Balazs and Laurent, 1951) revealed that these substances exhibit viscosities that are 5 to 300 times that of water, increasing with hyaluronic acid concentration. Although studies on the mechanical properties of arteries following hyaluronidase treatment have not been reported, it seems probable that the viscosity th

Fof the ground substance may influence the time-dependent behavior of the arterial wall. In retracted vessels the elastic lamina are markedly corrugated. The transition between the corrugated and the straightened condition requires the ground substance to flow around, and perhaps through the fenestrations in the elastic lamina. Moreover, at small radi large changes in dimension occur per unit change in pressure. It seems likely, therefore, that the viscosity contributed to the wall by the ground substance would be most evident at small dimensions. The energy required to overcome this viscous resistance may also contribute to pressure-diameter or loadlength hysteresis.

C. Cellular Components of the Arterial Wall.

1. Endothelium. Circumstantial evidence suggests that 
the endothelial cells secrete elastin. Pease and Paule (1960), using phosphotungstic acid staining, observed 200 \& elastinlike units scattered in the arterial ground substance between the endothelium and the internal elastic lamina. Fyfe and Gillman (1964) observed fine, orcein-staining beads at the site eventually to be occupied by the internal elastic lamina in the 11-20 day old mouse aorta. With increasing age the beads appeared to fuse into larger masses, progressing from inner to outer lamina. Because of the location of the elastin beads, both Pease and Paule (1960) and Fyfe and Gillman (1964) concluded that the endothelium may secrete elastin. The production of elastin or an elastin precursor is an indirect, but important, contribution to the elastic properties of the arterial wall.

Burton (1954) pointed out that the capillary endothelium does not require great intrinsic strength because the capillary radius is so small. Since the cylindrical wall of capillaries is subject to the law of Laplace, the circumferential distending force is equal to the product of the pressure -20 to $40 \mathrm{~mm} \mathrm{Hg}$, and the radius radius- 4 to $5 \mu$. However, In large arteries, both the pressure and radius are much greater.

Fung ot al (1966) indirectly assessed the elasticity of capillaries by determining the elastic modulus of capillary- 
free regions of rabbit mesentery subjected to a torsional stress. The elastic modulus was taken as an indication of the environment of capillaries in other regions of mesentery which do contain capillaries. The authors then calculated the elastic properties of the capillary endothelium ifs a) a liquid-filled endothelial cell was assumed; and, if b) a gel-filled endothelial cell was assumed. A liquid will shear under stress, so in the case of a liquid-filled cell, the entire resistance to deformation must reside in the membrane. The calculated elastic modulus of the membrane of such a liquid-filled cell was about 100 times that of the published values (Bergel, 1961) for the oetl as a whole. Because of this obvious disparity, the authors rejected the hypothesis that the cells are liquid-filled. Instead, they tentatively. accepted the hypothesis that endothelial cells behave as a membrane-enclosed gel. Since the surrounding mesentery is also 'a gel, the authors suggested that the microcirculation might be viewed, in terms of mechanical properties, as a set of tunnels in a continuous, gelatinous environment. If the composition and orientation of the components of the mesentery and endothelial cell are similar, then the gelatinous capillary wall must manifest mechanical properties that resemile those measured for the surrounding mesentery. The elastic modulus of the mesentery was found to be alinear, commencing at about $3.0 \times 10^{6}$ dynes $/ \mathrm{cm}^{2}$ at minimum 
strains, and rising convex to the extension axis. If it is further assumed that the capillary and large arterial endothelium are comparable, then it may be concluded that the elastic properties of the large artery endothelium per se, resemble the elastic properties of the arterial wall as a whole (using the arterial data of Bergel, 1961). A complication in this comparison is the fact that both theproperties and the degree of strain of individual cells of the arterial endothelIum may differ from those of the capillary endothelium. The alinearity of the elasticity curve makes the degree of strain a relevant consideration. In any case, inspection of the wall of large arteries indicates that the endothelium represents a small percentage of cross-sectional area. It is probable, therefore, that the influence of the mechanical properties of the endothelium, whatever they are, is not very great. 2. Fibroblasts. Fibroblast cells are found in the arterial wall, mostly in the adventitia. These cells are believed to be responsible for the manufacture of collagen and ground substance. Light microscope studies of subcutaneous tissues in tissue culture (Bloom and Sandstrom, 1935) and in healing wounds in situ (Stearns, 1940) reveal that roticular fibers appear only at the outer surface of invading fibroblasts. Electronmicroscopic studies (Porter and Vanamee, 1949; Yardley et al, 1960) also show that these fibers appear only 
extracellularly. They also indicate that these fibers exhibit the typical banded appearance of collagen.

Histological studies have also demonstrated that Irritation of tissues with turpentine (Gersh and Catchpole, 1949) or quartz dust (Curran, 1953) causes a gathering of fibroblasts and a proliferation of a material which resembles ground substance. This material stains Hale-positive, indicating the presence of ground substance, and this staining reactfion is abolished if the tissue is pretreated with hyaluron(idase (Curran, 1953; Grossfield et al, 1955). Finally, autoradiographic studies indicate that fibroblasts incorporate $355_{4}$ into sulfated mucopolysaccharides (Curran and Kennedy, 1955; Kennedy, 1960).

No experiments to evaluate the mechanical properties of fibroblasts have been reported, but the absence of intracellular fibrous elements suggests that these cells do not exhibit collagen-like resistance to deformation. The ability of fibulasts to rapidly migrate to sites of tissue damage attests to their deformability. The viscosity of the cells is also unknown. If fibroblasts secrete the constituents of the ground substance in monomeric form, then it is not likely that the cells exhibit great viscosity. In any case, fibroblasts, like endothelial cells, represent a small percentage of the total arterial wall. Their great influence on arterial 
olasticity is undoubtedly an indirect one, ie., by the secretion of the connective tissue components.

3. Smooth muscle. a. Morphological Appearance and Contractile Proteins. Electronmicroscopic studies of various mammalian smooth muscles indicate the presence of bundles of filaments, usually, but not always, oriented parallel to the long axis of the cells. These bundles do not show the banding or alternating dark and light regions seen in skeletal muscle. Myofilaments of two diameters have been described (Weinstein and Ralph, 1951; Needham and Schoenberg, 1964; Lane, 1965) but all of these authors differ as to the diameters observed. They do agree, however, that at least one filament is about the size of actin. Panner and Honig (1967) studied ultrathin sections of smooth muscle. They found only one size of filament, about $40 \&$ in diameter, and argued that previous reports of multiple size filaments may have resulted from superimposition of filaments in thicker sections. $X$-ray diffract) tion studies on dried, wet, relaxed, contracted and glycerol extracted smooth muscle (Elliott, 1964, 1966) also showed reQflections similar to those obtained from $x$-ray diffraction studies of suspensions of extracted actin (Astbury et al, (1947).

Studies have been undertaken on the longltudinal dayer of mouse jejunum in various stages of contraction 
(Lane, 1965). With increasing degrees of contraction the individual muscle cells gradually changed from an elongated, spindle shape to a more rounded, ellipsoid configuration. The cells also exhibited invaginations of the membrane at the regions of the membrane in contact with the myofilaments. Such attachment regions have also been described in arterial smooth muscle (Pease and Molinari, 1960). Presumably the invaginations of the plasma membrane of jejunal smooth muscle cells reported by Lane (1965) result from shortening forces exerted by the myofilaments. The attachment areas and the associated regions of membrane deformation were not restricted exclusively to the poles of the cells.

Frequent, irregularly-spaced dense bodies in the cytoplasm have been noted (Schoenberg, 1958; Prosser et al, 1960; hanson and Lowy, 1961; Rhodin, 1962; Lane, 1965; Panner and Honig, 1967). These dense bodies appear to be attachiment points for actin filaments and exhibit stellate radiations of actin in electronmicrophotographs of homogenized, fixed, stained smooth muscle material (panner and Honig, 1967). These authors noted that strong internal forces must be present In the dense body-actin complex in order for it to withstand homogenization. They suggested as others had, that the dense bodies may be homologous to the Z-line structure found in skeletal muscle. Such a view is very attractive, but it is 
lpossible that the dense bodies observed in intact cells and in preparations of homogenates are not identical; those obtained from homogenates may be the result of random polymerization of actin.

Studies on contractile filaments have been performed on homogenates of smooth muscles (Hanson and Lowy, 1964; Rü8gg et al, 1965; Panner and Honig, 1967). Thick filaments corresponding to myosin have never been observed in these preparations. However, Hanson and Lowy (1964) were able to lsolate myosin-like structures from the supernatant of ultracentrifuged pregnant uterus. A rod-and-ball structure, similar to that described for synthetic skeletal muscle myosin (Huxley, 1963) was found. Panner and Honig (1967) combined urkey gizzard smooth muscle homogenates with rabbit skeletal woscle H-meromyosin. The synthetic acto-Hameromyosin that resulted gave the appearance of smooth muscle actin with small flaments projecting diagonally outward in the form of arrowpeads. These arrowheads appeared to be parallel over most of the length of actin. They were 30-50 \& wide and $450 \AA$ long. If sufficient H-meromyosin was present, the actin was virtually covered with these parallel arrowheads.' Similar structures, Ith relatively few arrowheads, were also observed in homogentes of smooth muscle to which no skeletal muscle extracts d been added. These experiments indicate that smooth muscle 
actin can, and probably does, combine with Hameromyosin. It also suggests, in spite of electronmicroscopic and $X$-ray diffraction data, that smooth muscle probably does contain a protein which gives myosinalike reactions. Needham and Williams (1963) were able to combine smooth muscle actin with skeletal muscle myosin, and a myosin-like protein from smooth muscle with actin obtained from skeletal muscle. Both of these combinations gave viscous, actomyosin-like reactions. It may be concluded from these various lines of evidence that myosin is present in smooth muscle, but exists in a disaggregated, perhaps soluble form (Rúlegg et al, 1965; Panner and Honig, 1967).

In order to separate actin and myosin from skeletal muscle, Huxley(1963) found it necessary to chelate calcium and provide high concentrations of ATP and magnesium. $\theta y$ contrast, Panner and Honig (1967) were able to isolate actin from smooth muscle homogenates with just a buffer solution, even in the presence of low concentrations of calcium. It appears, therefore, that actin-myosin bonds in smooth muscle are more easily broken than those in skeletal muscle.

Correlated with this viow are the reports of Needham and Schoenberg (1964) that uterine actomyosin exhibits only about one-tenth the ATPase activity of skeletal muscle actomyosin. Similarly, low calcium-ATPase activities were 
found by Barany et al (1966) for chicken gizzard myosin. However, following trypsin treatment to obtain $H_{-}$and L-meromyosin, both uterine (Needham and Schoenberg, 1964) and gizzard (Barany et al, 1966) acto-H-meromyosin ATPase activity was greatly increased, approaching that of skeletal muscle. Bohr ot al (1962) studied the ATPase activity of proteins extracted from hog carotid artery media in .6M potassium chloride solutions. The ATPase activity of this preparation was also found to be lower than that extracted from skeletal muscle. It was apparant, however, that some of the protein was bound to the insoluble portion of the extract because this portion showed considerable ATPase activity. Gaspar-Godfroid (1964) also extracted bovine carotid artery proteins, but in $.05 \mathrm{~m}$ potassium chloride. The protein extracted at this ionic strength showed ATPase activity equivalent to that of skeletal musclo.

Filo et al (1963) found that actomyosin extracted from hog carotid arteries in $.05 \mathrm{M}$ potassium chloride exhibited many of the typical properties of skeletal muscle actomyosins 1)superprecipitation; 2) reversible viscosity changes with the addition of ATP; 3) ATPase activity which was influenced by calcium, magnesium, and ionic strength. However, unmodified extracted arterial actomyosin exhibited high solubility and did not show superprecipitation. It was the investigators' 
opinion that this high solubility was the result of either a reduction in bound calcium, or the presence of an unusually effective solubilizing factor. Addition of calcium chloride permitted superprecipitation, suggesting that the calcium chloride somehow inactivated or saturated the proposed relaxing factor. A similar opinion was offered by Briggs (1963) to explain the high solubility of uterine actomyosin. The observation that trypsin hydrolysis greatly increases myosin-ATPase activity (Needham and Schoenberg, 1964; Barany et al, 1966) suggests that L-meromyosin might be the locus of such a solubilizing factor. A different interpretation has been offered by Laszt (1961) and Hamoir and Laszt (1962), who isolated contractile proteins from arterial and uterine smooth muscle at low lonic strengths. According to these authors, two proteins are present in smooth muscle; the usual actin and myosin, and a unique "tonomyosin". This protein is believed to be soluble under normal ionic conditions, but is thought to precipitate as "insoluble tonoactomyosin" with elevated intracellular potassium concentrations. According to the authors, intracellular precipitation of tonoactomyosin underlies tonic contractions. Conti at al (1964) held strips of bovine carotid artery under constant loads, treated them with $80 \mathrm{~mm}$ potassium chloride and prepared them for electronmicro8copy. Published microphotographs supposedly show a network 
like array of intracellular filaments, and decreased area of the contracted cells. Electrical stimulation or epinephrine treatment caused shortening, but failed to produce the formation of a network, or decreased muscle cell area. According to Laszt (1964), syneresis resulting from the precipitation of the soluble actomyosin is the cause of the reduction in cell cross-sectional area. However, neither the electronmicrophotographs nor Laszt's measurements of small changes in cross-sectional area and density are very convincing. Furthermore, Schoenberg et al (1966) treated extracted bovine carotid tonoactomyosin with ATP to form an insoluble superprecipitate. Excellent electronmicrophotographs of the preparation showed only actin-size filaments.

The present status of knowledge of smooth muscle contractile proteins is best summarized by saying that actin is fibrous; soluble and electronmicroscopically discernible, while myosin or a myosin-like protein may exist in a disaggregated form.

b. Membrane and Coupling Phenomena. The development of microelectrodes has permitted the measurement of resting membrane potentials in vascular smooth muscle. Intracellular recordings of smooth muscle in large elastic arteries reveal a resting potential of about $-40 \mathrm{mV}$ in turtio aorta (Roddie, 1962), -49 MV in sheep carotid artery (Keatinge, 1964) 
and - $52 \mathrm{mV}$ in rabbit pulmonary artery (Su et al, 1966; Bevan fand Verity, 1966). These may be contrasted with oscillating nembrane potentials of veins, some arterioles and visceral nuscle. The mean resting membrane potential in taenia coli is (-51 mV (Bulbring, 1954; Holman, 1958). These values are considerably lower than the resting potential for skeletal muscle. Some of this difference may be attributed to the presence of different intracellular ion concentrations in smooth and skeletal muscle. Intestinal muscle (Barr, 1959), taenia col1 (Durbin and Monson, 1961), and frog stomach muscle (Armstrong, (1964) have been shown to contain higher intracellular concentrations of sodium, and lower intracellular concentrations of potassium, than skeletal muscle (Conway, 1957). However, the resting membrane potential cannot be fully accounted for by the existing concentration gradients, because the resting membrane potential is about $20 \mathrm{mV}$ less than predicted by the Nernst-Goldman equation (Goodford and Hermansen, 1961). Both fourbin and Monson (1961) and Goodford and Hermansen (1961) preported that the rate of labelled sodium flux is extremely high, the half-time for $95 \%$ transfer is only about 30 seconds. Kuriyama (1963) studied the influence of altered extracellular soodium, potassium and chloride concentrations on the resting fmembrane potential in taenia coli. The curve relating the log fof the external potassium concentration to the resting membrane 
potential exhibited a slope of only $38 \mathrm{mV}$ per ten fold change in potassium concentration. This may be compared with the $44 \mathrm{mV}$ per ten fold change in potassium concentration in skeletal muscle fibers observed by Ling and Gerard (1950). When non-penetrating tris ${ }^{+}$was used to replace extracellular sodium, Kuriyama found a slope of $44 \mathrm{mV}$ per ten fold change in extracellular potassium concentration. Kuriyama interpreted these results as an indication of greater sodium permeability in smooth muscle than in skeletal muscle. Still unresolved, however, is the question of whether the resting membrane fluctuations cause, or are themselves caused by the alterations in membrane permeability. Although the smooth muscle of large arteries does not exhibit oscillating membrane potentials, it does exhibit a lower resting membrane potential than skeletal muscle and nerve. It may be surmised that this is correlated with intracellular concentrations of potassium and sodium which differ from those found in skeletal muscle. Increased permeabilities to these ions may also exist, but recording of membrane potentials during experimental manipulation of ion concentrations has not been performed in smooth muscle. Action potentials have been recorded from smooth muscle cells. Bulbring (1954) demonstrated, in taenia coll, that stretch was associated with an increase in action potential Prequency and an increased contractile tension. Action poten- 
tial frequency correlated better with tension than with length, because following stretch, the increased electrical activity declined as the tension declined in spite of continued elongation. It would therefore appear that the contractile element is coupled in series with an element which, when stressed, can alter ion movements into and out of the cell. A likely candidate for this role might be the membrane itself;

Keatinge (1964, 1966a,b) has recorded a slight hypopolarization of sheep carotid artery smooth muscle in response to sympathetic nerve stimulation or the application of constrictor drugs. Only occasionally were actual spikes recorded. Su et al (1964) similarly recorded strong contractions from rabbit pulmonary artery strips with little or no change in membrane potential following sympathetic nerve stimulation or treatment with norepinephrine. Both hypopolarization and mechanical contraction were seen following treatment with high concentrations of potassium chloride. Evans et al (1958) recorded the membrane activity of taenia coli. Continuous depolarization was observed when the tissue was bathed in potassium chloride or Ringers solution in which the sodium had been replaced with potassium. Neither stretching nor electrical stimulation of this tissue elicited mechanical contractions, but application of acetylcholine resulted in prompt, reversible contractions. Waugh (1962) bathed perfused mesenteric arteries 
in potassium sulfate solutions. Although membrane potentials were not recorded it was presumed that depolarization had taken place. Treatment of these preparations with epinephrine gave typical contractile responses. Unlike other innervated arteries, non-innervated umbilical artery (Davignon et al, 1965) showed strong contractions in response to stretch. Contraction could also be elicited by treatment with angiotensin or serotonin. Response to anglotensin was gradually depressed by tachyphylaxis, and response to serotonin was blocked by phenoxybenzamine. However, myogenic responses could still be elicited under these conditions of unresponsiveness. Evidently, these responses are mediated by separate mechanisms.

A number of studies on smooth muscle have demonstrated a mixed population of cells. Intracellularly applied currents in taenia coli (Kuriyama and Tomita, 1965) and vas deferens (Bennett, 1967) demonstrated two classes of cells. One group exhibited action potentials in response to depolarizing currents, while the other group exhibited only electrotonus. The passively responding cells were about three times as numerous as the active cells. In the case of the vas deferens, hypogastric nerve stimulation gave similar results. Intracellular recordings of arteriolar smooth muscle (Funaki, 1961) also showed two populations; one group of cells exhibited spontaneous electrical activity, and the other was persistently 
polarized at the resting level. Siggens and Berman (1967) also reported intracellular microelectrode studies, on arteriolar smooth muscle. Stimulation of vasomotor nerves caused mechanical contraction in all arterioles studied, but caused the development of an action potential in only 25 of the 103 arteriolar cells studied. It appears, therefore, that in smooth muscle, electrical activity may be an important intervening step, as in the contraction which occurs in response to stretch. However, it may also be entirely bypassed, as in the contractile response to neurohumoral agents.

Sandow (1966) has summarized the current concept of excitation-contraction coupling in skeletal muscle. Once initiated, membrane depolarization radiates inward along the $T_{-}$ tubules, depolarizing the lateral cisterna of the sarcoplasmic reticulum. This structure releases ionic calcium, an agent which greatly facilitates the polymerization of actin and myosin. Following the induction of contraction, ionic calcium is re-bound, curtailing its facilitatory action on actomyosin. The agent responsible for this re-binding process has been termed "relaxing factor". It is carried out by a vesicular fraction of the sarcoplasmic reticulum (Muscatello et al, 1962) which is probably manufactured by the sarcoplasmic reticulum (Briggs and Fuchs, 1960). Recently Lee et al (1966) demonstrated that the calcium-binding capacity of suspensions of 
sarcoplasmic reticulum is briefly and reversibly inhibited by the passing of an electric current through the suspension. Palmer and Posey (1967) showed that the introduction of sodium ions also inhibits the calcium-binding capacity of sarcoplasmic reticulum suspensions, and studies with labelled calcium revealed an actual release of bound calcium.

While the excitation-contraction process is now well established for skeletal muscle, the mechanisms of activation of contractile proteins in smooth muscle are poorly understood. Electronmicroscopic studies of smooth muscle indicate that only a rudimentary sarcoplasmic reticulum exists. (Mark, 1956). Electro-mechanical latency is too brief in skeletal.muscle to be accounted for by diffusion of calcium from the membrane to the contractile proteins. However, the long latency in smooth muscle can readily be explained by such diffusion (Peachey and Porter, 1959). Where smooth muscle subserves rapid movements, as in the swimming motions of amphioxus, the contractile system has been found to be laid out in flat, thin sheets, $1 \mu$ thick: and closely epposed to the membrane (Peachey and Porter, 1959).

Although diffusion of ionic calcium or other activation substances may account for activation of mechanical contraction in smooth muscle, the location of the relaxing factor remains to be determined. Filo et al (1963) suggested 
that the relaxing factor in carotid arterial smooth muscle may be firmly bound to the contractile protein itself, so firmly in fact, that it is apparantly extracted with the protein. Briggs (1963) came to the same conclusion in his studies on uterine muscle. The nature of the relaxing factor in smooth muscle is unknown.

Normal energy production in taenia coll can be inhibited by exposure to metabolic inhibitors (Bulbring and Lullman, 1957) or, to a glucose-free medium (Axelsson and Bulbring, 1961). Such inhibition was found to cause a marked increase in membrane action potential frequency and a transient increase in tension followed by a gradual decline in contractile tension. These effects were prevented if the taenia coli was bathed in a calcium-rich medium (Bulbring and Kuriyama, 1963). The authors suggested that a decrease in metabolic energy may permit a release of bound calcium from the membrane, and that this in turn permits increased permeability to sodium and potassium ions; elevated calcium in the extracellular fluid would thus replace unbound, lost calcium, maintaining normal permeabilities and sufficient calcium levels to sustain contractile mechanics. These experiments suggest that the cell membrane itself may be the important site of calcium-binding, as was suggested earlier for neurogenic contractions of smooth muscle. The protein-bound relaxing factor of Filo et al (1963) and 
Briggs (1963) may be a separate relaxing factor; it may be the ATPase-inhibitor uncovered by Needham and Schoenberg (1964)

c. Mechanical Properties. 1. Active tension.

Smooth muscle contraction differs from that of skeletal muscle in several respects. Both the stimulus-response latency and the duration of tension development are many times longer for smooth muscle than the comparable events for skeletal muscle. Smooth muscle also exhibits a slow increase in contractile tension. In smooth muscles of Holothuria (Hill, 1926) quick stretches and quick releases revealed that the speed of contraction is only $1 / 100$ that of frog skeletal muscle. The fall of the tension to the level characteristic for the new length was very similar in its time course to the rise in tension produced by the stretch. In smooth muscle of Mytilus (Abbott and Lowy, 1953) electrical stimulation ovoked contractions as slow as $1 / 40$ to $1 / 20$ the velocity of frog skeletal muscle. In these and other species (Pecten and Helix - Bozler, 1936) smooth muscle contraction consists of a slow, gradual increase in tension followed by a prolonged, assymptotic decline in tension. The contractile phase of this response may be elicited by electrical or chemical stimulation or by stretch (Bulbring, 1954). If the muscle is spontaneously active, stretch may inhibit contractile tension. However, myogenic responses to otretch are not seen in multi-unit smooth muscles such as those 
of the carotid artery or nictitating membrane (Burnstock and Prosser, 1960). Similarly, Kosan and Burton (1966), found no increase in oxygen consumption in segments of dog femoral arteries subjected to circumferential stretch.

The assymptotic decline which follows the tension increase has been analyzed by a number of investigators. Bozler (1936) noted that the time course of the decline following an isometric contraction almost exactly parallels the tension decline following an elongation-induced tension increase. Abbott and Lowy (1958a) reinvestigated this comparison with more sensitive apparatus, and concluded, contrary to Bozler, that these reflect fundamentally different events. Zatzman et al (1954) studied the time course of stretch-induced stress relazation of arteries. These authors attributed the viscous fall in tension to the muscle component of the wall because it was most prevalent in arteries cóntaining large amounts of the muscle, and because it was largely abolished in arteries in which the muscle was presumed inactive after heating.

The active force developed by striated muscle increases with length, reaches a maximum at an ideal length, and declines with further elongation. This phenomenon is believed to result from the number of actin-myosin bonds that are formed, and this is believed to depend upon the longitudinal overlap 
and the axial proximity of actin and myosin filaments (Huxley 1960). In length-tension studies, Evans and Hill (1914) observed that the amount of heat produced by contracting skeletal muscle closely paralleled the tension that was developed. This indicates that the length-active tension relationship reflects some underlying force-producing process, and is not just the result of changes in the efficiency or force vector of the contractile system. Contractile tension has been correlated with sarcomere length in skeletal muscle (Carlson et al, 1961) and cardiac muscle (Spiro et al, 1964), but in smooth muscle no Z-lines are present and no distinct sarcomeres can be defined. In spite of the absence of these structures, there are several reports that smooth muscle exhibits a length-tension curve which resembles the length-tension curve for skeletal and cardiac muscle.

Winton (1926) systematically extended dog retractor penis muscle to various lengths. Electrical stimulation at each length produced discrete isometric contractions. the length-tension curve obtained by this procedure resembled the length-tension relationship recorded for skeletal muscle, but exhibited maximum active tension at very long lengths, corresponding to strains of 3.5 .

Hampel (1934) stimulated cat nictitating membrane In situ with electrical stimulation and with intravenous in- 
jections of epinephrine. Length-tension curves were obtained at various stages during extension, and during release. Peak active tensions were found to shift according to the procedure. Active tensions recorded during extension occurred at short lengths, and were of greater strength than those recorded during release of extension. The strength of contraction was about 11.5 grams, but the cross-sectional area was not determined. Csapo (1954) electrically stimulated uterine muscle in vitro. A broad peak active tension was recorded which approximately corresponded to the length of the tissue at rest. The actual tension developed depended upon hormonal conditions Abbott and Lowy (1953, 1958a) recorded contractile properties of Mytilus and Helix smooth muscles. When stimulated electrically, these tissues exhibited length-tension with peak active tension occurring at very long lengths. The active tension described a rather flat curve with a very rounded peak. The entire curve was shifted if the muscles were extended overnight between successive stimulations. Peak active stresses ranged from 0.5 to $4.5 \mathrm{~kg} / \mathrm{cm}^{2}$. In another paper, Abbott and Lowy (1958b) noted that the force-velocity diagram for these molluscan muscles could be approximated by Hill's load-velocity equation for skeletal muscle. Winton (1926) obtained in vitro length-tension curves for pregnant guinea pig uterus muscle stimulated with 
pituitary extract. The tissue was stretched, treated with the hormone, then washed before further extension. The active length-tension curve obtained from this preparation exhibited a peak at a strain of about 4.0 .

Brocklehurst (1926) recorded a length-tension curve for cat ileal muscle. The intestinal segment was permitted to relax and then was stimulated to contract with histamine. Tensions as high as, $200 \mathrm{gm}$ were recorded, but crosssectional areas were not given. The drug was then washed out. and the tissue segment was extended. Data obtained by these experimenters gave two length-tension diagrams. When the tissue was stimulated at successively shorter lengths a peak was obtained at very large strains. When the tissue was stimulated at successively longer lengths, a peak was obtained at very low strains. The directional difference was explained on the basis of viscosity and the possibility of stretching of non-muscular elements in the intestine, but neither explanation was developed.

Ducret (1931) studied the influence of initial load on active tension developed by epinephrine-treated rings of mesenteric artery. Ducret found that the drug-treated ring developed a maximum of $1.5 \mathrm{gm}$ active tension per quarter $\mathrm{mm}^{2}$ of cross-section of smooth muscle. The dimensions were obtained from measurements of histological sections and therefore 
he of limited applicability to unfixed, hydrated vessels. Although Ducret reported his data in terms of initial load, the load can be alinearly related to length. Maximum contractile tensions were found when the tissue was initially loaded with 2 grams before epinephrine treatment.

Speden(1960) obtained length-tension diagrams of spiral strips cut from sheep mesenteric arteries. Contraction was induced by treating the strips with norepinephrine. A peak active tension of $3.0 \mathrm{gm}$ was observed at a strain of 1.25 . Cross-sectional areas were not determined.

Sparks and Bohr (1962) studied strips cut from dog mesenteric arteries. Contraction was elicited by either electrically stimulating tissue strips, or treating them with epijephrine. Peak active tensions were recorded at lengths that were 55 to $138 \%$ above the length at which resting tension initially appeared. Maximum active tensions ranged from $0.9^{\circ}$ to 5.3 grams.

Hinke and Wilson (1962) studied the blood flow through an isolated, cannulated $550 \mu$ rat tail artery. The resistance, and therefore the radius, was calculated from the pressure gradient and flow of Krebs-Henseleit solution. length-tension diagrams were obtained under resting conditions and during perfusion with norepinephrine. Active tension 
curves were obtained which showed no peak, but rose monotonically. If the pressure was elevated in constricted vessels, the arteries maintained their constricted size, describing a virtually infinite elastic modulus.

Apter (1966) obtained length-tension curves for rings of pulmonary artery treated with Ringer solution, noosynephrine and later with a chelating agent. The difference between the tension following administration of Ringer solution and EDTA provided an active tension curve which was very flat and with iittle peak. Maximum tension was bout 10 grams. When the same ring was treated with neosynephrine, a steep, monotonic, unpeaked curve was obtained. These curves were plotted from data acquired by extending the arterial segment. Lundholm and Mohme-Lundholm (1966) cut mesenteric arteries longitudinally and rolled the tissue out into flat sheets. The longitudinally cut edges were mounted in plastic holders and extended as strips under load. A length-tension diagram was obtained by extending or releasing the specimen, treating it with epinephrine, washing this out, and then changing the length. The specimens were also studied in the untreated state, and following treatment with monoiodoacetic acid. The thickness of the specimen was measured with calipers. Maximum contractile tension was found to be $2000 \mathrm{gm} / \mathrm{cm}^{2}$. 
Uchida et al (1967) studied mesenteric and cerebral arteries as small as 50 to $250 \mu$ diameter using the constantflow perfusion technique of Hinke and Wilson (1962). Addition of norepinephrine to the perfusate caused an increase in resistance: The magnitude of the resistance increment increased with a rise in perfusion pressure up to $25 \mathrm{~mm} \mathrm{Hg}$. It remained unchanged in the $25-60 \mathrm{~mm} H \mathrm{Hg}$ pressure range, and then decreased with higher pressures.

2. Resting tension and tone. Bozler (1936) studled the resting tension of smooth muscles of the snail by recording stretch-induced stress relaxation over a wide range of lengths. The static stress achieved following relaxation was plotted as a function of length. It may be that this parameter is more residual tone than resting tension. However, Bozler found the familiar resting tension hyperbola, but it was very flat, steepening only at strains of almost 4.0 . The significance of this curve as a plot of resting tension is difficult to assess. The concept of "resting length", as it is used with respect to skeletal muscle, is virtually without meaning when it is applied to tremendously extensible smooth muscle. The classical picture for skeletal muscle (Carlson et al, 1961) is that resting tension does not appear until the resting length 18:exce日ded. Cardiac papillary muscle (Spiro et al, 1964), on the other hand exhibits resting tension at 
lengths well below that at which maximum contractile tension is developed. Smooth muscle develops negligible resting tension until extraordinary strains are achieved, but it is at these very great strains that the peak in active tension is encountered. This implies that the contractile system may be involved in the apparent "resting" tension.

Resting tension of mammalian smooth muscle is difficult to evaluate because this tissue is inevitably surrounded by large amounts of connective tissue. From a functional point of view it is reasonable to consider the resting tension of the tissue as a whole. This is actually what was done to obtain the resting tension in skeletal muscle. If this approach is taken with mammalian smooth muscle, length-resting tension characteristics will probably be obtained which closely resemble the load-extension diagrams of elastin and collagen. One characteristic of smooth muscle is its ability to resist distention for long periods of time. Winton (1926) stated that "skeletal muscles are often described as functionally isometric, while unstriated organs approach more closely to isotonic conditions". Although the maintenance of tone requires metabolic energy, it does not demand the amount of energy requirediforaphasicucootraction. Carbohydrate depletion or inhibition of carbohydrate metabolism by anoxia or metabolic poisoning in longltudinal muscle of rabbit intestine 
(Neukirch and Rona, 1912; Prasad, 1935; Furchgott and Schorr, 1946) and isolated rings of carotid artery (Sudhof, 1950) resulted in decreased smooth muscle tone. However, maintained tone in the adductor muscles of bivalve molluscs (Conheim and vonUexkull, 1911) and uterus, vagina and intestine of rabbit, Guinea pig and cat (Evans, 1923) caused no increase in oxygen consumption. Bulbring (1953) reported similar results for the oxygen consumption of both isometric and isotonic contractions of guinea pig taenia coll. Similarly, Parker (1922) reported that the quantity of carbon dioxide released by day-long, tonid contractions of the sea anemone was not augmented, except for a $25 \%$ increase at the onset of contraction. Lundholm and Mohme-Lundholm (1962) have reported similar findings for arterial strips during epinephrine-induced isotonic shortening. After the initial rise in tension, energy production fell to precontraction levels, in spite of the persistence of isotonic tone. Lundholm and Mohme-Lundholm (1965) also found that during the tension-increasing phase of epinephrine-induced isometric contractions of arterial strips, the energy utilized was 3 to 4 times that of the subsequent phase of sustained elevated tension. ATP, ADP and creatine phosphate have been measured in iodoacetate-treated carotid arterial strips (Daemers-Lambert and Roland, 1967). A 20-second phasic contraction consumed $.35 \mu \mathrm{M} \mathrm{ATP/gm}$ of carotid tissue, while a 
tonic contraction maintained for 1 minute consumed $.22 \mathrm{\mu m}$ ATP/gm/minute. These studies also showed that phasic contractions consume much more energy per unit time than tonic contractions. Lundholm and Mohme-Lundholm (1960) observed that agents such as histamine and barium ion increase the tone of arterial strips with no increase in oxygen except during the initial phase of tension increase. However, if the preparations were first depleted of energy stores then neither histamine nor barium would increase tone. It thus appears that two distinct processes are involved in the development and maintainance of tone. The first is clearly an energyconsuming contractile response, and the second appears to be a passive, holding mechanism.

Three general hypotheses have been presented to explain tone. One hypothesis views tone as the product of a passive "ratchet" mechanism, arranged in parallel with the contractile elements (Cohnheim and von Uexkull, 1911). A second hypothesis views tone as the product of reversible crystallinization of a muscle protein that is distinct from actomyosin. Johnson (1962) proposed that this function may be fulfilled by paramyosin, arranged in parallel with phasically contracting elements in molluscan smooth muscle. A third hypothesis viows tone as a sustained polymerization of actin and myosin, due either to a decrease in avallable ATP (Bozler, 
1957), or perhaps to a slow-acting relaxing factor. A decrease in available ATP probably can be ruled out as a possibility because energy depletion in smooth muscle results, not in rigor, but in flaccid relaxation (MacWilliam, 1902). A slow-acting relaxing factor has yet to be evaluated. In line with this possibility is the suggestion of Lowy and Millman (1959) that a low rate constant of actin-myosin depolymerization might be the factor underlying tone. Finally, the possibility that different or even multiple mechanisms may operate in different smooth muscles must also be considered. D. Mechanical Properties of Arteries. 1. Relaxed Vessels. Early studies on the mechanical properties of arteries were descriptive and largely concerned with pathological considerations. Roy (1880), Israel (1886) and Herringham and Wills (1904) employed strips cut from arteries, usually from the aorta. When these preparations were extended with weights, it was noted by all of these experimenters that the greatest amount of lengthening occurred with the addition of weights to unextended arterial tissue. When weights of the same magnitude were added to extended arteries, decreasing increments of extension were observed. Roy and Israel also noted that transverse strips were less extensible than longitudinal strips. Poletbnow (1868) and Herringham and Wills (1904) correlated the presence of sclerotic lesions with decreased 
extensibility.

Marey (1880), Roy (1880), Grunmach (1888), MacWilliam, (1904), Strasburger (1907), Bramwell ot al (1923b), and Hurthle (1923) performed pressure-volume experiments on excised cylindrical arterial segments. Fluid was injected into these vessels and the pressure developed at each level of capacity was noted. This method more nearly resembled in situ conditions, and the results obtained confirmed the findings of studies on strips that distensibility decreases with extension. Bramwell et al (1923a) compressed the arms of living humans with a pressure cuff to control distal transmural pressures. Arterial pulse wave velocities were measured at various levels of compression permitting calculation of extensibility by application of a modified form of the Moens-Korteweg equation. The results of these studies also showed that extensibility decreases with increased transmural pressures.

One of the first systematic analyses of the tensibility of arteries was presented by Reuterwall (1921) who performed load-extension studies on strips of arteries, ligamentum nuchae and collagenous tendons. Because of the biphasic nature of the arterial extensibility curve, Reuterwall suggested that the elastin and collagen of the wall might be arranged in parallel, the elastin bearing the load at moderate extensions, and the collagen bearing the load at larger extensions. 
Reuterwall also described histological observations which revealed that collagen was "curled" or slack in retracted vessels. Light and electronmicroscopic studies of vessels which were systematically fixed at various distending pressures (Wolinsky and Glagov, 1964), supported these early histological observations. Krafka (1939) extended the analysis of vessel distensibility in noting that the elastic moduli of strips of aortae ( 1.5 to $1.9 \times 10^{6}$ dynes $\left./ \mathrm{cm}^{2}\right)$ were consistently lower than that of elastin-rich ligamentum nuchae (about $4 \times 10^{6}$ dynes $/ \mathrm{cm}^{2}$. This observation led Krafka to conclude that a network arrangement of elastin must exist within the walls of arteries. When vessels were permitted to putrefy to remove elastin and any active muscle that might be present, a general rise in the olastic modulus was seen at all loads. Histological inspection verified a disintegration of elastin and a relative persistence of collagen. In some experiments on unputrefied vessels, Krafka separated the intact aorta into media and adventitia: At $100 \mathrm{gm}$ load, the elastic modulus of the media was just less than the modulus of the intact artery; at the same load the elastic modulus of the adventitia was 15 times greater than that of the intact artery. The adventitia is rich in collagen. Correlation between the presence of much collagen and a high elastic modulus of the adventitia supported the interpretation that it was predominantly collagen that was bearing the load Pollowing putrefaction of the intact vessel. Krafka also 
introduced an important methodological approach by using an incremental elastic modulus in place of the standard Young's modulus of elasticity. An incremental modulus is actually a series of approximations of the slope of a curvilinear stressstrain curve. This is a counterpart of Young's modulus, a single value describing the slope of a linear stress-strain curve.

Remington et al (1945) repeated Krafka's putrefaction experiments on isolated segments of aorta. After 15 days of putrefaction, pressure-volume curves throughout the pressure range paralleled those encountered at large extensions, 1.0. resembled those apparently dominated by collagen. After 22 days, the vessel easily ruptured, and histological inspection revealed that little intact collagen remained. Stress-strain Geurves of fresh thoracic aorta published by Remington (1957) exhibit elastic moduli of $1.7 \times 10^{6}$ dynes $/ \mathrm{cm}^{2}$ to strains of Kabout .70. After putrefaction, the elastic modulus of this had dropped to $8.6 \times 10^{5}$ dynes $/ \mathrm{cm}^{2}$. An even more elegant experiment of this type was performed by Roach and Burton (1957). These authors treated segments of human iliac arteries with unpurified trypsin to remove elastin, or formic acid to remove collagen and other materials. Franchini and DeRobertis (1951) had previously demonstrated in electronmicroscopic studies that trypsin does not hydrolyze collagen. Roach and Burton 
determined the tangential tension ( the product of pressure and radius) at each elongation in treated and untreated segments of human iliac artery subjected to volume distention. The slope of the tension-elongation curve throughout the distensibility range, following treatment with formic acid, paralleled the slope of the tension-elongation curve of untreated vessels at small to moderate distentions. After treatment with trypsin, the slope of the tension-elongation curve throughout the distensibility range paralleled the slope of the untreated vessels at large distentions. These data provide convincing evidence that the elastic characteristics of normal, relaxed vessels result from an interaction of elastin and collagen. Elastin dominates at low extensions, and collagen dominates at higher extensions. Evidence for the role of collagen also derives from studies on the influence of age on arteries. Many studies have indicated that the elasticity of human arteries increases with age (Roy, 1880; Herringham and Wills, 1904; Yater and Birkland, 1930; Wilens, 1937; Hallock and Benson, 1937): Roach and Burton (1959) correlated the tension-strain curves and histology of human iliac arterles of patients of different ages. The majority of histologicallydefined collagen fibers in young arteries were stretched only at large strains, and the strain at which the majority were stretched became progressively smaller with age. The strain 
at which stiffening of tension-strain curve occurred also shifted, correlating with the histological data. The aortae of rats (Hume, 1939) and rabbits (Saxton, 1942) show a decline in elasticity with age. It would be of interest to repeat Roach and Burton's (1959) comparison of mechanical behavior and histological appearance of the arteries of these animals.

The apparently parallel arrangement of elastin and collagen should set the limits of minimum and maximum elasticity. It might be predicted that the elasticity of the intact vessel would never be as high as pure collagen, as exemplified by tendon, and would never be as low as pure elastin, as exemplified by ligamentum nuchae. the first prediction has been verified, but the second prediction has not been found (Krafka, 1939). When the fibers are predominantly in a stretched state the elastic modulus of the whole arterial wall should be freater than that of elastin, but still less than collagen. This is because the elastin represents a portion of the crosssectional area which is less elastic than collagen. At low extensions collagen is apparently slack. This greatly complicates the analysis for it is difficult to assess the inPluence of a population of collagen fibers in various stages of "slackness". If the collagen fibers are predominantly in - slack state then the elastic modulus of the whole tissue chould be less than that exhibited by the elastin component 
alone. In this condition the slack collagen fibers offer little resistance to distention. Because the elastic modulus relates stress to strain, the increased "passive" cross-sectional area resulting from slack, and therefore extensible, collagen fibers should reduce the elastic modulus to a level below that of pure elastin. This appears to be the case (Krafka, 1939) but retraction of the elastin network into a random orientation at extremely small strains renders assessment of the influence of elastin and collagen almost impossible. In any case, these strains never occur in life.

Thermoelastic analyses of the intact arterial wall also have been performed. Roy (1880) placed a thermocouple on isolated aortic strips and coated this preparation with oil to prevent drying. When the load on extended strips was reduced, the strips retracted, and exhibited a decrease in temperature; when the strips were reextended, the temperature rose. Lawton(1954) measured the retractive force generated by aortic strips subjected to changes in temperature. An almost linear relationship was found between the temperature and the retractive force exerted. The slopes of these curves were approximately equal for circumferential, diagonal and longitudinal strips. Calculations indicated that the retractile force is largely entropic, increasing greatly at retracted lengths. Internal energy, an indication of crystallization, 
was found to remain essentially constant throughout the extensibility range, just as Hoeve and Florey (1958) had found for ligamentum nuchae. Identical entropic energy-to-length characteristics were found for intact aortic strips, and for aortic elastin extracted with alkali. This reaffirms the view that much of the behavior of the arteries may be accounted for by elastin. A marked increase in entropy found upon retraction of both the aorta and the extracted elastin suggests that retraction is correlated with configurational changes, as the elastin gradually assumes a more and more random orientation. The fact that strips cut at all angles from the aorta showed equal entropic energies suggests that the elastin in the wall also exhibits equivalent properties in all directions. The many in vitro studies performed on arterial strips and rings suffer from a failure to maintain appropriate geometry. When an artery is excised, it retracts to $60-70 \%$ of its in situ length (McDonald, 1960). When this retraction occurs the wall thickens, the intramural structures are reoriented and are permitted, perhaps, to retract into a slack state. These unnatural conditions do not represent in situ conditions, and the mechanical behavior obtained under such conditions probably does not reflect the mechanical properties of the same arteries in situ. Learoyd and Taylor (1966) studied arteries extended to various lengths, beginning with 
the excised, unextended length. A typical curvilinear circumferential elastic modulus was obtained. This shifted gradually as the artery was elongated in steps. Á progressively increasing stiffiess was observed at each radius as the artery was elongated.

It might appear that the ideal location in which to study arteries is in situ. Such studies have been undertaken on living dogs (Lawton, 1957; Peterson et al, 1960; Patel et al, 1960; Remington, 1962) and humans (Greenfield et al, 1965). However, in vivo conditions are unsatisfactory for studying mechanical properties of arteries because of the incessant pressure pulsations. These oscillations preclude the possibility of the artery ever achieving a static equilibrium state. Estimates of elasticity are also complicated by the effects of viscosity, by the accelerated mass of the wall, and by the many factors present which may influence smooth muscle activity. In spite of these shortcomings, in vivo studies do indicate that the conduit arteries exhibit small changes in dimension. Arterial diameter increases 2 to $8 \%$ over the course of the normal cardiac cycle, changing in phase with the pressure pulse. Length changes are in the order of $1 \%$, and these may occur either in phase or $180^{\circ}$ out of phase with the diameter changes. Bergel (1961) has described the only systematic in vitro study on the properties of arteries performed under conditions of appropriate geometry. Dog aortae, femoral, carotid 
and iliac arteries were excised and studied in air, at in situ length. The vessels were inflated from 40 to $240 \mathrm{~mm} \mathrm{Hg}$, while the diameter was simultaneously measured with a photoelectric device. Incremental elastic moduli were calculated from these pressure-diameter data. At low extensions, values of about $1.0 \times 10^{6}$ dynes $/ \mathrm{cm}^{2}$ were observed. The elasticity rose slightly with moderate extension, and then rose more and more steeply, but without a sharp inflection. Elastic moduli of 12.0 to $16.0 \times 10^{6}$ dynes/ $\mathrm{cm}^{2}$ were observed at extensions produced by $240 \mathrm{~mm}$ Hg pressure. The state of the smooth muscle in these arteries was unknown, but presumably it was relaxed. 2. Influence of Smooth Muscle on Mechanical Properties of Arteries. a. Shortening Behavior. It has been observed that activation of vascular smooth muscle causes a decrease in the diameter of conduit arteries. This has been reported where contraction was produced by direct stimulation of peripheral sympathetic nerves (Davis, 1964; Keatinge, 1966b), by hypothalamic stimulation (Weckman, 1960), by reflexes initiated by interruption of normal carotid sinus afferent nerve activity (Wiggers and Wegria), 1938; Gerova and Gero, 1967), by reflexes initiated by decreased environmental temperature (Haddy et al, 1957b) and by injections of catecholamines (Haddy et al, 1957a). Keatinge (1966b) found that stimulation of cervical sympathetic nerves caused a reduction in the dia- 
meter of the common carotid artery to $60-70 \%$ of control. Davis (1964) studied the outflow from a cannulated digital artery in dogs. He reported that sympathetic nerve stimulation caused a complete cessation of digital artery blood flow in 7 out of 12 animals. Haddy at al (1957b) cannulated lateral branches of large and small arteries, and large and small veins in intact, innervated dog limbs. The limb was supplied with arterial blood from a constant flow pump. The authors observed a general increase in resistance following injection of pressor agents. The large arteries contributed to the rise In resistance, but failed to maintain constriction for as long as the smaller vascular segments.

Length-tension curves have been obtained in vitro from studies on rings (Ducret, 1931) and strips (Speden, 1960; Lundholm and Mohme-Lundholm, 1966) cut from mesenteric arteries, and from perfusion experiments on small arteries (Hinke and Wilson, 1962; Uchida et al, 1967). Epinephrine and norepinephrine were used to stimulate the smooth muscle in these tissues. These studies were discussed in the section considerIng the active tension of smooth muscle. Burton (1951) has considered the constricting influence of contracting smooth muscle. According to Burton's analysis, the walls of arteries Can maintain equilibrium in purely passive state because of the restraining force of the connective tissue. He calls this 
"elastic tension". Additional stress, provided by active smooth muscle, upsets this equilibrium and causes a decrease in diameter. A decrease in diameter further increases the disequilibrium and leads, ultimately, to complete closure of the artery. Complete closure does not occur in large arteries, he reasons, because of the large amount of connective tissue present, which somehow prevents excessive constriction. Although never stated as such, this would mean that the length of connective tissue elements under zero net stress would be greater than would be found with zero transmural pressure. In other words, active energy must be required to compress the connective tissue elements down to obliterate the lumen. This point has mever been evaluated. Many pharmacological studies on arterial strips and rings have shown that addition of strong doses of epinephrine and norepinephrine causes shortening and thus mimic vasoconstriction. Barbour $(1912 a, b$,$) reported that$ epinephrine produced strong contraction only on transverselycut strips; longitudinally-cut strips exhibited only slight contraction. In situ studies on isolated segments of dog aorta (Alexander, 1953) indicate that local application of epinephrine causes contraction. Marked constriction was observed at pressures below $100 \mathrm{~mm} \mathrm{Hg}$, but little constriction was seen above that pressure. This appears to be at variance with the many observations of peak active tensions at very long lengthe.' 
b. Distensibility. Active arterial constriction is an important function of smooth muscle, but alterations of arterial resistance to distention may be even more important. Wezler and Boger (1936) assessed the distensibility of arteries in vivo by means of measuring the pulse wave velocity, using the Bramwell and $\mathrm{Hill}$ expression relating pulse wave velocity to pressure-volume distensibility. Injections of epinephrine increased arterial distensibility. Wiggers and Wogria (1938) recorded the diameter of the thoracic aorta in living dogs. Bilateral carotid occlusion produced an increase in arterial pressure which caused a passive increase in the diameter of the aorta. Subsequently, however, a secondary decrease in aortic diameter was observed. Aortic distensibility was increased during the constricted phase.

Lawton (1957) stitched beads onto the aorta and photographed the artery in vivo. Administration of norepinephrine produced a marked dfcrease in distensibility; administration of acetylcholine had just the opposite effect. Peterson et al (1960) measured the diameter of the aorta with a moving-core transformer. They also found that topical application of norepinephrine to the arterial wall produced decreased distensibility. Patel et al (1961) measured circumferential and longitudinal strains in vivo with calibrated electrical caliper sewed to the outer surface of the aorta. 
These authors found that addition of norepinephrine decreased circumferential extensibility with little or no apparent change in longitudinal extensibility. However, the longitudinal extensibility is so small under control conditions that small changes might easily go undetected. Finally, to complete all the possible effects of norepinephrine in vivo, Remington (1962) reported electrical and drug stimulation of vascular muscle, by and large, had no effect at all on arterial distensibility.

In some of Roy's pressure-volume studies it was noted that the greatest distensibility occurred at moderate extensions, corresponding to about $70 \mathrm{~mm} \mathrm{Hg}$ pressure. MacWilliam (1902) and MacWilliam and Mackio (1908) interpreted this inextensibility found at low extensions as a manifestation of smooth muscle tone. They noted that stiffness was not seen if the vessel was heated, frozen, treated with cyanide, ammonia vapor, or sodium fluoride solution prior to extension. If treated in this way, distention curves were not sigmoid in shape; they were simply monotonic, rising steeply at first, then flattening to a gradual slope. Presumably these treatments killed the vascular muscle.

Remington et al (1945) performed putrefaction experiments on isolated arteries in vitro. The normal pressure-volume distensibility curves of aortic segments were sigmoid in shape; they were slightly distensible at moderate pressures, 
corresponding to normal physiological conditions. After 72 hours the stiff portion of the curve encountered at low pressures was abolished. Histological examination indicated that the smooth muscle cells were in the process of degeneration, while elastin and collagen appeared quite normal. Thus, from the results of procedures which eliminated the muscle, both MacWilliam (1902) and Remington came to the conclusion that low pressure stiffness results from the action of smooth muscle. Landgren (1952) microscopically measured the diameter of the cat's carotid sinus in situ and in vitro. The carotid sinus was separated from the circulation, filled with oxygenated Tyrodes solution, and stimulated with non-oscillating intrasinus pressures. Pressure-diameter curves generated by this system provided distensibility data. Treatment with epinephrine caused an observable reduction in the diameter of the carotid sinus. It also caused a decrease in distensibility below 100 $\mathrm{mm} \mathrm{Hg}$ and above $200 \mathrm{~mm} \mathrm{Hg}$ pressure. An increase in distensibility was observed between 100 and $200 \mathrm{~mm} \mathrm{Hg}$. Treatment with sodium nitrite also slightly decreased distensibility at pressures below $100 \mathrm{~mm} \mathrm{Hg}$, and slightly increased distensibility at pressures between 100 and $200 \mathrm{~mm} \mathrm{Hg}$. Although epinephrine and sodium nitrite had qualitatively similar effects on distensibility, the magnitude of these effects was two or three times greater with epinephrine than with sodium nitrite. 
In situ pressure-volume studies were performed on isolated dog aorta (Alexander, 1954a) and on innepvated loops of dog intestine (Alexander, 1954b). Treatment with epinephrine caused constriction, slightly increased distensibility with pressures up to $100 \mathrm{~mm} \mathrm{Hg}$, and markedly increased distensibility in the range of normal physiological pressures. Carotid sinus hypotension also caused contraction and increased distensibility, but carotid sinus hypertension caused dilation and decreased distensibility. Alexander observed that the offects of smooth muscle on vascular distensibility are most pronounced at low transmural pressures, and noted that it is also at low pressures that smooth muscle caused the most pronounced shortening (1953). This agrees with the early observations of MacWilliam (1902) that the effects of smooth muscle are most pronounced at low pressures. However, MacWilliam had noted that the muscle conferred decreased distensibility, not the increase observed by Landgren (1952) and Alexander (1954a, b). Leonard and Sarnoff (1957) studied the influence of drug-stimulated smooth muscle contraction on the extensibility of venous strips. Active muscle clearly decreased extensibility at all lengths of the strip. Torrance and Shwatz (1961) excised the lower portion of the aorta including the iliac and femoral arteries. These were cannulated in a few locations, and all other patent vessels were tied. The specimen was perfused in a 
sealed bath, which also served as a volume plethysmograph. Addition of norepinephrine to the bath caused a degrease in total volume of the specimen, i.e. vasoconstriction, and a decrease in distensibility.

Burton (1954) considered the problem of how smooth muscle confers decreased distensibility of the wall. One of the few studies on smooth muscle available at the time of Burton's publication was that of Winton (1926). Burton used these data to calculated an approximate elastic modulus for smooth muscle of about $1.0 \times 10^{5}$ dynes $/ \mathrm{cm}^{2}$. Reasoning that this would not significantly increase resistance to distention, Burton proceeded to construct a mechanical model which connected smooth muscle to collagen to form a mechanical amplifier. However, in order to increase the contractile force 100 times, a 100 fold increase in shortening is required, and there is no evidence that any kind of smooth muscle can contract to this extent. Fortunately, since publication of Burton's analysis, several direct measurements of smooth muscle elasticity (Bozler, 1957) and active tension (Abbott and Lowy, 1958a) have appeared. These indicate that the force that can be developed by smooth muscle is several hundred times that calculated by Burton from Winton's data.

c. Hysteresis. If a tissue or tissue specimen is subjected to a load, the tissue uill oxiend. However, when the 
load is removed, the tissue usually will not return completely to its original length. This behavior is termed hysteresis. Hysteresis is exhibited by load-induced extension and retraction of aortic rings (Remington, 1945), by pressure-volume behavior of the isolated perfused pulmonary vasculature (Sarnoff and Berglund, 1952), by pressure-diameter behavior of the carotid sinus (Landgren, 1952), and by in vivo pressure-diameter behavior of dog aorta (Lawton, 1957; Remington, 1962).

Hysteresis has usually been explained on the basis of internal viscosity, an apparent viscous effect caused by structural rearrangements within the wall (Hardung, '1953). However, experimental studies on aortic rings (Remington, 1955) indicate that the amplitude of the hysteresis is not dependent upon the rate of stretch until some critical value is excesded.! This varies between specimens, but corresponds to a pressure increase of about $1 \mathrm{~mm} \mathrm{Hg} / \mathrm{msec}$. The amplitude of the loop appears to be unrelated to the rate of pressure decrease.

Goto and Kimoto (1966) studied the tensions developed by vascular strips under cyclic stretch. Muscular vessels exhibited more hysteresis than non-muscular vessels. Stimulating the smooth muscle mainly elevated the tension-increasing limb of the hysteresis loop. Formic acid digestion of collagen decreased both the elastic modulus and the amplitude of the loop, while trypsin digestion of elastin increased the elastic modulus and maintained the normal amplitude of the loop. In further 
studies (Kimoto and Goto, 1967) an increase in the amplitude of the hysteresis loop was observed in arteries heated over $50^{\circ} \mathrm{C}$, but this was not seen in arterial strips digested with formic acid. It thus appears that muscle, during extension from low strains, and collagen, at higher strains, play a significant role in generating arterial hysteresis under dynamic conditions.

A time-dependent phenomenon related to hysteresis is after-stretching or "Nachdehung" (Hochrein and Singer, 1927). This may also be referred to as creep, i.e. an increase in strain which proceeds, over time, under a constant extending stress. A more subtle characteristic of cre日p is that the restraining force opposing the deforming force must be falling in order for creep to occur. This decline of the resisting force is also known as stress relaxation. Stress relaxation and creep are counterparts; the phenomenon that will be observed depends upon whether tension or length is being measured. Furthermore, any increase in circumference of a cylinder under constant pressure will cause an increase in stress.

Zatzman et al (1954) measured stress relaxation in arterial strips. They found that arteries which contained much muscle (umbilical) exhibited a long time course of stress relaxation, while arteries which contained relatively less muscle (carotid) exhibited a much shorter time course of stress 
relaxation. Thus, blood vessel stress-relaxation, as well as some aspects of hysteresis, correlates with the presence of smooth muscle. 


\section{CHAPTER III}

\section{MATERIALS AND METHODS}

A. Theory. The arterial wall may be represented by the curved solid body illustrated in Figure 1A, where C denotes the circumferential or tangential direction, $L$ denotes the longitudinal direction, and $R$ denotes the radial direction. The mean stress and strain in each direction are considered in the following discussion. Stresses may be determined at a moment when the vessel. is neither expanding nor retracting. Under these conditions, the forces tending to distend the vessel are counterbalanced by equal and opposite restraining forces which tend to resist distention.

1. Stress. Circumferential stress may be determined by considering a vessel in such an equilibrium states

$$
F_{C_{D}}=F_{C_{R}}
$$

where, $F_{C_{D}}=$ the distending force in the circumferential tion, and $F_{C_{R}}=$ the restraining force in the circumferential direction. Figure 18 illustrates a longitudinal section of a vessel at equilibrium. Force is equal to the produce of a stress and the area over which that stress is exerted. Thus,

$$
F_{C_{D}}=\left(P_{T}\right)\left(d_{i} \times L\right)
$$




\section{FIGURE 1}

96

VESSEL SEGMENTS ILLUSTRATING MATHEMATICAL ANALYSIS
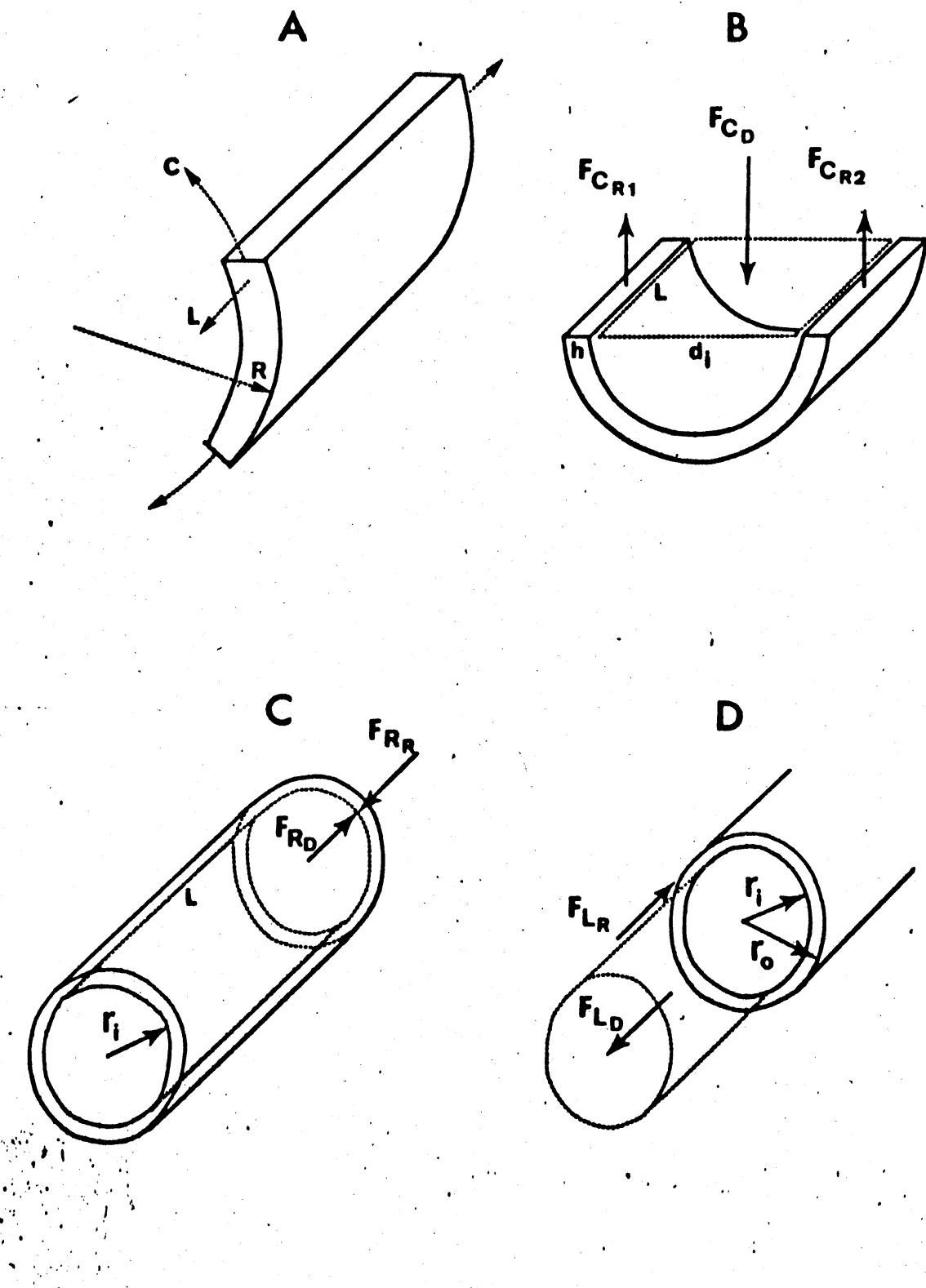

D

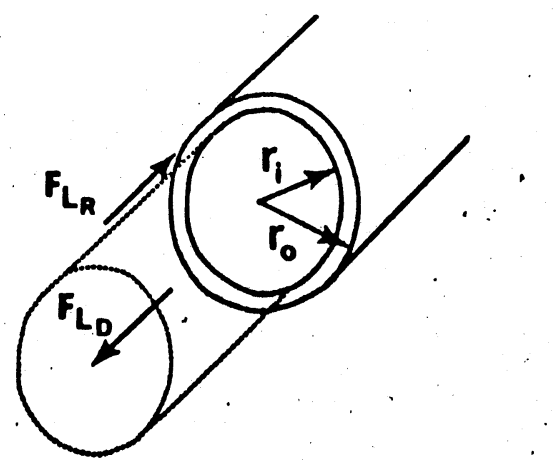


where, $\quad P_{T}=$ transmural pressure

$d_{i}=$ internal diameter

$L=$ length

Similarly,

$$
\begin{aligned}
& F_{C_{R 1}}=\left(\sigma_{C}\right)(h \times L) \\
& F_{C_{R 2}}=\left(\sigma_{C}\right)(h \times L)
\end{aligned}
$$

where, $\quad \sigma_{C}=\begin{aligned} & \text { intramural stress in a circumferential } \\ & \text { direction }\end{aligned}$

$$
\begin{aligned}
& h=\text { wall thickness } \\
& L=\text { length }
\end{aligned}
$$

The total restraining force is equal to the sum of $F_{C_{R I}}$ and - $\mathrm{F}_{\mathrm{R} 2}$. If it is assumed that the wall thickness and length of the cylindrical arterial wall are uniform, then $F_{C_{R 1}}$ and $F_{C_{R 2}}$ are equal. Therefore,

$$
F_{C_{R}}=F_{C_{R 1}}+F_{C_{R 2}}=2 \times\left(\sigma_{C}\right) \times(h \times L)
$$

Substituting equations 2 and $3 c$ for equation 1 s

$$
\begin{aligned}
& (P T)\left(d_{i} \times L\right)=\left(\sigma_{C}\right) \times 2(h \times L) \\
& \sigma_{C}=\frac{\left(P_{T}\right)\left(d_{i} \times L\right)}{2(h \times L)}=\frac{\left(P_{T}\right)\left(r_{i}\right)}{h}
\end{aligned}
$$

where $\mathbf{r}_{\dot{1}}=$ internal radius

Longitudinal stress may be calculated in a similar manner. Figure ID illustrates a cross-section of a vessel. At equilibrium, distending and restraining forces are equal:

$$
F_{L_{D}}=F_{L_{R}}
$$


where, $\quad F_{L_{D}}=$ distending force in the longitudinal direction

$F_{L_{R}}=$ restraining force in the longitudinal direction

The distending force again may be calculated as the product of the longitudinal stress and the area over which that stress is exerted.

$$
F_{L_{D}}=\left(P_{t}\right)\left(\pi r_{i}{ }^{2}\right)
$$

Similarly,

$$
F_{L_{R}}=\left(\sigma_{L}\right)\left(\pi r_{0}^{2}-\pi r_{i}^{2}\right)
$$

where, $\sigma_{. L}=$ intramural stress in a longitudinal direction $r_{0}=$ external radius

Then substituting equations 6 and 7 for equation 58

$$
\begin{aligned}
& \left(P_{T}\right)\left(\pi r_{i}^{2}\right)=\left(\sigma_{L}\right)\left(\pi r_{0}{ }^{2}=\pi r_{1}{ }^{2}\right) \\
& \sigma_{L}=\frac{P_{T} \times r_{1}^{2}}{r_{0}{ }^{2}-r_{1}{ }^{2}}
\end{aligned}
$$

Firralily, the radial stress may also be calculated by a similar procedure.. Figure IC illustrates a vessel segment and the forces involved. At equilibrium

$$
F_{R_{D}}=F_{R_{R}}
$$

where, $\quad F_{R_{D}}=$ distending force in the radial direction $F_{R_{R}}=$ restraining force in the radial direction 
The transmural pressure decreases monotonically as one progresses across the wall from inner to outer margins. At the inner margin of the wall, the intramural stress is equal to the intralumenal pressure; at the outer margin of the wall the intramural stress is equal to zero. Therefore, the mean transmural stress may be approximated as

$$
\bar{\sigma}_{R}=P_{T}=\frac{1}{Z}\left(P_{T}\right)
$$

where, $\bar{P}_{T}=$ mean transmural pressure.

$\bar{\sigma}_{R}=$ mean intramural stress in radial direction

2. Strain. Dimensional change, by convention, is expressed as strain, 1.e. percent change in dimension. Thus,

$$
\epsilon_{c}=\frac{\Delta C}{C_{0}}=\frac{2 \pi \Delta r}{2 \pi r_{0}}=\frac{\Delta r}{r_{0}}
$$

where, $\varepsilon_{c}=$ strain in the circumferential direction

$$
\begin{aligned}
& \Delta C=\text { change in circumference } \\
& C_{0}=\text { original circumference } \\
& \Delta r=\text { change in radius } \\
& r_{0}=\text { original radius }
\end{aligned}
$$

Mean circumference or mean radius should be used to be precise. However, the fact that the arterial wall is extremely thin, and represents only about $10 \%$ of the radius at most transmural pressures, permits the calculation of strain on the basis of 
external radius. This introduces only a negligible error. Longitudinal and radial strains may be calculated in - much the same manner:

$$
\varepsilon_{L}=\frac{\Delta L}{L_{0}}
$$

where, $\varepsilon_{L}=$ longitudinal strain

$$
\begin{aligned}
& \Delta L=\text { change in length } \\
& L_{Q}=\text { original length }
\end{aligned}
$$

and,

$$
\varepsilon_{R}=\frac{\Delta h}{h_{0}}
$$

where, = strain in the radial direction

$$
\text { = change in wall thickness }
$$

= original wall thickness

3. Elasticity. Stress and strain are interdependent. The proportionality constant relating these functions is the olastic modulus:

$$
E=\frac{\sigma}{\varepsilon}
$$

where, $E=$ the elastic modulus in any direction

$\dot{\sigma}=$ the stress in that direction

$\varepsilon=$ the strain in that direction

However, $E$ calculated in this way is only an approximation of the true elastic properties of a body in a given dimension, 
because it ignores the fact that a strain in one direction induces stresses and strains in the remaining directions. Consider a more precise assessment of these relationships. The least complex case is one in which it is assumed that a body is homogeneous and isotropic. Homogenity means that the elastic behavior in a given direction is uniform at every point in the body. For example, the elastic modulus in the longitudinal direction would be the same, no matter where along the length of the intact vessel the cross-section shown in Figure IC is taken. Isotropy means that the elastic moduli are equal in all directions, $i . e$. that the elastic moduli in the circumferential, longitudinal, and radial directions are identical. It was pointed out above that a strain in one direction will produce stresses and strains in other directions as well. An expression relating these interactions is Poisson's ratio. Poisson's ratio is defined as the ratio of percent contraction in one direction to percent extension in a normal direction. If this is applied to the curved body shown in Figure $1 A$, it might be said that Poisson's ratio is the ratio of radial or longitudinal contraction to circumferential elongation. If the material is isovolumetric, then Poisson's ratio will be 0.5 .

It is possible to calculate idealized, unidirectional stresses for the arterial segments illustrated in Figure 1. 
Comparison between a pure circumferential stress (equation 4b) and a pure radial stress (equation 10) reveals that the former is about 10 times the latter, at most transmural pressures. This is because the ratio of radius to wall thickness is about 10 to 1 (Hurthle, 1920; Bergel, 1959). Neglecting radial stress thus introduces only a minor error, and greatly simplifies analysis. Consider, then, the two-dimensional body illustrated in Figure 2A. This is assumed to be homogeneous and isotropic, and freely distensible. When this body is subjected to a pure stress in the circumferential direction, a circumferential strain will result:

$$
\varepsilon_{C}=\frac{\sigma_{C}}{E}
$$

where, $\varepsilon_{C}=$ strain in a circumferential direction

$$
\begin{aligned}
\sigma_{C} & =\text { stress in a circumferential direction } \\
E & =\text { elastic modulus }
\end{aligned}
$$

If the body is isovolumetric, the circumferential strain will' produce a simultaneous negative strain in the longitudinal direction. These interactions are illustrated in Figure 28, and may be expressed:

$$
-\varepsilon_{L}=\nu \varepsilon_{C}=v_{E}{ }^{\sigma} C
$$


CURVED, 2-DIMENSIONAL SHEETS ILLUSTRATING

CIRCUMFERENTIAL-LONGITUDINAL STRAINS

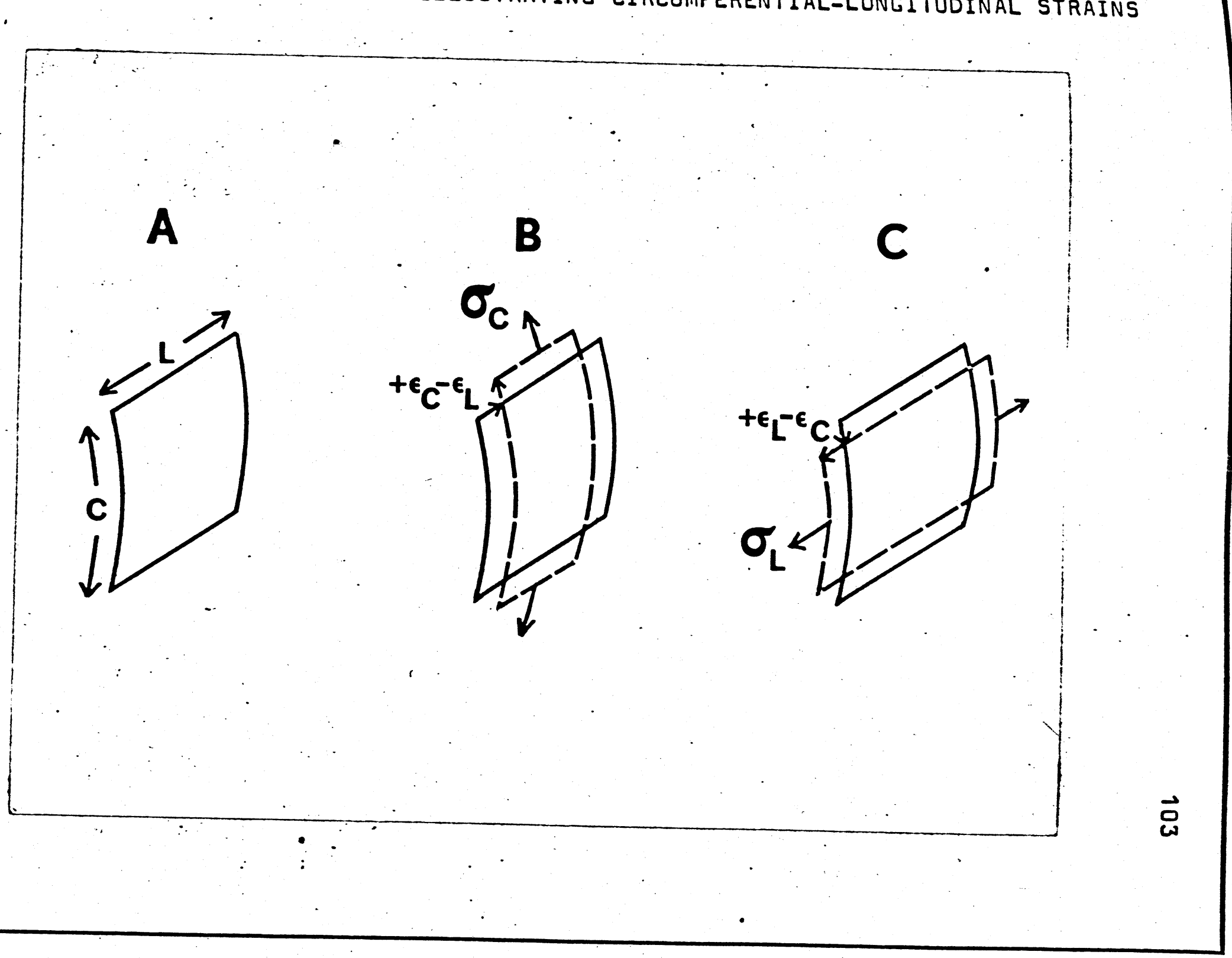


or,

$$
\varepsilon_{L}=-v \varepsilon_{C}=-v \frac{{ }^{\sigma} C}{E}
$$

where,

$\varepsilon=$ strain, the direction indicated by the subscript

$\sigma=$ stress, the direction indicated by the subscript

$\nu=$ Poisson's ratio, assumed to be 0.5 for an isovolumetric body

$E=$ elastic modulus

If, instead, the same body is subjected to a pure stress in the longitudinal direction, a longltudinal strain will result as follows:

$$
\therefore \varepsilon_{L}=\frac{\sigma_{L}}{E}
$$

where, $\varepsilon_{L}=$ strain in the longitudinal direction

$\sigma_{L}=$ stress in the longitudinal direction

$$
E \text { = elastic modulus. }
$$

These will cause a simultaneous negative strain in the circumferential direction. These interactions are illustrated in Figure 2C, and may be expressed as follows 8

$$
-\varepsilon_{C}=\nu \varepsilon_{L}=\nu \frac{\sigma_{L}}{E}
$$

or,

$$
\varepsilon_{C}=-v \varepsilon_{L}=-v \frac{\sigma_{L}}{E}
$$


where, $\varepsilon=$ strain, the direction indicated by the subscript

$\sigma=$ stress, the direction indicated by the subscript

$\nu=$ Poisson's ratio, assumed to 0.5 for an isovolumetric body

$E=$ olastic modulus

In reality, however, it is impossible to exert a pure, unidirectional stress in a cylinder distended by transmural pressure both circumferential and longitudinal stress will be produced. In order to consider the multiple interactions which result in the body shown in Figure $2 A$, both circumferential and longitudinal stresses, and their simultaneous interactions must be determined. Thus, the net circumferential strain ( $\varepsilon_{\varepsilon}{ }_{C}$ ) resulting from both circumferential and.longitudinal stresses may be determined by finding the sum of equations 15 and 18b. This sum is:

$$
\Sigma \varepsilon_{C}=\frac{{ }_{C}}{E}-v_{E}{ }_{E}
$$

Similarly, the net longitudinal strain $\left(\Sigma \varepsilon_{L}\right)$ resulting from simultaneous longitudinal and circumferential stresses may be determined by finding the sum of equations 17 and $16 \mathrm{~b}$.

$$
\Sigma_{\varepsilon}=\frac{\sigma_{L}}{E}-\nu_{E}{ }^{\sigma_{C}}
$$


Equations 19 and 20 may then be combined to give the net circumferential and longitudinal strains resulting both/from the direct effect of the stresses, and from the indirect effect of the strains that are produced by these stresses. The algebraic operation of addition of equations 19 and 20 may be simplified by multiplying both sides of equation 20 by $M$ before adding it to equation 19.

$$
\begin{aligned}
& \nu \Sigma \varepsilon_{L}=\nu \frac{\sigma_{L}}{E}-v^{2} \frac{\sigma_{C}}{E} \\
& v \varepsilon_{C}=\frac{\sigma_{C}}{E}-\nu_{E}^{\sigma_{L}} \\
& \Sigma \varepsilon_{C}+\psi \xi_{L} \varepsilon_{L}=\frac{{ }^{\sigma} C}{E}-v^{2} \frac{{ }^{\sigma} C}{E}
\end{aligned}
$$

If this equation is applied to a thin-walled cylinder in which the ends are fixed, the longitudinal strain, $\varepsilon_{L}$, is zero. The net $v \varepsilon_{L}$ term is thus zero, and the remaining equation may be solved for the elastic modulus.

$$
E=\frac{\sigma_{C}}{\varepsilon_{C}}\left(1-v^{2}\right)
$$

In an isotropic body the elastic modulus in all directions are equal. 
4. Influence of Alinearity. Equation 22 is equivalent to the expression used by Bergel (1961) to determine the circumferential elastic modulus in fixed-length arteries. Implicit in the use of this expression are the assumptions that the arterial wall is homogeneous and isotropic. The first assumption may be correct, but the definite orientation of smooth muscle cells within the wall suggest's that the arterial wall is not isotropic. However, even if both assumptions were perfectly valid, the marked alinearity of the arterial wall distensibility curves greatly complicates precise assessment. It is demonstrable that at any given transmural pressure the circumferential stress is about ten times the radial stress, and about two times the longitudinal stress. A strain in one rection produces a strain in other directions as well, but oven if the wall were absolutely isotropic, non-uniform behavior rould still result because of the combined effects of alinearity and differential stresses at a given transmural pressure. The disparity between the stresses exerted in the different directions means that at any given transmural pressure the strains oxhibited in each direction will also be different. The interaction of these stresses depends upon where each of the individual components falls on its respective alinear stress-strain arve. For example, an increment in stress in the circumferintial direction, when the circumferential strain is located 
the inextensible portion of its elasticity curve, will confer apparent inextensibility to the radial direction, even if the edial strain is simultaneously located on a relatively extensi18 portion of its respective elasticity curve. Similarly, nder these conditions, the radial strain will confer an apparant high extensibility to the circumferential direction, even shough the circumferential strain is actually on a relatively inextensible portion of its respective elasticity curve. This interdependency thus dictates that even an expression as simple a equation 22 must be used with reservation, especially with espect to the apparent precision that its use implies. A more omprehensive three-dimensional expression is required which incorporates corrections for anisotropy and alinearity. In der to obtain such corrections, data are required concerning the elastic properties in each direction. These data should obtained under experimental conditions which result in as Ittle interaction as possible. Measurement of circumferential, longitudinal and radial properties in flattened, unrestricted heots, such as shown in Figure 2, might be such an approach. itil the expanded, corrected expression is available the use if some approximation of elasticity is all that is possible. quation 22 is a reasonable estimate.

$5 \therefore$ Incremental Elastic Modulus. Equation 22 is a pasonable approximation of the elastic modulus, but only if . 
The stress-strain is constant, or very gradual in its change. In a rapidly changling, alinear curve the stress-strain ratio describes a straight line which joins the origin to some final point on the curve without coinciding with the actual curve. such a measurement is termed a "secant" modulus because it describes a straight line which cuts across the actual curve. Since the object is to measure the incremental stress-strain ratio of the actual curve, a more exact approach is to measure the slope of the actual stress-strain curve at many points along the curve. This may be termed a "tangent" or "incremental" elastic modulus (Krafka, 1939). When the stress-strain curve is linear, the secant and tangent moduli are identical; when the stress-strain curve is curvilinear, the tangent modulus is a more useful estimate. Thus, the incremental circumferential elastic modulus is given by the following expressions

$$
E_{\text {inc }}=\frac{\Delta \sigma_{C}}{\Delta \varepsilon_{C}} \cdot\left(1-v^{2}\right)
$$

B. Apparatus and Techniques. Apparatus was developed to maintain isolated arteries at in situ length in a physiological salt solution, and to measure external radius, internal radius, and wall thickness at various transmural pressures. Two lucite chambers were constructed; a large, thick-walled, 
outer chamber to serve as water jacket, and a smaller, thinwalled inner chamber to serve as a tissue bath (Figure 3 ). Water was circulated through the outer chamber, fed by a Sargent model 84880 thermostatically-controlled tissue bath heater. Warmed water was delivered by an inlet port at the lowest level of the water jacket, and was drained by an outlet port located at the highest level of the water jacket. In this way an effective circulation was maintained and the coolest water, nearest the surface, was the first to be renewed. Black ink injected into the inlet port revealed relatively stagnant regions only in the corners of the water jacket. This system maintained the temperature of the tissue bath to within $0.5 \mathrm{C}^{0}$. The temperature of the tissue bath, at the level of the arterial segment was constantly monitored with a Yellow Springs model 8443 surface thermistor and model 43-TA telethermometer. An additional heated lucite chamber (not shown in any of the figures) was used to preheat solutions destined for the tissue bath, before introducing them into that chamber. The tissue bath was constructed of $1 / 8$ inch thick lucite with a stainless steel floor to facilitate heat exchange with the water of the water jacket. The tissue bath had a $100 \mathrm{ml}$ capacity. Two upright lucite blocks were set $5.3 \mathrm{~cm}$ apart in the tissue bath chamber, and one hole was drilled in each upright on a common axis. Short segments of stainless steel tubing were pressed 
Thendow

COMPRESSED GAS

$\gamma^{2}$
PRESSURE REGULATOR

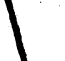


through these holes and fixed permanently with epoxy. Short segments of polyethylene PE 240 cannula tubing were forced onto the stainless steel tubes on opposing faces of the upright blocks for mounting arterial segments. Long segments of cannula were pressed onto the stainless steel tubes on the outer faces of the uprights. One of these long segments of polyethylene tubing was connected to a system of compressed gas, controlled by a Conoflow H-10XT-H pressure regulator. The gas used was $100 \%$ oxygen, except in the few cases specified where $100 \%$ nitrogen was used. The other long segment of polyethylene catheter was connected to a Statham model P23Dc pressure transducer.

A second tissue bath was constructed which was identical to the first one, except that it contained only one upright block. A plate of $1 / 2$ inch thick lucite was mounted on the movable sensing arm of a Grass model FT 10 force transducer (Figure 4). This lucite plate contained a longitudinally oriented hole through which a polyethylene PE 240 catheter was pressed. A nylon locking screw held the catheter firmly in place. One end of the catheter protruded beyond the lucite plate about $1 / 2$ inch, while the other end extended out to easily reach the pressure transducer. The force transducer was mounted on a ringstand so, that the portion of the catheter protruding from the lucite plate faced the single upright block In the tissue bath. This system provided a method for mounting 
APPARATUS USED TO MEASURE LONGITUDINAL FORCE

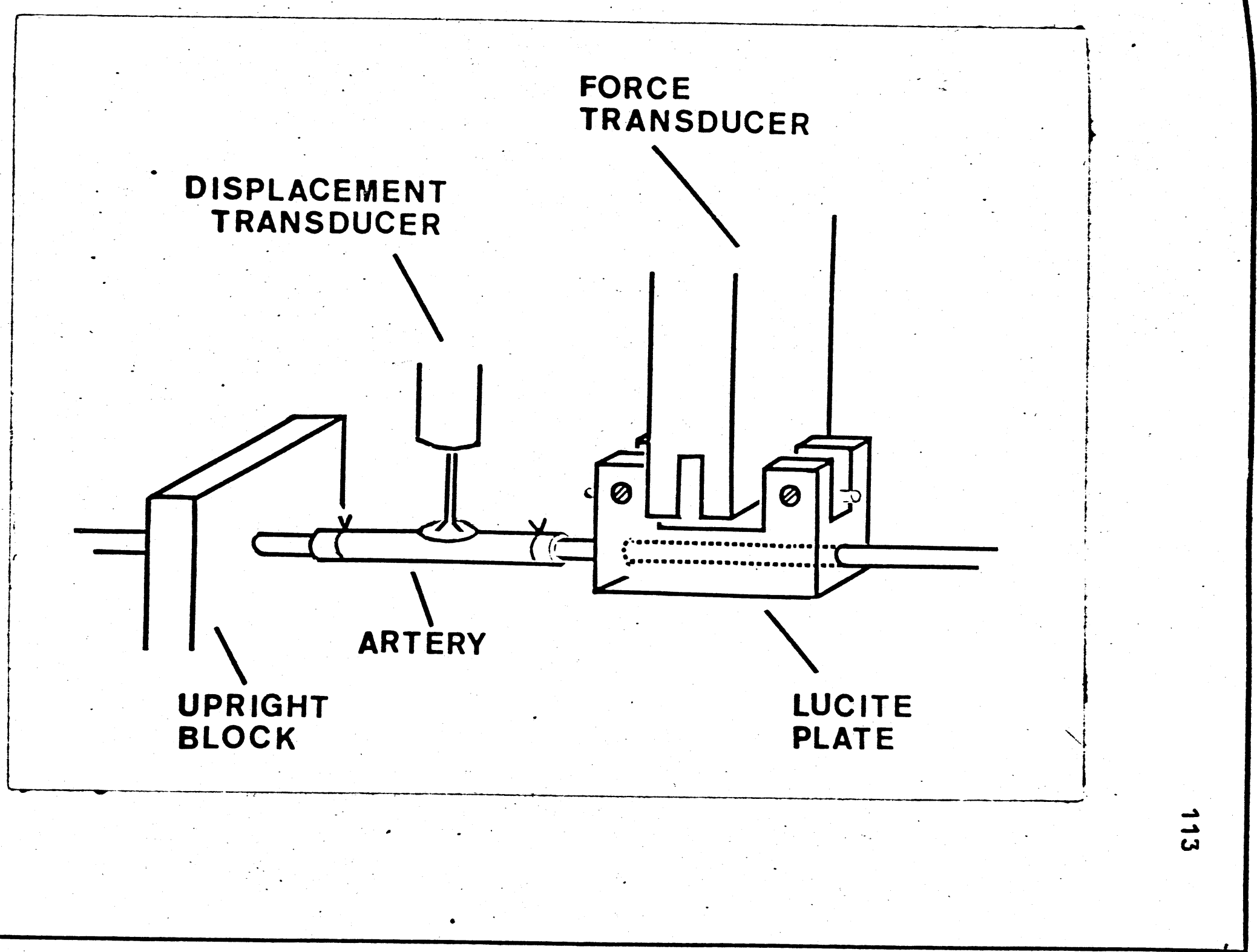


a blood vessel in a way that was identical to that employed in the tissue bath with the two-fixed uprights, but/also permitted the recording of longitudinal forces.

Excised arteries were mounted in either of the two tissue baths and were immersed under about $1 \mathrm{~cm}$ of fluid. The small pressure exerted by a column of fluid of this height was neglected, and transmural pressure was equated with the intralumenal pressure recorded by the Statham pressure transducer, except for a slight correction to be noted below.

External radius was continuously and directly measured with a linear displacement transducer (Figure 5). This consisted of a Robinson-Halpern differential transformer coupled to a Lufkin model 613 flat rod micrometer depth gauge. The flat rod on this instrument was non-rotating, and merely extended more as the micrometer was turned in a clockwise direction. A block of nylon rod was ground into a mushroom shape, and the: stem of the mushroom was threaded into the hollow steel core of the displacement transformer to increase the effective length of the core. The total core assembly, consisting of steel rod and mushroom, weighed .635 grams in air. When submerged to the depth employed during experimental procedures, the umbrella portion of the mushroom displaced a volume of fluid sufficient to reduce the effective weight of the core-mushroom assembly to .610 grams. The area of contact between the flat surface of the 
FIGURE 5

115

DISPLACEMENT TRANSDUCER

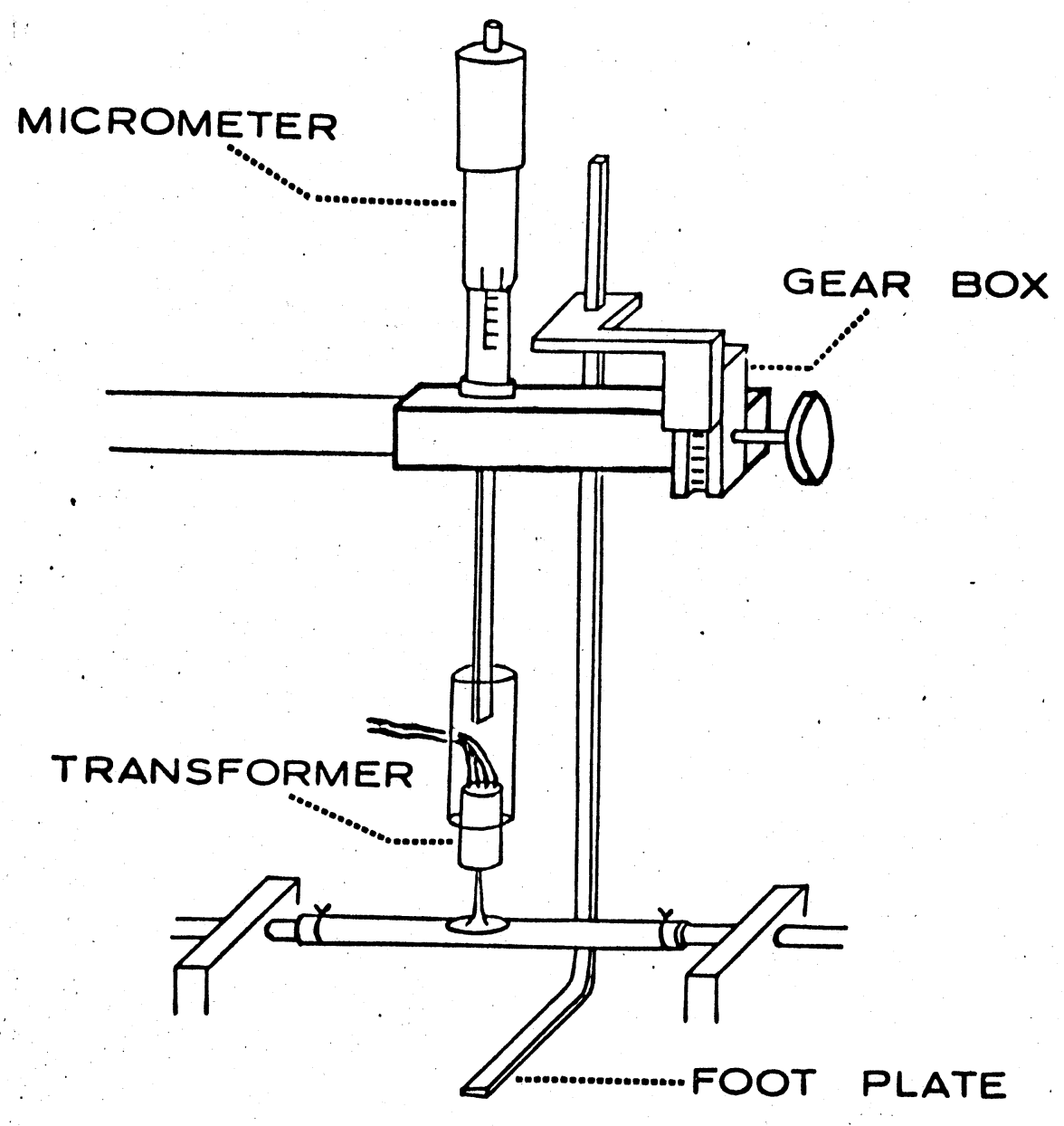


mushroom and the artery was determined by inflating a vessel mounted in the empty tissue bath with a number of transmural pressures. The vessel was wrapped with a strip of scotch tape with the adhesive surface outward, and the core-mushroom assembly was permitted to rest against the tape under the influence of.gravity: Inspection of the adhesive surface of the tape indicated that the area of contact between the mushroom and the tape was approximately $.10 \mathrm{~cm}^{2}$ over most of the pressure range. The weight of the transducer core assembly thus decreased transmural pressure by about $5 \mathrm{~mm} \mathrm{Hg}$. When arteries were mounted in the tissue bath they were immersed under about $1 \mathrm{~cm}$ of KrebsRinger solution. The small pressure exerted by a column of fluid of this height was neglected, and transmural pressure was lequated with the intralumenal pressure recorded by the Statham pressure transducer, less the $5 \mathrm{~mm} \mathrm{Hg}$ exerted by the weight of the displacement transducer core assembly.

An exciting voltage ( 4 volts, $2300 \mathrm{cps}$ ) was supplied by a Sanborn model 64-500b carrier amplifier to the primary windings of the displacement transducer coil. The Sanborn unit was also used to amplify the output of the secondary windings of the displacement transformer. The output from the Sanborn amplifier was fed into an amplifier of a Crass model 7 Polygraph. The penwriting oscillographs of the Polygraph were used to record radius, pressure and force measurements. 
The linearity of the displacement transducer was tested by permitting the mushroom to rest on the foot plate while the micrometer was turned clockwise to advance the coil downward around the core in .010 inch steps. The Polygraph pen deflection was recorded at each step. After the entire channel had been traversed, the micrometer was turned in a counterclockwise direction raising the coil from around the core in.010 inch steps. The Polygraph pen deflection was again noted. The Sanborn and Polygraph amplifiers were adjusted to give a $1.0 \mathrm{~cm}$ pen deflection for every .010 inch core-to-coil displacement. Thus, $1 \mathrm{~mm}$ channel deflection corresponded to .010 inch actual mechanical deflection. The results of one such test are illustrated in Figure 6. The points fall quite close to the line of linearity. The difference between the points obtained on ascending and descending sequences reflects the backlash inherent in the spring-loaded, non-rotating blade micrometer. However, the backlash did not enter into experimental measurements because all vital measurements were made by turning the micrometer in one direction only. According to Robinson-Halpern, the manufacturer of the transformer used in the displacement transducer, this instrument has a linear range of \pm .075 inches. However, in order to achleve adequate sensitivity for experimental applications the transformer was used only \pm .025 inches. When a vessel changed radius more 
FIGURE 6

LINEARITY OF DISPLACEMENT TRANSDUCER

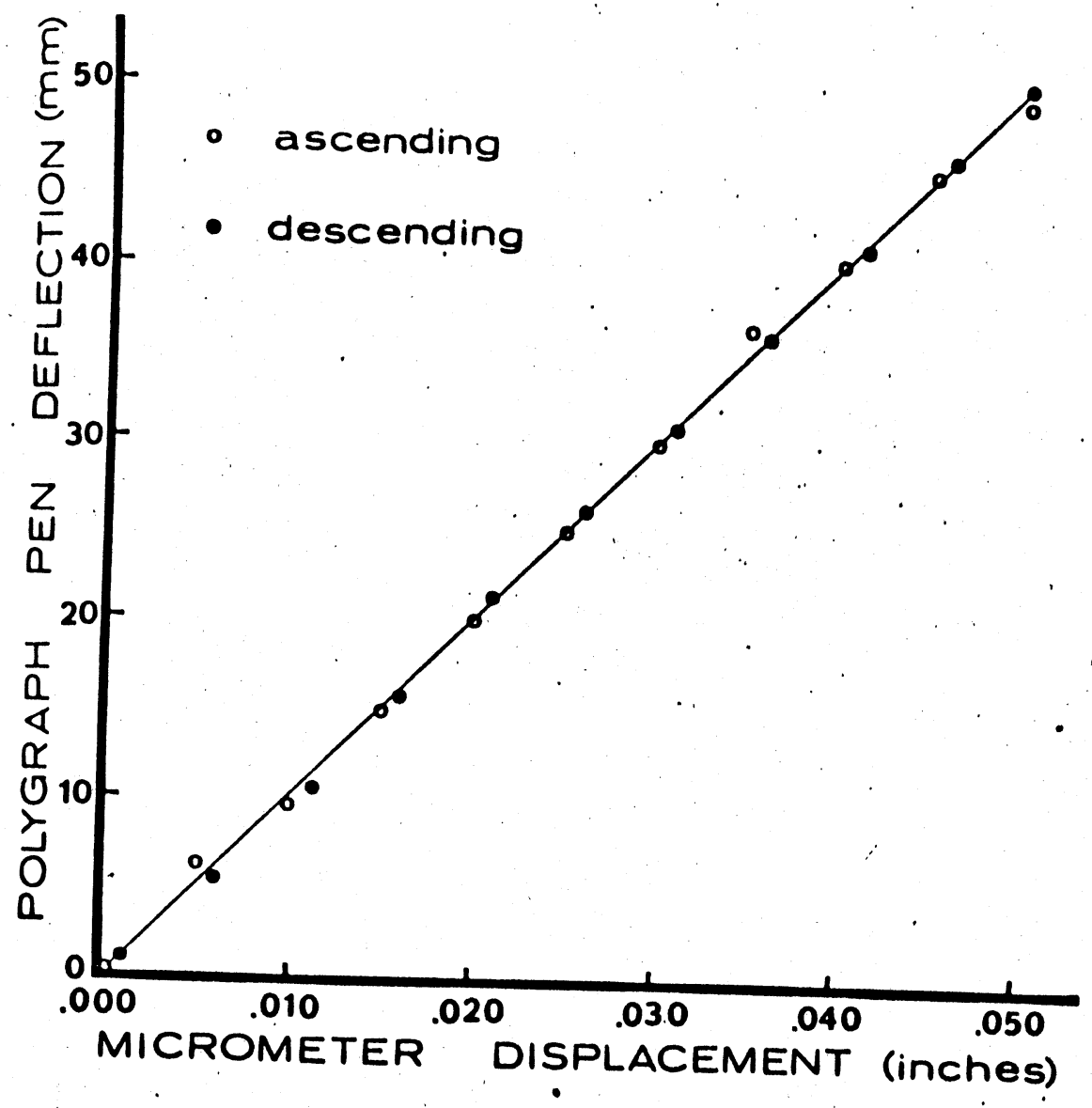


than \pm .025 inches the micrometer was turned to bring the Poly- graph pen to a more central region of the recording channel. This was done at a time when a vessel was exhibiting a steady radius. The adjustment was indicated as a shift of a given vessel size from one location to another location on the recording channel. This amounted to a shift in baseline, and permitted the use of a high sensitivity and still kept the measuring apparatus in the linear portion of the range of both the displacement transducer transformer and the Polygraph recording channel.

Although the displacement transducer was used for measuring vessels under steady state conditions, the initial change in dimension produced by a pressure change occurred at a fairly rapid rate. In order to test the dynamic response of the instrument the nylon mushroom was removed from the steel core of the transformer, and a wooden applicator stick was threaded into the core in its place. The other end of the applicator stick was threaded into the mechanical piston of a Beta oscillating pump (Yanof et al, 1963) which produced virtually perfect sinusoidal oscillations up to $90 \mathrm{cps}$. The output from the displacement transducer was recorded on the Polygraph using a paper speed of $100 \mathrm{~mm} / \mathrm{sec}$. The Beta pump was run at various speeds, for a period of about 10 sec at each frequency. The exact frequency was determined by measuring. the 
frequency of oscillations of the displacement transducer on the Polygraph record. Figure 7 illustrates the results of such a test. At frequencies up to $40 \mathrm{cps}$ the response amplitude fell at a rate of $3 \% / 10 \mathrm{cps}$. This gradual decrease in response amplitude continued until $40 \mathrm{cps}$ was reached. Above this frequency of oscillation the response amplitude fell steeply. According to McDonald (1960) and E. Attinger (personal communication), $95 \%$ or more of the variance of the pressure pulse wave can be represented by the first five fourier.harmonics. This corresponds to a maximum of 8 cps in humans, and about $15 \mathrm{cps}$ in dogs. The pressure pulse induces correlated circumferential displaceiments of the arterial wall, but the visco-elastic properties of the wall may limit mechanical displacements to frequencies lower than those encountered in the pressure pulse. The present data indicate that the displacement transducer exhibits $95 \%$ of true amplitude at a frequency of $15 \mathrm{cps}$. It was concluded, therefore, that the displacement transducer is adequate for measuring mechanical transients occurring in steady state studies. It is (well to note that the linearity and frequency response data were lobtained using the entire recording system, just as it was applied for recording experimental data.

Arteries were measured by clamping the displacement transducer to a ringstand and using the following procedure. The foot plate was lowered onto the floor of the tissue bath until the entire displacement transducer was forced slightly 
FREQUENCY RESPONSE OF DISPLACEMENT TRANSDUCER

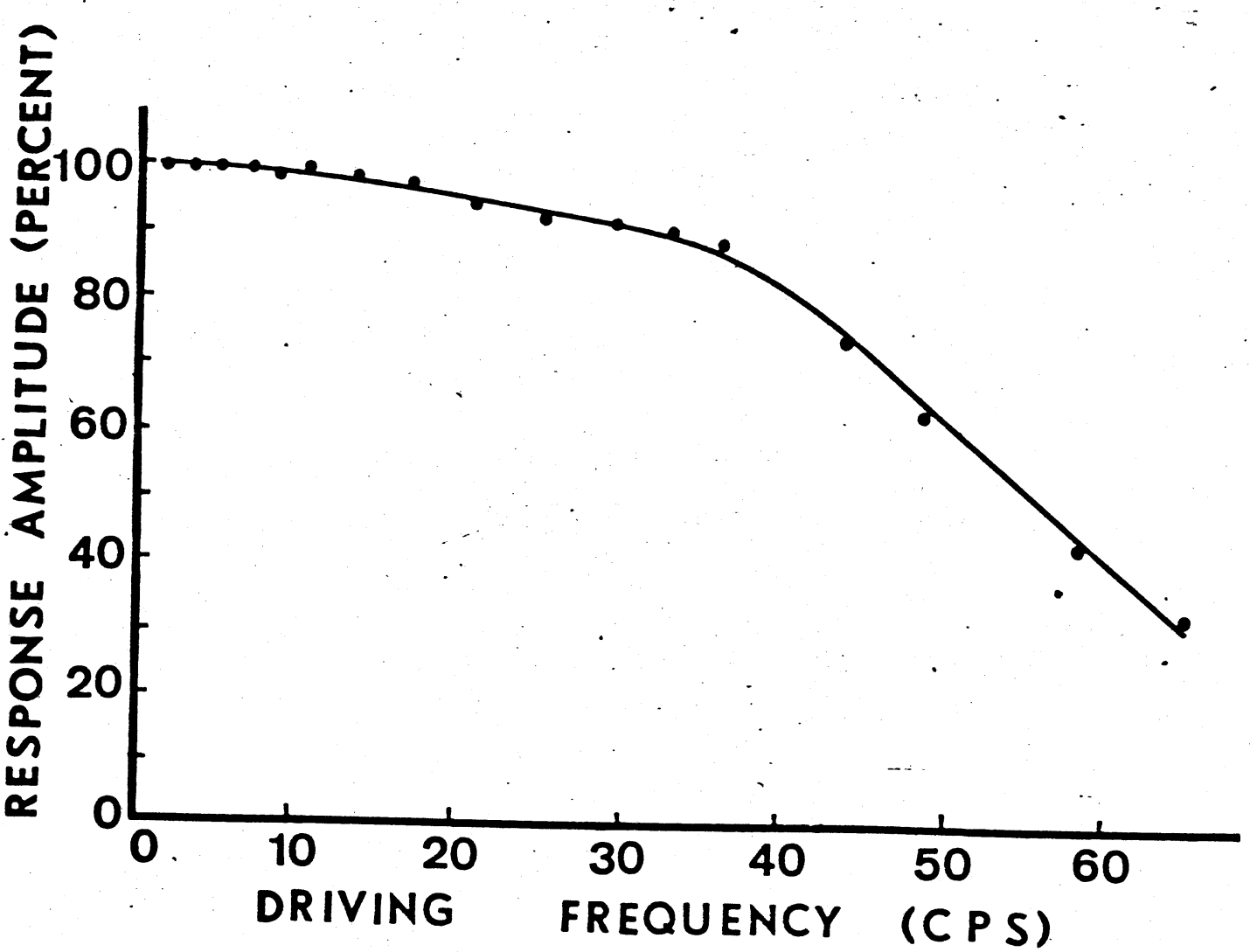


pward. This took up any slack between the ringstand and the tissue bath and mechanically coupled the tissue bath and displacement transducer. This procedure effectively removed almost all vibrations and minor mechanical disturbances from the radius recordings. Inflated vessels were visually observed to expand uniformly in a radial direction. This was because the catheters on which the vessels were mounted were set on a common axis, and also because the vessels were elongated under a longitudinal stress at all times. Observation of radiographs of these vessels also indicated that radial dilation was essentially uniform. Measurement of diameter by resting the lower surface of the artery against the foot plate was rejected because the restriction imposed by the foot Mate caused obvious visible distortion. Thus, the displace-

went transducer was used to measure only changes in external radius by recording the movements of only the upper surface of the vessel. In order to convert the changes in radius to absolute radius, the following procedure was performed. At the ind of each experiment the vessel was permitted to achieve come steady dimension under a high transmural pressure. The Hssue bath was drained and the foot plate was brought up by Use of the rack and pinion gears until the foot plate just pontacted the lower surface of the vessel. This was indicated pisual observation of changes in the light reflection of temeniscus of the Krebs-Ringer solution film remaining on 
the upper surface of the foot plate. It was also indicated by a sudden, small apparent increase in the radius of the blood vessel, as the foot plate forced the vessel upward. This was observed on the Polygraph channel recording the displacement transducer output. The micrometer was then turned clockwise, advancing the coil down around the core until the pen of the polygraph channel recording the radius was centered.precisely in the center of the channel. The micrometer reading at this point was noted. The clamp holding the displacement transducer to the ringstand was loosened and the displacement transducer moved laterally until the mushroom slid off the artery and dropped directly onto the foot plate below it. The micrometer was again turned clockwise until the pen of the Polygraph channel recording the radius was again centered precisely in the center of the channel. The micrometer at this point was again noted. The difference between the two micrometer readings was recorded as the diameter of the vessel. One-half of the value was recorded as radius and the position corresponding to this. value was noted on the Polygraph record. Knowledge of this value, and of the calibration of this channel, permitted specification of the external radius values for each of the other points on the Polygraph record. The exclusively clockwise rotation of the micrometer was adhered to in order to avoid the backlash in the instrument. 
Following completion of all pressure-radius measurements and the final diameter determination, the displacement transducer was removed and the tissue bath was lifted out of the water jacket. A dilute suspension of powdered lead oxide in saline was applied sparingly to the outer surface of the vessel, and was then washed away with clean saline. The residue which remained was so dilute that it failed to leave more than a trace of the bright yellow-white color of the lead oxide suspension. However, it did impart discernible radio-opacity. A small chip or two of dried lead oxide powder was also placed on the outer surface of the vessel in the region where the mushroom had rested, to serve as a marker. The cannula at the other end of the bath was disconnected from the gas supply and connected to a syringe containing liquid mercury. The vessel was perfused with mercury to various pressures, as indicated by the height of the mercury column in the vertical open cannula. A packet of Kodak Morelite DF-58 dental $x$-ray film was placed on a plastic block, directly beneath the vessel, and radiographs were obtained at each of the pressure levels. In some experiments a CE IICE2 dental $x$-ray machine was used with a collimating tube 16 inches long. Exposure parameters were $50 \mathrm{KVP}, 10 \mathrm{ma}, 4 / 15$ seconds. In other experiments a Ritter model $D$ dental $x$-ray machine was used with a collimating tube 16 Inches long. Exposure parameters were $54 \mathrm{KVP}, 17 \mathrm{ma}, 6$ seconds. 
$X$-rays of the vessels were measured microscopically to determine internal diameter and wall thickness., Internal diameter was measured with a Bauch and Lomb zoom lens binocular dissecting microscope. This microscope was fitted with a Cook AEl image-splitting eye-piece. The highest overall magnification was used which included the entire vessel diameter in a single field. This was usually 2 to 4 power. Wall thickness was measured with a Spencer binocular microscope using the 40 power lens with 10 power ocular containing a micrometer reticule. Typical $x$-rays are shown in Figure 8. Hypodermic needles $.025, .050$, and .072 inches in diameter, were mounted in the tis. sue bath in the same position as an artery. $X$-rays were taken of these needles, and these $x$-rays were used as standards to calibrate the image splitter and eye-piece micrometer.

A test was undertaken to compare displacement transducer measurements of an object with microscope measurements of the same object. A number of cylindrical and rod-shaped objects of uniform diameter were measured by both techniques, coded and compared by a blind method. A paper clip, aluminum and steel rods, and wooden dowels coated with the lead oxide suspension were used. Comparison between transducer and $x$-ray measurements of identical objects are presented in Figure 9. Deviation from the line of equality indicates the degree and direction of error. It is clear that there is no systematic error. These data argue 


\section{$X$-RAYS OF AN ARTERY INFLATED TO 50, 75, AND $100 \mathrm{~mm} \mathrm{Hg}$; VESSEL TREATED WITH KCN, 16.5X MAGNIFICATION.}

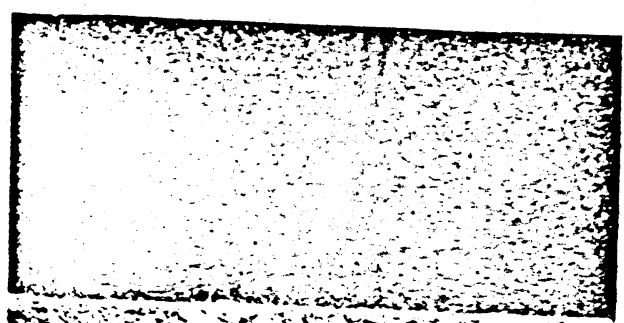

20150
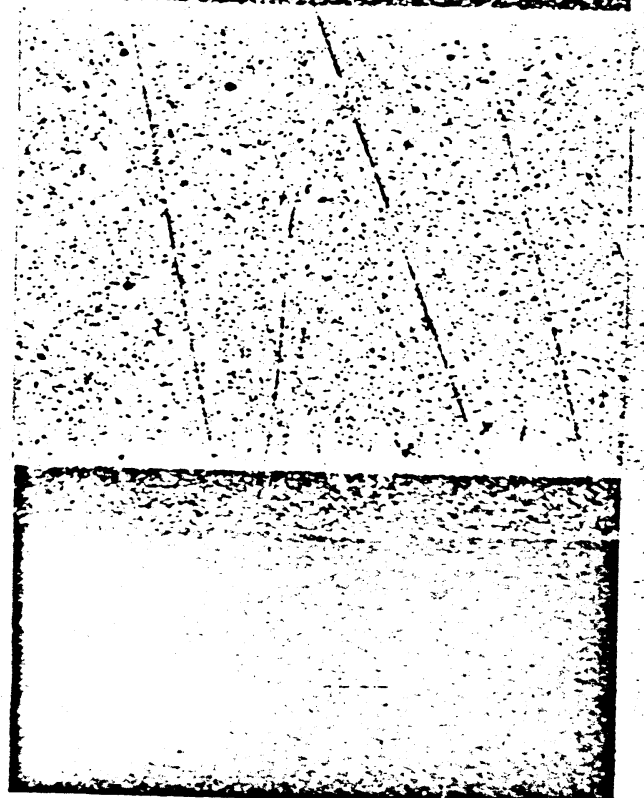

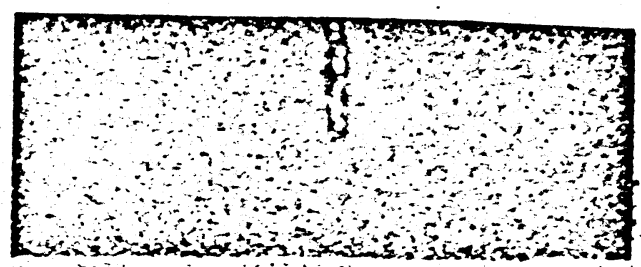

bo

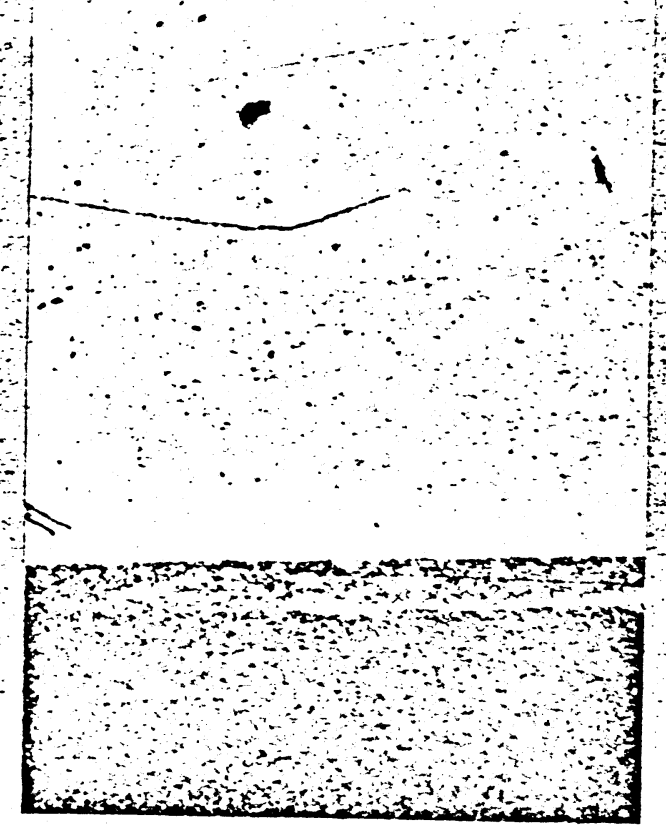

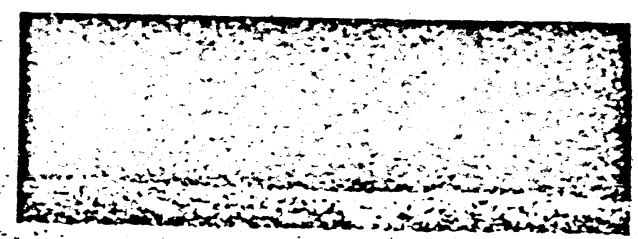
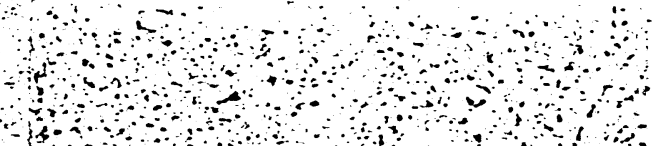<smiles></smiles>

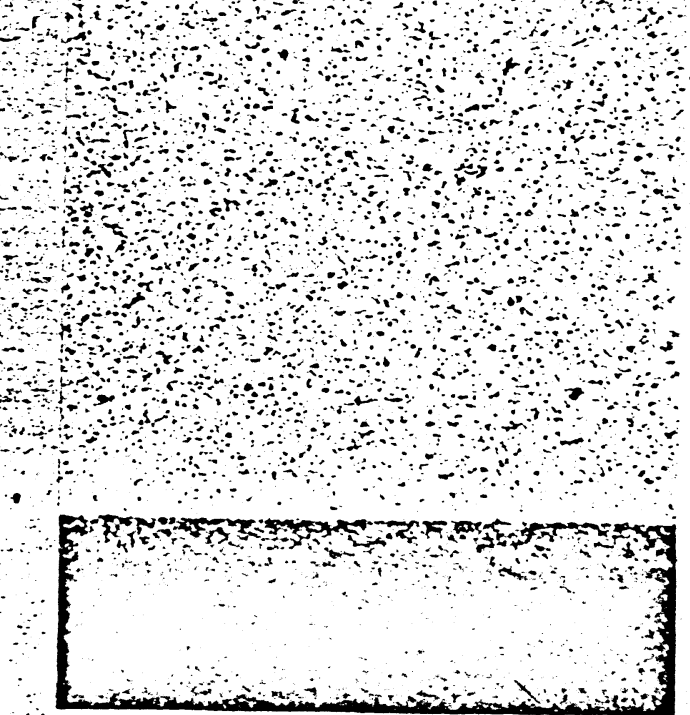




\section{X-RAYS OF THE WALL OF AN ARTERY INFLATED TO 50,75 , AND $100 \mathrm{~mm} \mathrm{Hg}$;
VESSEL TREATED WITH KCN, 10OX MAGNIFICATION.}
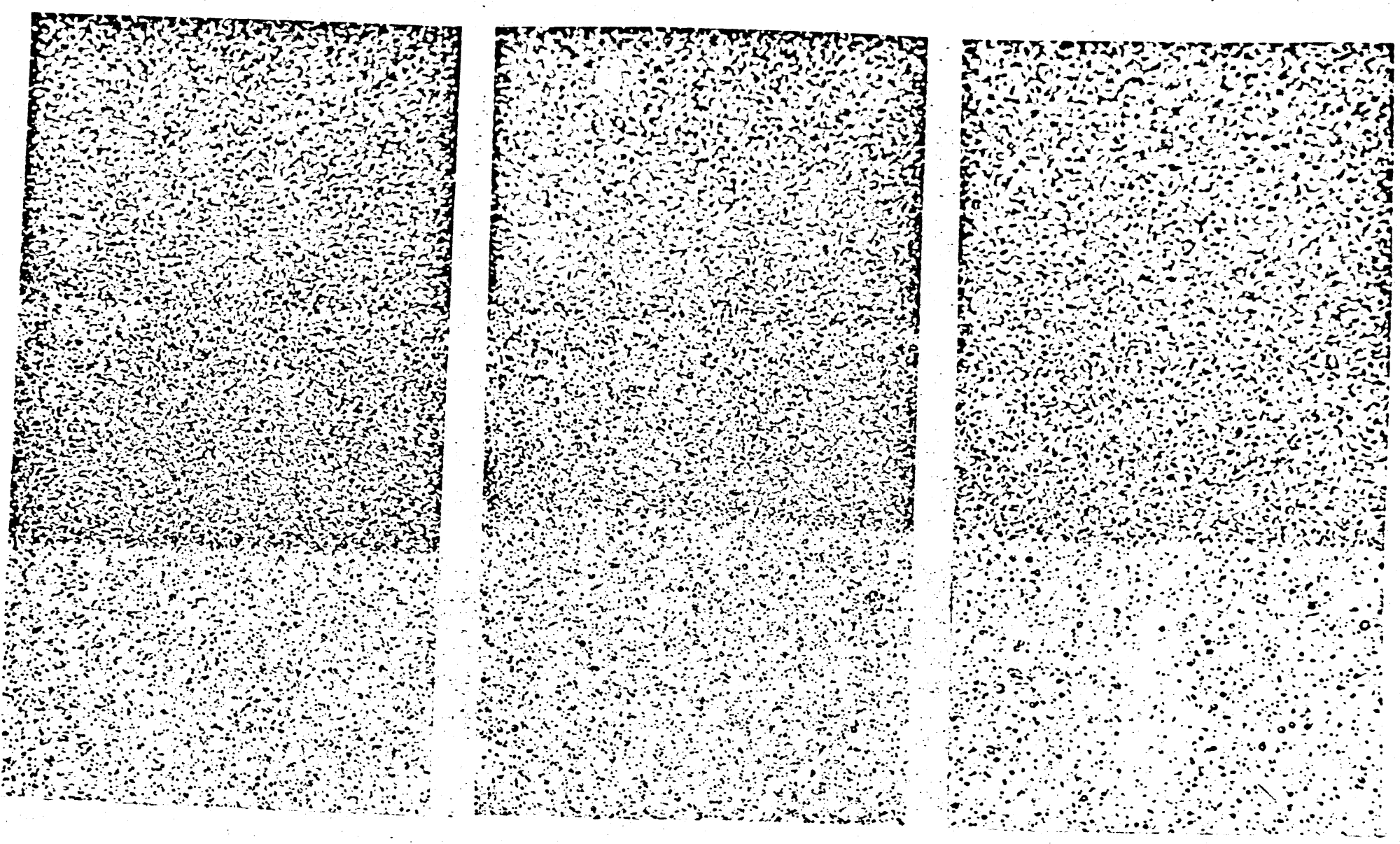


\section{FIGURE 9}

COMPARISON BETWEEN TRANSOUCER AND X-RAY MEASUREMENTS OF IDENTICAL ROD-SHAPED OBJECTS

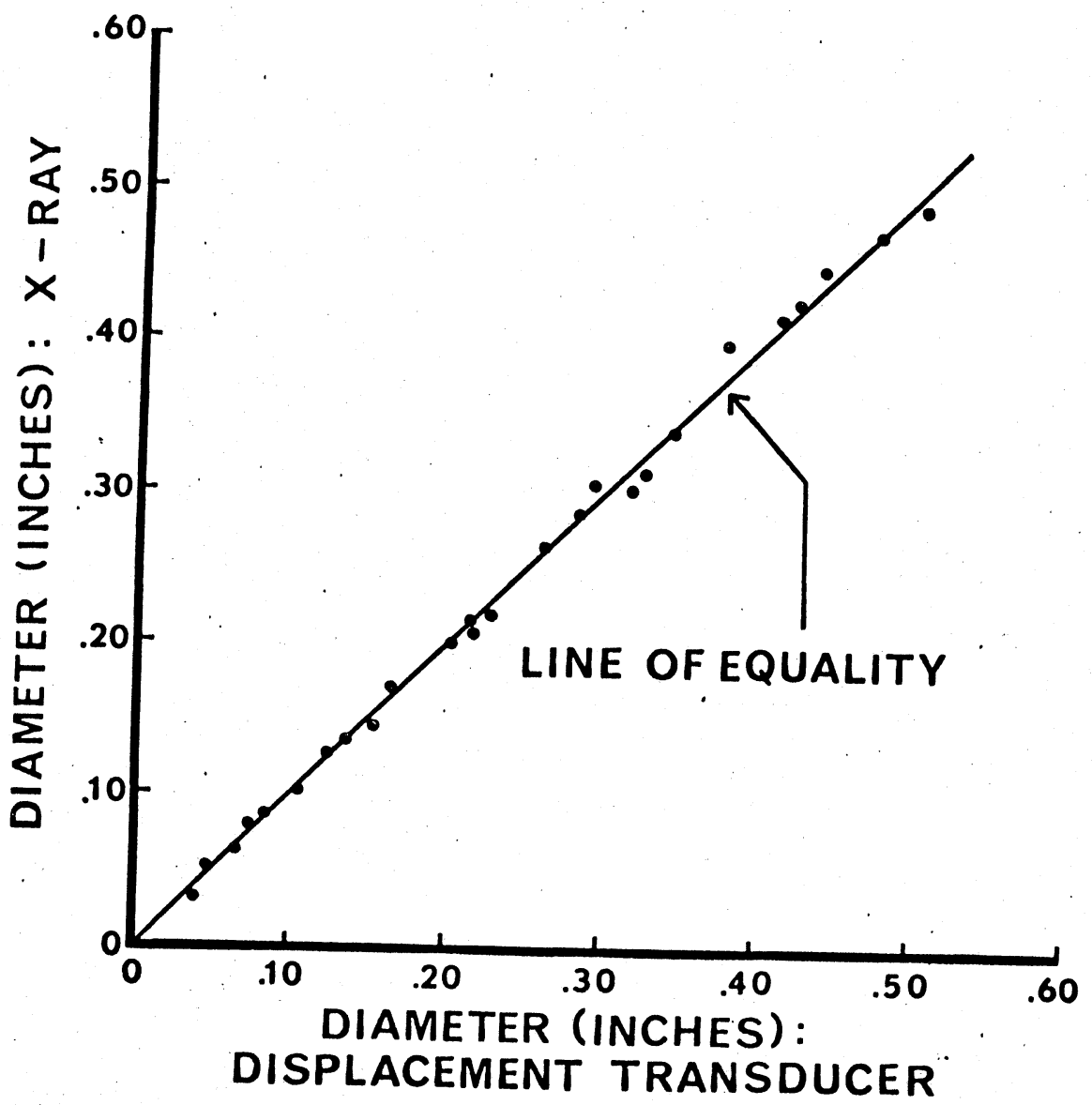


favorably for the validity of the two techniques, and the use of the two techniques in conjunction with one another. In makinh microscopic measurements on the $x$-rays only the edges are subject to real uncertainty. This uncertainty has been markedly reduced by coating the outer surface of the vessel with a radioopaque lead oxide residue. The inner surface of the wall is sharply outlined by the liquid mercury.

A number of inherently radio-opaque objects, such as aluminum and steel rods were also treated with the lead oxide suspension. The same procedure that was used on arteries was performed; the lead oxide suspension was applied to the surface of the rods, and then this was washed away. $X$-rays taken before and after lead oxide treatment failed to indicate a measurable increase in thickness due to the lead oxide. It is probable that when the lead oxide was applied to arteries, some of the radio-opaque suspension mixed with the outer layers of the extracellular water and remained there when the bulk of the surface particles were washed away. If the lead oxide was placed on metal rods in Iocalized areas and not washed away, then distinct "high spots" of slightly increased diameter could be discerned on $x$-rays of these preparations. Once washed, however, the high spote disappeared.

C. Procedure. 1. General Procedure. Mongrel dogs of both sexes; 9-12 kilograms body weight, were anesthetized 
with either $32 \mathrm{mg} / \mathrm{Kg}$ Nembutal (Abbott) intraperitoneally or $2 \mathrm{mg} / \mathrm{Kg}$ Sernylan (Parke-Davis) intramuscularly and $80 \mathrm{mg} / \mathrm{Kg}$ a-chloralose (Kuhlmann) intravenously. An incision was made in the mid-cervical region and a carotid artery was exposed. A 5.3 cm segment of the vessel was measured with calipers and the vessel was marked with a small nick in the adventitia. The artery was ligated a short distance from either side of the marked segment, and the segment was excised. It was rinsed and flushed with $25^{\circ} \mathrm{C}$ Krebs-Ringer solution. The vessel was immediately trimmed of excess connective tissue and mounted on the cannulas in the tissue bath. In mounting, the retracted artery was reelongated to the original length of $5.3 \mathrm{~cm}$. The interval from the time of excision to the time of mounting was 3 to 5 minutes. The vessel was then completely immersed in buffered Krebs-Ringer solution held at $36.5 \pm 0.5^{\circ} \mathrm{C}$. This solution was prepared according to Umbreit et al (1957). It was composed of $120 \mathrm{~mm}$ $\mathrm{NaCl}, 4.8 \mathrm{mM} \mathrm{KCl}, 2.5 \mathrm{mMCaCl}_{2}, 1.2 \mathrm{mM} \mathrm{MgSO} 4^{\cdot 7} \mathrm{H}_{2} \mathrm{O}, 15 \mathrm{~mm}$ phosphate buffer - $\mathrm{pH} 7.4$ and $11.0 \mathrm{~mm}$ dextrose. The foregoing-described procedure of excising arteries and mounting them at in situ length in a Krebs-Ringer bath was followed for all of the following experiment, except those specifically stated otherwise.

2. Wall Volume. A number of arteries were excised, Iinsed and flushed with Krebs-Ringer solution, and were mounted, 
dry, at in situ length, in the tissue bath. Each artery was subjected to the following procedure: The outer surface of the vessel was coated with a trace layer of lead oxide, along with a number of lead oxide marker chips. The lumen was perfused with liquid mercury under a variety of pressures ranging from 40 to $250 \mathrm{~mm} \mathrm{Hg}$. Perfusion pressures were begun at lowest levels and increased sequentially to highest levels. X-rays were taken of the artery at each pressure level. Internal radius and wall thickness were microscopically measured on each $x$-ray, and wall volume was calculated as follows:

$$
V=L\left(\pi r_{0}^{2}-\pi r_{i}^{2}\right)
$$

where, $\quad V=$ volume

$$
\begin{aligned}
L & =\begin{array}{l}
\text { length between the ligatures of the cannulated } \\
\text { segment }
\end{array} \\
r_{0} & =\text { external radius } \\
r_{i} & =\text { internal radius }
\end{aligned}
$$

The length of each preparation was held constant, and was therefore treated as unity.

3. Action of Norepinephrine and Potassium Cyanide. a. Dose-Response Relationships. Arteries, were excised, rinsed and mounted at in situ length in the tissue bath, immersed in Krebs-Ringer dextrose solution. Each vessel was perfused with 
100\% oxygen gas. Transmural pressures were increased in $25 \mathrm{~mm}$ Hg steps to $200 \mathrm{~mm} \mathrm{Hg}$. They were then decreased in a stepwise fashion to $75 \mathrm{~mm} \mathrm{Hg}$ while the radius of the arteries was continuously recorded with the displacement transducer. The transmural pressure was held at each step until the displacement transducer indicated a steady radius for 2 minutes or more. Equilibrium usually was achieved in 5 to 10 minutes. These steps will be referred to as "equilibrium steps". At $75 \mathrm{~mm} \mathrm{Hg}$, vessels were treated sequentially with $0.1,1.0,10.0,100.0$, and $300.0 \mu$ of norepinephrine (NEpi) (Winthrop) per $100 \mathrm{ml}$ of tissue bath fluid. The drug was administered in drops in the vicinity of the arterial segment. Each dose of the drug used was that amount necessary to bring the total concentration up to the level stipulated. For example, after the 0.1 ug dose was given and the change in radius recorded, $0.9 \mu \mathrm{g}$ was given to bring the following total dose level to $1.0 \mu \mathrm{g} / 100 \mathrm{ml}$ of bath. No attempt was made to wash out the previous dose. A fresh ampule of the drug was opened each day shortly before administration, and diluted when necessary, in isotonic saline. The total volume injected was $1 \mathrm{ml}$ for all doses.

A second group of arteries also was excised, rinsed and mounted at in situ length in the tissue bath, and immersed in Krebs-Ringer dextrose solution. They were subjected to the identical stepwise transmural pressure sequences as the NEpitreated arteries described above, but the ascending sequence 
of pressure steps was stopped at $200 \mathrm{~mm} \mathrm{Hg}$. These vessels were treated with $100 \mu \mathrm{g} / 100 \mathrm{ml}$ norepinephrine and permitted to equilibrate. The bath was then changed several times with fresh $36-37^{\circ} \mathrm{C}$ Krebs-Ringer solution which contained no dextrose. A dose of potassium cyanide $(K C N) 0.1,1.0,10.0$, or $60 \mathrm{mg} / 100$ $m l$ of bath was injected into the bath. Each dose was diluted in isotonic saline so that the total volume of each dose was $1 \mathrm{ml}$. One hour was permitted to elapse after treatment with one of these doses, and the degree of dilation produced by the cyanide was recorded. It was found, in pllot studies, that $60 \mathrm{mg} / 100 \mathrm{ml}$ of bath KCN caused a degree of dilation which could not be significantly increased by any higher additional dose of cyanide. A $60 \mathrm{mg} / 100 \mathrm{ml}$ of bath dose was therefore given after the vessel had been subjected to some lower dose for one hour. An additional hour was permitted to elapse after the dose of $60 \mathrm{ml} / 100$ $\mathrm{ml}$ of bath and the degree of dilation achieved at this time was considered to be maximum.

b. Time Course of KCN Action. A number of arteries were excised, mounted in the tissue bath at in situ length, and immersed in $36-37^{\circ} \mathrm{C}$ Krebs-Ringer dextrose solution. The arteries were inflated with $100 \%$ oxygen, while external radius was continuously recorded with the displacement transducer. The transmural pressure was increased in $25 \mathrm{~mm} \mathrm{Hg}$ equiligrium steps to 200-250 mm Hg, and then decreased to 150-200 mm Hg. A 200 $\mu g / 100 \mathrm{ml}$ dose of $N E p i$ was given. After about 15 minutes the 
bath was rinsed and refilled with fresh dextrose-free KrebsRingers solution, and a $60 \mathrm{mg} / 100 \mathrm{ml}$ dose of $\mathrm{KCN}$ was administered. The external radius of the artery was then recorded for up to 8 hours to observe the time course of KCN action. In a few experiments an additional $200 \mathrm{mg} / 100 \mathrm{ml} \mathrm{KCN}$ was given at the end of the period and an additional hour was permitted to elapse to evaluate the degree of dilation.

c. Specificity of NEpi and KCN. Another group of arteries was excised and subjected to one of a number of procedures to kill the arterial muscle. These procedures were: a) exposure to a dextrose-free, anoxic environment by inflation of the vessels with $100 \%$ nitrogen gas for 8-10 hours; b) immersion in distilled water for 10-12 hours; c) immersion in KrebsRinger solution and subjection to slow freazing for 10-12 hours; and d) immersion in Krebs-Ringer solution and subjection to heating to $500 \mathrm{C}$ for one hour, with approximately 40 additional minutes required to heat up to, and cool down from, that temperature. Those vessels immersed in distilled water and those that were frozen were subjected to these treatments prior to mounting in the tissue bath. They were immersed in fresh Krebs-Ringers solution for about eight hours before being mounted and tested. Those arteries subjected to nitrogen exposure, and those exposed to $50^{\circ} \mathrm{C}$ temperatures were subjected to those procedures while mounted in the tissue bath. Following 
its respective procedure, each artery was immersed in fresh 36-37 $\mathrm{C}$, Dextrose-free Krebs-Ringer solution and was inflated with 100\% oxygen. Transmural pressures were increased in 25 $\mathrm{mm} \mathrm{Hg}$ steps up to $250 \mathrm{~mm} \mathrm{Hg}$, and then decreased in $25 \mathrm{~mm} \mathrm{Hg}$ steps to either $175-200 \mathrm{~mm} \mathrm{Hg}$ or to $50-75 \mathrm{~mm} \mathrm{Hg}$. External radius was recorded with the displacement transducer. Each artery was treated sequentially first with $200 \mathrm{\mu} / 100 \mathrm{ml} \mathrm{NEpi}$, and then with $60 \mathrm{mg} / 100 \mathrm{ml} \mathrm{KCN}$. Adequate time was permitted to observe the diameter changes produced by these agents. d. Effect of NEpi and $K C N$ on Wall Volume. An additional group of arteries was excised and mounted in the dry tissue bath at in situ length. The outer surface of the vessel was coated with a trace layer of lead oxide, and a number of lead oxide chip markers. The bath was then filled with Krebs-Ringer dextrose solution, and the artery was inflated with $100 \%$ oxygen. The transmural pressure was increased in $25 . \mathrm{mm} \mathrm{Hg}$ equilibrium steps to $100 \mathrm{~mm} \mathrm{Hg}$. The displacement transducer was removed and $200 \mu \mathrm{g} / 100 \mathrm{ml} N \mathrm{Npi}$ was administered. After 30 minutes the bath was drained and the artery was filled with liquid mercury under a range of perfusion pressures. An x-ray was taken at each pressure. Following this procedure the tissue bath was gently ringed and refilled with fresh glucose-free Krebs-Ringer solution, and $60 \mathrm{mg} / 100 \mathrm{ml} \mathrm{KCN}$ was added to the bath. After one hour the bath was again drained and $x$-rays 
were taken over a range of liquid mercury perfusion pressures. Wall volumes were determined after both. NEpi treatment and KCN treatment for each artery.

4. Influence of Smooth Muscle on Mechanical Charac-. teristics. a. Shortening and Extending Cycles. Arteries were excised, rinsed and mounted at in situ length on cannulas protruding through the upright blocks in the tissue bath. They were immersed in Krebs-Ringer dextrose solution and inflated with $100 \%$ oxygen while external radius was continuously recorded. Transmural pressures were increased in $25 \mathrm{~mm} \mathrm{Hg}$ steps to 200-225 $\mathrm{mm} \mathrm{Hg}$, and then decreased in this stepwise fashion to $25 \mathrm{~mm} \mathrm{Hg}$, and back up to $200-250 \mathrm{~mm} \mathrm{Hg}$. In some experiments $350 \mathrm{~mm} \mathrm{Hg}$ was used for the high pressure, and in some $0 \mathrm{~mm} \mathrm{Hg}$ was used for the low pressure. At the high pressure the arteries were treated with $200 \mu \mathrm{g} / 100 \mathrm{ml} \mathrm{NEpi}$ and the vessel was permitted to contract until a new equilibrium was established. The pressure was then decreased to the low pressure in $25 \mathrm{~mm} \mathrm{Hg}$ equilibrium steps, and then increased again, in steps, to high pressure. At high pressure the tissue bath was drained, rinsed, and refilled with dextrose-free Krebs-Ringer solution. A $60 \mathrm{mg} /$ $100 \mathrm{ml}$ dose of KCN was added to the bath and the vessel was per-. mitted to remain at $200 \mathrm{~mm} \mathrm{Hg}$ or more for one hour. At the end of this period the artery was again subjected to $25 \mathrm{~mm} \mathrm{Hg}$ equilibrium pressure steps down to a low pressure, and back up to a high pressure level. In a few experiments the pressures were 
decreased to zero, and even to negative values only after KCN treatment. In some experiments all steps were oquilibrium steps in others either the shortening or distention phases were carried out in steps performed as the vessel approached, but did not yet attain full, final equilibrium. At the end of these procedures the diameter of the artery was determined at high pressure, and finally, $x$-rays were taken of each vessel.

5. Hysteretic Behavior. a. Interrupted Loops. Arteries were excised, rinsed and mounted at in situ length. The vessels were subjected to pressure cycles in pretreatment, NEpitreated and cyanide-poisoned states, just as described in the previous section. A modification of these procedures was that the distending limb was halted before reaching the maximum pressure. A sequence of descending equilibrium steps was performed from that point. In some experiments the distention limb was repeated and this was also halted before maximum pressure. to give a second shortening sequence.

b. Shortening from Multiple Origins. In order to systematically analyze the characteristics of the hysteresis loop, a number of arteries were mounted at in situ length and lere subjected to the following procedures. After completion of the pretreatment cycle the arteries were treated with $200 \mathrm{Ng} / 100$ VN NEpi at a high pressure, and the pressure was decreased in fquilibrium steps. When the vessel achieved stability at the 
low pressure, the pressure was increased in a single step to some higher, "starting" pressure. The radius associated with this starting pressure may be referred to as the "origin". A sequence of descending equilibrium steps was then carried out until the lowest pressure in the cycle was achieved. This procodure was repeated until shortening curves originating from many different starting pressures were obtained. The order of starting pressures was randomized. The same procedure was followed after one hour poisoning with cyanide at high pressure. C. Distention from Multiple Origins. An additional group of arteries was studied in a similar manner to obtain distention sequences commencing from various starting pressures. After a full pretreatment cycle, the artery was treated with $200 \mathrm{ug} / 100 \mathrm{ml} \mathrm{NEpi}$ at a high pressure. A full decrease-increase pressure cycle was performed, and the pressure was dropped in a single step to some lower "starting" pressure. A sequence of ascending equilibrium pressure steps was performed from this origin. This procedure was repeated until distention curves originating from many origins were obtained. The order of starting pressures was randomized.

6. Longitudinal Properties. Arteries were excised, rinsed and one and of the vessels was mounted on a cannula protruding through one of the upright blocks in the tissue bath. The other end of the artery was cannulated with flexible silastic 
tubing, and this cannula was secured to the lucite plate and connected to the pressure transducer. The radiug of these preparations was measured with the displacement transducer, and the longitudinal force was measured with the force transducer. The vessels were subjected to an initial stepwise distention, and this was followed by the usual pressure cycles in the pretreatment condition, after treatment with NEpi and after poisoning with cyanide. A pressure of 200-225 was used as the upper limit. and 0-25 was used as the lower limit. Upon completion of these procedures the lucite plate was removed from the cannula, and the cannula was clamped in place to maintain the appropriate length. The vessel was coated with a thin layer of lead oxide, perfused with liquid mercury and $x$-rayed over a wide range of distending pressures. At the end of the experiment the force transducer was turned so that the sensing arm could be deflected vertically, and the displacement transducer mushroom was rested against the sensing arm. The arm was deflected to the same extent observed on the Polygraph record during experimental procedures. This test indicated that the elongated artery was permitted to change less than .01 percent in length. 
CHAPTER IV

\section{RESULTS}

A. Wall Volume. Carotid arteries were perfused under pressure with liquid mercury, and the wall volumes were determined at each pressure. These data are shown in Figure 10. The mean wall volume of each artery was designated as $100 \%$ and the points contributing to that mean were then plotted against diameter. Each artery is represented by a different symbol; all the open circles indicate individual volume determinations for one artery, all the closed circles for another artery, etc. The abscissa is external diameter, expressed as the percent of each individual vessel's diameter at $50 \mathrm{~mm} \mathrm{Hg}$. The ordinate is wall volume, expressed as the percent of imean wall volume for each vessel. These data are expressed in percentage form in order to compare wall volume of arteries obtained from animals of different sizes and weights. If the wall underwent volume compression with increased distention, then the points would show a general tendency to fall from left to right. If the wall underwent a volume expansion with increased distention, then the points would show a general tendency to rise from left to right. It is clear from 
FIGURE 10

ARTERIAL WALL VOLUME

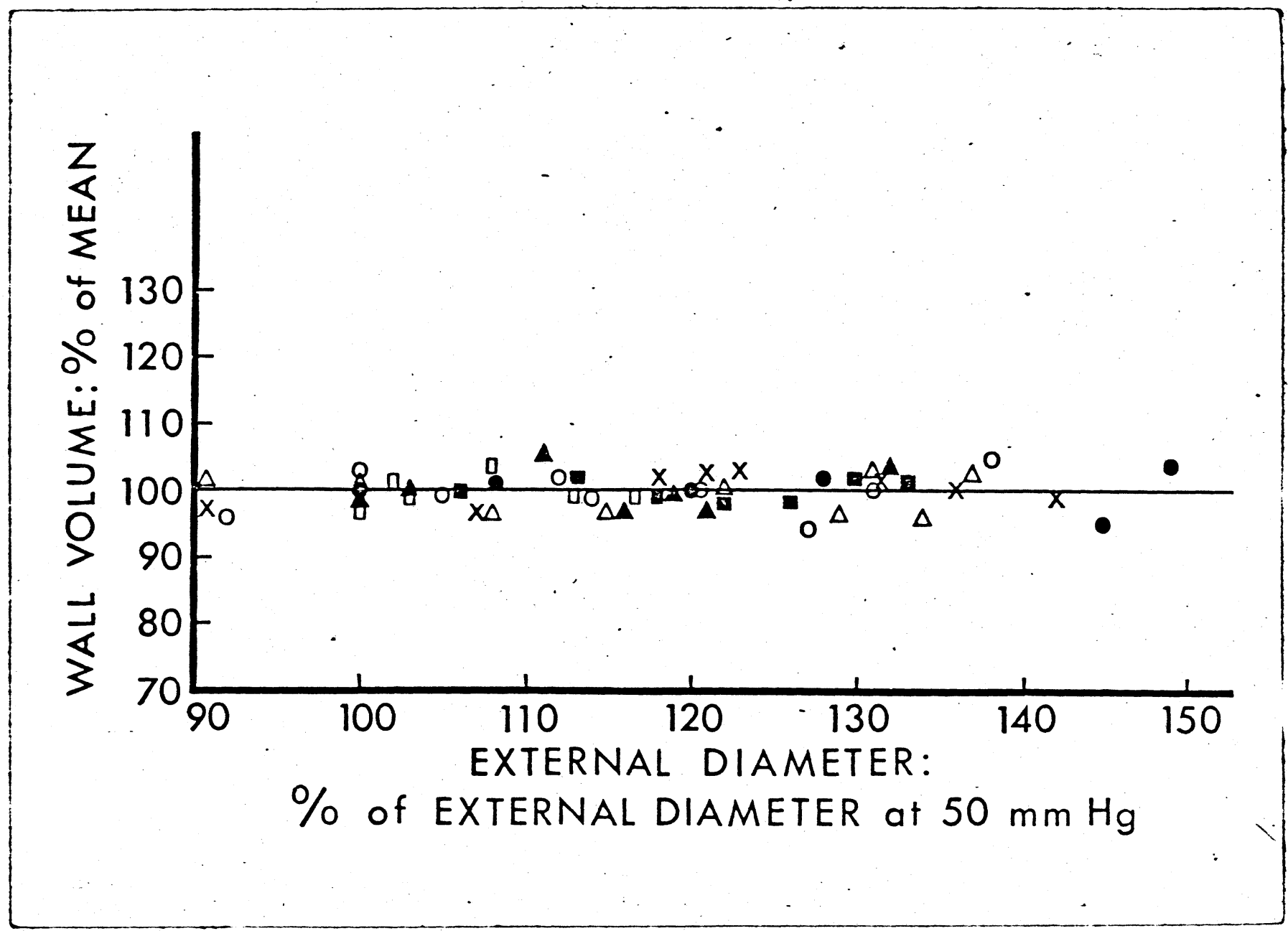

Data are for 7 arteries, each represented by a different symbol. The tendency for the points to fall randomly about the mean indicates 
Figure 10 that neither trend is observed, and it may be concluded therefore, that the arteries in this preparation exhibit isovolumetricity. This means that each change in diameter is accompanied by an appropriate, opposite change in the thickness of the wall, and the manitude of change in the thickness is dictated by the incompressibility of the material of the wall. The isovolumetricity of the arterial wall may be expressed mathematically as a Poisson's ratio of 0.5 .

B. Actions of NEpi and KCN. 1. Dose Response relationships. Figure 11 presents portions of polygraph records illustrating the response of a carotid artery, fixed at in situ length in the tissue bath, distended with $175 \mathrm{~mm} \mathrm{Hg}$ pressure, and treated with $200 \mu \mathrm{g} / 100 \mathrm{ml} \mathrm{NEpi}$ and $60 \mathrm{mg} / 100 \mathrm{ml}$ KCN. Figure $11 \mathrm{~A}$ provides an example of the prompt arterial contraction produced by $200 \mu g / 100 \mathrm{ml}$ NEpi. An additional dose of $300 \mu \mathrm{g} / 100 \mathrm{ml}$ NEpi caused no further contraction. Figure 11B provides an example of the dilation produced by $60 \mathrm{mg} / 100 \mathrm{ml} \mathrm{KCN}$. An additional $200 \mathrm{mg} / 100 \mathrm{ml}$ dose of KCN given after 90 minutes caused no further dilation.

Figure $12 \mathrm{~A}$ presents a NEpi dose-response curve. The abscissa is the dose of the drug, plotted on a logarithmic scale, and. the ordinate is the amplitude of contraction expressed in percent of the maximum observed in each individual experiment. The points indicate the mean determined for 9 
POLYGRAPH RECORDS ILLUSTRATING CONTRACTILE EFFECTS

OF NEPI, AND DILATION PRODUCED BY KCN.

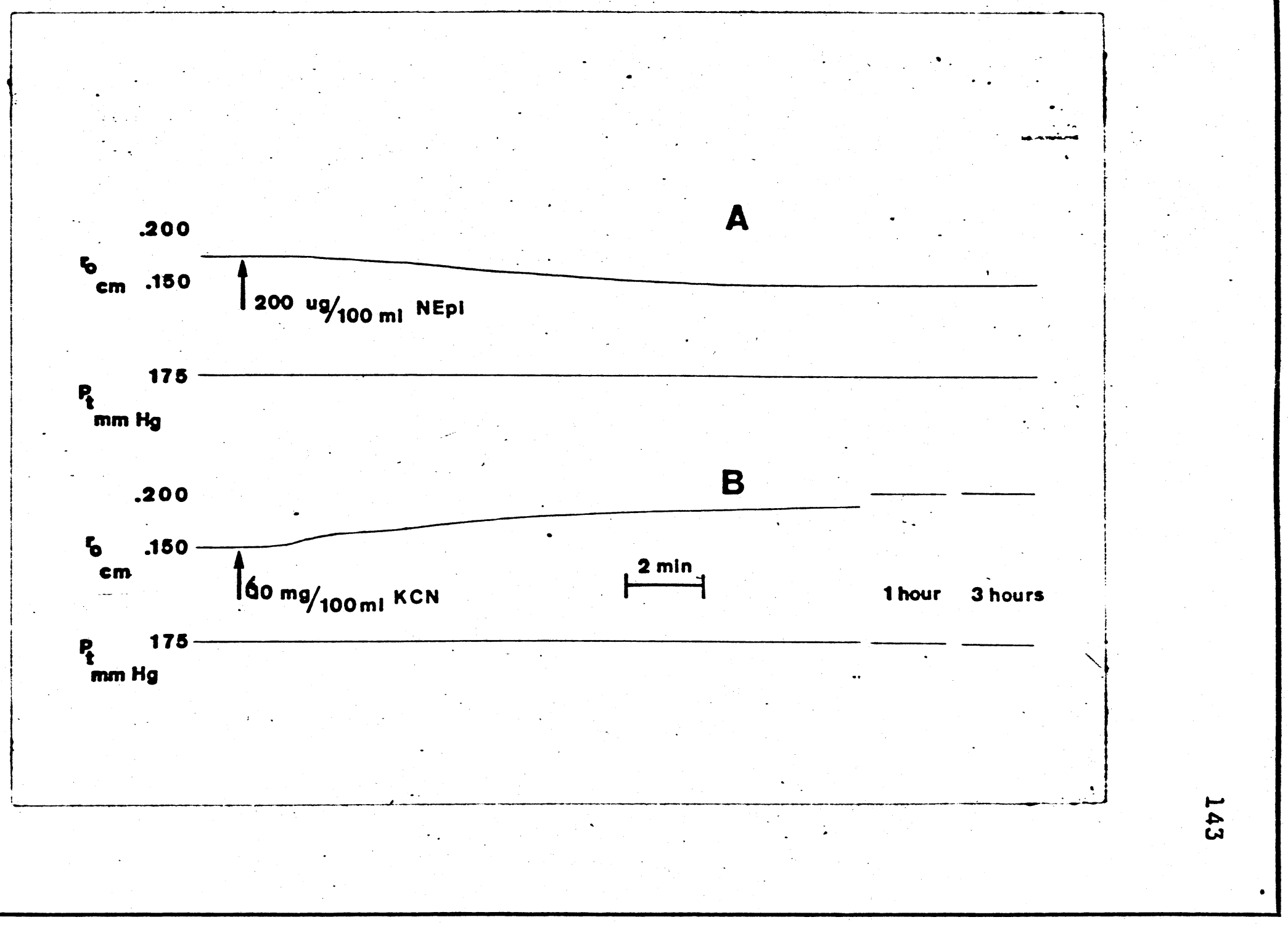




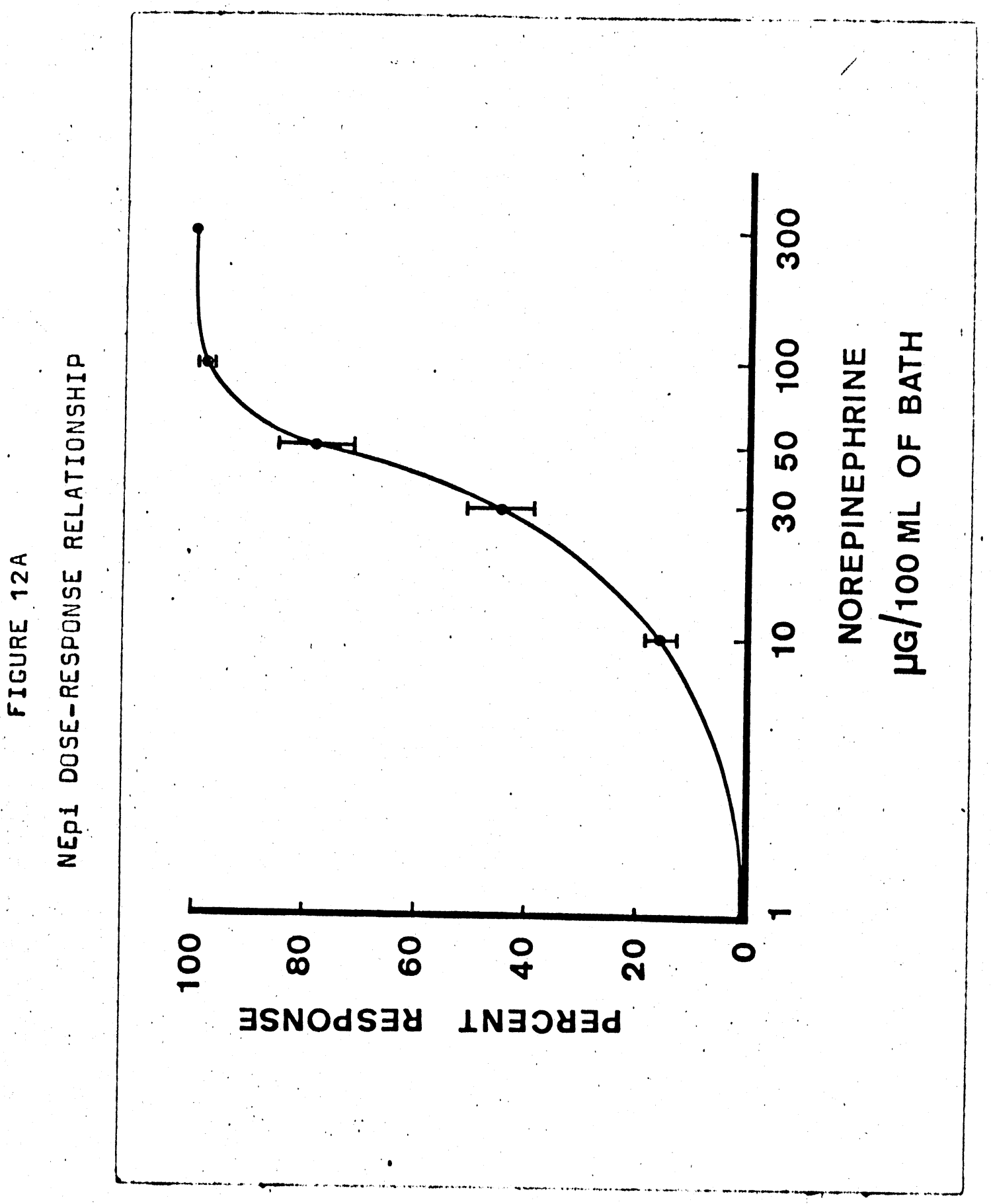


carotid arteries, and the brackets indicate standard errors. Maximum response was obtained with $300 \mu \mathrm{g} / 100 \mathrm{ml}$, of NEpi. In a few cases as much as $600 \mu \mathrm{g} / 100 \mathrm{ml}$ was given with no increase in response over that produced by $200 \mathrm{Jg} / 100 \mathrm{ml}$ dose. It was concluded, from these results, that a $200 \mu \mathrm{g} / 100 \mathrm{ml}$ dose of the drug could be used to strongly contract the muscle in subsequent experiments.

Figure 128 presents a KCN dose-response curve. The abscissa is the dose of KCN, plotted on a logarithmic scale, and the ordinate is the amplitude of dilation, expressed in percent. The 10 and $60 \mathrm{mg} / 100 \mathrm{ml}$ points on the graph represent the mean of 9 carotid arteries, and the $1.0 \mathrm{mg} / 100 \mathrm{ml}$ point represents 6 carotid arteries. Three additional arteries were treated with $0.1 \mathrm{mg} / 100 \mathrm{ml}$, but these were discontinued when it became evident that they produced negligible dilation. The brackets indicate standard errors. It was concluded from these results that $60 \mathrm{mg} / 100 \mathrm{ml} \mathrm{KCN}$ could be used to maximally inhibit smooth muscle contraction.

\section{Time-Course of KCN Action. Figure 13 presents} data describing the time-course of $K C N$ dilation for 5 carotid arteries. The abscissa is time in hours. It is broken to provide detail regarding the first 2 hours, and also to indicate the duration that the experiments were permitted to run. The ordinate is the amplitude of dilation expressed in percent, 


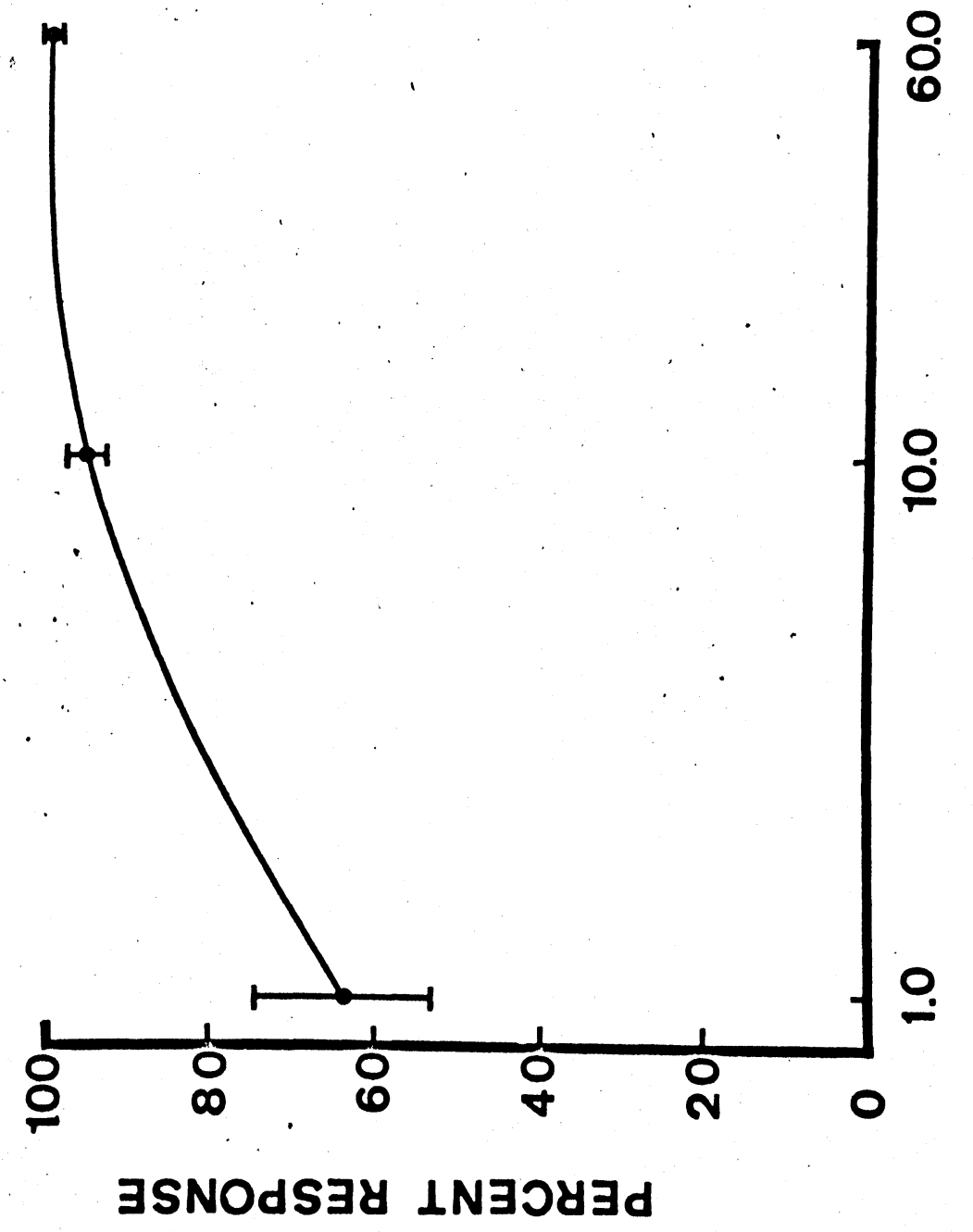


TIME COURSE OF KCN DILATION IN 5 ARTERIES

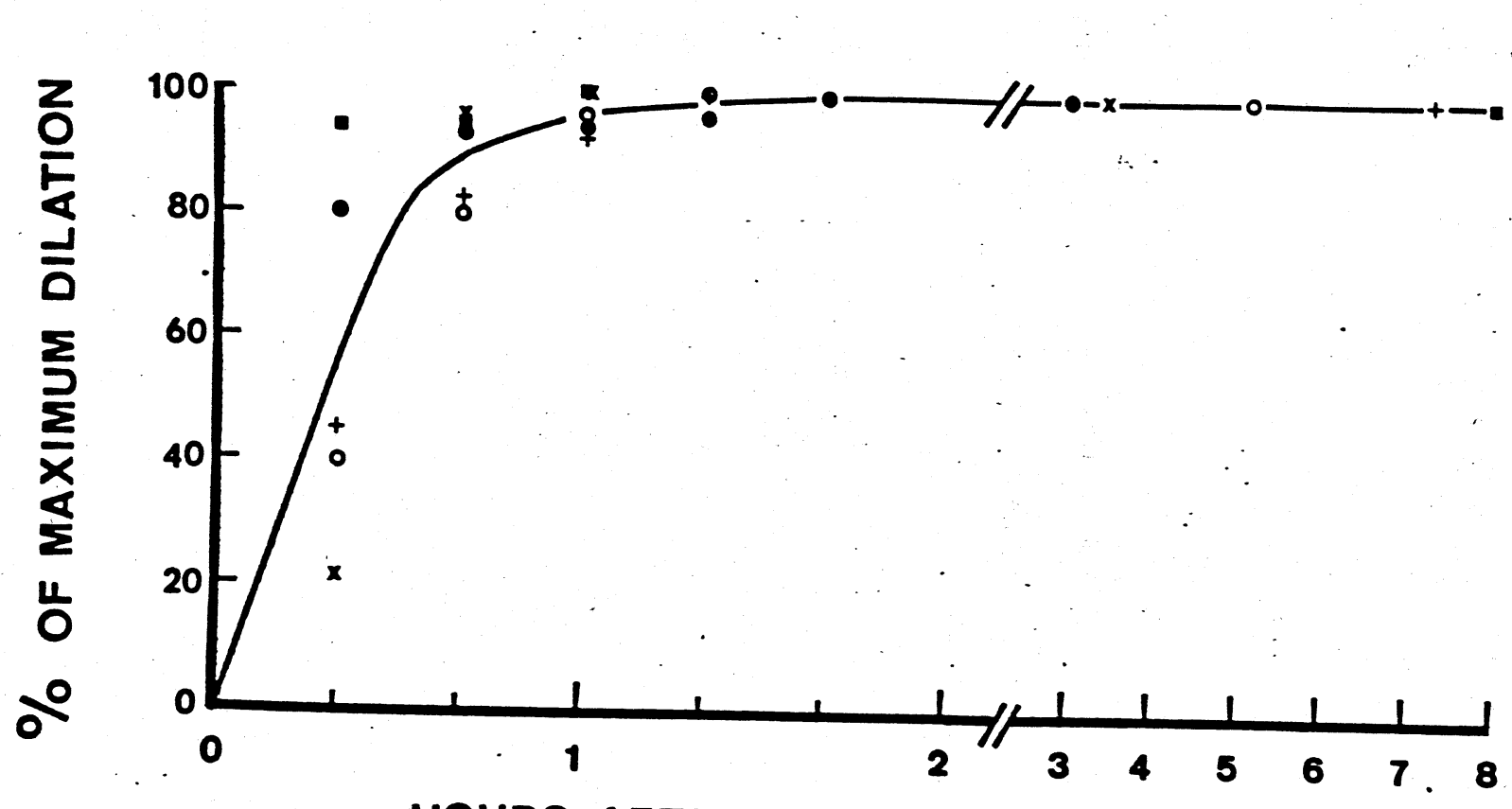

HOURS AFTER TREATMENT WITH KCN 
where maximum dilation was that observed at the end of each experiment. Each symbol represents a different aptery. Vessel dimensions were recorded continuously on the Polygraph, but only the responses observed at 20 minute intervals for the first 2 hours are plotted. Once a vessel reached its maximum radius it retained that dimension for the duration of the test. The final points shown on the graph incidate the duration of each individual experiment. Treatment with an additional dose of $200 \mathrm{mg} / 100 \mathrm{ml} \mathrm{KCN}$ failed to augment the dilation produced by exposure to $60 \mathrm{mg} / 100 \mathrm{ml} \mathrm{KCN}$ for many hours. It is clear from Figure 13 that approximately $95 \%$ of the maximum dilation occurs within the first hour after treatment. It was concluded, therefore, that waiting one hour after KCN treatment would be sufficient to consider vascular smooth muscle essentially inactive. Thus, 60 to 100 minutes were permitted to elapse before the final pressure-radius studies in each experiment were performed. In many experiments the vessels were treated with an additional $200 \mathrm{ug} / 100 \mathrm{ml}$ dose of NEpi after having undergone poisoning with cyanide for one hour or more. NEpi never elicited a contractile response after poisoning with $K C N$.

In several experiments, the pH of the bath was measured immediately before and after administration of NEpi and KCN. It was 7.4 before and after treatment with NEpi, but increased to 8.8 after treatment with cyanide. This high $\mathrm{pH}$ was 
probably important in relaxing or even killing the smooth muscle cells. Two arteries were treated, first with KCN buffered to $\mathrm{pH}$ 7.4, and then with Krebs-Ringer solution buffered to bring the bath to $\mathrm{pH}$ 8.8. Two additional arteries were treated, first with the alkaline Krebs-Ringer solution, and then with KCN buffered to $\mathrm{pH} 7.4$. In each case more than $90 \%$ of maximum dilation was produced by the first of two treatments. Data presented in the following section on the specificity of KCN action indicates that neither the $\mathrm{pH}$ nor the cyanide ion grossly disturbs the mechanical characteristics of the connective tissue elements.

3. Specificity of NEpi and KCN. The results of testing various arteries with $N E p i$ and $K C N$ are shown in Table I. These data show that vessels subjected to procedures intended to kill the smooth muscle never responded, in any way, to administration of NEpi. Vessels subjected to immersion in distilled water, exposure to an anoxic environment, to freezing or to heating failed, in all cases, to dilate in response to $K C N$. These vessels were tested in two ranges of pressure to determine which, if either, of the connective tissue elements might be effected by NEpi or KCN. Elastin bears most of the distending load in the $50-75 \mathrm{~mm} \mathrm{Hg}$ range, while collagen bears most of the load in the 175-200 $\mathrm{mm}$ Hg pressure range. The present data indicate that neither elastin, nor collagen, are obviously offected by NEpi or KCN because neither of these agents elicited 


\section{TABLE I}

\section{ACTION OF NEPI AND KCN ON ARTERIES}

SUBJECTED TO VARIOUS PRETREATMENT PROCEDURES

Procedure

\section{Perfused with}

$\mathrm{N}_{2}$ for 8 hours

Soaked in distilled $\mathrm{H}_{2} \mathrm{O}$ for

8 hours

Frozen for 8

hours

Heated to $50^{\circ}$

for 1 hour

Refrigerated

for 8 hours
Number of Arteries

$175-200$

$50-75$

$175-200$

50-75

175-200

125

225

$100-150$

\section{2}

Effect of NEpi

$$
\begin{aligned}
& \text { none } \\
& \text { none }
\end{aligned}
$$

$$
\begin{aligned}
& 3 \\
& 5
\end{aligned}
$$

3

3

1

3
3
5

none

none

none

none

none

none

dilation (3/4)

contraction
Effect of KCN

none

none

none

none

none

none

none

none

dilation dilation 
a response from arteries in which the contractile system had been inactivated by various methods.

4. Effects of NEpi and KCN on Wall Volume. Figure 14 presents a comparison of mean wall volumes in 10 arteries after successive treatment with NEpi and KCN. The abscissa is the arterial volume after treatment with NEpi, and the ordinate is the arterial volume after treatment with KCN. If there were no change in wall volume after these successive treatments, then each of the points would fall along the $45^{\circ}$ line of equality. Figure 14 indicates that there is a tendency for the wall volume to increase following poisoning with $\mathrm{KCN}$. A $16 \%$ increase was observed in a single case, although $6 \%$ was the average increase for the 10 vessels. The influence of NEpi and KCN on the wall volume was studied because the wall volume was used in all subsequent experiments to calculate the internal radil which correspond to the directly measured external radii. Equation 24 was used for this purpose; rearranged to solve for $r_{i}$,

$$
r_{i}=\sqrt{r_{0}^{2}-\frac{V}{\pi L}}
$$

Note that the square root of the wall volume is used. Therefore, the average increase in wall volume of $6 \%$, after treatment with cyanide, results in an average underestimation of about 
COMPARISON OF ARTERIAL WALL VOLUME AFTER NEPI, AND AFTER KCN

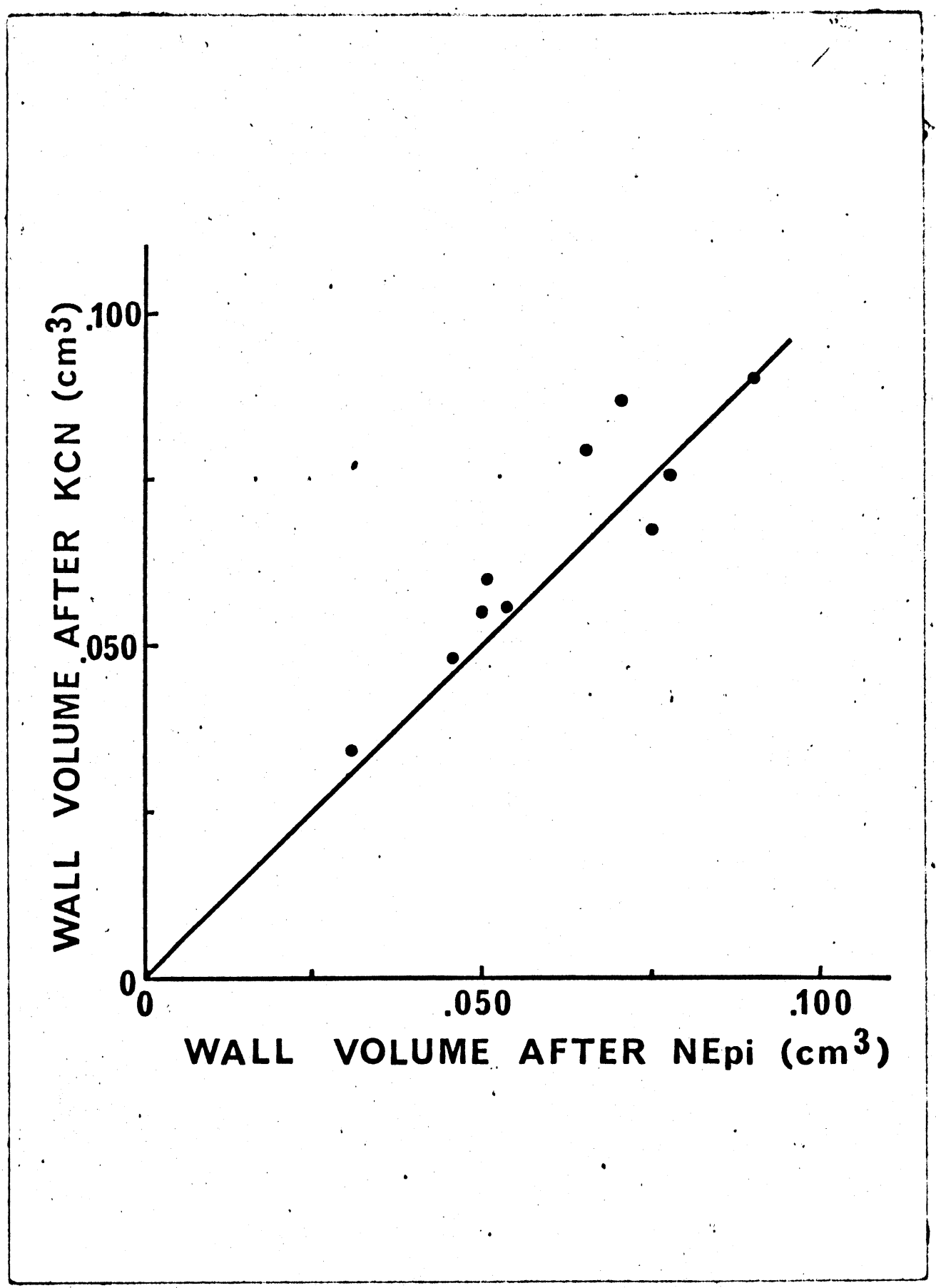


$2.5 \%$ in the calculation of internal radius after treatment with NEpi, because in subsequent experiments $x$-rays were taken only after poisoning with KCN. No attempt was made to correct for this error because of its small and inconsistent magnitude. Figure 15 presents the individual points comprising the mean wall volumes considered in Figure 14. Each symbol represents a different artery. The abscissa is external diameter, expressed as the percent of each individual vessel diameter at $50 \mathrm{~mm} \mathrm{Hg}$ following treatment with NEpi. It applies to both the left and right panels of the figure. The common ordinate is wall volume, expressed as the percent of mean wall volume for each individual vessel obtained following each treatment. Thus, the points cluster about their respective means, al though the mean following treatment with $K C N$ is actually $6 \%$ greater than the mean following treatment with NEp1. Each artery is represented by a different symbol. Five points were obtained for most arteries after NEpi, and after KCN, although in a fow cases only 4 points could be satisfactorily determined.

The dispersion of the individual points in those figures is obviously greater than the similar plot for untreated vessels illustrated in Figure 10. This is because the treated arteries underwent immersion in a Krebs-Ringer bath for 30 minutes with NEp1, and 60 minutes with KCN. These procedures washed away some of the lead oxide layer and rendered the $x$-rays 
FIGURE 15

ARTERIAL WALL VOLUME AFTER NEPI, AND AFTER KCN

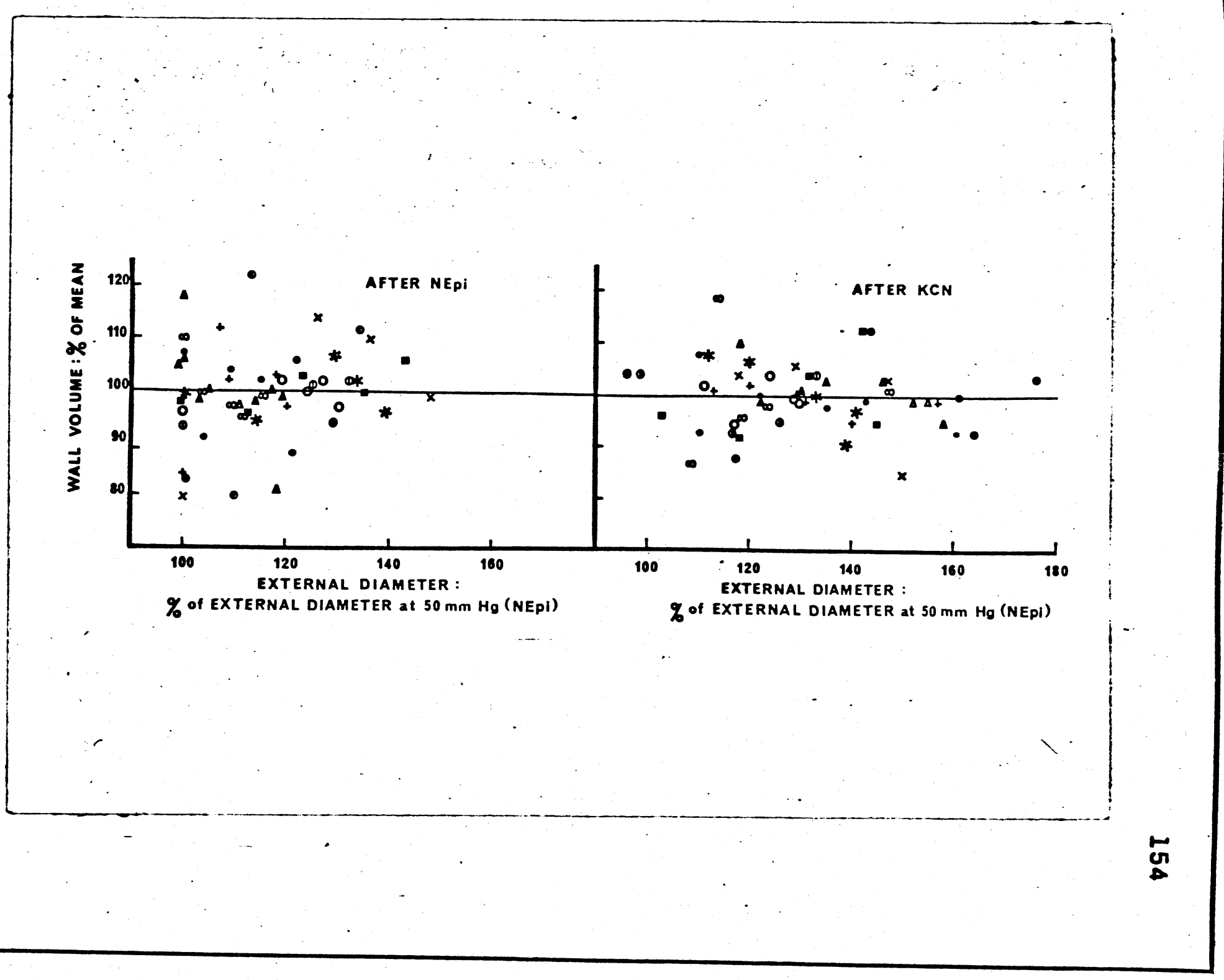


more difficult to measure. In spite of this, however, most of the scatter in Figure 15 is within $\pm 10 \%$ of the mean, /with just a fow points greater than that range. This figure indicates that there is little change in the slope of the wall volume curve after NEpi or KCN, suggesting that the presence of active smooth muscle does not alter the Poisson's ratio. There is a slight tendency for the slope of the wall volume curve after poisoning with KCN to fall from left to right, but this may have reflected a tendency for the wall to gain water after cyanide. C. Fixed-Length Arteries. 1. Shortening and Distention Behavior. Figure 16 presents a sample Polygraph record illustrating part of a pressure-radius cycle experiment.. Decreasing radius and increasing radius curves are shown in the pretreatment condition, after treatment with NEpi and after poisoning with cyanide. Figure $17 \mathrm{~A}$ and $18 \mathrm{~A}$ present pressureradius curves for 2 typical arteries subjected to a wide range of distending pressures. "Pre" indicates the behavior of the vessels during the pretreatment condition; "NEpi" after activation of the smooth muscle with NEpi; and "KCN" after poisoning with cyanide. It is evident that the vessels described in these figures were considerably relaxed before treatment with NEpi, because the Pre and KCN curves are similar. At low pressures and radil the vessels exhibited large changes in radius with each step change in pressure $(\Delta r / \Delta p)$. When the pressure and radius were increased, the $\left(\Delta_{r} / \Delta p\right)$ became progressively smaller. 
FIGURE 16

POLYGRAPH RECORDS IN PRETREATMENT STATE, AFTER TREATMENT WITH NEPI, AND AFTER POISONING WITH KCN.

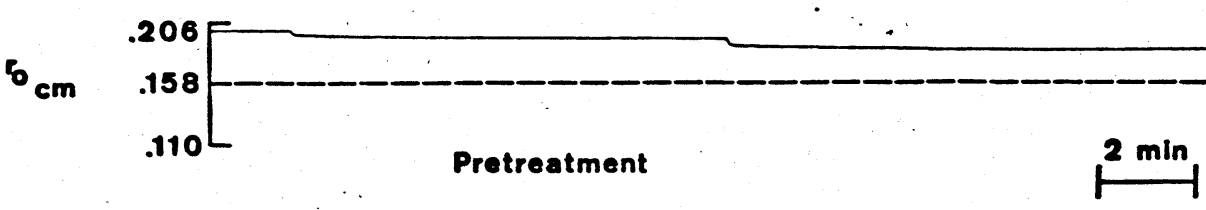

P $_{\mathrm{mm} \mathrm{Hg}}{ }_{0}^{125}$

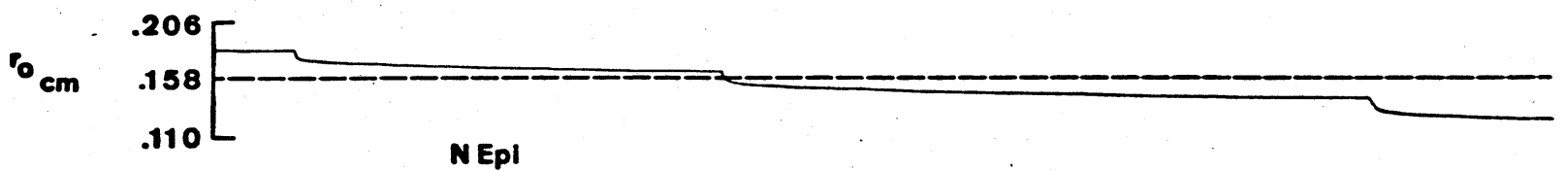

$P_{\mathrm{mm} \mathrm{Hg}}{ }_{0}^{125}[$

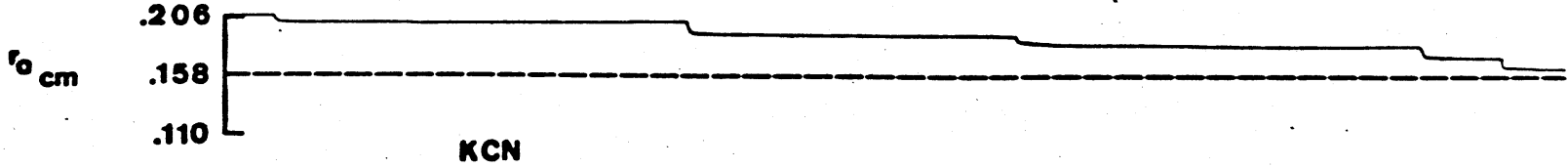

$P_{\mathrm{mm} \mathrm{Hg}}{ }_{0}^{125}[$ 
FIGURE $17 \mathrm{~A}$

ARTERY 1: PRESSURE-RADIUS CURVES IN THE PRETREATIIENT CONDITION AND AFTER TREATMENT WITH NEPI AND KCN.

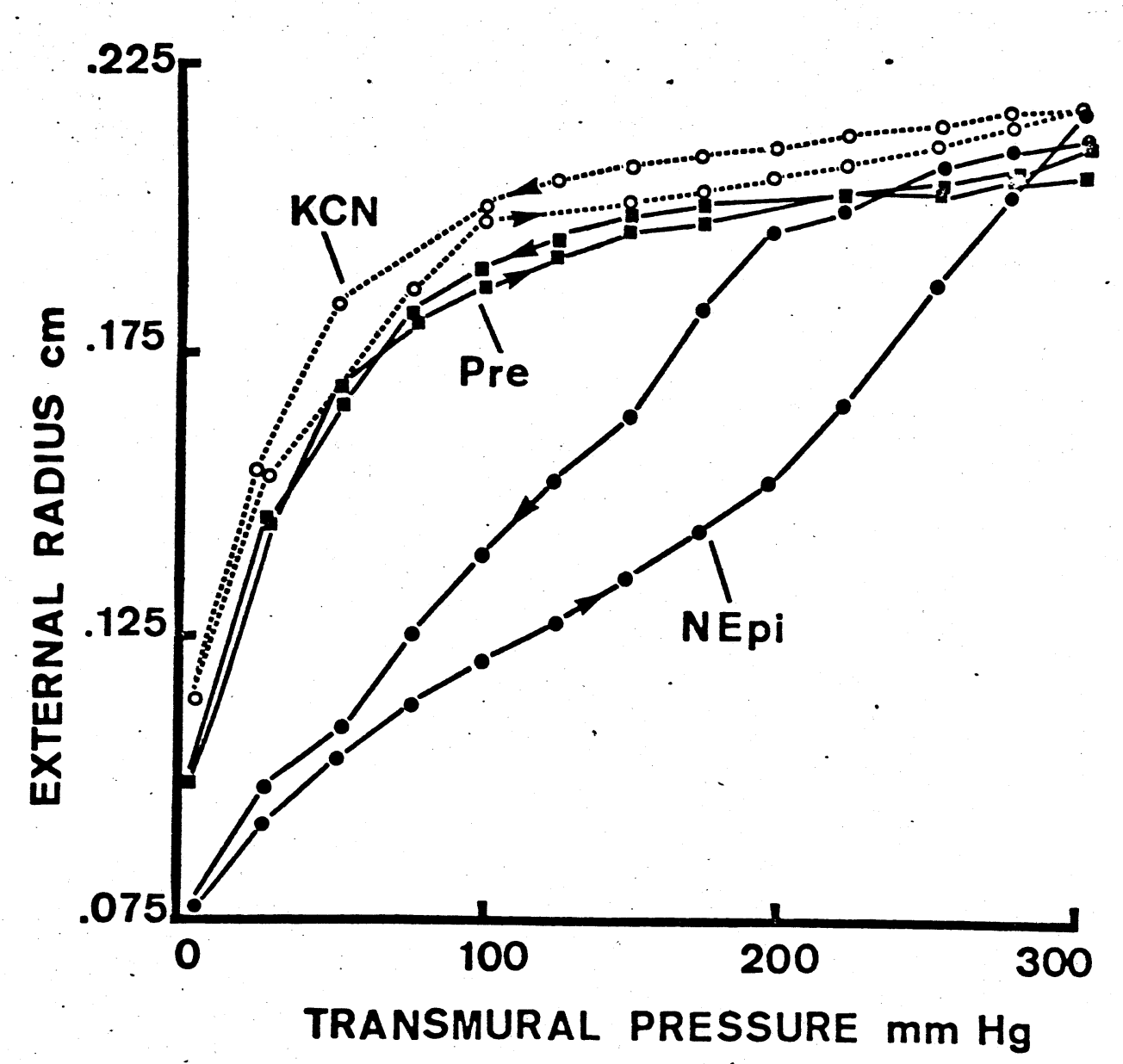


ARTERY 2: PRESSURE-RADIUS CURVES IN THE PRETREATMENT CONDITION AND AFTER TREATMENT WITH NEPI AND KCN.

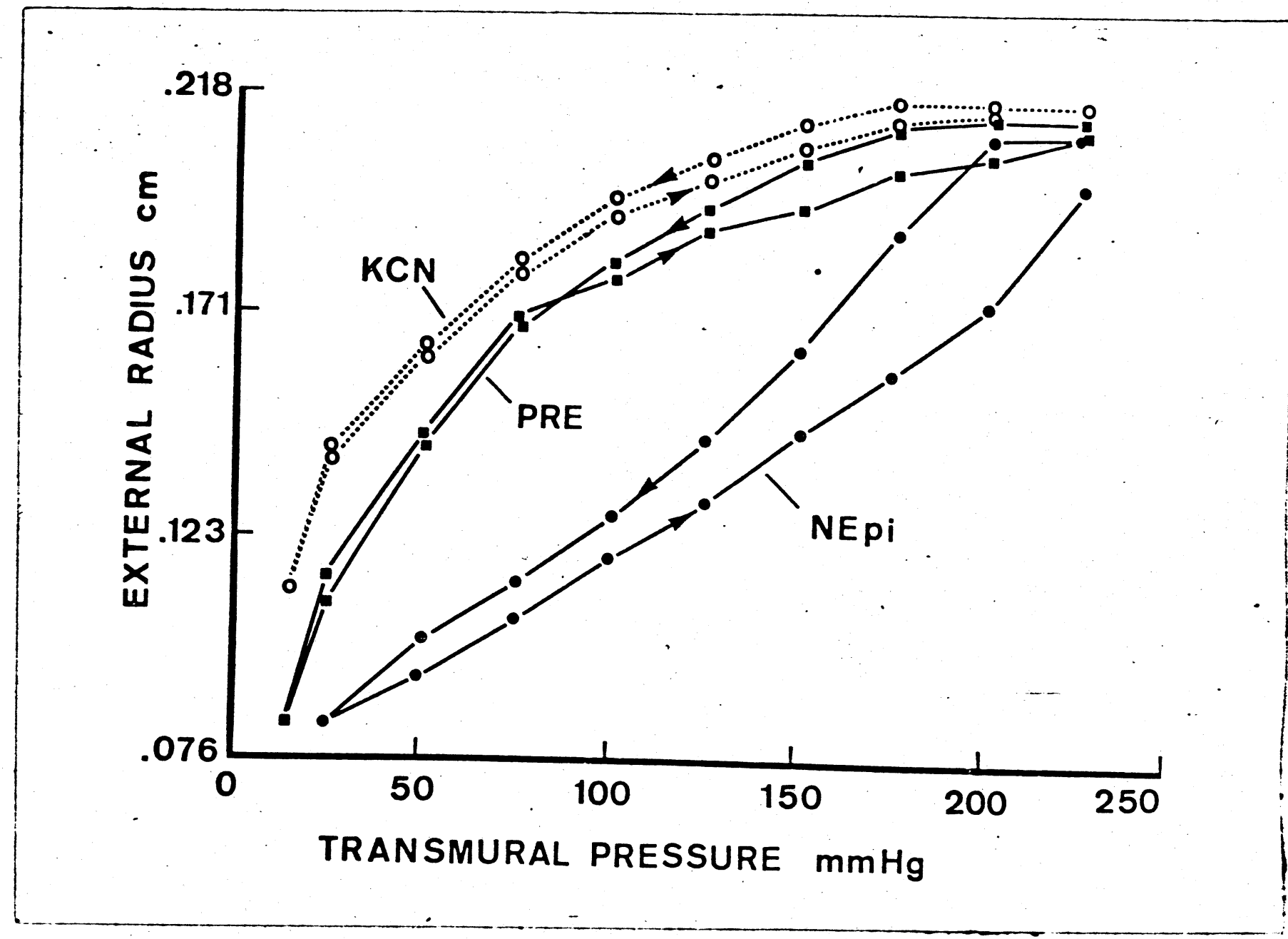


The dependence of $(\Delta r / \Delta P)$ upon pressure or radius was most apparent in the pretreatment condition and after poisoning with cyanide. Treatment with NEpi caused a gradual contraction so that the $(\Delta r / \Delta P)$ was less dependent upon radius or pressure. Treatment with NEp1 also greatly increased the amplitude, or width, of the extension-retraction hysteresis $100 p$.

The amplitude of muscle shortening is the difference in radius at any pressure between the vessel following treatment with NEpi and following poisoning with cyanide. It is clear from figures $17 \mathrm{~A}$ and $18 \mathrm{~A}$ that the amplitude of muscle shortening is great at moderate pressures, and exhibits a maximum at or below physiological pressures.

Table I presents similar data for a total of 16 arteries. Maximum active stress, the strain at which the maximum stresses occurred, and the pressure at which the maximum amplitude of shortening occurred are presented. Means and standard errors are included at the bottom of the Table. The mean peak active stress was $.88 \pm .11 \times 10^{6} \mathrm{dynes} / \mathrm{cm}^{2}$. It occurred at a mean circumferential strain of $.57 \pm .04$. Maximum amplitude of muscle shortening occurred at a mean pressure of $62 \pm 4.8 \mathrm{~mm} \mathrm{Hg} \cdot$ D. Extension Behavior. It is evident from the pressure-radius curves presented in Figures $17 \mathrm{~A}$ and $18 \mathrm{~A}$ that each radius is associated with a higher pressure during the distertion sequences than during the shortening sequences. This is 
ARTERY 18 SHORTENING STRESS-STRAIN CURVES AFTER NEPI, KCN,

AND THE DIFFERENCE BETWEEN THEM (ACTIVE MUSCLE)

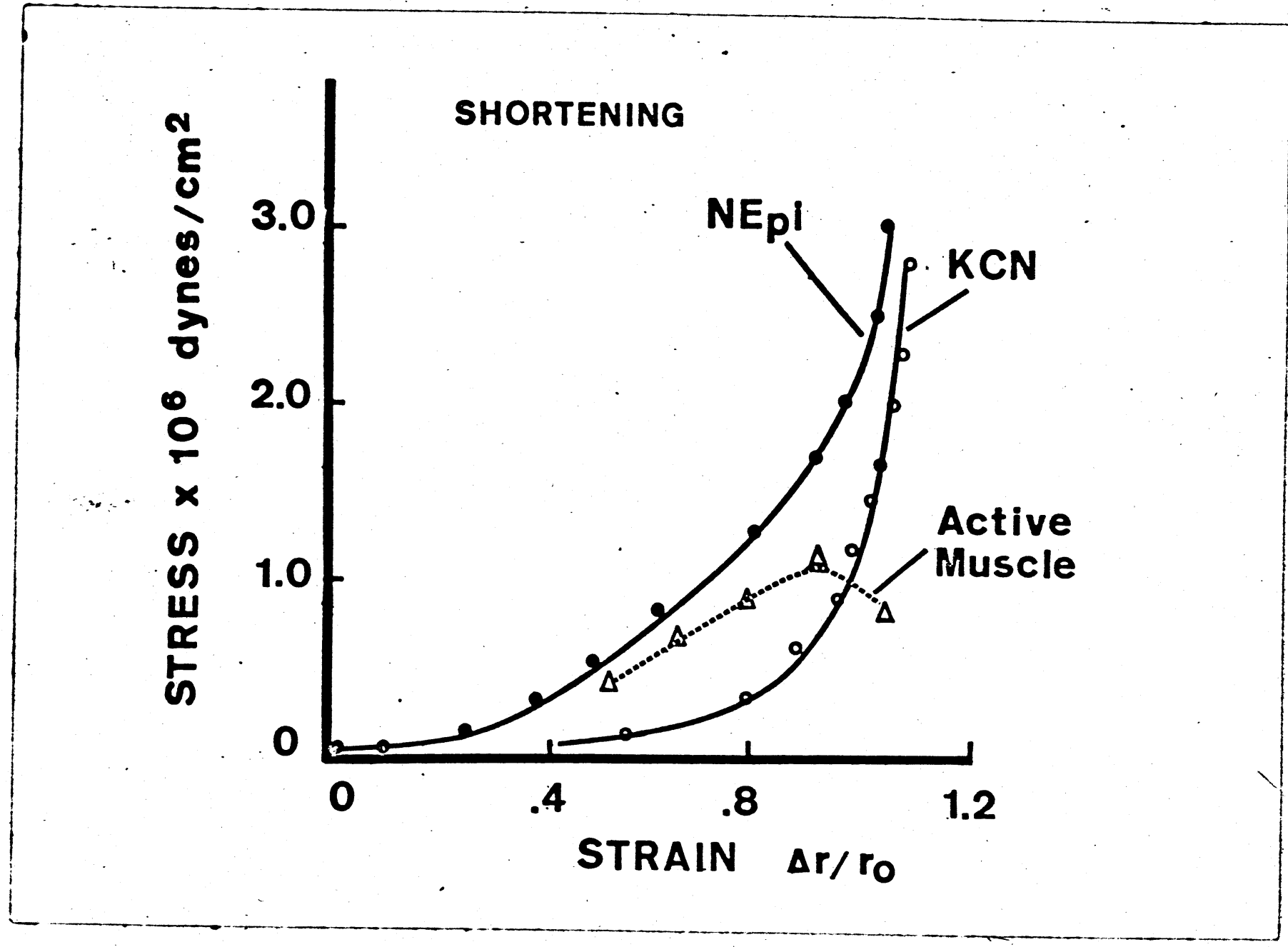


FIGURE 188

ARTERY 2: SHORTENING STRESS-STRAIN CURVES AFTER NEP1, KCN AND THE DIFFERENCE BETWEEN THEM (ACTIVE MUSCLE).

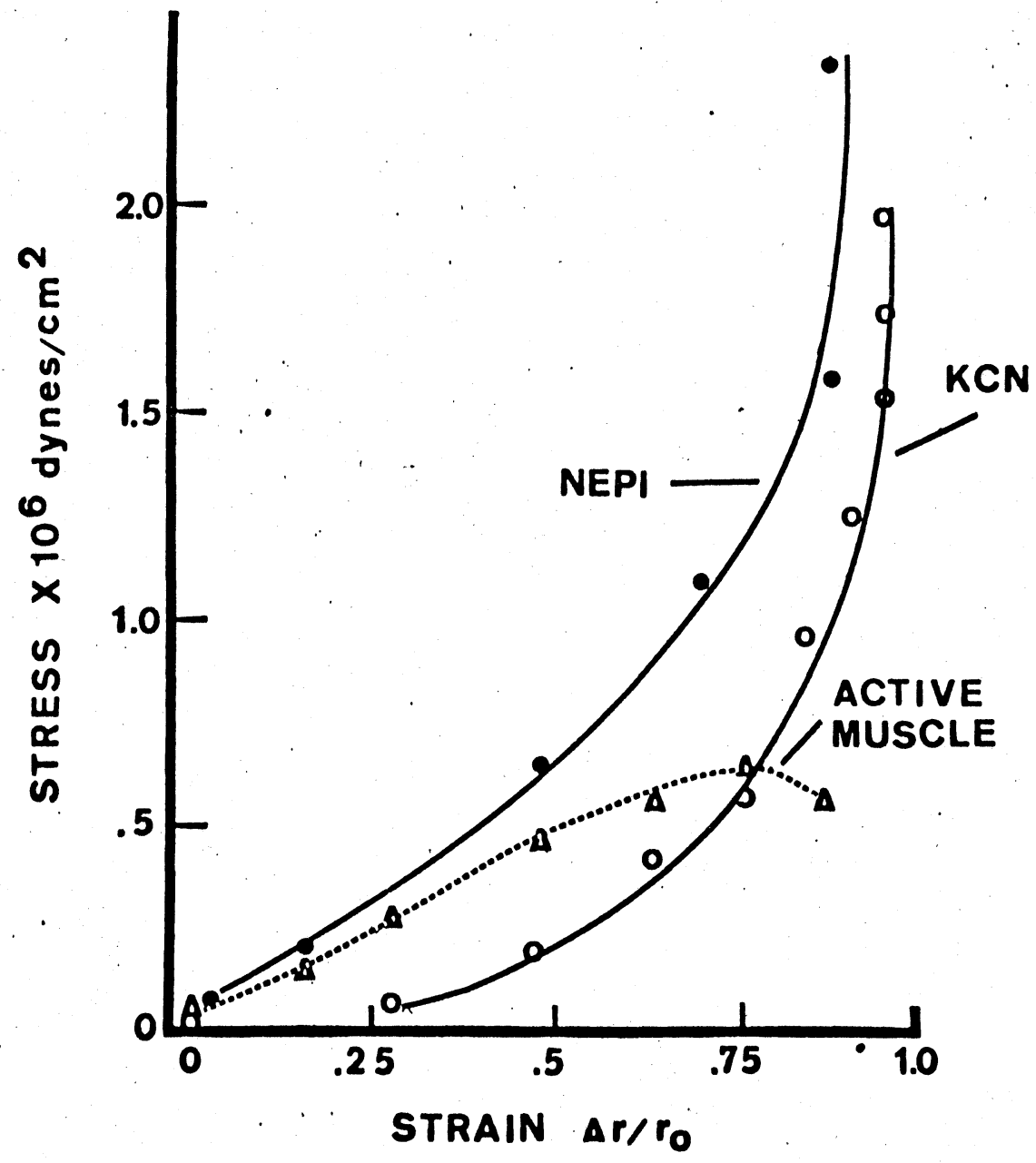




\section{TABLE II}

SHORTENING CHARACTERISTICS OF NOREPINEPHRINE-ACTIVATED CAROTID ARTERIES

\begin{tabular}{|c|c|c|c|}
\hline Artery & $\begin{array}{l}\text { Maximum Active Stress } \\
\qquad\left(\times 10^{6} \text { dynes } / \mathrm{cm}^{2}\right)\end{array}$ & $\begin{array}{l}\text { Strain at Maximum } \\
\text { Active Stress } \\
\left(\Delta r / r_{0}\right)\end{array}$ & $\begin{array}{l}\text { Pressure At } \\
\text { Maximum Shortening } \\
(\mathrm{mm} \mathrm{Hg})\end{array}$ \\
\hline $\begin{array}{r}1 \\
2 \\
3 \\
4 \\
5 \\
6 \\
7 \\
8 \\
9 \\
10 \\
11 \\
12 \\
13 \\
14 \\
15 \\
16\end{array}$ & $\begin{array}{r}1.20 \\
.60 \\
.40 \\
.70 \\
.60 \\
1.90 \\
1.70 \\
.50 \\
.50 \\
.30 \\
1.20 \\
.90 \\
.75 \\
.91 \\
1.10 \\
.86\end{array}$ & $\begin{array}{l}.80 \\
.90 \\
.55 \\
.53 \\
.50 \\
.30 \\
.73 \\
.68 \\
.47 \\
.34 \\
.74 \\
.65 \\
.36 \\
.40 \\
.74 \\
.50\end{array}$ & $\begin{array}{l}50 \\
75 \\
75 \\
75 \\
90 \\
75 \\
50 \\
50 \\
75 \\
25 \\
75 \\
75 \\
50 \\
25 \\
55 \\
75\end{array}$ \\
\hline $\begin{array}{l}\text { Mean } \\
\text { Standard Error }\end{array}$ & $\begin{array}{r}.88 \\
\pm .11\end{array}$ & $\begin{array}{r}.57 \\
\pm .04\end{array}$ & $\begin{array}{c}62 \\
\pm 4.8\end{array}$ \\
\hline
\end{tabular}


especially pronounced after treatment with NEpi. Because the circumferential stress is directly related to the pressure at any given radius (equation 4b), it is apparent that the stress is greater at any strain during the distention sequence than during the shortening sequence.

Figures $17 \mathrm{C}$ and $18 \mathrm{C}$ present the distention data in the form of stress-strain curves. The slopes of these curves were determined graphically. Seventy-five percent of the value of the slope was used as a measure of the elastic modulus, in accord with equation 23, 1.8. $E_{C}=\frac{\Delta \sigma_{C}}{\Delta \varepsilon_{C}}\left(1-v^{2}\right)$. Since Poisson's ratio is equal to 0.5 , the expression $\left(1-v^{2}\right)$ is equal to 0.75 . These elastic moduli are plotted as a function of strain in Figures 170 and 180 . It may be observed that the elastic modulus of the arteries is higher after treatment with NEpi, than after poisoning with cyanide. This is found at all but the largest strains. The consistency of this finding is demonstrated by the data presented in Table IIIA. Figures $17 E$ and $18 E$ present the same elasticity data plotted as a function of transmural pressure. Contrary to the data presented in the stress-elasticity graphs, these figures indicate that the arteries are less elastic at any given pressure after treatment with NEpl than after poisoning with cyanide.

Interpretation of the pressure-elasticity data presented in figures $17 E$ and $I 8 E$ is complicated by the fact that 
ARTERY 1: DISTENTION STRESS-STRAIN CURVES

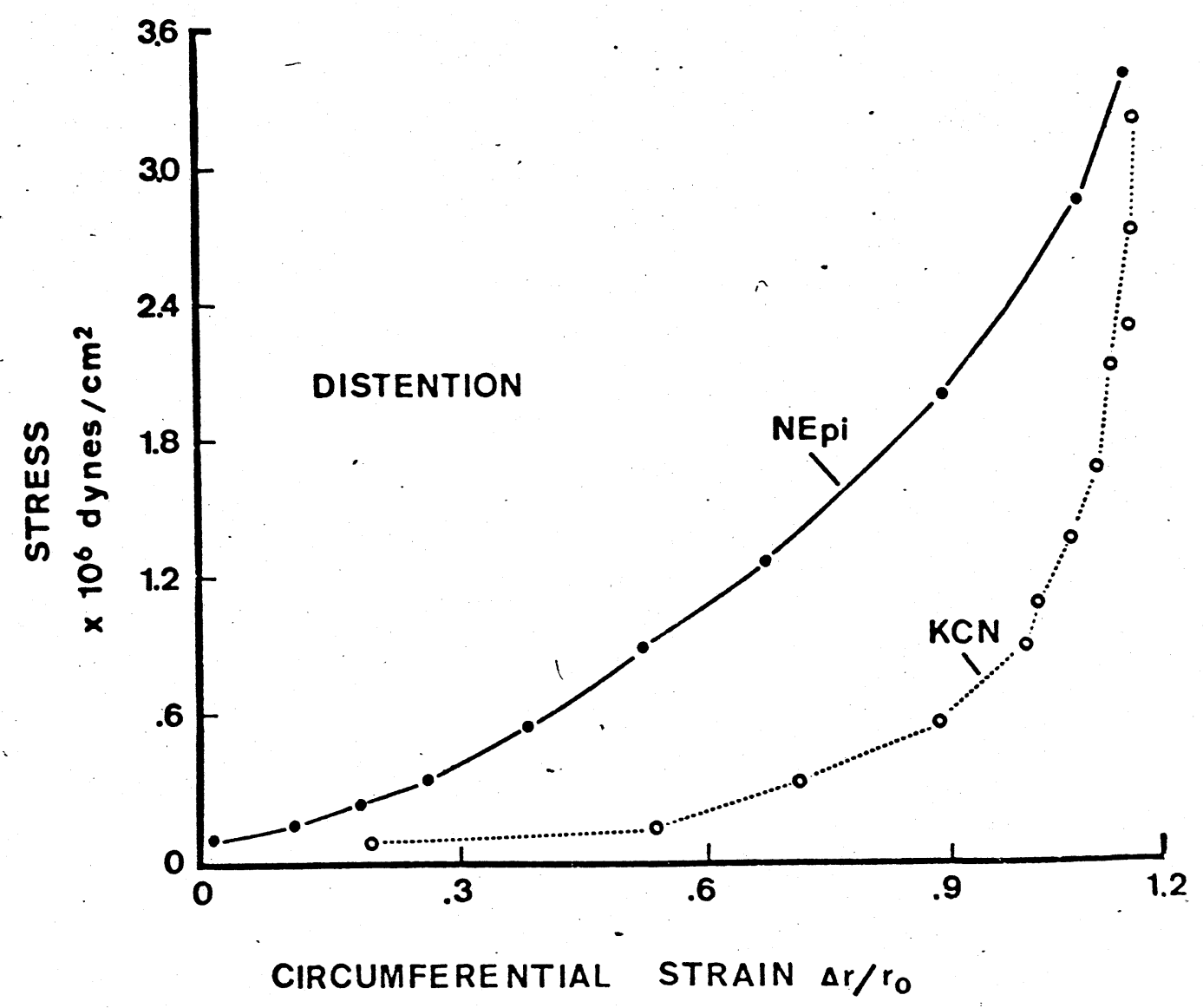


FIGURE $18 C$

ARTERY 2: DISTENTION STRESS-STRAIN CURVES

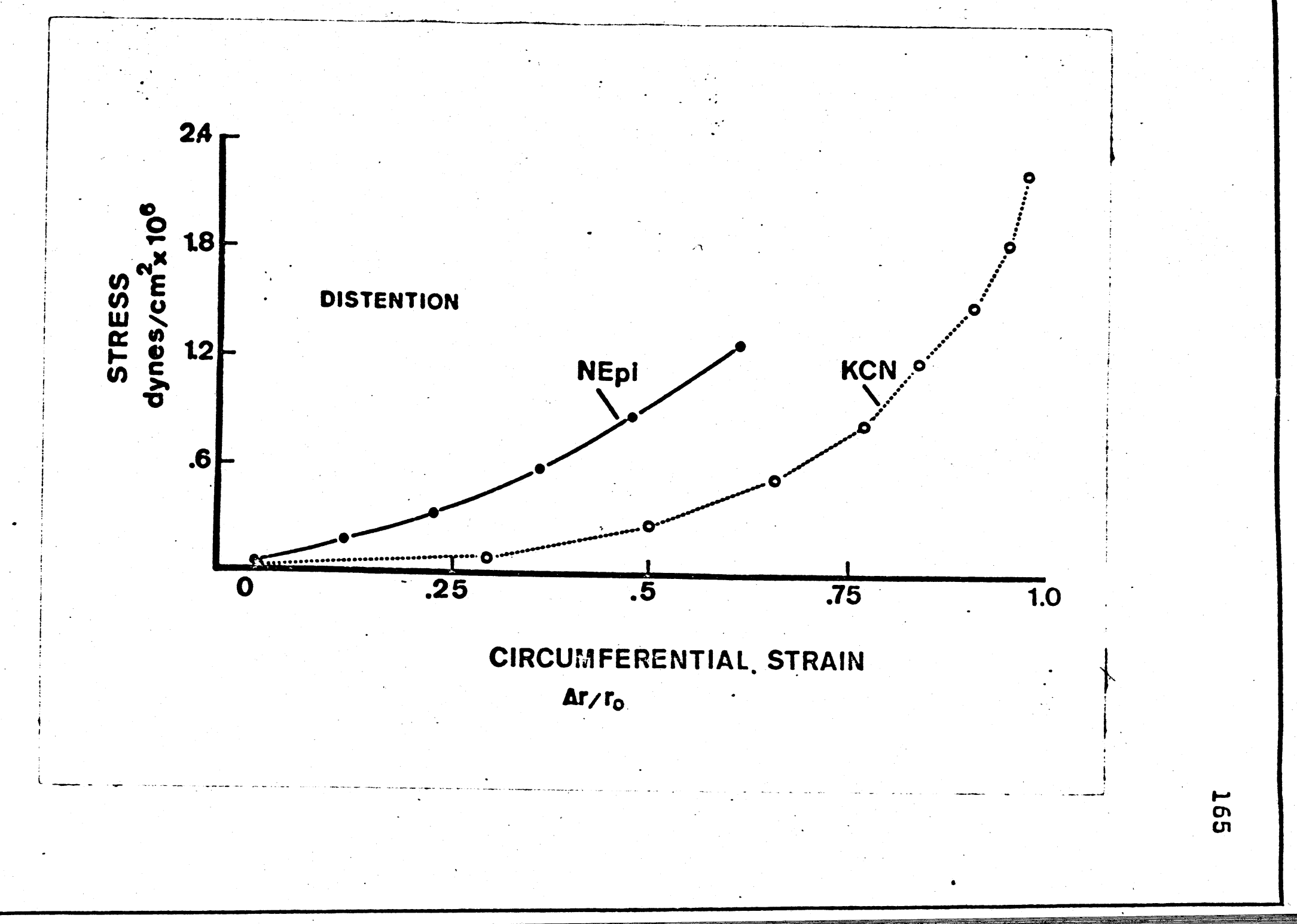


ARTERY 1: STRAIN-ELASTIC MODULUS CURVES FOR DISTENTION BEHAVIOR. THE ARROUS. INDICATE THE STRAINS AT $100 \mathrm{~mm} \mathrm{Hg}$.

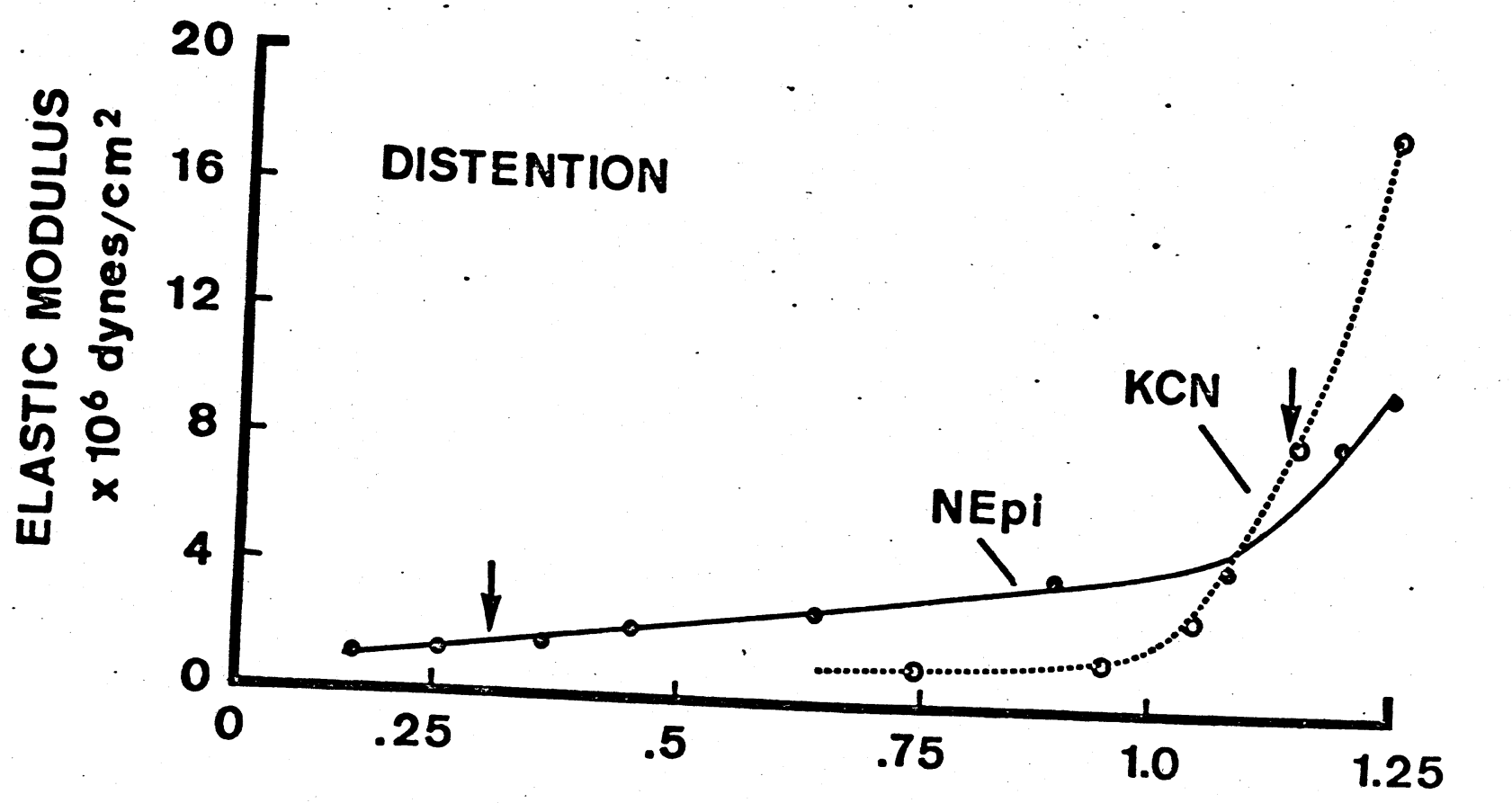

CIRCUMFERENTIAL STRAIN Ar/ro 
ARTERY 2: STRAIN-ELASTIC MODULUS CURVES FOR DISTENTION BEHAVIOR

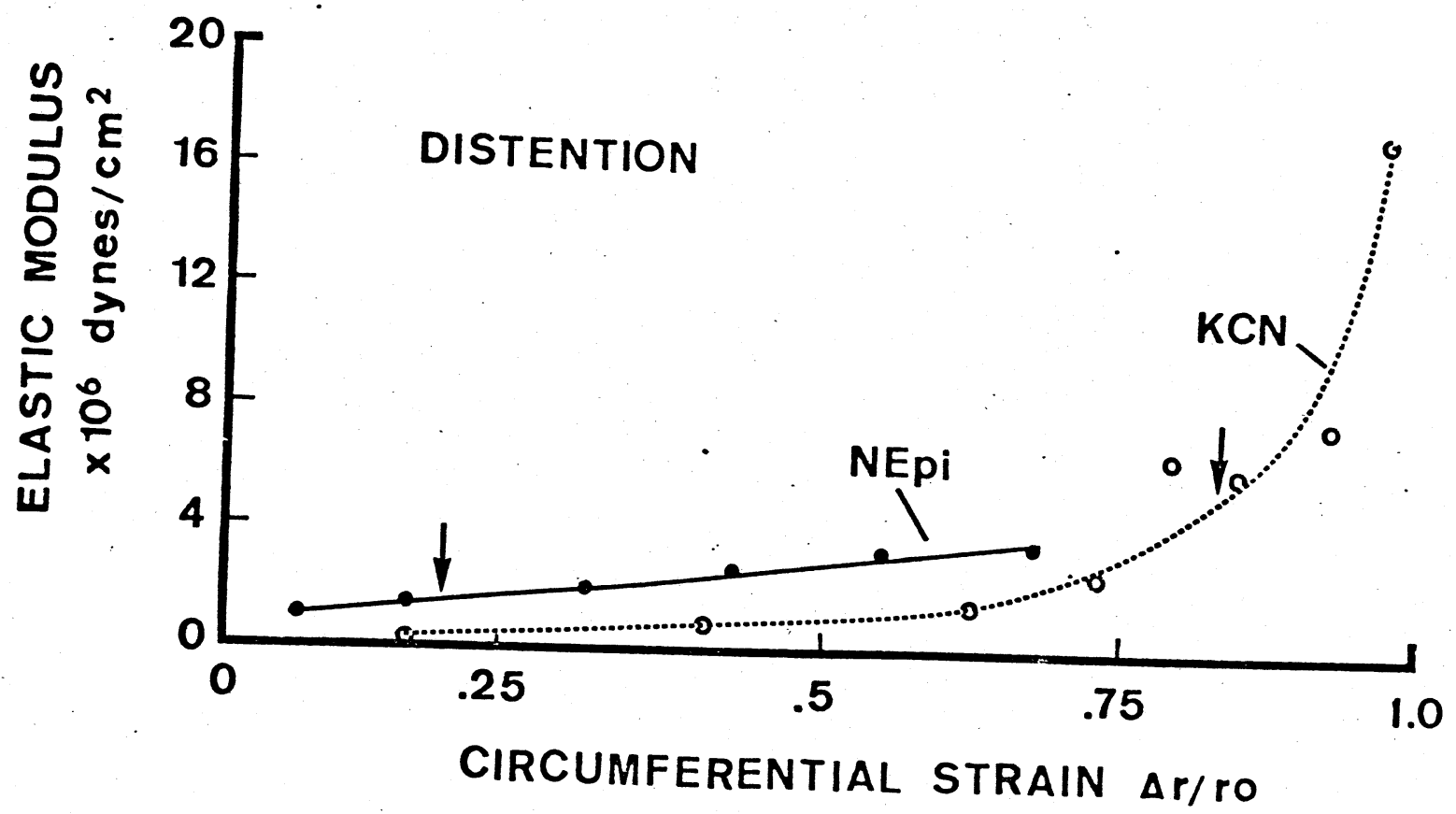


TABLE III $A$

ELASTIC MODULUS ( $x 10^{6}$ dynes $/ \mathrm{cm}^{2}$ )

AS A FUNCTION OF STRAIN

Strain

\begin{tabular}{|c|c|c|c|c|c|c|c|c|c|c|c|c|c|}
\hline Artery & & $: 1$ & .2 & .3 & .4 & .5 & .6 & .7 & .8 & .9 & 1.0 & $1: 1$ & 1.2 \\
\hline 1 & $\begin{array}{l}\text { NEpi } \\
\text { KCN }\end{array}$ & 1.3 & $\begin{array}{c}1.7 \\
-\end{array}$ & $\begin{array}{c}2.0 \\
-\end{array}$ & $\begin{array}{c}2.2 \\
-\end{array}$ & $\begin{array}{c}2.5 \\
-\end{array}$ & $\begin{array}{r}2.5 \\
1.2\end{array}$ & $\begin{array}{l}3.8 \\
1.2\end{array}$ & $\begin{array}{l}3.8 \\
1.5\end{array}$ & $\begin{array}{l}3.8 \\
3.0\end{array}$ & $\begin{array}{l}4.7 \\
3.0\end{array}$ & $\begin{array}{l}8.5 \\
8.5\end{array}$ & 19.0 \\
\hline 2 & $\begin{array}{l}\text { NEpi } \\
\text { KCN }\end{array}$ & $\begin{array}{c}1.0 \\
-\end{array}$ & $\begin{array}{r}1.2 \\
.3\end{array}$ & $\begin{array}{r}1.8 \\
.6\end{array}$ & $\begin{array}{l}2.4 \\
.87\end{array}$ & $\begin{array}{l}3.0 \\
1.3\end{array}$ & - & 2.7 & - & 7.9 & $\overline{17.4}$ & & \\
\hline 3 & $\begin{array}{l}\text { NEpi } \\
\text { KCN }\end{array}$ & $\begin{array}{r}6.8 \\
.6\end{array}$ & $\begin{array}{l}5.2 \\
1.7\end{array}$ & $\begin{array}{l}7.0 \\
2.8\end{array}$ & $\begin{array}{r}11.2 \\
3.6\end{array}$ & $\begin{array}{l}1.61 \\
7.0\end{array}$ & $\begin{array}{r}21 . \\
8.3\end{array}$ & $\begin{array}{r}26.9 \\
13.4\end{array}$ & $\begin{array}{l}35 \\
21.0\end{array}$ & & & & \\
\hline 4 & $\begin{array}{l}\text { NEpi } \\
\text { KCN }\end{array}$ & $\begin{array}{r}2.6 \\
.6\end{array}$ & $\begin{array}{l}3.0 \\
1.4\end{array}$ & $\begin{array}{l}3.0 \\
2.1\end{array}$ & $\begin{array}{l}3.0 \\
3.0\end{array}$ & $\begin{array}{l}5.0 \\
4.0\end{array}$ & $\begin{array}{l}7.0 \\
5.5\end{array}$ & & & & & & \\
\hline 6 & $\begin{array}{l}\text { NEpi } \\
\text { KCN }\end{array}$ & $\begin{array}{l}2.5 \\
1.9\end{array}$ & $\begin{array}{l}6.0 \\
2.5 .\end{array}$ & $\begin{array}{l}8.0 \\
6.0\end{array}$ & $\begin{array}{l}17 \\
8.0\end{array}$ & $\begin{array}{l}17 \\
19\end{array}$ & & & & & & & \\
\hline 7 & $\begin{array}{l}\text { NEpi } \\
\text { KCN }\end{array}$ & - & $\begin{array}{l}3.2 \\
1.4\end{array}$ & $\begin{array}{l}4.8 \\
2.3\end{array}$ & $\begin{array}{l}5.7 \\
2.3\end{array}$ & $\begin{array}{c}10.2 \\
4.75\end{array}$ & $\begin{array}{r}10.5 \\
8.6\end{array}$ & 14.0 & & & & & \\
\hline 10 & $\begin{array}{l}\text { NEpi } \\
\text { KCN }\end{array}$ & $\begin{array}{c}2.2 \\
-\end{array}$ & $\begin{array}{l}3.5 \\
1.0\end{array}$ & $\begin{array}{l}5.0 \\
2.2\end{array}$ & $\begin{array}{l}7.0 \\
4.0\end{array}$ & $\begin{array}{l}7.5 \\
9.0\end{array}$ & & & & & & & \\
\hline 11 & $\begin{array}{l}\text { NEpi } \\
\text { KCN }\end{array}$ & .2 & .85 & .85 & $\begin{array}{r}1.2 \\
.6\end{array}$ & $\begin{array}{r}2.1 \\
.6\end{array}$ & $\begin{array}{r}2.1 \\
.6\end{array}$ & $\begin{array}{l}3.5 \\
1.37\end{array}$ & $\begin{array}{l}2.6 \\
1.37\end{array}$ & $\begin{array}{l}2.6 \\
2.7\end{array}$ & 5.5 & 9.0 & \\
\hline
\end{tabular}

(Continued) 
(Continued Table III A)

Strain

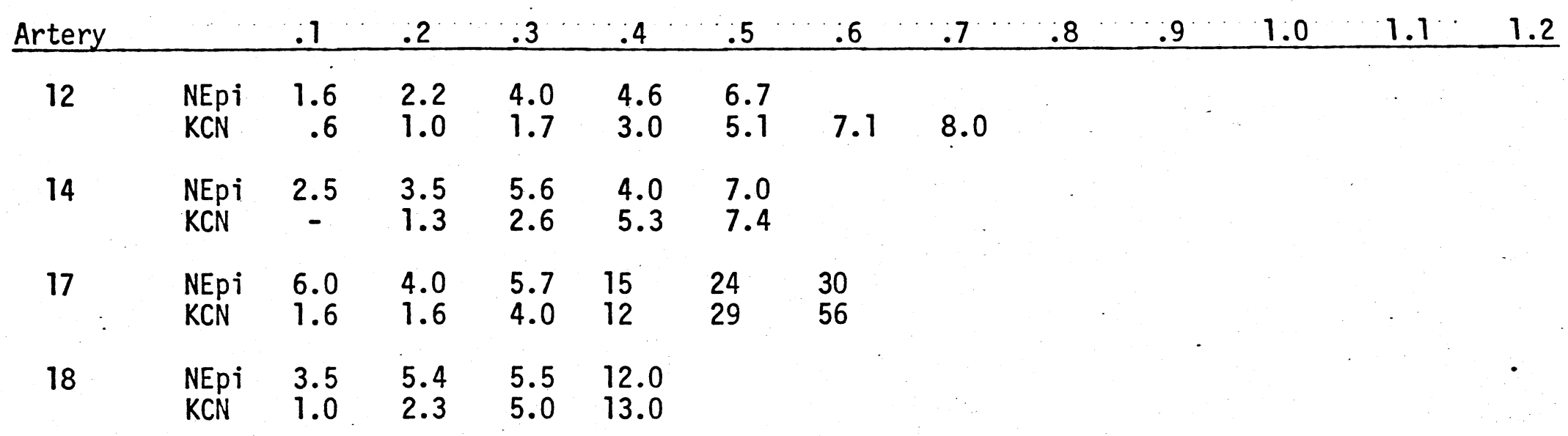

TABLE III $B$

ELASTIC MODULUS ( $x 10^{6}$ dynes $/ \mathrm{cm}^{2}$ )

AS A FUNCTION OF PRESSURE

Pressure

\begin{tabular}{ccccccccccr} 
Artery & & 25 & 50 & 75 & 100 & 125 & 150 & 175 & 200 \\
\hline 1 & NEpi & - & .8 & 1.3 & 1.5 & 1.8 & 2.0 & 2.3 & 2.5 \\
& KCN & .3 & 1.2 & 1.5 & 3.0 & 3.5 & 8.4 & 8.5 & 19.5 \\
2 & NEpi & - & - & 1.0 & 1.1 & 1.6 & 2.1 & 2.7 & 3.0 \\
& KCN & .6 & 1.2 & 2.2 & 4.5 & 6.3 & 6.2 & 12.5 & 17.4 \\
3 & NEpi & 1.2 & 6.8 & 5.2 & 7.0 & 11.2 & 16.1 & 23.3 & 26.9 \\
& KCN & 1.7 & 3.6 & 8.3 & 13.4 & 20.0 & 22.3 & - & -
\end{tabular}

(Continued) 
(Continued Table III B)

Pressure

\begin{tabular}{|c|c|c|c|c|c|c|c|c|c|}
\hline Artery & & 25 & 50 & 75 & 100 & 125 & 150 & 175 & 200 \\
\hline 4 & $\begin{array}{l}\text { NEpi } \\
\text { KCN }\end{array}$ & $\begin{array}{l}1.9 \\
1.0\end{array}$ & $\begin{array}{l}1.9 \\
1.8\end{array}$ & $\begin{array}{l}2.3 \\
2.6\end{array}$ & $\begin{array}{l}2.6 \\
3.5\end{array}$ & $\begin{array}{l}3.0 \\
4.7\end{array}$ & $\begin{array}{l}3.1 \\
5.5\end{array}$ & $\begin{array}{c}4.0 \\
-\end{array}$ & $\begin{array}{c}6.0 \\
-\end{array}$ \\
\hline 6 & $\begin{array}{l}\text { NEpi } \\
\text { KCN }\end{array}$ & $2.2^{-}$ & $\begin{array}{l}2.5 \\
4.3\end{array}$ & $\begin{array}{l}5.2 \\
7.0\end{array}$ & $\begin{array}{r}8.0 \\
13.0\end{array}$ & $\begin{array}{r}8.0 \\
19.0\end{array}$ & $\begin{array}{c}12.0 \\
-\end{array}$ & $\begin{array}{c}17.0 \\
-\end{array}$ & $\begin{array}{c}17.0 \\
-\end{array}$ \\
\hline 7 & $\begin{array}{l}\text { NEpi } \\
\text { KCN }\end{array}$ & 1.9 & $\begin{array}{l}3.2 \\
3.5\end{array}$ & $\begin{array}{l}4.0 \\
6.6\end{array}$ & $\begin{array}{r}5.2 \\
11.2\end{array}$ & $\begin{array}{r}5.7 \\
14.0\end{array}$ & $\begin{array}{c}10.2 \\
-\end{array}$ & $\begin{array}{c}10.2 \\
-\end{array}$ & $\begin{array}{c}10.2 \\
-\end{array}$ \\
\hline 10 & $\begin{array}{l}\text { NEpi } \\
\text { KCN }\end{array}$ & $\begin{array}{l}2.0 \\
2.2\end{array}$ & $\begin{array}{l}2.2 \\
3.3\end{array}$ & $\begin{array}{l}3.0 \\
6.5\end{array}$ & $\begin{array}{l}3.9 \\
9.0\end{array}$ & $\begin{array}{r}4.9 \\
12.0\end{array}$ & $\begin{array}{r}6.2 \\
13.0\end{array}$ & $\begin{array}{c}7.2 \\
-\end{array}$ & $\begin{array}{c}7.5 \\
-\end{array}$ \\
\hline 11 & $\begin{array}{l}\text { NEpi } \\
\text { KCN }\end{array}$ & $\begin{array}{l}.2 \\
.6\end{array}$ & $\begin{array}{l}.5 \\
.9\end{array}$ & $\begin{array}{l}1.0 \\
2.7\end{array}$ & $\begin{array}{l}1.6 \\
4.0\end{array}$ & $\begin{array}{l}2.1 \\
5.5\end{array}$ & $\begin{array}{l}2.8 \\
7.3\end{array}$ & $\begin{array}{l}3.1 \\
9.0\end{array}$ & $\begin{array}{r}2.6 \\
15.0\end{array}$ \\
\hline 12 & $\begin{array}{l}\text { NEpi } \\
\text { KCN }\end{array}$ & $\begin{array}{r}1.3 \\
.8\end{array}$ & $\begin{array}{l}1.7 \\
1.8\end{array}$ & $\begin{array}{l}2.2 \\
3.0\end{array}$ & $\begin{array}{l}2.5 \\
7.1\end{array}$ & $\begin{array}{l}4.3 \\
8.0\end{array}$ & $\begin{array}{l}5.6 \\
9.0\end{array}$ & $\begin{array}{c}6.7 \\
-\end{array}$ & $\begin{array}{c}6.5 \\
-\end{array}$ \\
\hline 14 & $\begin{array}{l}\text { NEpi } \\
\text { KCN }\end{array}$ & $\begin{array}{l}2.5 \\
2.6\end{array}$ & $\begin{array}{l}2.5 \\
4.0\end{array}$ & $\begin{array}{l}3.0 \\
5.3\end{array}$ & $\begin{array}{l}4.2 \\
5.4\end{array}$ & $\begin{array}{l}5.3 \\
7.0\end{array}$ & $\begin{array}{r}4.8 \\
13.0\end{array}$ & $\begin{array}{r}5.5 \\
13.0\end{array}$ & $\begin{array}{c}7.0 \\
-\end{array}$ \\
\hline 17 & $\begin{array}{l}\text { NEpi } \\
\text { KCN }\end{array}$ & $\begin{array}{l}1.8 \\
1.6\end{array}$ & $\begin{array}{l}4.0 \\
4.0\end{array}$ & $\begin{array}{r}4.0 \\
12.0\end{array}$ & $\begin{array}{r}4.0 \\
12.0\end{array}$ & $\begin{array}{r}7.7 \\
20.0\end{array}$ & $\begin{array}{l}11.0 \\
29.0\end{array}$ & $\begin{array}{l}15.0 \\
29\end{array}$ & $\begin{array}{l}27.0 \\
30.0\end{array}$ \\
\hline 18 & $\begin{array}{l}\text { NEpi } \\
\text { KCN }\end{array}$ & $\begin{array}{l}1.3 \\
1.2\end{array}$ & $\begin{array}{l}3.8 \\
2.7\end{array}$ & $\begin{array}{l}3.8 \\
3.2\end{array}$ & $\begin{array}{l}6.6 \\
8.2\end{array}$ & $\begin{array}{r}6.6 \\
13.0\end{array}$ & $\begin{array}{r}6.5 \\
13.0\end{array}$ & $\begin{array}{l}10.2 \\
31.0\end{array}$ & $\begin{array}{l}12.0 \\
31.0\end{array}$ \\
\hline
\end{tabular}


FIGURE 17E

ARTERY i: PRESSURE-ELASTIC MODULUS CURVES FOR DISTENTION BEHAVIOR

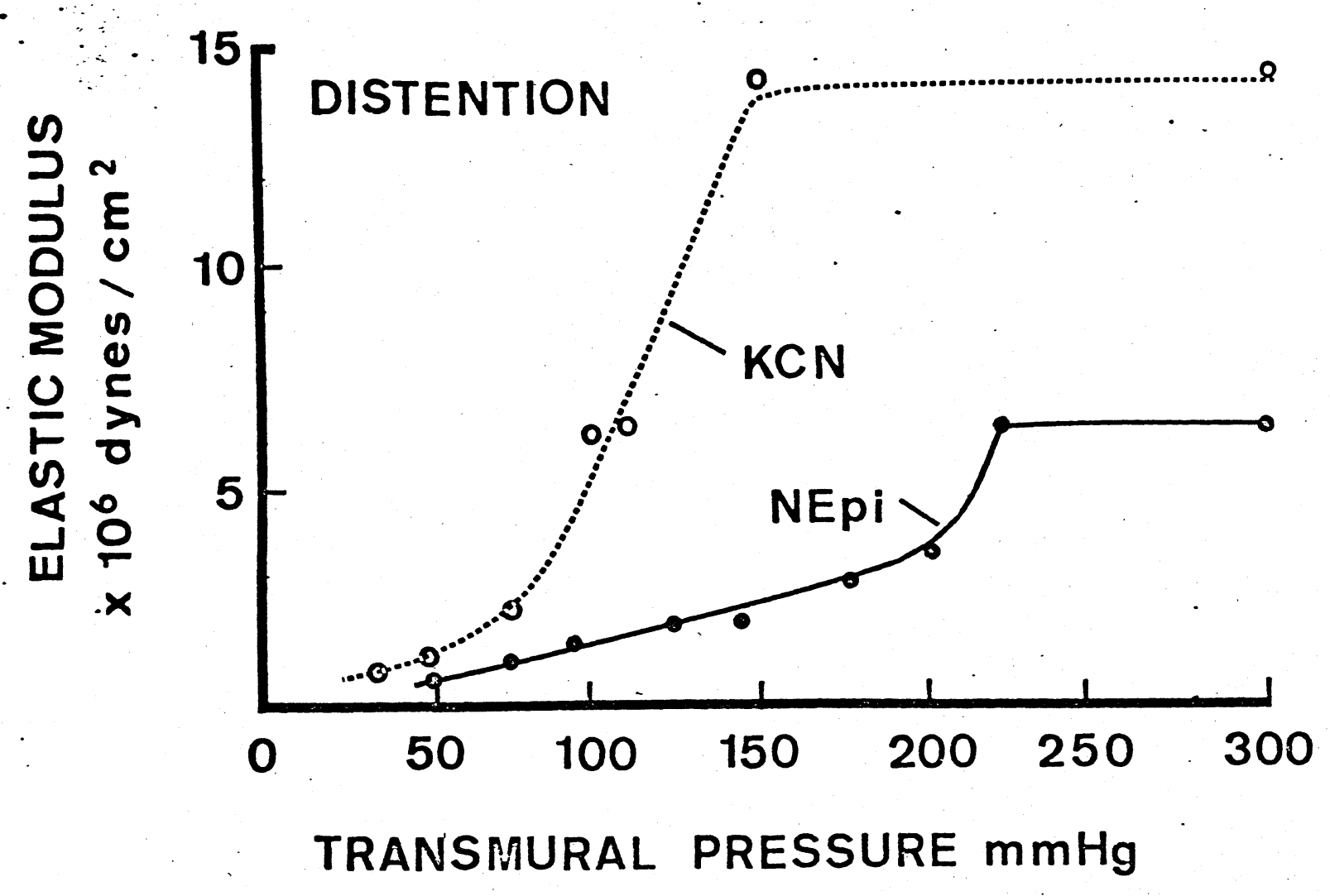




\section{FIGURE 18E}

ARTERY 2: PRESSURE-ELASTIC MODULUS CURVES FOR DISTENTION BEHAVIOR

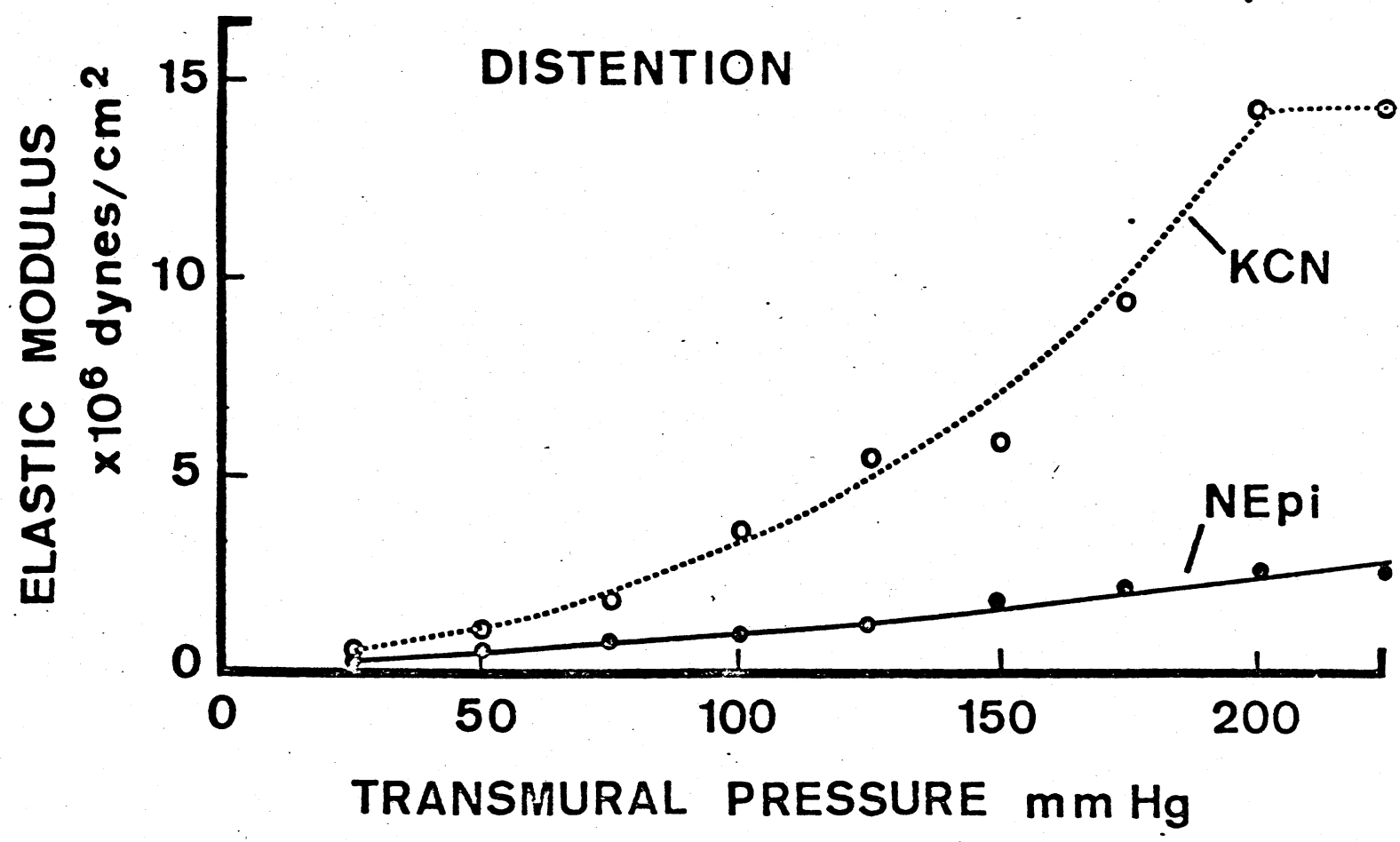


very different strains are associated with each pressure, depending upon whether or not the smooth muscie was active. The arrows in figures 170 and 180 indicate the strains associated with $100 \mathrm{~mm} \mathrm{Hg}$ after treatment with NEpi and after poinsoning with cyanide. Clearly NEpi decreases the strain at physlologi-. cal pressures, and also decreases the elasticity.

Results for 12 arteries are presented in Table IIIB. Some of these arteries were those subjected to equilibrium steps in both the shortening and distention sequences. These vessels also appear in Table II. The remaining arteries were those subjected to incomplete equilibrium steps during the distention sequences. In spite of considerable variability in the elasticity and maximum strain that was attained, every individual artery described in Table IIIA and IIIB agrees, qualitatively, with the two arteries analyzed in detall in Figures $17 A-E$ and $18 A-E$. In every artery studied, activation of smooth muscle caused contraction and rendered the arteries less distensible with respect to strain, but more distensible with respect to pressure. Figure 19 presents the mean elastic moduli and their standard errors plotted as a function of radius. It is clear that the elastic moduli gradually increase with increasing pressures, and that NEpi decreases the elasticity at all but the lowest pressures. The elastic moduli after NEPI and KCN were compared uging the Wilcoxon matched-palrs signed-ranks test (Siegel, 1956). 
MEAN ELASTIC MODULUS AND STANDARD ERRORS AS A FUNCTION OF PRESSURE; EFFECTS OF NEPI AND KCN ARE COMPARED WITH THE UNTREATED ARTERIES OF BERGEL.

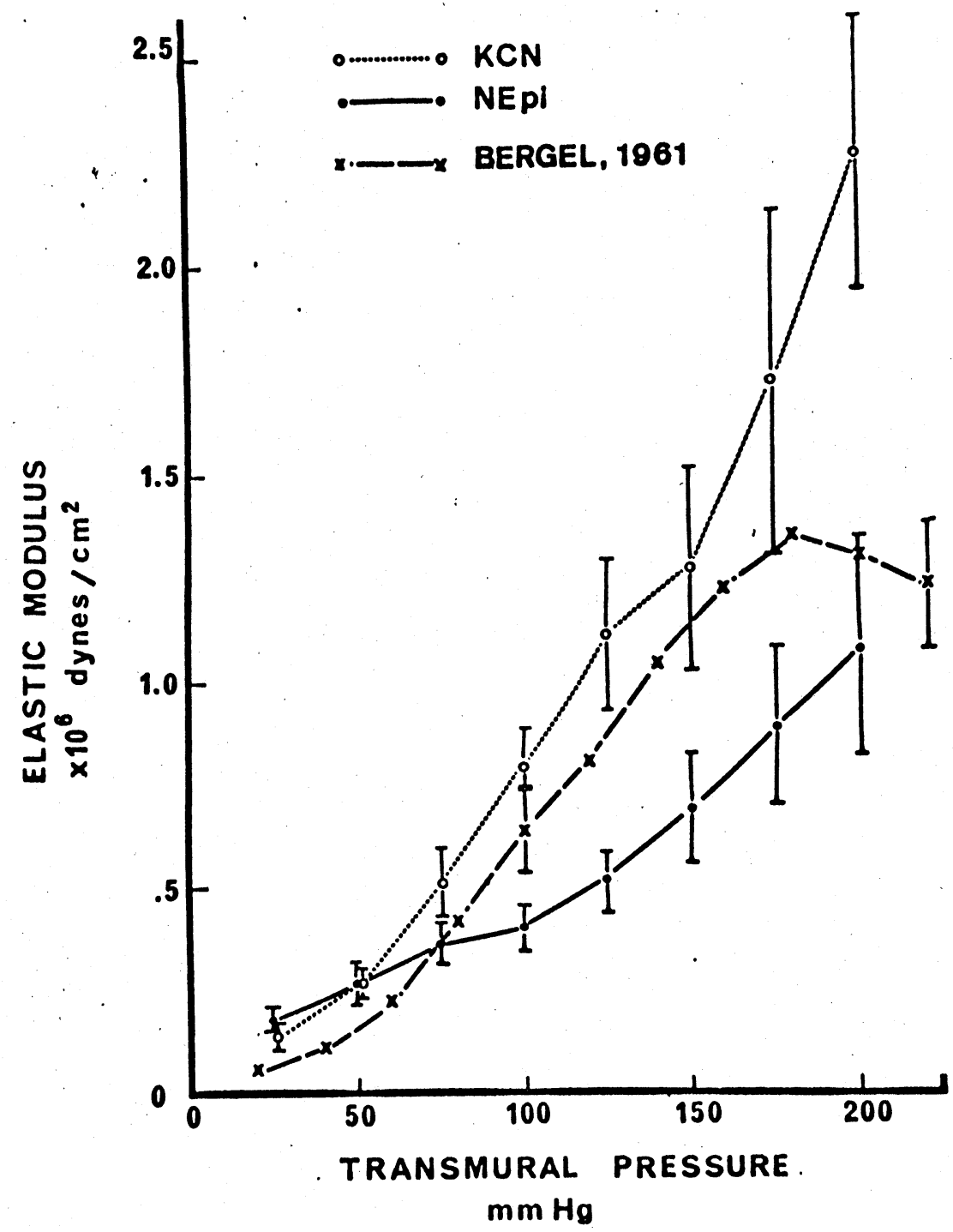


The differences at 25 and $50 \mathrm{~mm} \mathrm{Hg}$ were found to be insignificant, but the differences at all higher pressures were significant $(p<.025)$.

E. Hysteresis. 1. Interrupted Loops. It was noted above that treatment with NEpi markedly increases the hysteresis of arteries subjected to wide-range pressure-radius cycles. In order to investigate the properties of the loop, 4 arteries were subjected to interruption of the distention limb. Figure 20 illustrates one such experiment. After treatment with NEpi, the distention limb was interrupted first at a low pressure, and then during a second distention sequence, at a higher pressure. It was also interrupted at several pressures during distention sequences after poisoning with cyanide. Pressureradius curves after treatment with NEpi are plotted with filled circles and solid curves, after poisoning with KCN with open circles and broken curves, and during the shortening sequences following interruption with $x$ 's and widely broken curves. The shortening sequences following interruption tended to pass between the limbs of the hysteresis loop. After KCN the shortening and distention curves could almost be superimposed, no matter where they were interrupted. However, after treatment with NEpi the hysteresis loop was usually quide wide. If interrupted at a low pressure, the subsequent shortening sequence curves downward on the first pressure decrement and eventually terminated at approximately the point of minimum pressure and radius. If interrupted at a high pressure, the subsequent 
shortening sequence tended to exhibit very little change in radius until the pressure had been decreased 50-75 $\mathrm{mm}$ Hg. This curve then gradually turned downward, terminating near the point of minimum pressure and radius.

2. Shortening from Multiple Origins. In order to evaluate this behavior in more detail, two additional groups of arteries were subjected to shortening and distention sequences originating from various, randomly-ordered initial radil. Figures $21 \mathrm{~A}$ and $22 \mathrm{~A}$ illustrate pressure-radius curves of 2 arteries subjected to shortening from each of these initial radii ("origins"). A striking feature of these curves is the influence of KCN and NEpi on the behavior of the vessel. Changing the origin in the cyanide-poisoned vessel resulted in a merging of the subsequent shortening curves, while changing the origin in the NEpi-treated vessel generated a family of shortening curves which all tended to merge at the low point in the pressure-radius cycle. When the pressure or radius of origin was high the associated shortening curves tended to remain fairly flat over a long range of pressure. Figures 218 and 228 present stress-strain data derived from the shortening curves illustrate in Figures $21 \mathrm{~A}$ and $22 \mathrm{~A}$. Subtraction of the $\mathrm{KCN}$ curve from the NEpi curves indicates that the muscle exhibits a graded contractile stress. At each strain, the shortening curves originating from a low origin exhibits more active stress than the 


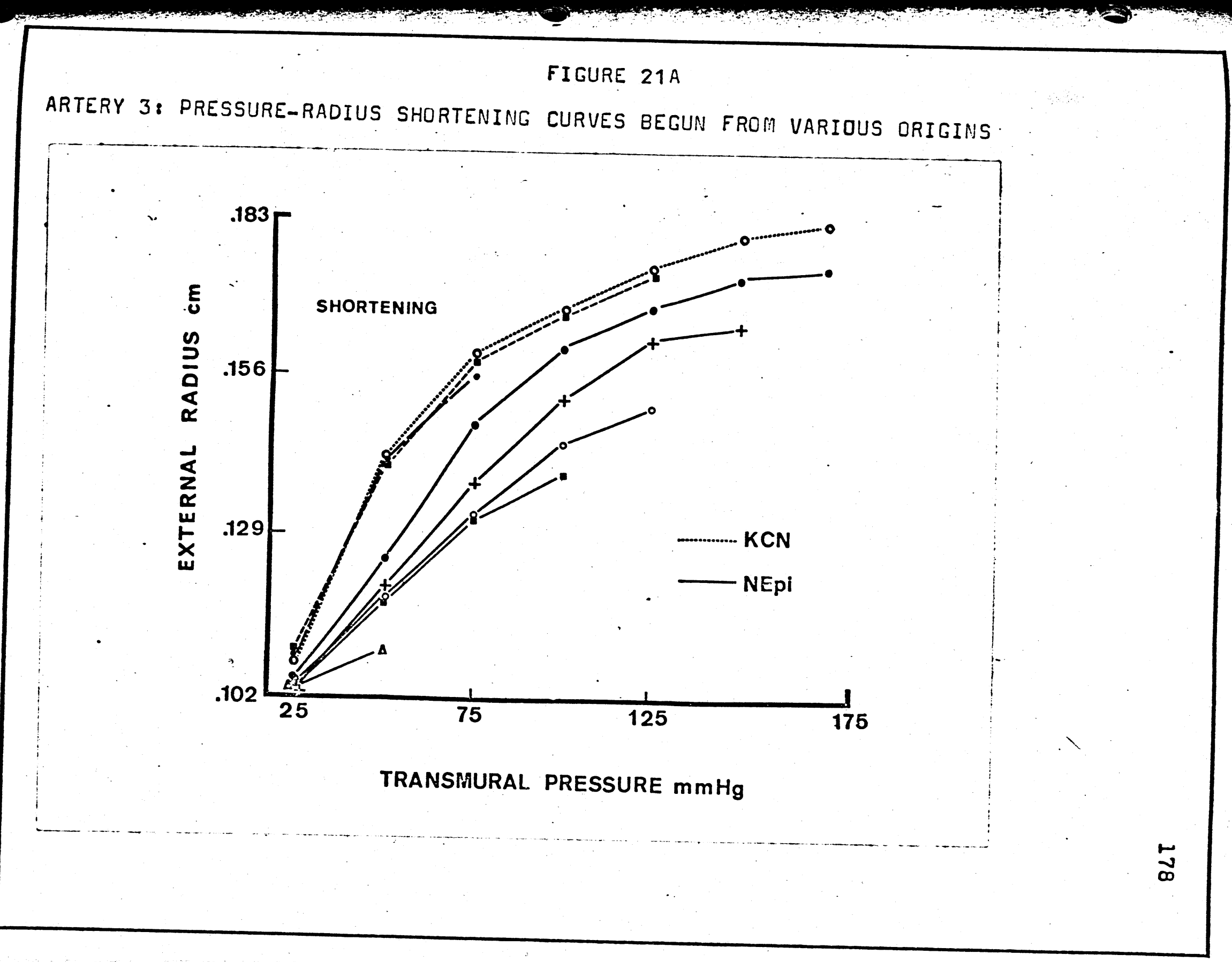


FIGURE 218

ARTERY 3: STRESS-STRAIN CURVES FOR SHORTENING SEQUENCES BEGUN FROM VARIOUS ORIGINS

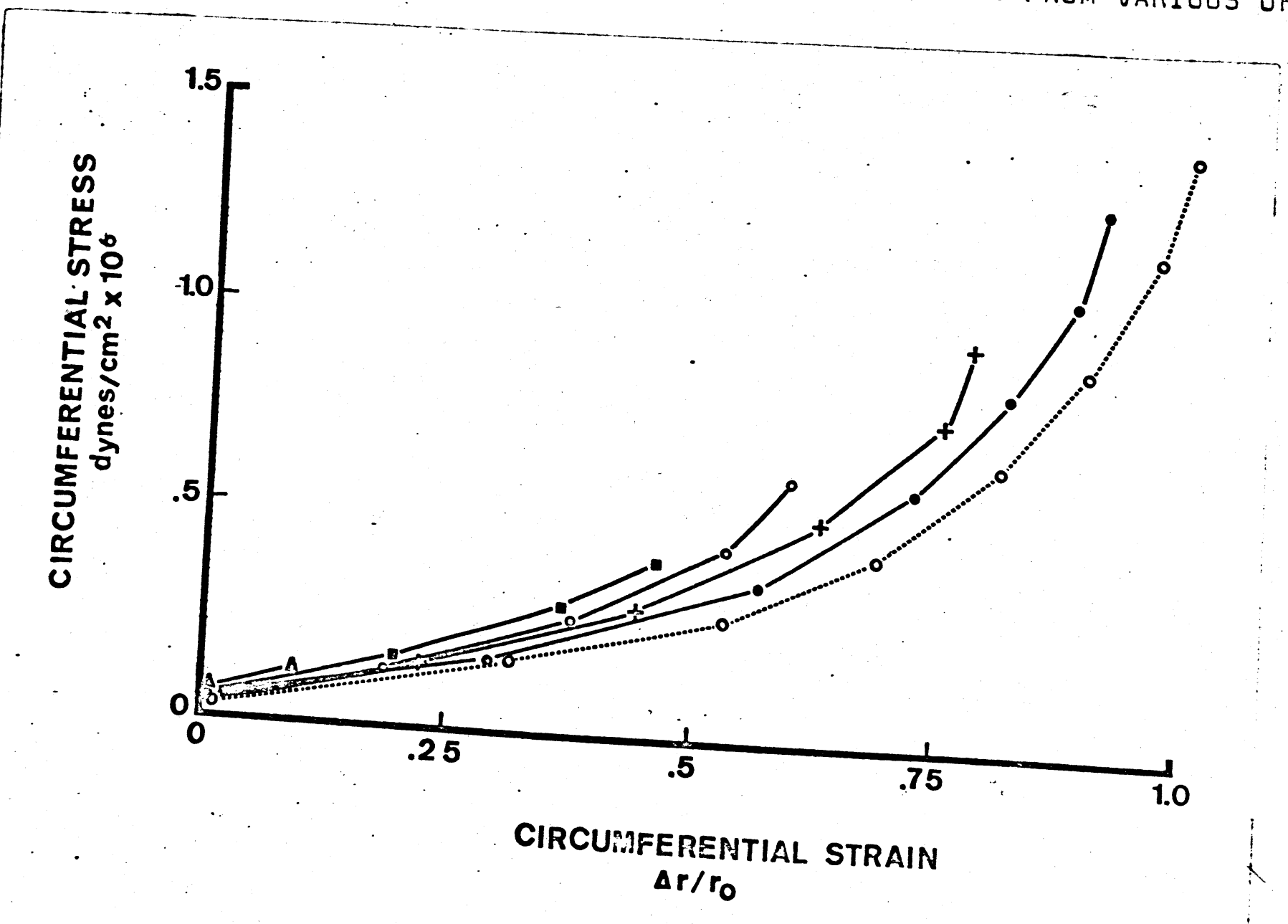




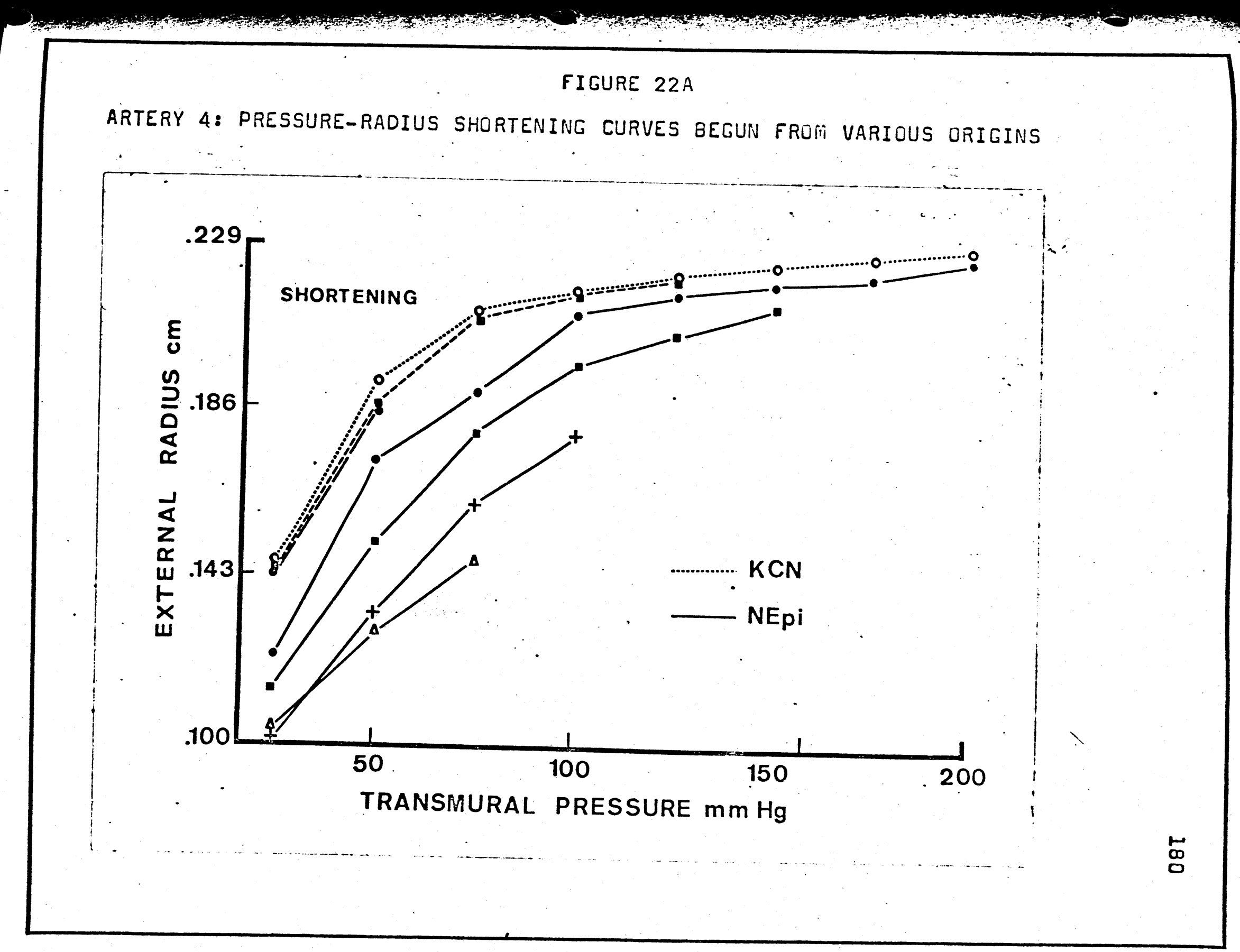


ARTERY 4: STRESS-STRAIN CURVES FOR SHORTENING SEQUENCES BEGUN FROM VARIOUS ORIGINS

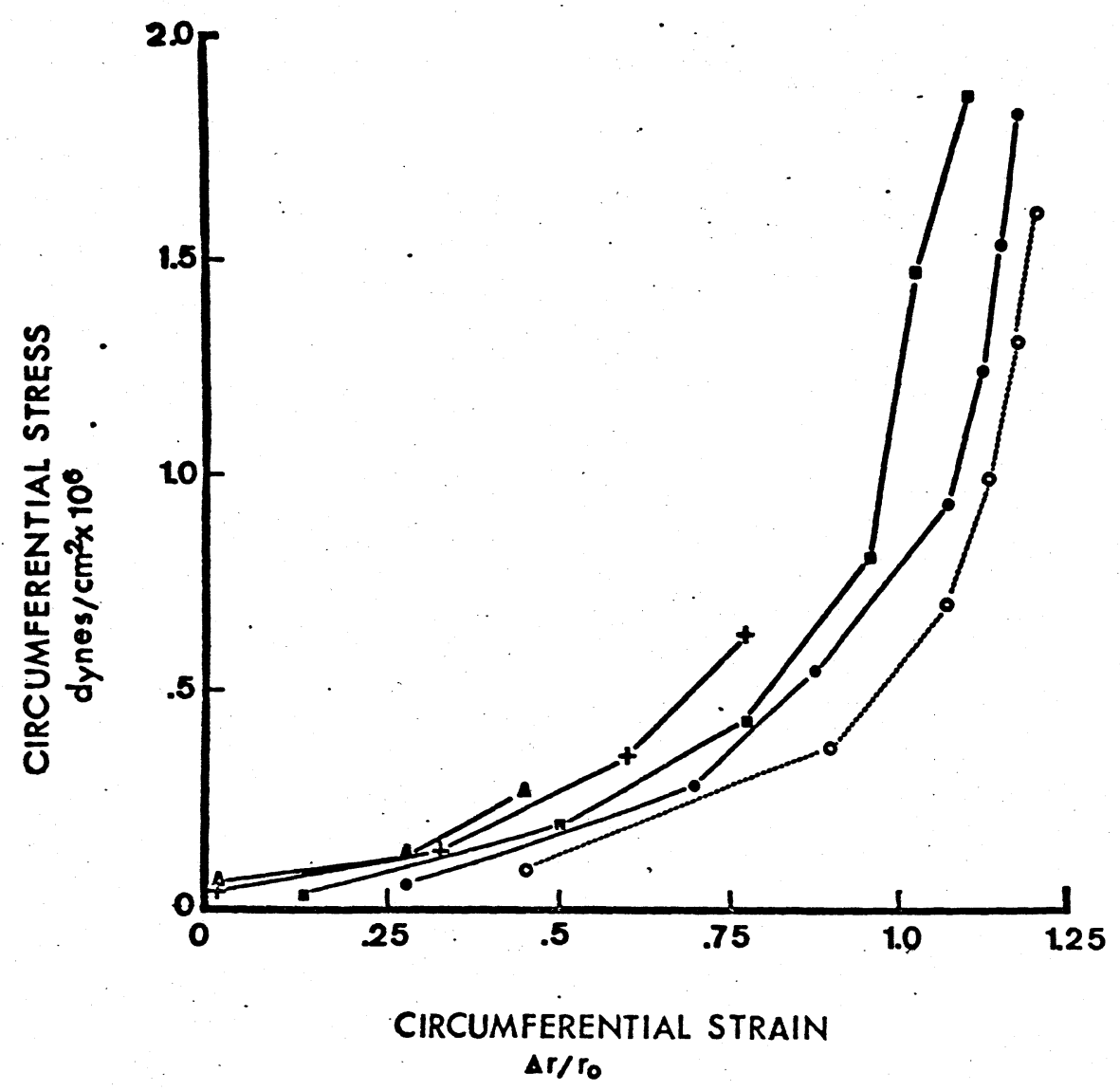


shortening curves originating from successively higher origins. Similar behavior was observed in 9 separate arteries.

3. Distention from Multiple Origins. Figures $23 \mathrm{~A}$ and $24 \mathrm{~A}$ present pressure-radius curves of arteries subjected to distention from multiple, randomly-ordered origins. All of the pressure-radius curves after poisoning with cyanide are strikingly superimposed, while those obtained when the smooth muscle was activated with NEpi describe a family of curves. When the NEpi-treated vessel originated from a small initial radius it tended to remain smaller throughout the distention range than when it originated from a larger initial radius. Although these curves originated from different radii and traversed separate paths, they all converged at about $250 \mathrm{~mm} \mathrm{Hg}$. Figures 238 and 24 B present stress-strain data derived from the previous pressure-radius curves illustrated in Figures $22 \mathrm{~A}$ and $23 \mathrm{~A}$. Seventyfive percent of the slope of the stress-strain curves was determined, and these data are presented in Figures $24 \mathrm{C}$ and $25 \mathrm{C}$ in graphs of the elastic moduli as a function of strain. Figures $23 \mathrm{C}$ and $24 \mathrm{C}$ indicate that when an artery originates from a small radius and low pressure it tends to exhibit a lower elastic modulus, at any given strain, than when it originates from a larger radius and higher pressure. The fact that arteries originating from both small and large radii (Figures $23 \mathrm{~A}$ and $24 \mathrm{~A}$ ) ultimately converge at high pressure and radii indicates that this gradation 
FIGURE $23 \mathrm{~A}$

ARTERY $5 \&$ PRESSURE-RADIUS DISTENTION CURVES BEGUN FROM VARIOUS ORIGINS

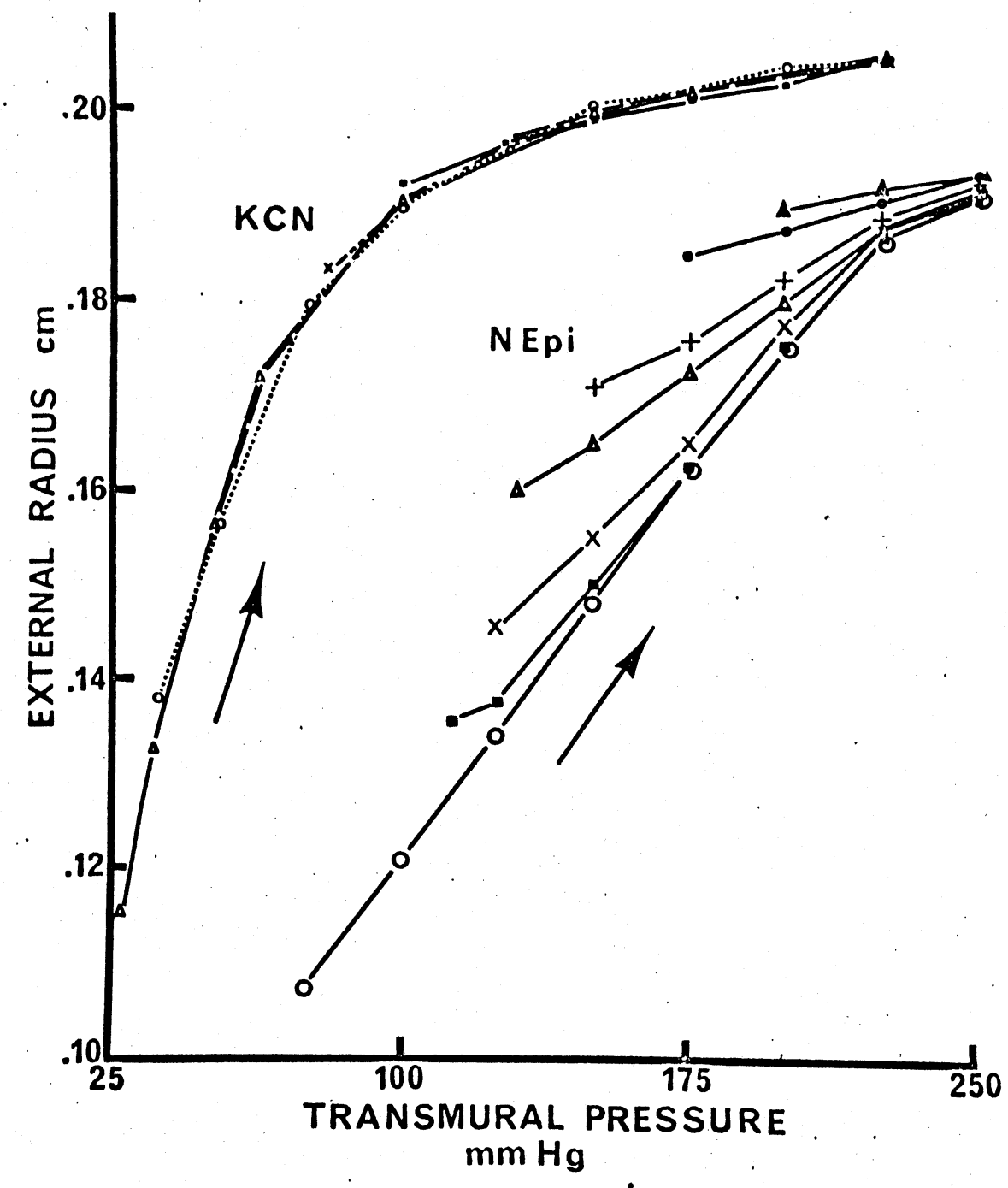




\section{FIGURE 24A}

ARTERY 5: PRESSURE-RADIUS DISTENTION CURVES BEGUN FROM VARIOUS ORIGINS

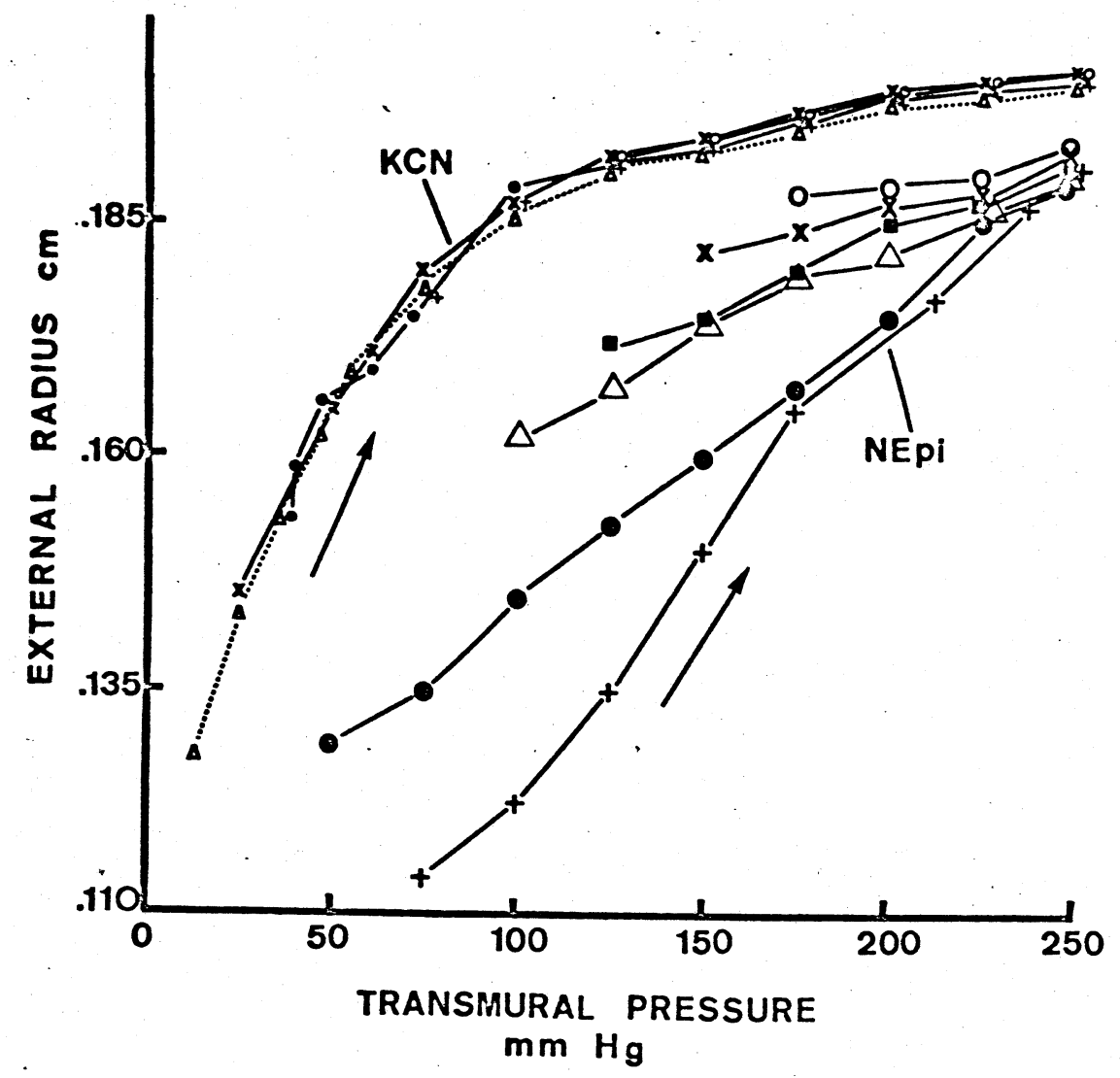


FIGURE 238

ARTERY $5:$ STRESS-STRAIN CURVES FOR DISTENTION

SEQUENCES BEGUN FROM VARIOUS ORIGINS

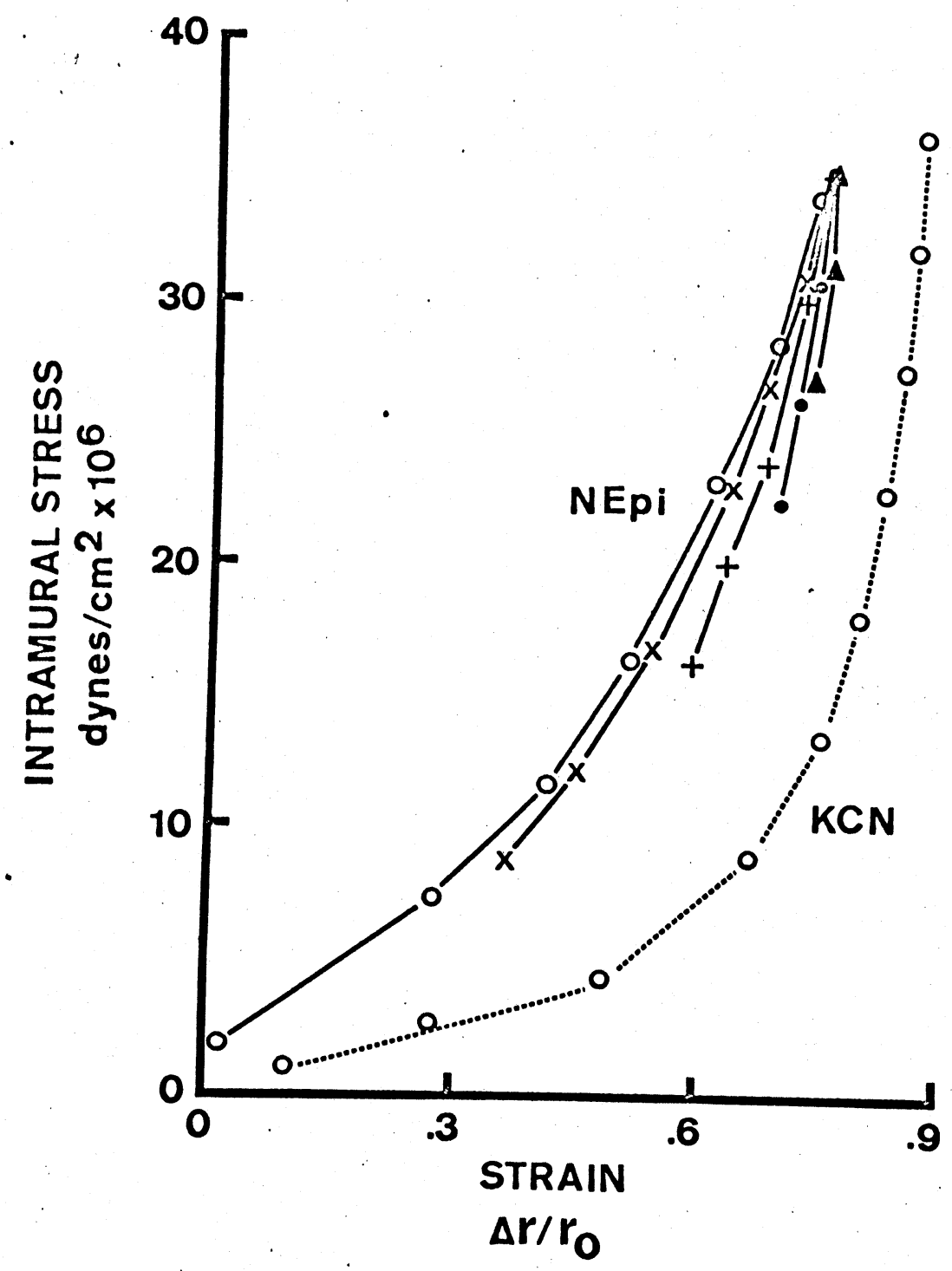


ARTERY 6: STRESS-STRAIN CURVES FOR DISTENTION SEQUENCES BEGUN FROM VARIOUS ORIGINS

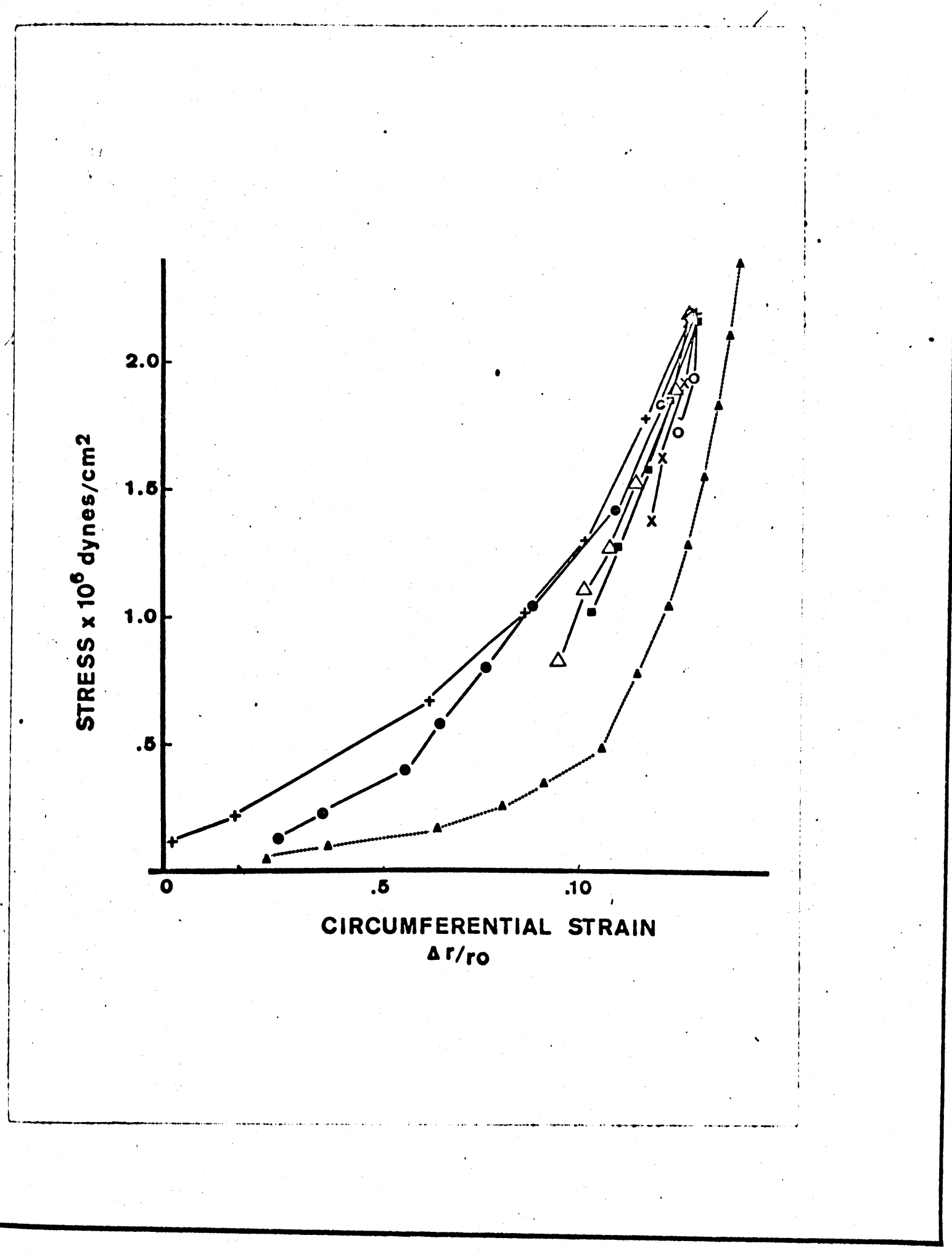


FICURE 23C

ARTERY 5: STRAIN-ELASTIC MODULI CURVES FOR DISTENTION SEQUENCES BEGUN FROM VARIOUS ORIGINS

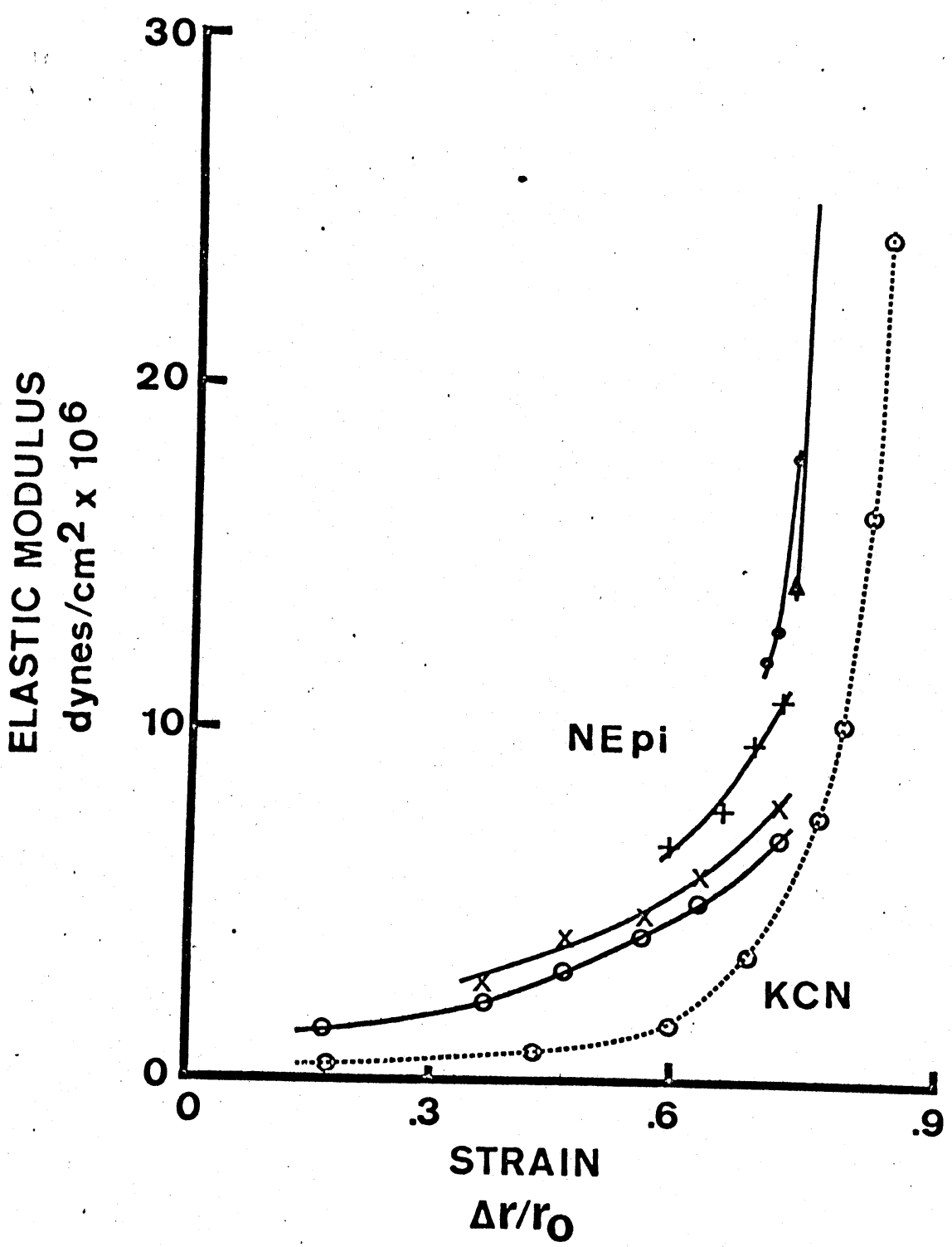

$\because$ 
ARTERY 6: STRAIN-ELASTIC MODULI CURVES FOR DISTENTION SEQUENCES BEGUN FROM. VARIOUS ORIGINS

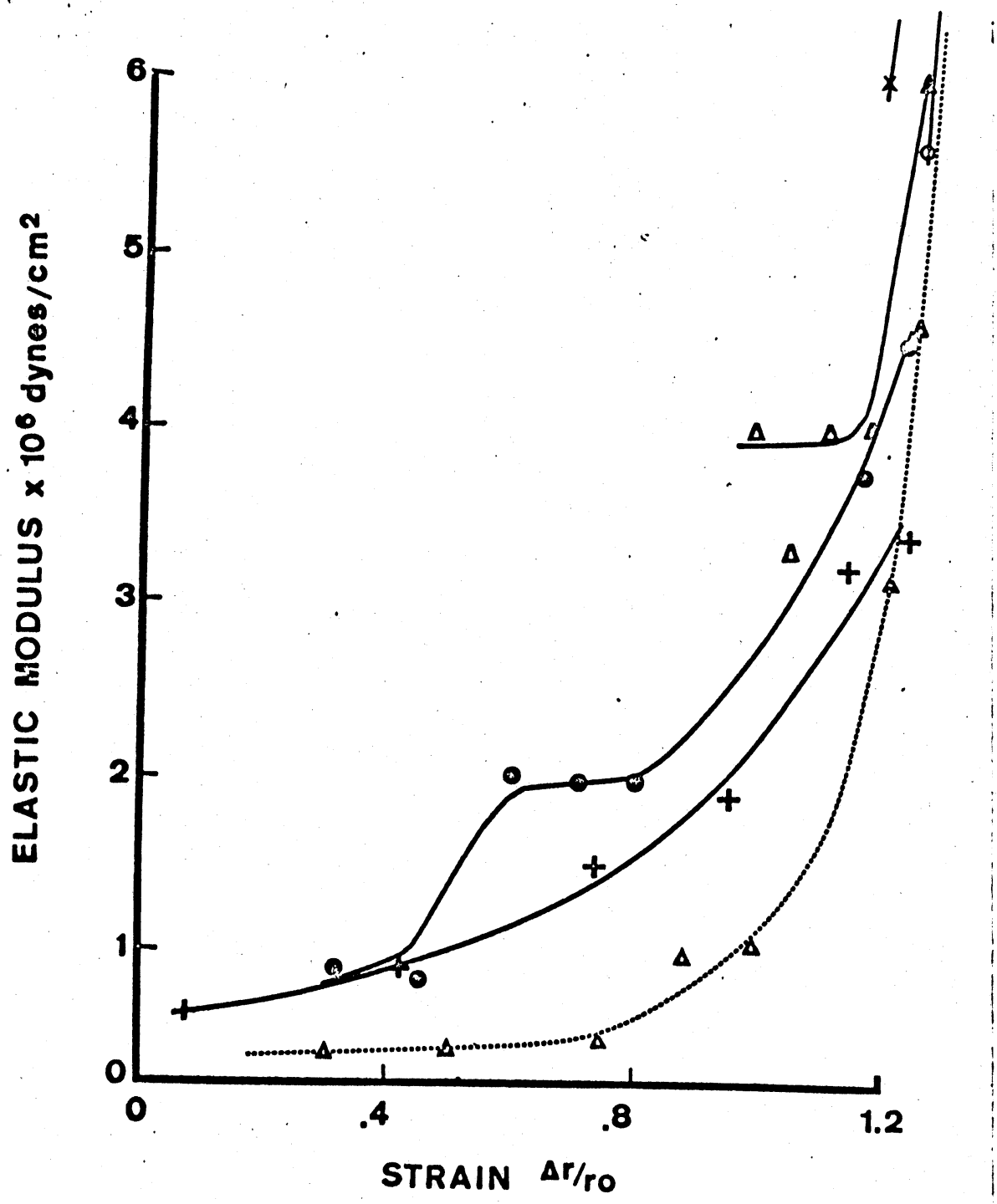


of elastic moduli must exist because a small vessel must be more distensible than a large vessel if they are both to pass through identical pressure-radius coordinates. This behavior was observed in experiments on 8 separate arteries. Thus, for both shortening and distention, the properties of an active artery, at any given radius, depend upon its previous dimension. The fact that this does not apply to cyanide-poisoned vessels indicates the essential role of active muscle.

The shortening sequences (Figures $21 \mathrm{~A}$ and $22 \mathrm{~A}$ ) each represent one-half of a set of pressure-radius hysteresis loops. Figures 218 and 228,238 and 248 are the stress-strain counterparts. The missing half of the hysteresis loops may be envisioned by joining together the points of origin on any one figure by an imaginary distention or shortening line. It is evident from these data that the width of the loop in the NEpitreated vessel depends upon the magnitude of the radius change. A small change in radius produces a small loop, and larger changes in radius produce progressively larger, enveloping loops F. Longitudinal Properties. Figure 25 presents two segments of Polygraph records illustrating an experiment performed on an in situ-length artery. In the upper (A) panel, the vessel was untreated. Each decrease in pressure $\left(P_{t}\right)$ was accompanied by an increase in the longitudinal tethering force 
FIGURE 25

POLYGRAPH RECORDS ILLUSTRATING LONGITUDINAL FORCE MEASUREMENTS
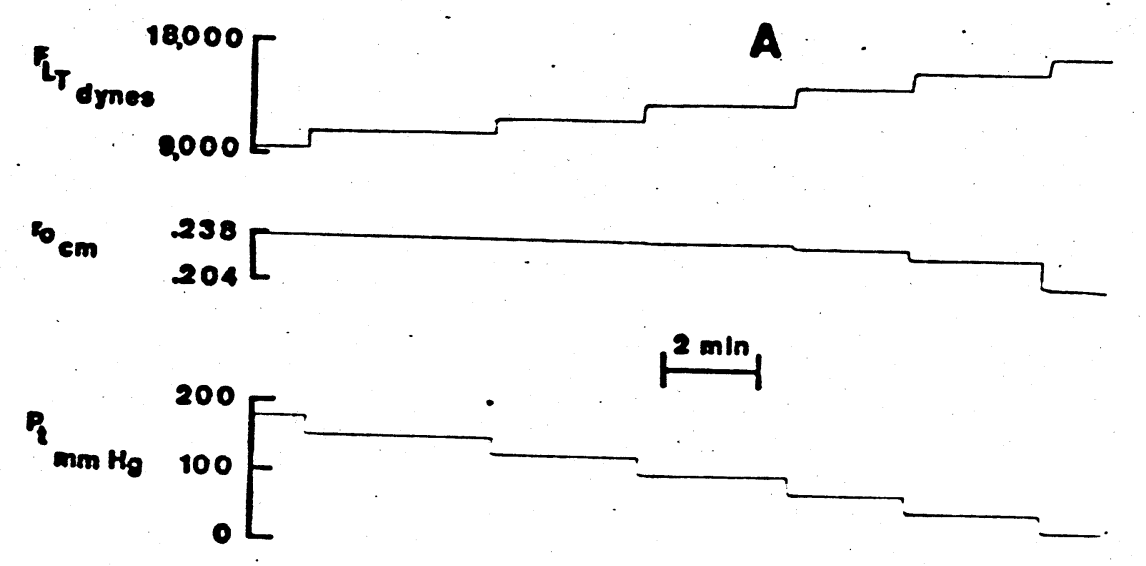

B

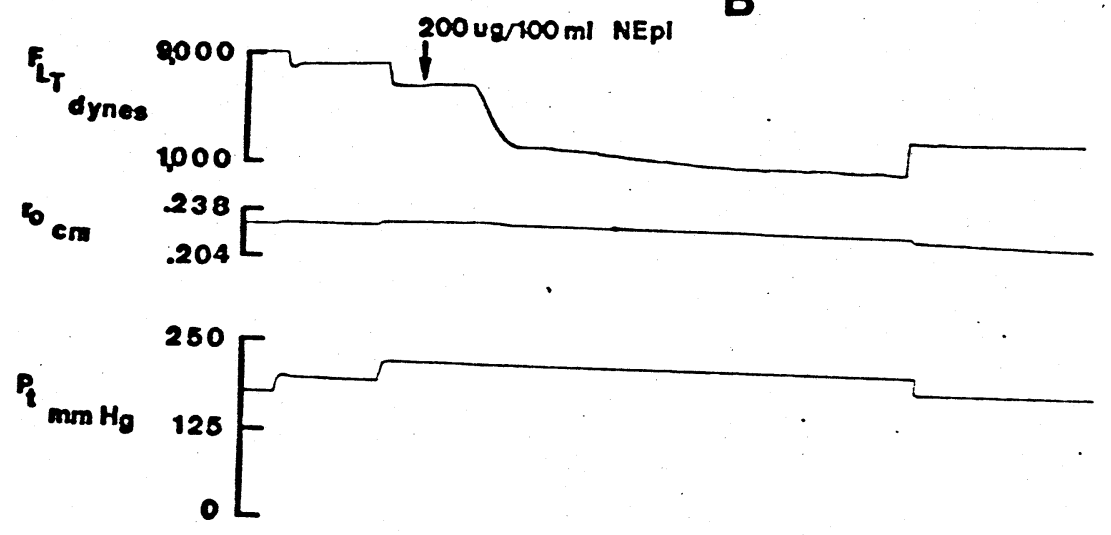

A. VESSEL IN PRETREATMENT CONDITION. NOTE INCREASE IN LONGITUDINAL FORCE WITH EACH PRESSURE DECREASE, WHETHER OR NOT THERE IS A CHANGE IN RADIUS.

B. EFFECTS OF KEPI. NOTE FALL IN LONGITUDINAL FORCE WITH NO CHANGE IN PRESSURE AND NEGLIGIBLE CHANGE IN RADIUS.

மே 
$\left(F_{L T}\right)$ that was measured by the force transducer. This occurred whether or not there was a change in radius. In the lower (B) panel, the vessel was treated with $200 \mathrm{\mu g} / 100 \mathrm{ml} N E p i$ at the arrow. Such treatment caused a striking decrease in the longitudinal-tether force with almost no change in radius. The direction and magnitude of changes in tethering force which are associated with step changes in pressure before treatment with NEpi indicate that the decrease in longitudinal tether which appears soon after treatment with NEpi could not result from extremely small, undetected changes in radius. Pressure is held constant by the regulating system. Thus, the decline in longitudinal tether appears to result from some direct action of NEpi upon the wall under essentially isometric conditions.' Similar results were observed in 5 experiments.

Under conditions of zero transmural pressure, the in situ length vessel exerts a constant, retractive longitudinal force. This may be termed the force of initial tether. The initial tether reflects the force necessary to maintain the in situ length of the unpressurized, undistended artery. It also reduces the radius to a smaller value than would exist if the vessel were unextended. Circumferential stress and strain valves, discussed earlier, were computed using the small radius encountered in the extended artery as zero strain. Similarly, 
longitudinal stresses may be calculated by referring to the initial tether as the functional zero baseline.

Longitudinal stress was calculated as the sum of the stress due to pressure and the stress due to the alterations in tether above or below the force of initial tether:

$$
\sigma_{L}=\sigma_{L_{P}}+\Delta_{L_{T}}
$$

Stress due to pressure was calculated according to equation 8b,

$$
\sigma_{L_{P}}=\frac{P_{T} \times r_{i}{ }^{2}}{r_{0}{ }^{2}-r_{i}{ }^{2}}
$$

and the stress due to changes in tether was calculated by dividing the net tether force by the cross-sectional area of the walf,

$$
\Delta \sigma_{L_{T}}=\frac{F_{L_{T}}-F_{i T}}{\pi\left(r_{0}{ }^{2}-r_{i}{ }^{2}\right)}
$$

The stress due to pressure is positive at all radii, and the stress due to alterations in tether is negative over the entire radius range. Figures $26 \mathrm{~A}$ and $27 \mathrm{~A}$ present the longitudinal stresses as a function of circumferential strain. It is clear that, in spite of the fact that NEpi decreases the tether component, NEpi increases the net longitudinal stress at any given circumferential strain. This is because the increase in the pressure component associated with NEpi is greater than tho decrease in the tether component. Longitudinal strain is 
ARTERY 7: LONGITUDINAL STRESS-CIRCUMFERENTIAL STRAIN CURVE.

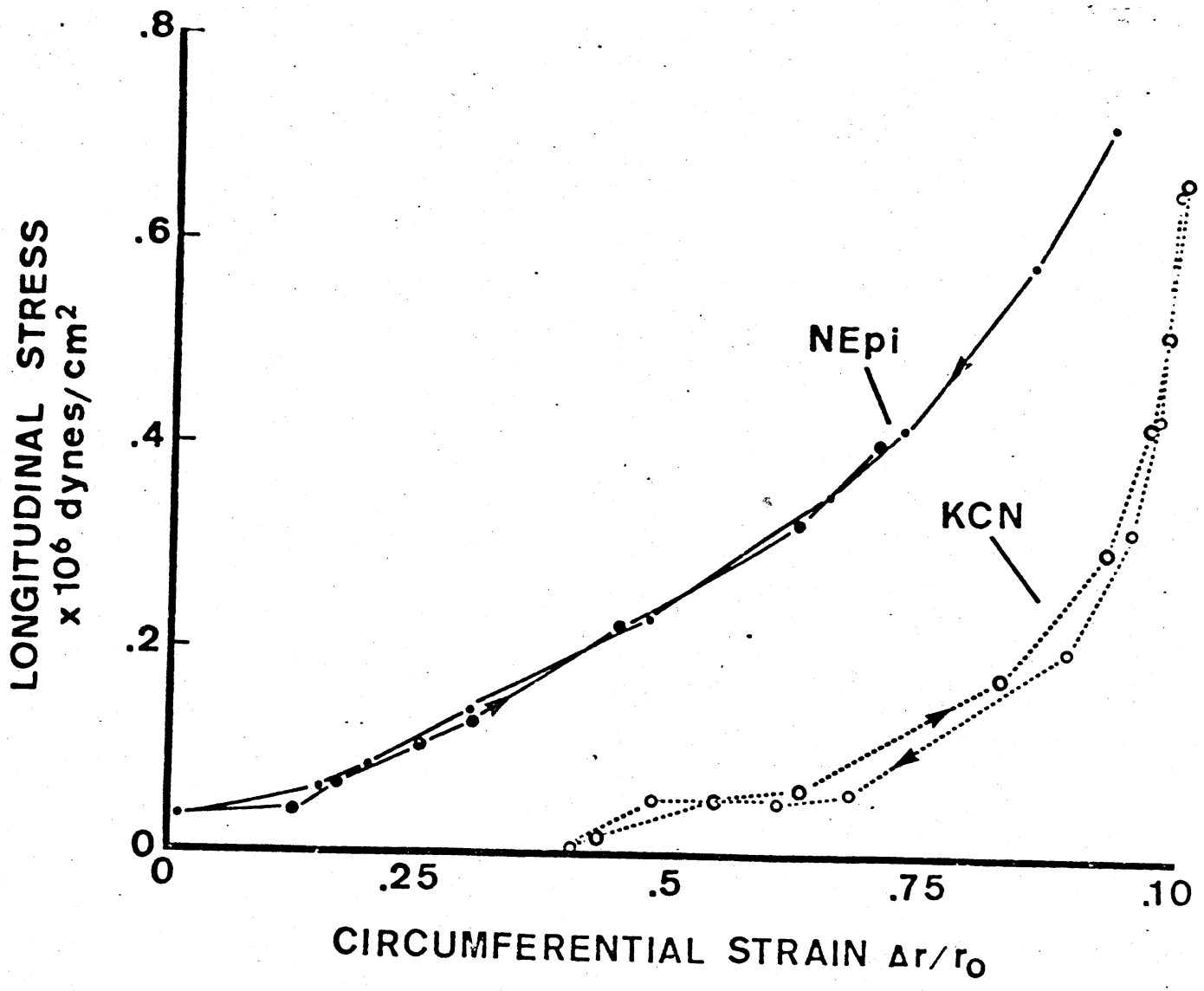


ARTERY 8: LONGITUDINAL STRESS-CIRCUMFERENTIAL STRAIN CURVE.

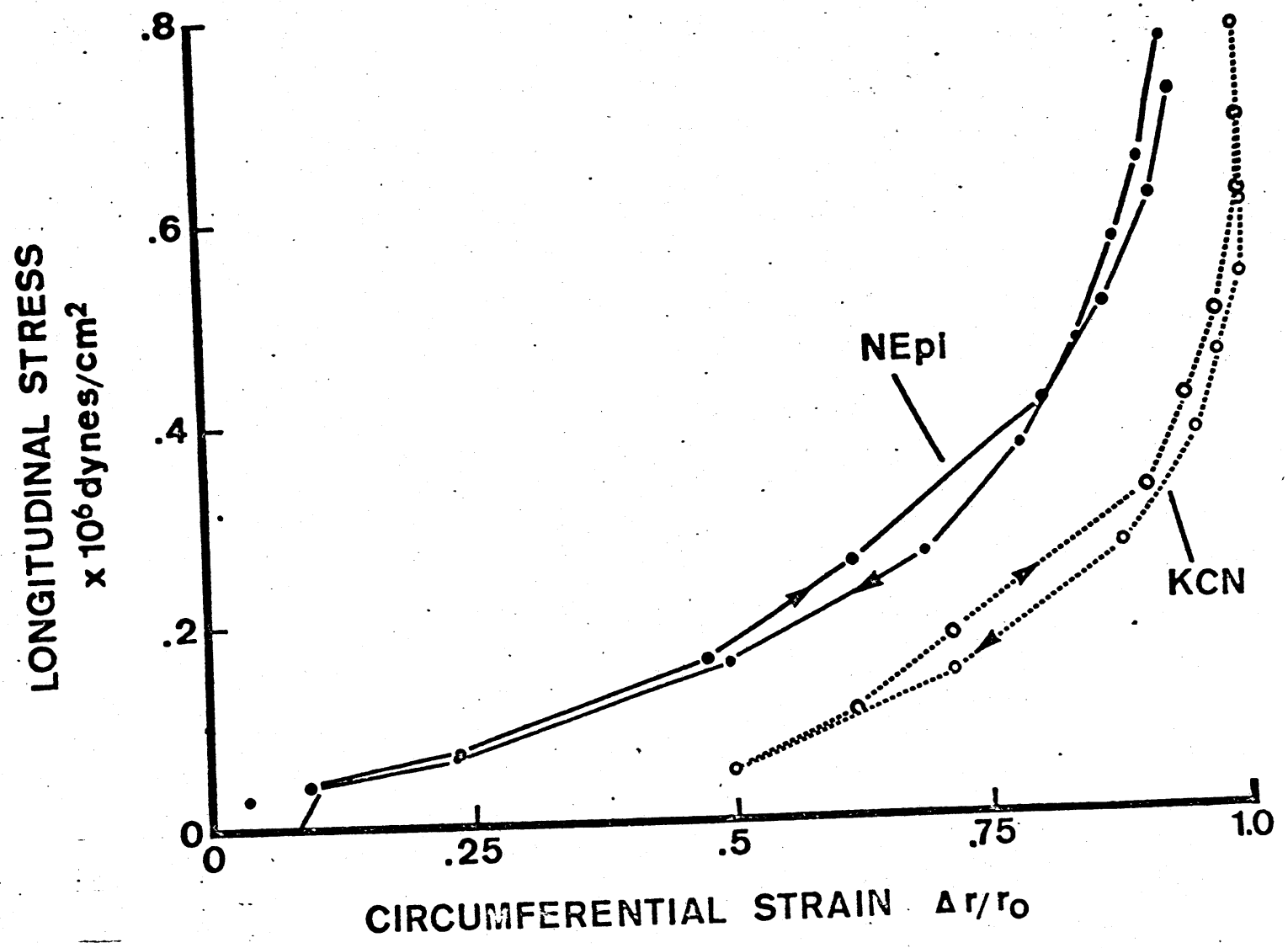


essentially fixed in these experiments. It is also clear that following treatment with either NEpi or KCN the longitudinal stress increases with increasing radius. This is contrary to the unchanging length predicted of an isotropic tube undergoing circumferential expansion. by Equation 20. It is apparent that the behavior of the arterlal wall, when elongated to in situ length is anisotropic.

It was of interest to estimate the extent to which the wall deviated from isotropy. Equation 20 related circumferential and longitudinal properties for an isotropic material.

$$
\varepsilon_{L}=\frac{\sigma_{L}}{E_{L}}-\frac{\sigma_{C}}{E_{C}}
$$

where,

$$
\begin{aligned}
& \varepsilon_{L}=\text { longitudinal strain } \\
& \sigma_{L}=\text { longitudinal stress } \\
& E_{L}=\text { longitudinal elastic modulus } \\
& \nu=\text { Poisson's ratio } \\
& \sigma_{C}=\text { circumferential stress } \\
& { }_{C}=\text { circumferential elastic modulus }
\end{aligned}
$$

In an isotropic material Poisson's ratio is equal to 0.5 , and circumferential and longitudinal elastic moduli are equal. This equation was used as follows. Longitudinal strain was set equal to zero because the length of the arteries was 
essentially fixed. Circumferential stresses were calculated from circumferential stress-strain plots. The slopes of these curves were graphically determined, and $75 \%$ of these values were used as the circumferential elastic moduli. Poisson's ratio was taken as 0.5 , as indicated from the previous wall volume studies. Finally, the equation was solved for the longitudinal elastic moduli at each circumference. Figures 268 and 278 present graphs in which circumferential olastic moduli are plotted against longitudinal elastic moduli. The behavior of the arterlal wall would be termed isotropic only if the points fell along the line of equality. The curves indicate that the circumferential elastic modulus is consistently greater than the longitudinal elastic modulus. In one case the ratio, $E_{C}: E_{L}$, is about $1.3: 1$ after poisoning with $K C N$, and this is increased to about $2: 1$ when the smooth muscle is activated with NEpi. In the other case the ratio is 1.281 after poisoning with $K C N$ and this is increased to $1.4: 1$ when the smooth muscle is activated with NEpl. At very low values, the longitudinal modulus is equal to, or greater than, the circumferential modulus. 


\section{FIGURE 26B}

ARTERY 7: PLOT OF CIRCUMFERENTIAL ELASTICITY AGAINST LONGITUDINAL ELASTICITY

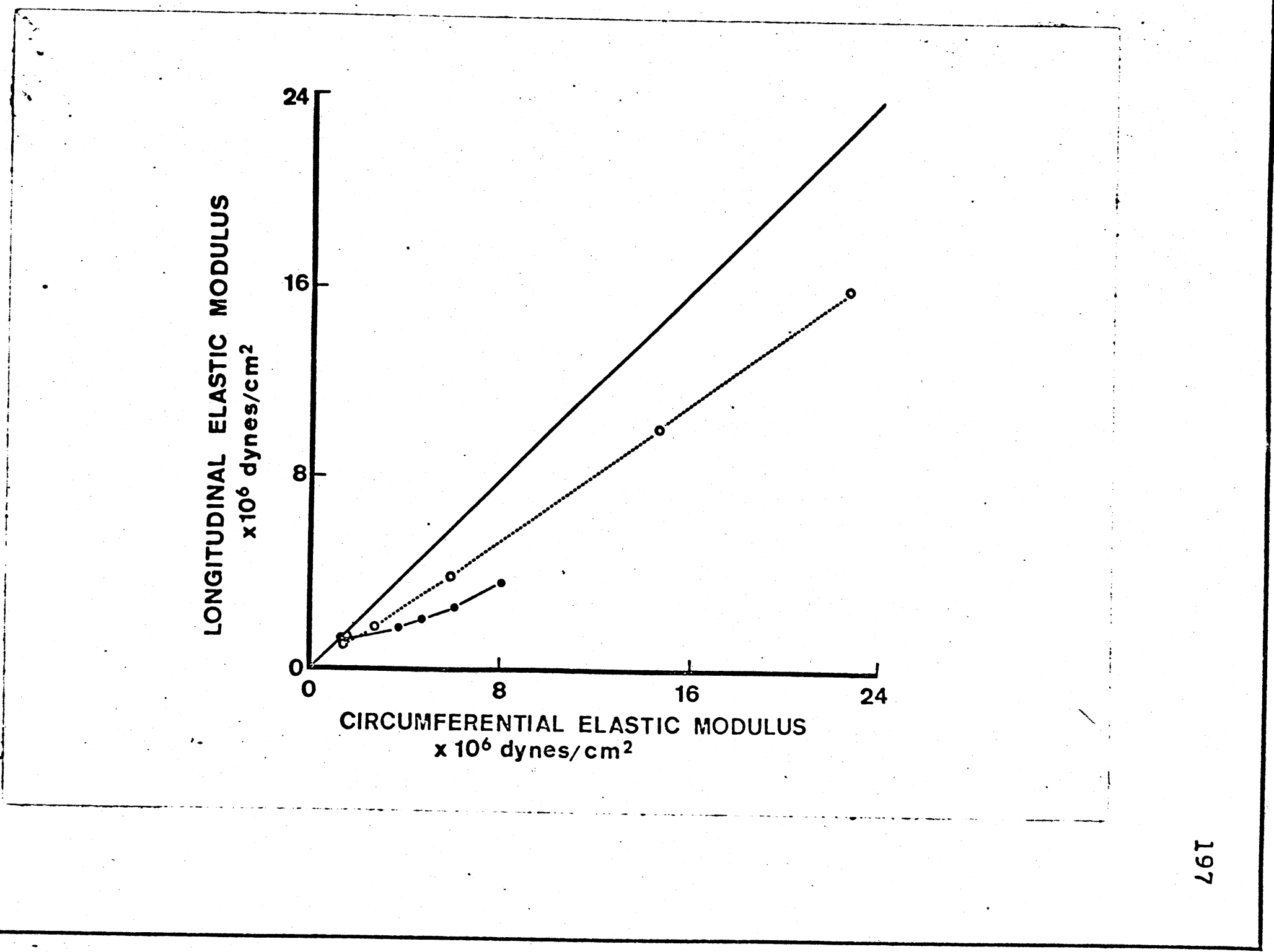


FIGURE 27B

ARTERY 8: PLOT OF CIRCUMFERENTIAL ELASTICITY AGAINST LONGITUDINAL ELASTICITY

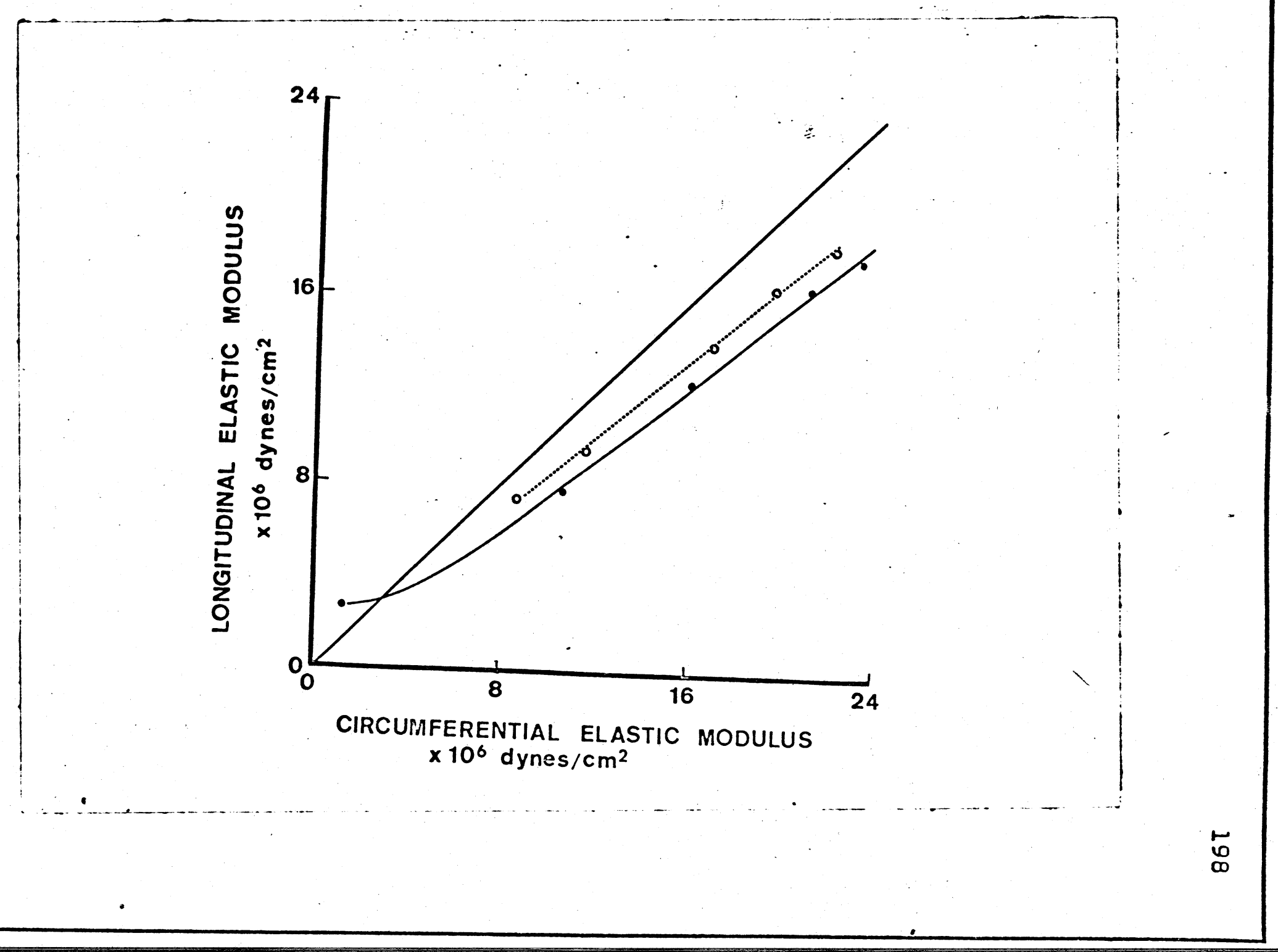




\section{CHAPTER V}

\section{DISCUSSION}

A. Wall Volume. The present results indicate that the volume of the arterial wall, in fixed-length preparations, remains constant over a wide range of physiological pressures. These may be compared with the findings of Lawton (1954), Fenn (1957), Tickner (1965), and Carew et al (1968). Lawton and Carew both studied arteries in fluid-filled chambers, sealed except for a capillary tube projecting vertically out of the top of the chamber. Changes in the level of the fluid in the capillary tube were measured to indicate changes in the volume of the arterial specimen. Water was assumed to be virtually incompressible. Lawton (1954) stretched aortic strips cut at transverse, longltudinal and diagonal angles. His data show changes in volume of $3 \%$ or less over the entire range of extensions studied. Carew mounted cylindrical segments of dog aorta in such a chamber, and transferred known volumes of fluid from the extravascular region of the chamber to the intralumenal space. Extremely undistensible tubing and leakproof fittings were used. The bulk modulus of the artery, calculated in this way was found to 
be $4.35 \times 10^{9}$ dynes $/ \mathrm{cm}^{2}$. This may be compared with a value for the bulk modulus of water of approximately $2.33 \times 10^{10} \mathrm{dynes} / \mathrm{cm}^{2}$ at atmospheric pressure and $100^{\circ} \mathrm{F}$ (Rouse, 1938). The findings of Carew et al indicate that the arterial wall is about only 5 times as compressible as water. However, it is well to note tha the arterial wall is also permeable to water. The imposition of a strong compressive force across the wall might cause an extravasation of water from the wall tissue into the surrounding fluid. The level of fluid in the capillary tube was used to measure changes in arterial wall volume, but this method does not really indicate the volume of the arterial wall; it really indicates the volume of the total contents of the chamber. A transfer of fluid from one compartment to another compartment within the same chamber would therefore go undetected. According to Harkness ot al (1957) and Fischer and Llaurado (1966), $70 \%$ of the arterial wall is water. If the volume of the specimen is small relative to the volume of the chamber, as it was in the experiments of Carew ot al, then it is no surprise that the bulk modulus of the wall, determined under thase conditions, approaches the bulk modulus of water. The results of the present experiments agree with the findings of Lawton and Carew, but were performed using less elegant, but somewhat less equivocal, methods. 
The experiments of Fenn (1957) and Tickner (1965) employed visual techniques. Fenn hung arteries vertically and perfused them from below with a saline-filled U-tube. The lower and of the artery was fixed and changes in length were determined by measuring the movement of the upper, ligated end of the artery. Intralumenal volume and length were determined at each pressure step, and the relationship between internal diameter and wall thickness was determined microscopically at the end of the experiment. Fenn found that when the pressure was increased, the artery expanded both circumferentially and longitudinally. Fenn felt that this indicated that the wall was undergoing compression, and he calculated Poisson's ratios of as low as 0.3. However, the experiments were performed on fully retracted arteries, and properties defined under these conditions may bear little resemblence to the extended in situ condition. If nothing else, one may anticipate apparent alterations in mechanical properties because of changes in position along the alinear circumferential and longitudinal stress-strain curves. Tickner (1965) also studied longitudinally extensible arteries by $x$-raying vertical, mercury-filled vessels. He found that the circumferential and longitudinal stiffnesses were equal, that radial stiffness was independent of both internal pressure and axial loading, and he, too, concluded that the arterlal wall behaves as a compressible material. In Tickner's 
experiments wall thicknesses were measured with an x-ray technique. A fow attempts were also made in the present experiments to measure wall volumes in extensible vessels. At short lengths the outer layers of the wall and remnants of the sheath wrinkled, producing an immeasureable "trapped volume"of air and fluid. The approach was therefore abandoned. Furthermore, the present experiments had the advantage of a definable lead oxide layer on the outer surface of the vessel, while Tickner's did not, making his measurements that much more uncertain. In any case it is possible that the wall is compressible when it is under stress in the retracted state, but this state never occurs in life. From the present experimental data on extended, in situlength vessels, the arterial wall appears to be essentially isovolumetric. This concurs with the findings of Lawton and Carew et al, but refutes the findings of Fenn and of Tickner. Only the studies of Carew at al were performed on vessels possessing a geometry comparable to those employed in the present experiments.

B. Actions of NEpi and KCN. NEpi causes arteries to constrict. This was observed at pressures of $150 \mathrm{~mm} \mathrm{Hg}$ or less in every artery studied, and also at higher pressures in most of these arteries. However, NEpi never produced constriction in arterias subjected to procedures designed to kill living tissue, 
indicating that the constricting action of $N E p 1$ is dependent upon a living process. Presumably this process is the active contraction of smooth muscle cells, although the present experiments do not provide any direct evidence for this. The doses of NEpi used in the present experiments were quite high, although not as high as the doses used in some experiments reported in the literature; for example, Van Citters et al (1962) used 100 $\mathrm{g} / \mathrm{ml}$ dose for direct infiltration into mesenteric arteries. Others, such as Speden (1960) and Keatinge (1966) used doses of 500 and $250 \mathrm{~g} / 100 \mathrm{ml} \mathrm{NEpi} \mathrm{respectively,} \mathrm{similar} \mathrm{to} \mathrm{the} \mathrm{doses}$ used in the present experiments. Apparently the route of administration is an important variable. Gillespie (1966) studied the response of isolated, in vitro arteries to doses of NEpi perfused through the lumen as compared with the same concentration applied to the outside of the vessel. The arteries were found to be less sensitive to external applications of the drug. This is probably due to the bulk flow provided by the vasavasorum which transports the lumenal contents into and through the wall. In order for an externally applied drug to be effective it must diffuse through the adventitia and into the media. At 200-300 $\mathrm{mm} \mathrm{Hg}$, the pressure at which NEpi was given in the present experiments, the arterial wall was found to be about 0.25 $\mathrm{mm}$ thick. The smooth muscle is located in the middle third of 
the wall so that the diffusion distance was 0.10-0.15 mm. Since the drug was applied in high concentrations on or near the adventitia, it seems reasonable to assume that an appreciable amount would reach the smooth muscle cells: The purpose in using 200, 9/100 $\mathrm{ml} \mathrm{NEpi} \mathrm{was} \mathrm{to} \mathrm{produce} \mathrm{strong} \mathrm{contractions,} \mathrm{but}$ no claim is made that the observed response is the most powerful response possible.

Potassium cyanide was used to inhibit smooth muscle contraction. Diffusion considerations regarding NEpi apply equally to KCN. The observation that NEpi never elicited a contractile response after poisoning with cyanide suggests that smooth muscle contractility was very strongly inhibited. The short latency of the dilation response ioliouling adninistration of KCN suggests that the high $\mathrm{pH}$ may have been the primary cause of smooth muscle inhibition, rather than the inhibition of cyanide ion on metabolism. The effects of an alkaline pH on conrective tissue elements were of serious concern. However, the fact that arteries poisoned by diverse means (Table I) failed to exhibit any change in caliber when subsequently treated with 60 $\mathrm{mg} / 100 \mathrm{ml} \mathrm{KCN}$ allayed these fears. In 2 experiments, contracted vessels were treated with $68 \mathrm{mg} / 100 \mathrm{ml} \mathrm{KCl}$ to provide the same concentrations of potassium ion as that given when the vessels were treated with $60 \mathrm{mg} / 100 \mathrm{ml} \mathrm{KCN}$; this did not cause dilation, and in fact appeared to produce an additional constriction. The arteries were subsequently treated with $52 \mathrm{mg} / 100 \mathrm{ml} \mathrm{NaCN}$ to 
provide the same concentrations of cyanide as that given when the vessels were treated with $60 \mathrm{mg} / 100 \mathrm{ml} \mathrm{KCN}$; and this did cause dilation. The KCI did not alter $\mathrm{pH}$, while the NaCN elevated it to 9.0 . It is clear from this evidence that the inhibition of smooth muscle activity by $K C N$ is due to the action of the $\mathrm{pH}$ and/or the cyanide ion.

The present results (Figure 15) indicate that activation of the smooth muscle with NEpi does not change the compressibility characteristics of the arterial wall.' However, the slope of the wall volume curve after poisoning with cyanide might indicate a greater tendency toward compression. It seems likely that this is due to an expression of water. All the arteries in these studies were subjected only to ascending pressure sequences, and this could have resulted in a systematic decrease in volume as the pressure (and diameter) was increased. Unfortunately, neither random nor repeated sequences were performed. Casual observation of cyanide-treated vessels in the

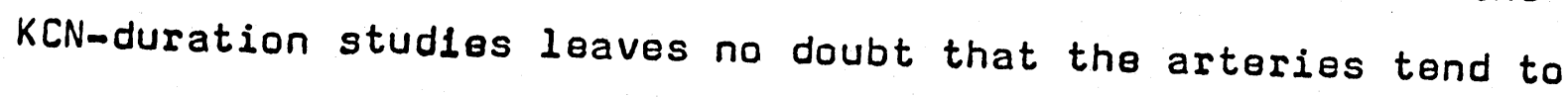
become water-logged. It seems probable that this tendency to become water-logged is due to the influence of the KCN upon various ion pumps and other systems limiting membrane permeability. The calculated wall volumes of vessels coated with lead after soaking in KCN for 60 minutes or more did not exhibit any tendency to decrease in volume with increases in radius. C. Specificity of NEpi and KCN. The arteries listed 
in Table I were subjected to several procedures to test the possibility that $N E p i$ and $K C N$ significantly, influerce the mechanical properties of arteries by an action other than excitation and inhibition of the vascular smooth muscle. It is evident that the first four procedures - freezing, heating, subjection to anoxia, and swelling with distilled water - inactivate the muscle because NEpi did not constrict these arteries, and KCN did not dilate them. However, it is possible that KCN dilates intact arteries by disrupting the integrity of some aspect of the connective tissue, and that the four procedures selected to inactivate the muscle have the same disruptive action on that aspect of the connective tissue. They would thus conceal the action of cyanide. However, it seems improbable that four such diverse methods of killing living tissue would share the same potential to damage elastin or collagen.

Freozing kills living cells if either the cooling or heating phases occur slowly. According to Prosser and Brown (1961) the mechanism of destruction results from: 1) disruption of the protoplasm by ice crystals; and 2) intracellular dehydration that results from the concentration of extracellular ions by the formation of $i c e$ crystals in the extracellular water before the intracellular water. However, the swelling produced by ice crystals might also break connective tissue elements. Heating inhibits the ATP-ase activity of muscle proteins. According to Fujino et al (1959) virtually complete 
inactivation of rabbit skeletal muscle actomyosin ATP-ase activity occurs after heating of $50^{\circ} \mathrm{C}$ for 15 minutes. Similarly, DeVillafranca and Naumann (1964) reported that the ATP-ase activity of actomyosin extracted from Limulus was almost entirely abolished by heating to $45^{\circ} \mathrm{C}$ for 10 minutes. The one hour heating procedure performed in the present experiments should have accomplished such ATP-ase inactivation. Care was taken not to exceed $50^{\circ} \mathrm{C}$ to avoid damaging collagen. Thermal contraction of native collagen occurs at $60-65^{\circ} \mathrm{C}$ (Gustavson, 1956; Piez and Gross, 1960), however more subtle denaturation might occur at lower temperatures. Elastin seems almost indestructable and can even withstand bolling in acid.

Soaking in distilled water was performed to cause swelling and lysis of cellular elements in the arterial wall. The obvious swelling with distilled water, and the abnormal ionic environment, itself, may have been detrimental to the muscle, enzyme systems and perhaps connective tissue. However, all preparations were restored to Krebs-Ringer solution before testing with NEpi and KCN. Perfusing arteries with nitrogen gas for 8 hours deprives cells of oxygen, and is probably the most innocuous of the 4 procedures. These various means of destroying cellular elements appear to be fairly independent of one another in their probable actions on the connective tissue. It seems reasonable to conclude, therefore, that the 
primary action of both NEpi and KCN is probably on the contractile activity of the vascular smooth muscle.

The fifth group of arteries listed in Table I were those treated with NEpi and KCN after storage under refrigeration in Krebs-Ringar dextrose solution for up to 12 hours. Three out of four of these arteries exhibited dilation in response to treatment with NEpi at $225 \mathrm{~mm} \mathrm{Hg}$. When the pressure was decreased to $150 \mathrm{~mm} H g$ they exhibited typical, slow, smooth muscle contraction. These were the only vessels ever observed to exhibit dilation in response to treatment with NEpi. Subsequent treatment with KCN produced dilation, and it was noted that the radius achieved at $225 \mathrm{~mm} H g$ by dilation with NEpi. NEpi and KCN were ineffective in eliciting responses from arteries killed by freezing, heating, etc., but were effective in eliciting responses from refrigerated arteries. This indicates that the smooth muscle in the latter group of arteries was probably responsive, and this interpretation is supported by the finding of Macht (1914) who reported that rings of human pulmonary artery, kept under refrigeration for 7 weoks, contracted in response to treatment with epinephrine. In the present experiments it was found that the vessels were very inextensible after standing in Krebs-Ringer solution without distention. The longer they were permitted to remain undistended the more inextensible they appeared to be. Thay also exhibited considerable 
creep at each pressure step. Zatzman et al (1954) have corIelated the viscous-like behavior of arteries with the amount of smooth muscle that they contain. Apter (1967) has made the same observation after studying the differential effects of temperature on the constituents of the arterial wall. In the present experiments it was also noted that the vessels exhibited much more creep after treatment with $N E p i$ than after poisoning with KCN. It therefore appears that long periods in the unloaded state permitted the arteries to contract to an extreme degres and to become very inextensible, as though an exceptionally large number of actin-myosin bonds had been formed at a shortened length. A few arteries were soaked in cyanide prior to mounting. These vessels did not exhibit the extreme inextensibility of live vessels. It appears, therefore, that a tonic contractile process is responsible for the resistance to distention, and, of course, that it is intracellularly located. In skeletal muscle, resting tension is usually

thought to be localized in extracellular structures, largely because of the dominance of the studies of Banus and Zetin (1938) and Ramsey and Street (1940). These authors demonstrated that microdissection of muscle fibers or compression of the fibers with fine dissecting needles to destroy intracellular contents had no effect on length-resting tension diagrams. They therefore attributed all of the resting tension to the 
sarcolemma. However, several less-quoted studies have refuted these findings. Casella (1950) reported that the resting tension of frog skeletal muscle fibers fell to $38 \%$ of control after compression of the muscle fibers with dissecting needles. Similarly, Buchthal and Weis-Fogh (1956) found that compression of the fibers of striated locust and water beetle muscles reduced their tensile strangth to $15-30 \%$ of control. Experiments described by Feng (1932), Sandow (1966), and Johnson and Garber (1967) may be interpreted as evidence for the view that the contractile system may bo involved in resting tension. Feng (1932) found that the oxygen consumption of resting skeletal muscle increased in proportion to the load that it was required to sustain. Sandow (1966) described the phenomenon of latency as a brief decrease in the resting tension of skeletal muscle which immediately precedes the rise in contractile tension. Finally, Johnson and Garber (1967) found that certain molluscan muscles are much less resistant to extension immediately after a phasic contraction than during normal resting conditions. These reports may be interpreted as evidence for the contribution of the contractile system to resting "tone". A similar interpretation may also be applied to the tonic behavior of the refrigerated arteries in the present experiments. Whether or not there is a significant "resting tension" within the vascular muscle in addition to the "tonic tension" of the contractile system cannot be determined from the present experiments. 
It appears that the refrigerated arteries are tonically inextensible and are capable of developing active tension when treated with NEpi. However, in order to contract they must first be released from their tonically contracted state. Treatment with KCN will release them, but then they will be unable to reform bonds. NEpi will also somehow release them and bonds' will reform, but not if the load opposing those bonds is too great. Apparently at $225 \mathrm{~mm} \mathrm{Hg}$ the distending force is too great for bonds to form, and at $150-175 \mathrm{~mm} \mathrm{Hg}$ it is not too great. An hypothesis, which may explain the NEpi-release behavior has been devised involving the interaction between the contractile element and a series elastic element. It will be discussed in a later section in which this model is developed. D. Shortening Bohavior. The present results indicate that the active contractile stress of vascular smooth muscle exhibits a unimodal peak when plotted as a function of strain. This agrees with the findings of many studies on smooth muscle preparations derived from arteries (Speden, 1960; Sparks and Bohr, 1961; Lundholm and Mohme-Lundholm, 19,66), intestine (Brocklehurst, 1926), taenia coli (Aberg and Axelsson, 1965), dog retractor penis (Winton, 1926), nictitating membrane (Hampel, 1934), uterus (Csapo and Coodall, 1954) and various molluscan smooth muscles (Abbott and Lowy, 1958a).

The mean strain at which the peak active stress 
occurred in the present experiments was $.56 \pm .04$, as compared with $60 \%$ to $90 \%$ extension reported by Speden (1960), Sparks and Bohr (1962) and Lundholm and Mohme-Lundholm (1966) for studies on strips. However, these strains are not truly comparable. "Initial length" in strip studies wero defined as the length associated with elther zero or some arbitrary level of tension, while in the present experiments "zero strain" was arbitrarily designated as the radius at $25 \mathrm{~mm} \mathrm{Hg}$ wile under the influence of NEpi. This value was selected because the $25 \mathrm{~mm} \mathrm{Hg}$ was used in most experiments as the low point in the pressure cycles. Another methodological difference between the present experiments and those found in the literature must be considered. The strip studies described in the literature were performed under essentially isometric conditions, while the present experiments were neither purely isometric nor purely isotonic. Thus, the calculation of active stress was based upon the capacity of the vessels to decrease dimension at each pressure. Since measurements were taken only after stable radii were achieved, the strain associated with the maximum active stress was inevitably smaller than would be measured under isometric conditions.

An inevitable error committed in performing the present experiments was in reestablishing the length of the arterial segments. When a retracted, thick-walled artery is drawn over a cannula and secured, a disproportionately large amount 
of wall material is trapped on the distal side of the ligature. When the free portion of the preparation is elongated, cannulated and secured, the central portion is inevitably over-extended. The only way to avoid this error is to cannulate the arteries in situ, and to fix their length before excision, but this was not performed in any of the present experiments. On the other hand, the studies on strips suffer from exactly the opposite error. These preparations are disproportionately thick at the onset and are unrestrained in all directions. Thus, while the present preparations probably tended to be overly inextensible, arterial strip preparations undoubtedly suffer from just the opposite property. .

The peak active stress observed in the present experiments was $.88 \pm .11 \times 10^{6}$ dynes $/ \mathrm{cm}^{2}$. This may be compared with only three studies in the literature, because in none of the others was the cross-sectional area determined. Abbott and Lowy (1953) reported that various moliuscan smooth muscles exhibited peak active stresses of 0.5 to $4.5 \times 10^{6}$ dynes $/ \mathrm{cm}^{2}$; Aberg and Axelsson (196.5) reported that guinea pig taenia coli exhibited a peak active stress of $1.8 \pm .5 \times 10^{6} \mathrm{dynes} / \mathrm{cm}^{2}$; and Lundholm and Mohme-Lundholm (1966) reported that strips of bovine mesenteric artery exhibited a peak active stress of 2000 grams $/ \mathrm{cm}^{2}$. However, these values are for whole tissue, and no attempt was made to express the data in terms of the percentage 
percentage of the tissue that is smooth muscle.

Goodford and Hermansen (1961) determined that the indulin space of guinea pig taenia coli is $320 \mathrm{ml} / \mathrm{L}$. If this value is taken as an iridication of the ratio of extracellular to intracellular space then this ratio may bo used to correct the active stress data of Aberg and Axelsson (1965). Thus corrected, the smooth muscle of taenia coli generates $2.7 \times 10^{6}$ dynes $/ \mathrm{cm}^{2}$ active stress.

Ducret (1930) determined, by histological studies, that smooth muscle comprises $75 \%$ of the cross-section of mesenteric arteries. If the active stress data of Lundholm and Mohme-Lundholm (1966) are corrected for this proportion a value of $2.67 \times 10^{6}$ dynes $/ \mathrm{cm}^{2}$ active stress is obtained.

Finally, Laszt (1964) determined, by histological studies, that smooth muscle comprises about one-third of the cross-section of bovine carotid artery. If the present experimental data are corrected for this proportion, a value of 2.64 $\times 10^{6}$ dynes $/ \mathrm{cm}^{2}$ is obtained. Thus, all three measurements performed on separate preparations, under different conditions and by different techniques are extremely close when corrected for the percent of the tissue that is actually muscle cells. The three studies do share one very important feature in common; each of them included a specific step to be certain that the muscle system was truly relaxed. Aberg and Axelsson used 
yoling and epinephrine to render the taenia coli segments atonic. Addition of monoiodoacetic acid failed to further elongate them. Lundholm and mohme-Lundholm used monoiodoacetic acid buffered to $\mathrm{pH} 7.9$ to kill the sirips of mesenteric artary. Potassium cyanide at $\mathrm{pH} 8.8$ was used in the present experiments. to kill the muscle. A special effort to inactivate the muscle is a vital step when studying smooth muscle because of the capacity of this tissue to maintain tone. The use of such a step may explain the closeness of the values that were obtained.

The present findings on smooth muscle also may be compared with skeletal and cardiac muscle. Studies on fibers obtained from frog semitendonosus muscle indicate peak active stresses of 2.4 to 4.4 (mean 3.5$) \times 10^{6}$ dynes $/ \mathrm{cm}^{2}$ (Ramsey and 9reet, 1940), while Spiro and Sonnenblick (1964) reported that frog sartorious muscle exhibits a peak active stress of 1.5 to 3.5 (mean 2.35) $\times 10^{6}$ dynes $/ \mathrm{cm}^{2}$. Studies on cat cardiac papillary muscle by the same authors indicate a peak active stress of $0.35-0.95$ (mean 0.64 ) $\times 10^{6}$ dynes $/ \mathrm{cm}^{2}$, while Walker (1960) reported values of $.23 \times 10^{6}$ dynes/ $\mathrm{cm}^{2}$ for dog papillary muscle. On the basis of these comparisons, it appears that smooth muscle can develop about the same active stress as skeletal muscle, and that both can develop 4 to 10 times that of cardiac papillary muscle. 
It is difficult to relate the presence of a peaked active smooth muscle stress curve to intracellular processes because so little is known about the disposition of smooth muscle proteins. The characteristics of the overlapping of actin and myosin have been correlated with the peaked length-tension curve in skeletal muscle (Huxley, 1960; Carlson et al, 1961) and cardiac muscle (Sonnenblick et al, 1964), but in smooth muscle the location of myosin is unknown. The presence of myosin has been established by chemical studies (Neodham and Williams, 1963; Schoenberg ot al, 1966), but not by alectronmicroscopic or $x$-ray diffraction studies (Rhodin, 1962; Elliott, 1964). This suggests that myosin exists in a disaggregated form. Panner and Honig (1967) described electronmicroscopic studies of smooth muscle which revealed parallel layers of actin filaments. They also observed arrow-head-Iike structures protruding lateraliy from actin filaments in extracted material. They therefore suggested that myosin might be located as dimers or other short units linking parallel actin filaments. Such a model with a random distribution of myosin units along the length of the actin filaments could account for the appearance of a peak in the active tension at moderate strains. The fairly symmetric decline of active tension above and below the peak are also consistent with this model. 
The present experimental data indicate that activation of smooth muscle with NEpi causes a gradual, graded contraction, bringing each artery to a smaller radiús after treatment with NEpi than during the pretreatment condition or during the period following poisoning with KCN. This decrease in radius is far more important to the overall status of the cardiovascular systom than the stress that can be developed. Patterns of blood flow are largely determined by the relative resistance in various vascular beds, and resistance is inversely related to the fourth power of the radius. It is therefore meaningful to consider the mechanics by which smooth muscle produces changes in radius.

According to Burton (1951), the connective tissue in the arterial wall provides "elastic tension" which can maintain equilibrium with the distending forces; smooth muscle is beliaved to provide additional "active tension" which permits the establishment of a new equilibrium at a lower radius, or at the same radius with a greater pressure. "Elastic tension" is believed to decrease with decreasing radius, reflecting the influence of collagen at large radii, and elastin at low radii. "Active tension" is believed to depend largely upon vasomotor activity. However, the problem persists as to how a graded contraction can occur. At equilibrium, the circumferential distending and restraining forces are equal. At constant pressure, 
the addition of even the slightest additional restraining force by smooth muscle should upset this equilibrium in favor of the restraining force and lead to a decrease in radius. Once this occurs the retractive force should always dominate because the distending force $\left(F_{d}=P_{T} \times r_{i} \times L\right)$ has been reduced by the decrease in radius. Since the $K C N$-poisoned vessel can maintain a stable equilibrium at all pressures it must be concluded that the elastic tension provided by connective tissue must decrease with decreasing radius, and that this then leads to a now equilibrium. However, even if the connective tissue "elastic tension" ( $\left.F_{R}={ }^{O_{C}} \times h \times L\right)$ were to decrease with decreasing radius, the additional force provided by the active muscle may always cause disequilibrium in favor of the restraining force. In addition, the wall thickness factor $(h)$ increases with decreasing radius, and this also tends to increase the restraining force. According to this line of reasoning, maximum constriction must result from even the slightest increase in smooth muscle activity. Obviously, this does not actually occur, not even in the case of the present experiments where very powerful smooth muscle contractions were elicited. The critical paramẹter is the circumferential stress $\left(o_{C}\right)$.

It may be observed in Figures $17 \mathrm{~A}, \mathrm{~B}$ and $18 \mathrm{~A}, \mathrm{~B}$ that the cyanide-poisoned vessel is capable of maintaining equilibrium over the entire pressure ranges studied. Thus, under 
relaxed conditions, any decrement in the distending force leads to a decrease in radius, and this leads to a decrement in the restraining stress. Activation of the muscle at large radii generates a relatively large increment in active stress, and this causes a decrease in radius ( $F$ igures $17 \mathrm{~A}$ and $18 \mathrm{~A}$ ). At large strains this decrease in radius causes a precipitous decline in the passive stress exerted by the wall (Figures 178 and 188). It is so great a decline in stress that even though the active stress is large, or even increasing with the decreasing radius, the net effect upon the total retractive stress (NEPi curve) is still an overall decline. Thus, the retractive stress within the wall declines as the radius decreases, and the radius will continue to decrease until the restraining force just equal the distending force. A new equilibrium is thus achieved, but because of the additional restraining force contributed by the muscle, the new equilibrium is achieved at a radius somewhat smaller than would occur if the muscle were relaxed. The only way to cause additional contraction is to produce a condition where the restraining force again exceeds the distending force. Decreasing the pressure will accomplish this by decreasing the distending force, and, again, the artery will begin to contract. At first, contraction will be rapid because there is a large force gradient. It then will proceed more and more slowly as 
the restraining stress declines with the decreasing radius, and the force gradient is gradually reduced with contraction. This self-limiting process thus achieves a new equilibrium with each pressure change.

The rate at which the passive stress ( $F$ igures 178 and 188, KCN curve) declines determines the magnitude of the radius change of the resting vessel, when subjected to equal step decreases in pressure. At large strains, a $25 \mathrm{~mm} H g$ decrease in pressure must produce only a small decrease in strain before a very large decline in passive stress occurs. At small strains, the same $25 \mathrm{~mm}$ Hg pressure step must produce a very large decrease in strain before an appreciable decrease in passive stress has occurred. Thus, the KCN pressure-radius curves ( $F$ igures $16 \mathrm{~A}$ and $17 \mathrm{~A}$ ) exhibit little radius change with given pressure decrements at large radii, but show very large radius changes with the same pressure decrement at smaller radii. The constricting influence of active smooth muscle depends upon how much additional restraining force it can provide. At large strains the active stress is great, and this is associated with large decreases in radius; at smaller strains the active stress is small and the magnitude of the decrease in radius is also small. However, the magnitude of active shortening is not simply a function of the absolute increment in force provided by the muscle, as one might predict; it is a 
function of how the force provided by the muscle modifies the slope of the stress-strain curve. Figures 178 and 188 indicate that at large strains the slope of the active-plu's-passive (NEpi) curve declines more gradually than the passive (KCN) curve at the same strain. At small strains the slope of the curve describing the KCN-treated vessel is steeper than that of the NEpi-treated vessel, and at moderate strains their slopes are about equal. This would predict that at large strains the NEpi-treated vessel will retract more, with a given pressure decrement, than the KCN-treated vessel. At low strains the KCNtreated should retract more than the NEpi-treated vessel. At moderate strains their retractions should be about equal. Inspection of Figures $17 \mathrm{~A}$ and $18 \mathrm{~A}$ indicates that these shortening patterns actually do occur.

It is known that decreasing the driving pressure in a perfused physiological preparation will cause a decrease in flow, and that the flow will cease before the gradient has fallen to zero. This phenomenon has been called "critical closure". Burton (1951) observed that critical closure is most apparent in arterioles and arterio-venous anastamoses, and that these vessels contain very little connective tissue. He argued that the caliber of these arteries was inherently unstable without connective tissue in their walls to maintain resting equilibrium; if the muscle were relaxed the distending force 
Mould overwhelm the negligible restraining force and cause extreme dilation, and if the muscle were active the distending force would be overwhelmed by the restraining force and cause complete closure. While the analysis of maximum dilation upon muscle relaxation seems reasonable, it is not immediately apparent why complete closure should not also occur in arteries in which there is an abundance of elastin and collagen. If the connective tissue and the smooth muscle behave as parallel, extended, elastic elements, such as stretched springs, then both of these elements may be expected to exert retractive forces. This premise underlies the subtraction of the stress after cyanide from the stress after NEpi as a means of isolating the stress exerted by active muscle. If this assumption of paratNel, retractive function is true, then one might anticipate a greater tendency for closure in arteries containing large amounts of connective tissue than in vessels containing little connective tissue. Burton's argument that there is "inadequate elastic tension to maintain equilibrium" and prevent closure is obscure.

An alternative interpretation originates with the observation in the present experiments that at zero $\mathrm{mm} H \mathrm{Hg}$ transmural pressure the arteries were always more dilated after poisoning with cyanide than after stimulation with NEpi. Inimost 
cases they were about the same radius at zero $\mathrm{mm} H g$ after cyanide as they were at $25-35 \mathrm{~mm} \mathrm{Hg}$ after treatment with NEpi. The cyanide poisoned vessel at zero $\mathrm{mm} H \mathrm{Hg}$ is probably the best approximation of the unloaded, natural state, because the forces within the wall are due entirely to the interaction be-. tween connective tissue components within the wall, and the elongated, cylindrical geometry. Under these conditions the circumferential stress is effectively zero. All the elastin and collagen fibers which tend to retract further are just counterbalanced by those which tend to be compressed. This may be termed the "zero state", and is probably the most logical designation of a true zero strain for the in situ length artery. A force, extrinsic to the connective tissue, is required to reduce the radius to below this level, and active smooth muscle can provide this force. Thus, at large radii the retractive force provided by smooth muscle adds to the force provided by the connective tissue fibers because they are both tensile, i.e. they both behave as extended springs. At radii below the zero state, the retractive force provided by the smooth muscle is opposed by the connective tissue fibers because the latter are subjected to a net compression. According to this interpretation, the reason why critical closure occurs in arterioles and arterio-venous anastamoses is simply that there is very little connective tissue present to resist compression. 
E. Distention Behavior. The data illustrated in. Figures $17 A, D, E, F$, and $18 D, E, F$, indicate that the elasticity of arteries tends to increase with both strain and pressure. The results of these sample experiments are substantiated by the data presented in Tables IIIA and IIIB. The general alinear form of the stress-strain curves (Figures 170 and 180) resembles those described in the literature by Roy (1880), Reuterwall (1921), Bramwell ot al (1923), Krafka (1939(, Roach and Burton (1959), and others who studied rings, strips, and pressure-volume segments. The biphasic character of this curve has most satisfactorily been explained by a parallel arrangement of elastin and collagen (Reuterwall, 1921; Remington et al, 1945; Roach and Burton, 1957). The low elasticity observed at small strains (Figures 170 and 180 ) has been attributed to elastin, while the increased elasticity encountered at longer strains has been attributed to collagen. Differential removal of either element by putrifaction (Remington et al, 1945; Krafka, 1939) or enzymic treatment (Roach and Burton, 1959) causes the wall to assume the behavior of the remaining element. The fact that the arterial wall does not disintegrate after differential removal of elastin or collagen proves that these elements are essentially arranged in parallel. If they were in series, removal of either element would destroy the wall. Apter (1967) differentiated elastin and collagen by their 
characteristic mechanical responses to elevated temperatures. These indicated that a collagen component is present even at small strains. However, this component is quite small, so that the overall static bohavior of relaxed or poisoned arteries may be described by the model illustrated in Figure 28. The "loose spring on the left, labelled E, represents elastin (elastic modulus about $3 \times 10^{6}$ dynes $/ \mathrm{cm}^{2}$ ), and the "tight" spring on the right, labelled C, represents relatively stiffer collagen (elastic modulus about $1-10 \times 10^{8}$ dynes $/ \mathrm{cm}^{2}$ ). The tight spring is connected to the loose spring by means of a detachable hook, in order to account for the fact that the high elasticity of collagen does not dominate the system until the system is extended to fairly large strains. This proposed model agrees with both the mechanical behavior of arterial preparations in the present and. many published studies, and with the electronmicroscopic finding of Pease and Paule (1960) who found that collagen fibers are not connected to any other structures within the wall. Burton (1954) emphasized that adventitial collagen serves as a protective jacket at large distentions. However, histological studies of arteries fixed at various perfusion pressures (Wolinsky and Glagov, 1964) indicate that although adventitial collagen fibers are still somewhat slack at pressures up to $200 \mathrm{~mm} \mathrm{Hg}$, the medial collagen fibers are quite taut at these pressures. Therefore, an additional longer, tight spring might be included in 


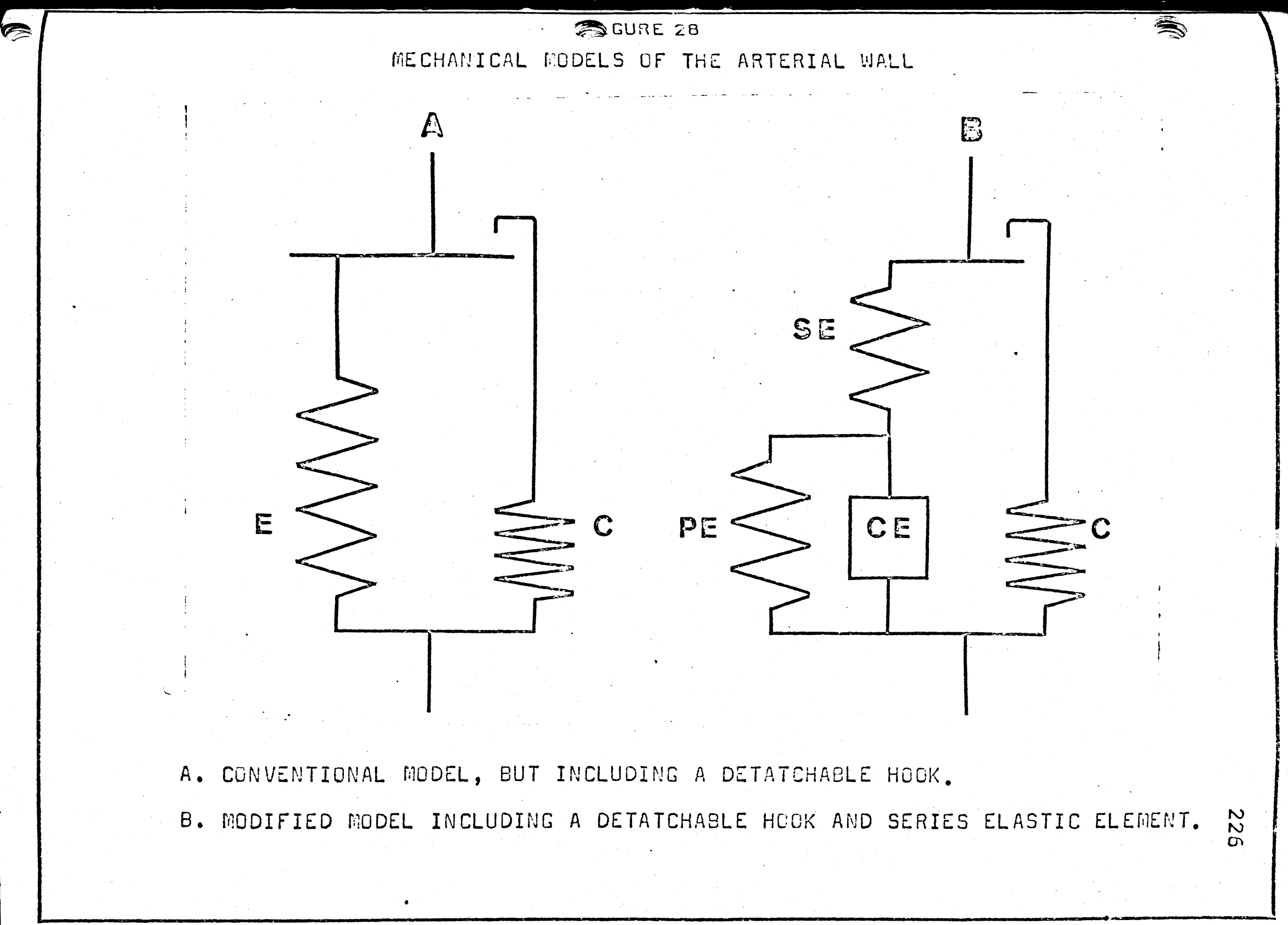


the model to represent adventitial collagen. It could be arranged so that its hook would not be engaged until the system was stretched to very large strains. The model predicts a sharp increase in elasticity when the $C$ tight spring is engaged, but the real artery is presumed to be composed of numerous similar model units. Variability in the lengtins of the springs would give a graded overall transition from the highly extensible to the very inextensible state.

The elasticity data observed in the presont experiments may be compared quantitatively with only those of Eergel (1961), because his are the only experiments performed on arterial segments held at the appropriate length and evaluated in terms of elastic moduli. The pressure-elasticity data presented in Figure 19 indicate that, at all but the lowest pressures, the values of Bergel fall between the values observed after treatment with NEpi and after poisoning with $K C N$ in the present experiments. This suggests that some residual activity of smooth muscle may have influenced Bergel's moduli, especially at high pressures. The difference between Bergel's and the present values in the $25-50 \mathrm{~mm} \mathrm{Hg}$ range is difficult to explain. One possibility is. that either his vessels were longitudinally slack, or that those used in the present experiments were somewhat overextended. This possibility is supported by a compariso 
between the present KCN data with Bergel's data obtainod from presumably relaxed vessels. These curves are shiftgd vertically apart over most of the pressuro range in a regular, systematic fashion. Another methodological difference betueen the present studies and those of Bergel is that his experiments were performed at $18-22^{\circ} \mathrm{C}$, in air, while the present experiments were performed at $36-37^{\circ} \mathrm{C}$, in a Krebs-Ringer bath. In order to evalvate the influence of temperature, 2 additional arteries were subjected to repeated pressure-radius cycles in the bath at $20^{\circ} \mathrm{C}$. After repeatable cycles uere obtained the temperature was elevated to $37^{\circ} \mathrm{C}$. Even though the final tuso cycles at $20^{\circ} \mathrm{C}$ were virtually identical, the next cycle, run at $37^{\circ} \mathrm{C}$, exhibited distinctly greater distensibility, especially at higher pressures. This suggests that Bergel's elasticity values may be somewhat higher than might be anticipated under in situ conditions.

The present data indicate that activation of smooth muscle with NEpi produces as increase in elasticity when this parameter is plotted as a function of strain, but a decrease in elasticity when it is plotted as a function of pressure. The consistency of this phenomenon is indicated by the daca in Table III. It has been previously noted that the elasticity of arteries increases with incroasing strain. Treatment with NEpi causes the smooth muscle to gonerate increased retractive force, 
and this causes contraction. In the present experiments the arteries were permitted to contract, and the elasticity was determined from the distention curves. Since the active smooth muscle caused circumferential shortening, the vessels exhibited smaller radii at each pressure level during the distention sequence after treatment with NEpi than during the distention sequence after poisoning with KCN. The elasticity of the retracted NEpi-treated artery is dominated by the mechanical characteristics of smooth muscle and relatively unextended elastin. By contrast, the elasticity of the dilated, KCN-poisoned artery is dominated by the mechanical characteristics of elongated elastin, and ultimately the extreme inextensibility of collagen. The present data indicate that the increase in elasticity provided by active muscle is insufficient to offset the decrease in elasticity that results from the contraction to a smaller radius. This point is illustrated by the location of the arrows in Figures 170 and 180 which indicate the strains associated with $100 \mathrm{~mm} \mathrm{Hg}$ in the NEpi-treated and KCN-poisoned statos.

A controversy has existed for some time concerning the action of smooth muscle on arterial distensibility. Wezler and Boger (1936), Wiggers and Uegria (1938), Landgren (1952), Alexander (1954), and Laszt (1968) found that excitation of vascular smooth muscle by administration of pressor agents or 
by means of reflex activation causes an increase in arterial distensibility. On the other hand, Lawton (1957), Leonard and Sarnoff (1957), Peterson et al (1960), and Torrance and Shwatz (1961) reported that excitation of vascular smooth muscle causes a decrease in arterial distensibility. If these data are considered in light of the present findings it may be observed that those reporting increased distensibldity presented their data as a function of pressure or stress, while those reporting decreased distensibility presented their data as a function of radius. Thus, the conflict seems more apparent than real. However, it is important to inquire as to whether or not conduit arteries actually do contract in vivo when the smooth muscle is excited. Local stimulation of arteries by electrical excitation or topical application of pressor agents causes constriction (Peterson et al, 1960; Davis, 1964; Keatingo, 1966) but these are not normal circumstances. Gerova and Gero (1967) directly measured tha in vivo diameter of femoral arteries under the influence of integrated, reflex-induced changes in sympathetic tone. Arterial diameter was measured with a displacement transducer similar to the device used in the present experiments. ' Sympathetic activation, produced by bilateral carotid occlusion, was associated with increased systemic blood pressure and increased arterial diameter, while inhibition of sympathetic tone by inflation of the carotid sinuses was associated with a decrease in systemic blood pressure and 
a decrease in arterial diameter. When the blood pressure was artificially stabilized by a system of reservoirs, reflex-induced sympathetic activation caused femoral constriction, and reflex inhibition of sympathetic activity resulted in femoral dilation. Thus, in the animal without pressure stabilization, the arterial diameter followed the changes in pressure. This indicates that true constriction probably does not take place in vivo. Patel et al (1960) measured the radius of dog pulmonary arteries in situ. They found that systemic administration of NEpi produced a small decrease in arterial distensibility, i.e., a decrease in the dimensional changes associated with the pressure pulse. However, they also noted an increase in the diastolic diameter, so that the decrease in distensibility might have resulted from increased elasticity associated with the increase in diastolic diameter. These results strongy suggest that true constriction of large arteries probably does not occur in vivo, and any effects that the activated smooth muscle might have would have to be exerted without the influence of gross shortening.

In order to investigate the influence of shortening, 7 arteries were subjected to pressure cycles which were designed to maintain the vessels within a narrow range of radii. When NEpi and, later, KCN were administered, the pressures were manipulated to keep the vessels at the low point in the 
narrow radius range so that no apparent shortening or dilation could occur. In 6 out of 7 experiments treatment with NEpi resulted in no change in distensibility at the lower'part of the radius range, but a definite increase in distensibility over the KCN-poisoned vessel in the higher regions of the radius range. One such experiment is illustrated in Figure 29. After poisoning with KCN the vessel became progressively stiffor with increasing radii, but after treatment with NEpi it continued to remain distensible, at these same radii. A striking feature noted was that treatment with NEpi caused the dia tensibility curve $(\Delta \mathrm{r} / \Delta \mathrm{p})$ to become absolutely linear over the $100 \mathrm{~mm} \mathrm{Hg}$ range tested. It is difficult to conceive of how smooth muscle could "add" distensibility to the fully relaxed (KCN) artery. Active contractile bonds must have formed because it was necessary to elevate the transmural pressure 35 to $50 \mathrm{~mm} \mathrm{Hg}$ to prevent more than a just-discernible decrease in radius. A possible explanation of the NEpi-induced increase in distensibility, based upon an assumption of isometric contraction of smooth muscle, is offered in a later section of this discussion.

F. Hysteresis. The present results indicate that arteries subjected to distention and retraction exhibit hysteresis. In all cases the distention process was associated with greater pressure and stresses than the retraction process. 
These results are similar to those reported for circumferencetension curves of aortic rings (Remington, 1955), pressurevolume curves of arterial segments (Landgren, 1952; Bergel, 1961), pressure-volume curves of venous segments (Alexander et al, 1953), pressure-volume curves of the total pulmonary vasculature (Sarnoff and Berglund, 1952), pressure-diameter curves of in vivo arteries (Remington, 1962), and length-tension curves of arterial strips (Speden, 196l; Sparks and Bohr, 1962; Lundholm and Mohme-Lundholm, 1966). In the present experiments it was found that the width of the hysteresis loop depends upon the amplitude of extension, $i . \theta$. the extent to which the radius was changed. Remington (1955) made the same observation regarding circumference-tension curves for arterial Rings. It was also observed in the present experiments that the vessels were much less extensible during the initial distention than upon subsequent distentions. This has been observed by Krafka (1939), Remington et al (1945), Katz et al (1947), Bergel (1961), and many others.

The present data indicate that activation of the smooth muscle with NEpi markedly increases the width of the hysteresis loop. This differs with the results of Remington (1955) who found that treatment of rings of femoral artery with epinephrine caused increased tension, but only on the Pirst extension following treatment. On the subsequent 
retraction and all following extensions the loop returned to its usual dimensions. However, the findings of Goto and Kimoto (1966) were similar to the present observations. Electrical stimulation of aortic rings increased the amplitude of hysteresis, and especially displaced the limb of increasing stress. Remington also observed hysteresis in ligamentum muchae and artery preparationsputrified long enough to destroy the smooth muscle cells, but not long enough to destroy the elastin. However, he did not observe hysteresis in collagenous tendon. Goto and Kimoto did observe hysteresis in arterial preparations treated with trypsin to remove the elastin, but did not see it if the collagen was removed with formic acid. Hirsch (1944) observed delayed recovery of strain in collagenous cartilage. In the present experiments on shortening and distention from multiple origins it was noted that the width of the loop depends upon the amplitude of extension, especially at pressures of $100 \mathrm{~mm}$ Hg or more. This agrees with the findings of Goto and Kimoto that chemical destruction of collagen abolishes the hysteresis of arteries; apparently collagen fibers begin to carry the load at these pressures. Remington's (1955) finding that collagenous tendon does not exhibit hysteresis does not negate the importance of arterial collagen with respect to hysteresis. Tendon is composed of closely packed, highly oriented fibers, while arterial collagen fibers tend to assume an increasingly random organization with 
Rdecreasing pressures.

Remington (1963) suggested that there might be structural changes as intramural elements shift from a circumferential to a longitudinal orientation. This may be important in untethered arterial rings, but it is probably not a dominant factor in the present fixed-length arteries. The most obvious explanation is that hysteresis is due to internal viscosity which prevents the dimensional changes from faithfully following the changes in force. Although viscosity undoubtedly contributes to hysteresis it does not seem to be the essential cause for the following reasons. It has been noted in the present experiment and many previous studies that the retraction limb of the loop is relatively stable, and that it is alterations in the extension limb which increase or decrease the width of the hysteresis loop. If hysteresis were caused by a viscous phenomenon then one would expect both limbs of the loop to be equally altered by an effective influence. Further more, Remington (1955) found that the width of the loop was not influenced by the rate of stretch until some critical value was encountered. Certainly any viscous phenomenon should be influenced by the rate of stretch. In the present experiments, 2 minutes of stable radius was used as a criterion of stability. The time constant of any visco-elastic element influencing hysteresis under these conditions must be so long 
that it seems justifiable to consider this influence essentially elastic. A model which may explain this stable hysteresis is presented in the following discussion.

Most descriptions of the arterial wall place smooth muscle in parallel with the connective tissue elements (Reuterwall, 1921; McDonald, 1960). However, this arrangement cannot account for the rather complex behavior observed in the present experiments. Lundholm and Mohme-Lundholm (1966) and Aberg (1967) reported the presence of a significant series elastic element in smooth muscle. Accordingly, a more satisfactory model has been devised which incorporates a series elastic element. This is illustrated in Figure 28B. The contractile element (CE) is coupled to both a series elastic felement (SE), and a parallel elastic element (PE). Collagen (C) is arranged parallel to this system, connected by means of a detachable hook. Certain assumptions are made about the behavior of the various components of the model at and below physiological strains: 1) the force increment necessary to extend the CE a given strain is greater than the force decrement necessary to shorten the $C E$ an equivalent amount, i.e. its elastic modulus at any given strain is greater when undergoing extension than when undergoing retraction; 2) the CE exhibits a length-elasticity relationship, with the maximum elasticity occurring at approximately the same strain as that 
correlated with the maximum active stress; 3) the elasticity of the $C E+P E$ is greater than that of the $S E$, and it changes more with a given change in strain than does the elasticity of the SE. The rationale for these assumptions is as follows:

1) the force increment required to extend to $C E$ seems to be greater than the force decrement required to shorten the vessel because the retracted, NEpi-treated artery exhibits less distensibility $(\Delta \mathrm{r} / \Delta \mathrm{p})$ at most radii during extension than during shortening. This is not observed when the CE is inactivated by $K C N ; 2)$ the $C E$ is thought to exhibit a length-elasticity relationstip because it is assumed that the same intracellular contractile bonds responsible for shortening are responsible for resistance to extension. The shortening data reveal the Rpresence of a length-active stress relationship, so it is reasonable to assume that the resistance to extension exhibited by the contractile element may also follow such a pattern; 3) the elasticity of the CE is believed to be greater than the elasticity of the $S E$ and $P E$ because of the strain-elasticity data in Figures 170 and 180. The elasticity of the CE is believed to charige more than the elasticity of the SE and PE with equivalent changes in strain because at low and moderate strains the slopes of the NEpi elasticity-strain curves are steeper than the slopes of the KCN curves at low and moderate strains (Figures 170 and 180 ). This model, with its assumptions 
may be applied to the hysteretic behavior of the artery illustrated in Figure 20. Interruption of the distention limb of the hysteresis loop after NEpi produced different results when performed at large and small radii. Interruption of the distention limb at a low radius resulted in marked shortening with the very first pressure decrease. However, when the distention limb was interrupted at a large radius it did not shorten until the pressure had been decreased several steps. This behavior was not seen after the vessel had been poisoned with KCN. The model explains this behavior as follows. During the distention process after treatment with NEpi, both the SE and the CE are extended. Interruption of the extension curve at a low pressure or radius results essentially in a reverse process, and therefore little hysteresis. However, distention to a large radius results in a relatively greater extension of the SE than the CE because the CE becomes more elastic with in creasing length. The SE is assumed to be somewhat less elastic and it therefore continues to extend. Extension of the SE permits the overall radius of the artery to increase and this increases the distending force by more than just the pressure increase. This is because the distending force is equal to the produce of the pressure times the vessel radius. Interruption of the extension limb at a large radius does not result in active contraction. This is because the CE is too 
short, with regard to its position along its strain-active stress curve, to generate sufficient contractile force to overcome the high distending force associated with the large pressure and radius. Decreasing the pressure thus results, initially, in only passive retraction of the SE. Support for this interpretation is the similarity between the initial portion of the retraction curve of the vessel after treatment with NEpi and the retraction curve of the artery after poisoning with cyanide at approximately the same pressure. The vessel thus behaves as though only connective tissue elements (the SE) were retracting, but that these were "set" at an abnormally short circumferential length by the relatively less-extended CE. The fact that the NEpi curves tend to resemble the KCN curves at a slightly lower pressure may reflect the partially retracted PE. As the radius decreases along this curve the distending force falls until it is equal to the active force that the "set" CE can generate, and active contraction can then proceed.

Shortening from multiple origins was performed in order to obtain a comprehensive family of curves similar to those observed in the interrupted loop experiments. The model predicts that the disparity between the active stress that the smooth muscle can develop and the distending stress that it must overcome increases as the point of origin is increased. 
hat a large radius of origin the CE should be far too short to cause active contraction, while at a small radius of origin. it should be able to cause considerable contractions. Figures $21 \mathrm{~A}$ and $22 \mathrm{~A}$ indicate that this behavior is actually observed. When these data are converted to stress-strain curves (Figures 218 and 228), the difference between the KCN curve and any NEpi curve may be taken as an indication of the active stress. It is clear that the smaller the redius of origin, the greater the effective active stress that is developed at any given strain during the subsequent retraction sequence. This agrees with the prediction of the model that the disparity between the active contractile force and the distending force is increased with increased extension. Thus, in spite of the fact that r. b the CE is more extended at a large radius of origin, and potentially able to generate considerable force, it is unable to overcome the disproportionately larger distending force. Since the extended muscle cannot produce a decrease in vessel radius, the calculated stress associated with a given strain is lower for the vessel when originating from a large radius of origin then for when originating from a lower radius of origin. Subtraction of the KCN stress curve from the NEpi stress curves in figures 218 and 228 would give a family of active stress curves. Although this subtraction has not been performed, one may anticipate that these curves will indicate 
Nithe progressive extension of the $C E$ and $P E$ portion of the model, so that it should be possible to calculate the elastic modulus of just these parallel-coupled elements.

Distention from multiple origins (Figures $23 \mathrm{~A}$ and 24A) was performed in order to study the influence of successively shorter radii on subsequent distention curves. Application of the model to this procedure predicts that the CE will be permitted to retract to progressively shorter lengths the further the distending force is decreased. Thus, the curves originating from small radii of origin should possess a shorter CE, and therefore should exhibit lower elastic moduli than those originating from larger radii of origin. The graded elastic moduli presented in Figures $23 \mathrm{C}$ and $24 \mathrm{C}$ support this Pprediction. Thus, the ability of the "active" artery to retract or distend depends, to a good extent, upon its recent length and pressure history. According to the proposed model, the properties exhibited by an artery, at any given radius, re flects the influence of a wide range of combinations of $C E$ length and SE length.

The model may also explain the behavior of arteries maintained within a restricted range of radii (Figure 29). These experiments indicate that NEpi increases the distensibility over that observed in both the protreatment and $\mathrm{KCN}-$ poisoned vessel. The increase in distensibility was found 
Nith respect to both pressure and radius. No matter how small an elasticity the CE contributes to the system it must increase the elasticity over the $K C N-p o i s o n e d ~ v e s s e l$, unless it somehow takes away more elasticity than it adds. The model suggests that an isometric contraction might occur, so that the CE and PE would shorten and SE would extend. The degree to which the elastic moduli of the CE and PE are decreased by shortening must be quantitatively greater than the degree to which the elastic modulus of the SE is increased in order for a net increase in distensibility to occur. The one restricted radius experiment exhibiting a slight decrease in distensibility after treatment with $N E p i$ was performed at high radii and pressures, suggesting that the SE may have been in (6. the steep portion of its strain-elasticity curve.

The behavior of refrigerated arteries presents one of the most difficult challenges for the present model. The marked inextensibility coupled with NEpi-induced dilation after refrigeration suggests that the CE was providing the inextensibility. Consideration of the dilation response in terms of therimodel suggests that the large radius achieved at high pressures might have been due largely to an extreme extension of the SE element. Two observations are consistent with this viow: 1) the arteries dilated with NEpi to almost the identical radius achieved, at the same pressure, after poisoning with cyanide; 2) active contraction did occur when the 
pressure, and therefore distending force, was decreased. It may be that NEpi increases both the rates of "make" and "break" of the contractile bonds, or at least the rate of "break", if there is some inherent tendency to "make", NEpi would then release the locked $C E$, and permit almost maximum distention. However, it remains a mystery how so short a CE could exhibit such high elasticity. Several authors (Bader, 1963; Alexander and Johnson, 1965) have suggested the presence of a plastic element in series or parallel with the CE. Abbott and Lowy (1958) observed that when molluscan smooth muscle was permitted to remain under constant load overnight, it exhibited an almost identical length tension curve on the following day as it did on the preceding day, but did so at a longer length. A plastic element has not been incorporated into the present model because neither its location nor its properties can be consistently predicted.

G. Longitudinal Properties. The present studies indicate that the arterial wall bohaves as an anisotropic tube when elongated to in situ length, and that its anisotropy is. exaggerated by activation of the vascular smooth muscle. This is exemplified by the prompt decline in longitudinal retractive force that follows treatment with $N E p i$, even without any significant changes in radius. This might be attributed to an 
isometric contraction of the vascular smooth muscle. Such a contraction may have realigned connective tissue elements from a random or longitudinal orientation to a more circumferential orientation. Alternatively, the decrease in longitudinal force may have resulted from a change in the geometry of the muscle cells. Electronmicrophotographs indicate that the smooth muscle cells of the arterial wall are densely-packed, with the wide portion of each cell fitting neatly into the potential space provided by the narrow portion of cells beside it (Pease and Paule, 1960; Conti et al, 1964). If these cells shorten they must become wider, and there is virtually no space to accomodate this widening, without producing longitudinal compression. Such a compression force would tend to cause longitudinal elongation. These speculations are based on the assumption that the cells, as a whole, are capable of shortening without producing an overt change in arterial radius. It is also possible that activation with NEpi might cause an orientation of intracellular contractile filaments to a predominantly circumferential direction, which coincides with the long axis of the cells. Unfortunately, too little electronmicroscopic data is available to evaluate this possibility. However, the concept of reorientation seems to be real. One of the 5 arteries studied was arbitrarily mounted at a length that was longer than in situ length. In this vessel each increase in 
prossure, in tho pretrealment condition, resulted in an increase in longitudinal retractive force. When the vessel was treated with NEpi this pattern actually reversed 'so that each increase in pressure then resulted in a decrease in longitudinal force, resembling the other 4 arteries. Finally, it was seen that when the vessel was poisoned with $K C N$, it reverted back to the unique pretreatment behavior.

The conclusion that the wall is anisotropic has also been expressed by Fenn (1957), who found that retracted arteries elongated when subjected to increasing transmural pressures. Inspection of equation 20 indicates that an isotropic tube should not change length because the Poisson's ratio of 0.5 halves the value of $\frac{\sigma_{C}}{E}$; the circumferential stress due to pressure isjust double the longitudinal stress due to pressure, so that $\frac{\sigma_{L}}{E}=\frac{\sigma_{C}}{E}$; thus, $\varepsilon_{L}$ equals zero. However, arteries do not normally exist in a retracted state, and the additional forces involved in maintaining tether upset the $2: 1$ ratio between circumferential and longitudinal stress. It is of interest, therefore, that the present experimental data indicates that the arterial wall is anisotropic, even under in situ length conditions. For the purposes of comparison, 3 additional arteries were cannulated at both ends. One cannula was mounted to the tissue bath, and the other cannula was sealed and permitted to float freely on the Krebs-Ringer 
Brolution in the tissue bath. The vessels were inflated with oxygen from their mounted end and were photographed with a Contaflex $35 \mathrm{~mm}$ camera equipped with portrait lenses and an electronic flash unit. Vessel dimensions were determined by measuring the images of the vessels on the color slides under the dissecting microscope fitted with Cook imago-splitting eyepiece. The vessels were subjected to shortening and distention procedures under pretreatment, NEpi and KCN conditions, and were $x$-rayed at the end of the experiments. Figure $30 \mathrm{~A}$ illustrates the stress-strain curves for the shortening sequences. Both longitudinal and circumferential expansion occurred. Longitudinal expansion apparently limited the strains that could be achieved in the circumferential direction. Treatfont with NEpi elicited a typical increase in circumferential stress, but produced no measureable active stress in the longitudinal direction. This pattern was observed in each of the 3 experiments, and is in agreement with Barbour (1912) who found much less of a response to epinephrine from arterial strips cut in a longitudinal direction than from those cut in a transverse direction. Figure $30 \mathrm{~B}$ presents the strain-elasticity curves for the distention sequences. Unlike the shortening isequences, NEpi did have a measurable effect on both longitudinal and circumforential elastic moduli when plotted as a 
function of their respective strains. The elastic moduli in these experiments were calculated simply as the slope of the stress-strain curves. These experiments agree wh th the fixedlength longitudinal studies, in that both preparations exhibited a tendency to lengthen with increasing.pressure, and both showed an increase in longitudinal stress under the influence of NEpi during the distention sequences.

The studies on freely distensible arteries illustrated in Figure 308 indicate that the longitudinal elastic modulus increases abruptly with increasing length. The in situ length artery is fixed at a length which probably corresponds to a large longitudinal strain in Figure 308. At very low pressures the circumferential strain may decline to low values, but the fixed longitudinal strain cannot. This may explain the finding that at low circumferential strains, the circumferential elastic modulus may decrease to a value that is equal to, or even lower than, the longitudinal elastic modulus. The anisotropy observed in both freely-distensible and in situ length arteries indicates that a more complex mathematical approach is necessary for an exact analysis of the arterial wall. It also indicates the inadequacy of arterial strip and ring preparations where a precise determination of mechanical properties is desired. 


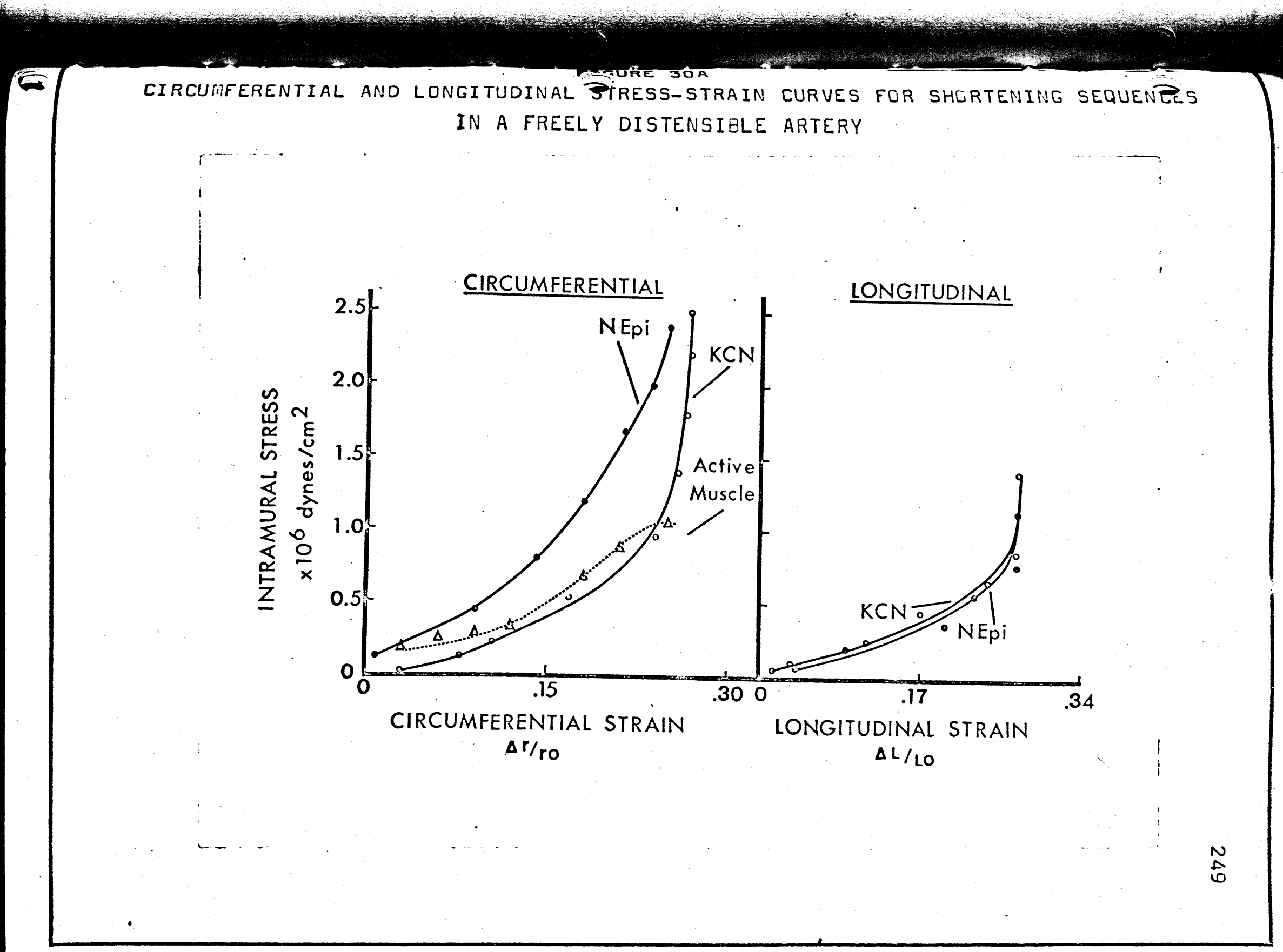




\section{CIRCUMFERENTIAL AND LONGITUDINAL ELASTIC MODULUS-STRAIN CURVES}

FOR DISTENTION SEQUENCES

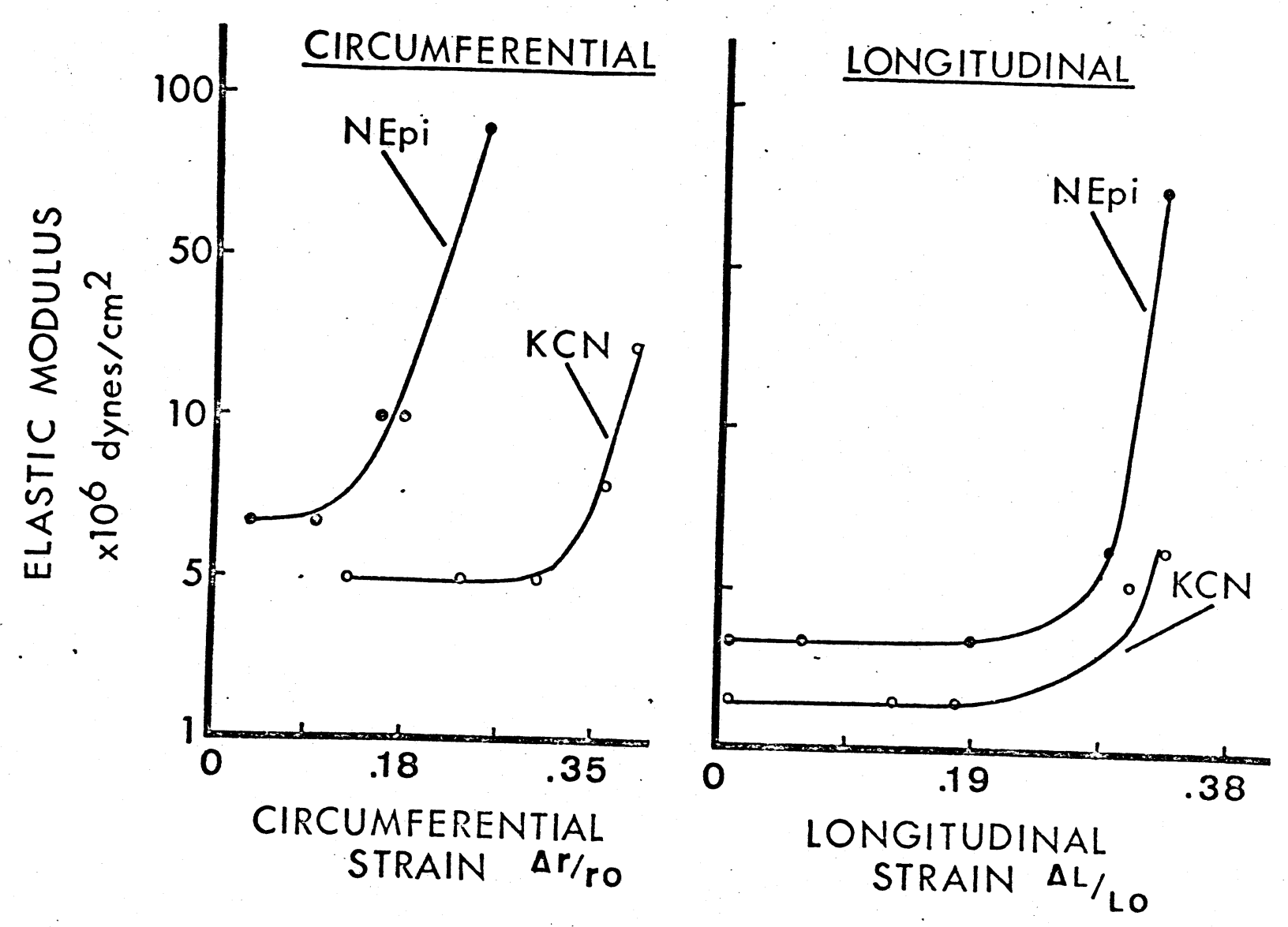


CHAPTER VI

\section{SUMMARY AND CONCLUSIONS}

Techniques were devised to measure the mechanical properties of in situ length carotid arteries under steadystate conditions. Radii were measured with a transducer consisting of a transformer with a light-weight moveable core; wall thicknesses were calculated from $x$-rays taken of the vessel at the end of the experiments. The following results were obtained: 1) the volume of the arterial wall remains constant over the normal physiological range of pressures, thus the Poisson's ratio is 0.5 . This was found when the smooth muscle was active (NEpi), moderately active (untreated), and killed $(K C N) ; 2)$ the activated smooth muscle caused active arterial constriction. The mean active circumferential stress developed by the smooth muscle of the carotid artery was .88 $\$ .11$ dynes $/ \mathrm{cm}^{2}$ for whole tissue, and $2.64 \times 10^{6}$ dynes $/ \mathrm{cm}^{2}$ for the estimated percentage of the wall that was purely smooth muscle; the maximum decrease in radius produced by activation of the muscle occurred at strains correlating with a mean pressure of $62 \pm 4.8 \mathrm{~mm} \mathrm{Hg} ; 3$ ) activation of the muscle produced a change 
(in circumferential elastic modulus of the vessels subjected to distention. The vessels exhibited a greater elastic modulus when the muscle was active than when it was poisoned, if considered at any given strain. Just the opposite was observed if the elasticity was considered at any given pressure; 4) hysteresis was observed, and it was greatly increased when the smooth muscle was activated. The hysteresis was such that the wall stress was greater at any given radius during distention than during shortening; 5) vessels permitted to shorten from various radii exhibited progressively less active stress at any given radius, the larger the radius from which the shortening procedure was begun. Similarly, vessels subjected to distention from various radii exhibited progressively lower 0 olastic moduli, the smaller the radius from which the distention was begun. These effects were greatest when the smooth muscle was active, and least when the smooth muscle was poisoned. A model incorporating a series elastic element was proposed to account for this behavior; 6) longitudinal forces were detarmined in some vessels, along with circumferential forces. Both in situ length and freely distensible arteries exhibited a tendency to lengthen when subjected to elevated transmural pressures. It was condluded that the arterial wall is anisotropic and exhibits a greater elastic modulus in the circumferential direction than in the longitudinal direction. 
1. Abbott, B.C. and J. Lowy. Mechanical properties of mytilus muscle. J. Physiol. 120: 50, 1953.

2. Abbott, B.C. and J. Lowy. Mechanical properties of helix and mytilus muscle. J. Physiol. 141: 398, 1958a.

3. Abbott, B.C. and J. Lowy. Contraction in molluscan smooth muscle. J. Physiol. 141: 385, 1958b.

4. Aberg, A.K.G. The series elasticity of active taenia coli in vitro. Acta Physiol. Scand. 69: 348, 1967.

5. Aberg, A.K.G. and J. Axelson. Some mechanical aspects of an intestinal smooth muscle. Acta Physiol. Scand. 64: 15, 1965 .

6. Abraham, A. Receptors in the walls of blood vessels. Hung. Acta Biol. 1: 157, 1949.

7. Alexander, R.S. Mechanics of vasoconstriction. Fed. Proc. 12: 3. 1953 .

8. Alexander, R.S. The participation of the venomotor system in pressor reflexes. Circ. Res. $\underline{2}: 405,19546$.

(1.9. Alexander, R.S. The influence of constrictor drugs on the distensibility of the splanchnic venous system, analyzed on the basis of an aortic model. Circ. Res. 2: $140,1954 \mathrm{a}$.

10. Alexander, R.S.., W.S. Edwards, and J.L. Ankeney. The distensibility of the portal vascular bed. Circ. Res. 1: 271,1953 .

11. Alexander, R.S. and P.D. Johnson. Muscle stretch and theories of contraction. Am. J. Physiol. 208: 412, 1965.

12. Appenzeller, 0. Electron microscopic study of the innervation of the auricular artery in the rat. J. Anat. 98: 87, 1964. 
13. Apter, J.T. Correlation of visco-elastic properties of large arteries uith microscopic structure. I. Methods used and their justification. Circ. Res. 19: 104, 1966.

14. Apter, J.T. Correlation of visco-elastic properties with microscopic structure of large arteries. IV. Thermal responses of collagen, elastin, smooth muscle, and intact arteries. Circ. Res. 21: 901, 1967.

15. Apter, J.T., M. Rabinowitz, and D.H. Cummings. Correlation of visco-elastic properties of large arteries with microscopic structure. II. Collagen, elastin and muscle determined chemically, histologically, and physiologically. Circ. Ros. 19: 111, 1966.

16. Armstrong, W.MCD. Sodium, potassium and chloride in frog stomach muscle. Am. J. Physiol. 206: 469, 1964.

17. Astbury, W.T. and F.O. Bell. X-ray data on the structure of natural fibers and other bodies of high molecular weight. Tabulae Biologicae 17: 90, 1939.

18. Astbury, W.T. The molecular structure of the fibers of the collagen group. J. Internat. Soc. Leath. Tr. Chem. 24: 69, 1940 .

Astbury, W.T., S.V. Perry, R. Reed, and L.C, Spark. An electronmicroscope and $x$-ray study of actin. Biochim. Biophys. Acta 1: $379,1947$.

20. Axelsson, J. and E. Bulbring. Metabolic factors affecting the electrical activity of intestinal smooth muscle. J. Physiol. 156: 344, 1961.

21. Ayer, J.P., G.M. Hass, and D.E. Philpott. Aortic elastic tissue: Isolation with use of formic acid and discussion of some of its properties. A.M.A. Arch. Path. 65: 519, 1958.

22. Ayer, J.P. and R. Eisenstein. Finer structure of aortic elastic tissue: A new strip technique. Laborat. Invest. 12: 864, 1963.

23. Bader, H. The anatomy and physiology of the vascular wall, Handbook of physiology, Section III, Vol. II, Chapter 26. Baltimore: Williams and Wilkins Co., 1963. 865 p. 
24. Bader, $H$. and E. Kapal. Altersveränderungen der Aortelas(i) tizität. Gerontologica 2: 253,. 1958 .

25. Bahr, G.F. Electron microscopic studies of collagenous and elastic tissue. Arch. Dermat. \& Syph. 193: 578,1951.

26. Bahr, G.F. Über die Feinstruktur elastischer Fasern. Z. Anat. 116: 134, 1951.

27. Balazs, F.A. and T.C. Laurent. Viscosity function of hyaluronic acid as a polyelectrolyte. J. Polymer Sci. 6: 665,1951 .

28. Balo, J. and I. Banga. Die Zerstorung der Elastischen

Fasern der Gefasswand Schweiz. Z. Path. Bakt. 12: 350, 1949.

29. Balo, J. and I. Banga. The elastolytic activity of pancreatic extracts. Biochem. J. 46: $384,1950$.

30. Banga, I. Structure and $\frac{\text { Function }}{\text { Budapest: Akademiai Kiado, }} \frac{\text { Elastin }}{1966 .} \frac{\text { and }}{291} \frac{\text { Collagen. }}{\mathrm{p} .}$

31. Banga, I. and J. Balo. Elasticity of the vascular wall. II. Effect of elastolytic enzymes on the elasticity of the human carotid in vitro. Acta Physiol. Acad. Sci. Hung. 20: 250,1961 .

(1)

32. Banga, I. and D. Schuler. Contributions to the structure of elastin with special reference to the action of elastase. Acta Physiol. Acad. Sci. Hung. 4: 13, 1953.

33. Banus, M.G. and A.M. Zetlin. The relation of isometric tension to length in skeletal muscle. J. Cell. Comp. Physiol. 12: 403,. 1938.

34. Barany, M., K. Barany, E. Gaetjens, and G. Bailin. Chicken gizzard myosin. Arch. Biochem. Biophys. 113: 205, 1966.

35. Barbour, H.G. Die Struktur verschiedener Abschnitte des Arteriensystems in Beziehung auf ihr Verhalten zum Adrenalin. Arch. f. Exp. Path. U. Pharm. 68: 41, 1912.

36. Barbour, H.G. The constricting influence of adrenaline upon the human coronary arteries. J. Exp. Med. 15: $404,1912$. 
37. Barcroft, J., J.F. Danielli, W.F. Harper, and P.D. Mitchell. Wharton's jelly considered as a conducting path. Nature 154: 667, 1944 .

38. Barr, L.M. Distribution of ions in intestinal/smooth muscle. Proc. Soc. Exp. Biol. \& Med. 101: 283, 1959.

39. Baumann, P. The present state of our knouledge in the field of elastomers. Chem. \& Indust. III: 1498, 1959.

40. Bear, R.S. Long $x$-ray spacings of collagen. J. Am. Chem. Soc. 64: 727,1942 .

41. Bennett, $M . R$. The effect of intracellular current pulses in smooth muscle cells of the guinea pig vas deferens. at rest and during transmission. J. Gen. Physiol. 50: 2459,1967 .

42. Benninghoff, A. Uber die Beziehungen zwischen elastischen Gerust und glatter Muskulature in der Arterienwand und ihre funktionelle Bedeutung. Ztschr. Zellforsch \& Mikroskop. Anat. 6: $348,1927$.

43. Berenson, G.S. Distribution of acid mucopolysaccharide in inner and outer layers of bovine aorta. Circ. Res. 7: 889,1959 .

44. Bergel, H.D. The thickness of the arterial wall. J. Physiol. 150: 10P, 1960.

45. Bergel, D.H. The static elastic properties of the arterial wall. J. Physiol. 156: 445, 1961.

46. Bertelsen, S. and C.E. Jensen. Histochemical studies on human aortic tissue. Acta Pathol. Microbiol. Scand. 48: 305,1960 .

47. Bevan, J.A. and M.A. Verity. Postganglionic sympathetic delay in vascular smooth muscle. J. Pharm. Exp. Therap. 152: 221,1966 .

48. Bloom; W. and R.H. Sandstrom. Development of connective tissue fibers in epithelium-containing cultures. Anat. Rec. 64: 75,1935 . 
49. Boeke, J. The sympathetic end-formation, its synaptology, the interstitial cells, the periterminal netuork, and its bearing on the neurone theory. Discussion and critique. Acta Anat. 8: 18, 1949.

50. Bohr, D.F., R.S. Filo, and K.F. Guthe. Contractile protein in vascular smooth muscle. Physiol. Rev. 42: 98, 1962 (Suppl. 5, part II).

51. Bolduan, O.E.A. And R.S. Bear. Unidirectional nature of the large periodic structure of collagen fibrils. J. Polymer Sci. 5: 159, 1950.

52. Bolduan, O.E.A. and R.S. Bear. Ceneral nature of the extension of structure transverse to collagen fibril axes. J. Polymer Sci. E: 271, 1951.

53. Boss, Je and J.H. Green. The histology of the common carotid baroreceptor areas in the cat. Circ. Res. 4: $12,1956$.

54. Bowes, J.H. and R.H. Kenten. Some observations on the amino. acid distribution of collagen, elastin and reticular tissue from different sources. Biochem. J. 45: 281, 1949 .

65. Bozler, E. An analysis of the properties of smooth muscle. Cold Spr. Harb. Symp. Quant. Biol. 4: 260, 1936.

56. Bozler, E. Extensibility of contractile elements. In, Tissue Elasticity. J.W. Remington, ed. Wastington, D.C.: Am. Physiol. Soc., 1957. $102 \mathrm{p}$.

57. Bramwell, J.C., A.C. Downing and A.V. Hill. The effect of blood pressure on the extensibility of the human artery. Heart 10: 289, 1923a.

58. Bramwell, J.C., R.J.S. McDowall, and B.A. Mcswiney. The variation of arterial elasticity with blood pressure in man. Proc. Roy. Soc. (London) B 94: 450, 1923b.

59. Briggs, A.H. Characteristics of contraction in glycerinated uterine smooth muscle. Am. J. Physiol. 204: 739, 1963.

60. Briggs, F.N. and F. Fuchs. The biosynthesis of a muscle relaxing substance. Biochem. Biophys. Acta 42: $519,1960$. 
61. Brocklehurst, R.J. Studies on the physiology of plain muscle. The effect of alteration of initial length on the tension produced on contraction., J. Physiol. 61: 275,1926 .

62. Buchthal, F. and T. Weis-Fogh. Contribution of the sarcolemma to the force exerted by resting muscle of insects. Acta Physiol. Scand. 35: 345, 1956.

63. Buck, R.C. Fine structure of endothelium of large arteries. J. Biophys. Biochem. Cytol. 4: 187, 1958.

64. Buddecke, E. Untersuchungen zur Chemie der Arterienwand, V. Darstellung und Chemische Zusammensetzung von Mucopolysacchariden der Aorta des Menchen. Z. Physiol. Chem. Hoppe-Seyler's 318: 33, 1960.

65. Bulbring, E. Measurements of oxygen consumption in smooth muscle. J. Physiol. 122: 111, 1953.

66. Bulbring, E. Membrane potentials of smooth muscle fibers of the taenia coli of the guinea pig. J. Physiol. 125: $302,1954$.

67. Bulbring, E. and H. Kuriyama. Effects of changes in the external sodium and calcium concentrations on spontaneous electrical activity in smooth muscle of guinea pig taenia coli. J. Physiol. 166: 29, 1963.

68. Bulbring, E. and N.H. Lullman. The effect of metabolic inhibitors on the electrical and mechanical activity of the smooth muscle of the guinea pig's taenia coli. J. Physiol. 136: 310, 1957.

69. Bunce, D.F.M., II. Structure differences between distended and collapsed arteries. Angiology 16: 53, 1965.

70. Burnstock, G. and C.L. Prosser. Responses of smooth muscles to quick stretch; relation of stretch to conduction. Am. J. Physiol. 198: 921, 1960.

71. Burton, A.C. The physical equilibrium of small blood vessels. Am. J. Physiol. 164: 319, 1951. 
72. Burton, A.C. Relation of structure to function of tissue of the wall of the blood vessels. Physiol. Rev. 34: $619,1954$.

73. Burton, D., D.A. Hall, M.K. Keech, R. Reed, H. 'Saxl, R.E. Tunoridge, and $\mathrm{m.J}$. Wood. Apparant transformation of collagen fibrils into elastin. Nature 176: $966,1955$.

74. Caeser, R., G.A. Edwards, and H.J. Ruska. Architecture and nerve supply of mammalian smooth muscle tissue. J. Biophys. Biochem. Cytol. 3: 867, 1957.

75. Carew, T.E., R.N. Vaishnav, and D.J. Patel. Compressibility of the arterial wall. Fed. Proc. 27: 74, 1968.

76. Carlson, F, G.G. Knuppeis, and F. Buchtal. Ultrastructure of the resting and contracted striated muscle fiber at different degrees of stretch. J. Biophys. Biochem. Cytol. 11: 95, 1961.

77. Carton, R.W., J. Dainauskas, and J.W. Clark. Elastic properties of single elastic fibers. J. Appl. Physiol. 17: 547,1962 .

78. Casella, C. Tensile force in total striated muscle, isolated fiber and sarcolemma. Acta Physiol. Scand. 21: 380.1950 .

79. Cater, T.J.. and J.R. Kanagy. A flex test for leather. J. Am. Leath. Chem. Assoc. 49: 23, 1954.

80. Chain, E. and E.S. Duthie. A mucolytic enzyme in testis extracts. Nature 144: 977, 1939.

81. Cohnheim, 0 . and J. von Uexküll. Die Dauerkontraktion der glutten Muskeln. Ztschr. f. Physiol. Chem. 76: 314, 1911.

82. Conabere, G.0.. and R.H. Hall. Physical properties of individual leather fibers. I. Evaluation of Young's modulus of elastic extension and percentage permanent set of leather fibers. J. Int. Soc. Leath Tr. Chem. 30: : $214,1946$. 
(183. Conti, G., B. Haenni, L. Laszt and C. Rouiller. Structure et ultrastructure de la cellule musculaire lissa de la paroi carotidienne a l'etat de repos et a l'etat de contraction. Angiologica 1: 119, 1964.,

84. Conway, E.J. Nature and significance of concentration relations of potassium and sodium ions in skeletal muscle. Physiol. Rev. 37: 84, 1957.

85. Corrodi, H., N-A Hillarp and G. Jonsson. Fluorescence methods for the histochemical demonstration of monoamines. 3. Sodium borohydride reduction of the fluorescence compounds as a specificity test. J. Histochem. Cytochem. 12: 582, 1964.

86. Cowan, P.M., A.C.T. North, and J.T. Randall. X-ray diffraction studies of collagen fibers, in fibrous proteins and their biological significance. Symp. Soc. Exptl. Biol. 9: 115, 1955.

87. Cox,R.C. and K. Little. An electron microscope study of elastic tissue. Proc. Roy. Soc. Biol. 155: 232, 1961.

88. Csapo, A. and M. Goodall. Excitability, length-tension relation and kinetics of uterine muscle contraction in relation to hormonal status. J. Physiol. 126: 384, 1954.

89. Curran, R.C. The elaboration of mucopolysaccharides by vascular endothelium. J. Path. Bacteriol. 74: 347, 1957.

90. Curran, R.C. and J.S. Kennedy. Utilization of sulphate ion by fibroblasts in quartz focus. Nature 175: 435, 1955.

91. Daemers-Lambert, C. and J. Roland. Metabolisme des esters phosphores pendant le developpement et le maintien de la tension phasique de muscle lisse arteriel (carotides de bovide) Angiologica 4: 69, 1967.

92. Dahl, E. and E. Nelson. Electron microscopic observations on human intracranial arteries. Arch. Neurol. \& Psychiat. 10: 158, 1964. 
93. Davies, D.V. Observations on the volume viscosity and nitrogen content of the synovial membrane. J. Anat. 78: 68,1944 .

94. Davignon, J., R.R. Lorenz, and J.T. Shepherd. Response of human umbilical artery to changes in transmural pressure. Am. J. Physiol. 209: 51, 1965.

95. Davis, D. Sympathetic stimulation and small artery constriction. Am. J. Physiol. 206: 262, 1964.

96. delastro, $F$. Sur la structure et l'innervation du sinus carotidien de l'homme et des mammiferes. Nouveaux faits sur l'innervation et la fonction du glomus caroticum. Etudes anatomiques et physiologiques. Trab. Lab. Invest. Biol. Univ. Madr. 25: 331, 1928.

97. Dees, M.b. On the fenestrated membrane of Henle. Anat. Rec. 26: $169,1923$.

98. Dettmer, N. Elektronenmikroskopische untersuchungen am elastischen Fasersystem des Ligamentum Nuchae. Z. Zellforsch. Mikroskop. Anat. 45: 265, 1956.

99. DeVillafranca, G.W. and D.C. Naumann. Some properties of myosin B ATP-ase from Limulus. Compar. Biochem. Physiol. 12: 143, 1964 .

100. Ducret, S. Die Dehnungsegenschaften der Coronar und Splancknicus arterian. Pflug. Arch. Ges. Physiol. 225: 669,1930 .

101. Ducret, S. Rhythmische Tonusschwankungen und Adrenalinerrgbarkeit der mesenterialgefasse. Pflug. Arch. Ges. Physiol. 227: 753, 1931.

102. Durbin, R.P. and R.R. Monson. Ionic composition and permeability of smooth muscle. Fed. Proc. 20: 134, 1961.

103. Eastor, J.E. The amino acid composition of mammalian collagen and gelatin. Biochem. J. 61: 589, 1955.

104. Ebel, A. and R. Fontaine. Le collagene et hydroxyproline dans la paroi aortique des bovides ot sos variations an cours du viellissement. Path. Biol. 12: 242, 1964. 
(175. Elliott, G.F. X-ray diffraction studies on striated and smooth muscle. Proc. Roy. Soc. Lon., Series $B$, 160: 467,1964 .

106. Elliott, G.F. Variations of the contractile apparatus in smooth and striated muscles. X-ray diffraction studies at rest and in contraction. J. Gen. Physiol. 50: 171,1966 .

107. Evans, C.L. Studies on the physiology of plain muscle II. The oxygen usage of plain muscle, and its relation to tonus.. J. Physiol. 58: 22, 1923.

108. Evans, C.L. and A.V. Hill. The relation of length to tension development and heat production on contraction in muscle. J. Physiol. 49: 10, 1914.

109. Evans, D.H.L. and E.M. Evans. The membrane relationships of smooth muscles: An electron microscope study. J. Anat. 98: 37,1964.

110. Evans, D.H.L., H.O. Schild, and S. Thesleff. Effects of drugs on depolarized plain muscle. J. Physiol. 143: 474,1958 .

11. Faber, M. and G. Moller-Hou. The human aorta. V. Collagen and elastin in the normal and hypertensive aorta. Acta Path et Microbiol. Scand. 31: 377, 1952.

112. Falck, B. Observations on the possibilities of the cellular localization of monoamines by a fluorescence method. Acta Physiol. Scand. (Suppl. 197) 56: 1, 1962.

113. Falck, B., N-A. Hillarp, G. Theime, and A. Torp. Fluorescence of catecholamines and related compounds condensed with formaldehyde. J. Histochem. Cytochem. 10: 348,1962 .

114. Fang, T.P. The effect of length on the resting metabolism of muscle. J. Physiol. 74: 441, 1932.

115. Fenn, W.0. Some elasticity problems in the human body. In, Tissue Elasticity, J.W. Romington, ed. Washington, D.C.8 Am. Physiol. Soc., 1957. $154 \mathrm{P}$. 
) f(116. Ferrara, A. Sulle possibilita evolutive embolastiche dagli endoteli vascolari. II. Risultanze sperimentali, deduzioni interpretative e conclusione. Arch. "de Yecchi" Anat. Pat. Med. 14: 193, 1950.

117. Filo, R.S., J.C. Ruegg, and D.F. Bohr. Actomyosin-like protein of arterial wall. Am. J. Physiol. 205: $1247,1963$.

118. Fischer, G.M. and J.G. Llaurado. Collagen and elastin contont in canine arteries selected from functionally different vascular beds. Circ. Res. 19: 394, 1966.

119. Fischer, G.M. and J.G. Llaurado. Connective tissue composition of canine arteries. Arch. Pathol. 84: $95,1967$.

120. Fischer, H. Uber die funktionelle Bedeutung des Spiralverlaufes der Muskulatur in der Arterienwand. Morph. Jahrb. 91: 395, 1951.

121. Franchini, C.M. and E. DeRobertis. Electron microscopo observations on elastic fibers. Proc. Soc. Exp. Biol. Med. 76: 515, 1951.

122. Fujino, M., K. Kasai, and K. Koseki. The effect of temperature on actomyosin adenosinetriphosphatase activity and superprecipitation. Jap. J. Physiol. 9: $228,1959$.

123. Funaki, S. Spontaneous spike-discharges of vascular smooth muscle. Nature 191: 1102, 1961.

124. Fung, Y.C., B.W. Zweifach, and M. Intaglietta. Elastic environment of the capillary bed. Circ. Res. 19: $441,1966$.

125. Furchgott, R.F. and E. Shorr. Sources of energy for intestinal smooth muscle contraction. Proc. Soc. Exp. Biol Med. 61: 280, 1946 .

126. Fuxe, K. and G. Sedvall. The distribution of adrenergic nerve fibers to the blood vessels in skeletal muscle. Acta Physiol. Scand. 64: 75, 1965. 
127. Fyfe, F.W. and T. Gillman. Electron microscopy of commencing elastogenesis in embryonic mouse aorta. J. Anat. 98: 682, 1964 .

128. Gaspar-Godfroid, A. L'activite adenosinetriphosphatasique de la tonomyosine de carotides de bovide. Angiologica 1: 12, 1964.

129. Geiringer, E. Intimal vascularization and atheromatosis. J. Pathol. \& Bact. 63: 201, 1951.

130. Gerova, $M$. and J. Gero. Reflex regulation of smooth muscle tone of conduit vessel. Angiologica 4 : $348,1967$.

131. Cersh, I. and H.R. Catchpole. The organization of ground substance and basement membrane and its significance in tissue injury, disease and growth. Am. J. Anat. 85: 457,1949 .

132. Gibson, T., R.m. Kenedi, and J.E. Craik. The mobile microarchitecture of dermal collagen. Brit. J. Surg. 52: 764,1965 .

133. Gillespie, J.S. An isolated innervated artery preparation. J. Physiol. 187: 2, 1966.

134. Coerttler, $K$. Über den Einbau der grossen Venen des menschlichen Unterschenkels. Ztschr. Anat. u. Entwick. 116: 591,1953 .

135. Goodford, P.J. and K. Hermansen. Sodium and potassium movements in the unstriated muscle of the guinea pig. taenia coli. J. Physiol. 158: 426, 1961.

136. Core, I. and B.J. Larkey. Functional activity of aortic mucopolysaccharides. J. Lab. Clin. Med. 56: 839, 1960.

137. Goto, $m$. and $Y$. Kimoto Hysteresis and stress-relaxation of the blood vessels studied by a universal tensile testing instrument. Jap. J. Physiol. 15: 169, 1966.

138. Gotte, L. and A. Sarafini-Fracassini. Electron microscope observations on the structure of elastin. J. Atherosclor. Res. 3: 247, 1963. 
179. Greenfiold, J.C., W.G. Austen, and D.J. Patel. Mechenics of the human femoral artery in vivo. Angiology 16: $460,1965$.

140. Greenlee, T.K., Jr., R. Russell, and J.L. Hartmen. The fine structure of elastic fibers. J. Cell. diol. 30: $59,1966$.

141. Gross, J. The structure of elastic tissua as studied with tho electron microscope. J. Exptl. Med. 89: 699, 1949 .

142. Grossfield, H., K. Meyer, and G.C. Godman. Differentiation of fibroblasts in tissue culture, as determined by mucopolysaccharide production. Proc. Soc. Exp. Biol. Med. 88: $31,1955$.

143. Grunmach, E. Ueber die Beziehung der Dehnungscurve elastischer Rohren zur Pulsgeschwindigkeit. Arch. f. Anat. U. Physiol. 12: 129, 1883.

144. Gustavson, K.H. The Chemistry and Reactivity of Collagen. Now York: Academic Press, 1956.

145. Guth, E. Muscular contraction and rubber-like elasticity. Ann. N.Y. Acad. Sci. 47: 735, 1947.

Haddy, F.J., M. Fleishman, and D.A. Emanusl. Effact of opinophrino, norepinephrine and serotonin upon systemic small and large vessel resistance. Circ. Res. 5: 247, 1957a.

147. Haddy, F.J., fi. Fleishman, and J.B. Scott, Jr. Effect of change in air temperature upon systemic small and large vessel resistance. Circ. Res. $\underline{5}$ : 58, 1957b.

148. Hall, D.A. Studies on the complex nature of the elastinelastase system. Biochem. J. 55: XXXV, 1953.

149. Hall, D.A. and J.W. Czerkawski. The reaction between elastase and elastic tissue. Biochem. J. 80: 134, 1961.

150. Hall, D.A., R. Reed, and R.E. Tunbridge. Structure of elastic tissue. Nature 170: 264, 1952.

151. Hall, D.A., R. Reed, and R.E. Tunbridge. Electron microscope studies of elastic tissue. Exp. Cell Res. 8: $35,1955$. 
152. Hall, R.H. Changes in length of stressed collagen fibers with time. J. Soc. Leath. Tr. Chem. 35: 11, 1951a.

153. Hall, R.H. Variations with $\mathrm{pH}$ of the tensile properties of collagen fibers. J. Soc. Leath. Tr. Chem. 35: 195, 1951b.

154. Hallock, P. and I.C. Benson. Studies on the elastic properties of human isolated aorta. J. Clin. Invest. 16: $595,1937$.

155. Hamoir, G. and L. Laszt. Tonomyosin of arterial muscle. Nature 193: 682, 1962.

156. Hampel, C.w. The effect of initial tension and load on the response of the nictitating membrane of the cat.. Am. J. Physiol. 107: 717, 1934.

157. Hanson, J. and J. Lowy. The structure of the muscle fibers in the translucent part of the adductor of the oyster Crossostrea angulata. Proc. Roy Soc. (London) B 154: 173, 1961.

158. Hanson, $J_{\text {. }}$ and J. Lowy. Discussion follousing paper by D.M. Needham and C.F. Schoenberg. Proc. Roy. Soc. (London) 8 160: 523, 1964.

159. Hardung, V. Vergleicheude Messungen der dynamischen Elastizitat and Viskositat von Blutgefassen, Kautschauk und synthetischen Elastomeren. Helvet. Physiol. et Pharm. Acta 11: 194, 1953.

160. Harkness, R.D. Biological functions of collagen. Biol. Rev. 36: 399, 1961.

161. Harkness, M.L.R., R.D. Harkness, and D.A. McDonald. The collagen and elastin content of the arterial wall in the dog. Proc. Roy. Soc. (London) a 146: 541, 1957.

162. Hass, G.M. Elastic tissue. II. A study of the elasticity and tensile strength of elastic tissue isolated from the human aorta. Arch. Path. 34: 971, 1942.

163. Hayes, J.R. Histological changes in constricted arteries and arterioles. J. Anat. 101: 343, 1967. 
164. Herringham, W.P. and W.A. Wills. On the elasticity of the aorta, being a contribution to the study of arterial sclerosis. Trans. Roy. Med.-Chir. Soc. 89: 489, 1904.

165. Hill, A,V, The viscous elastic properties of smooth muscle. Proc. Roy. Soc. (London) B 100: 100, 1926.

166. Hillarp, N. The construction and functional organization of the autonomic innervation apparatus. Acta Physiol. Scand. 46: 1, 1959 (Suppl. 157).

167. Hinke, J.A.M. and M.L. Wilson. A study of elastic properties of a 550- $\mu$ artery in vitro. Am. J. Physiol. 203: 1153, 1962 .

168. Hirsch, C. Contribution to the pathogenesis of chondromalacia of the patella; physical, histologic and chemical study. Acta Chir. Scand. 90: 1, 1944 (Suppl. 83).

169. Hochrein, M. and B. Singer. Untersuchungen am venösen Teil des Kreislaufes. II. Mitteilung: Untersuchungen Uber den Bau der Veneusand. Arch. f. Exptl. Path. u. Pharm. 125: 301, 1927.

170. Hoeve, C.A. and P.J. Flory. The elastic properties of elastin. J. Am. Chem. Soc. 80: 6523, 1958.

171. Holman, M.E. Membrane potentials recorded with high-resistance microelectrodes and the effects of changes in ionic environment on the electrical and mechanical activity of the smooth muscle of the taenia coli of the guinea pig. J. Physiol. 141: 464, 1958.

172. Horn, H. and L.E. Finklestein. Arteriosclerosis of the coronary arteries and the mechanism of their occlusion. Am. Heart. J. 19: 655, 1940.

173. Hosoda, $Y$. and I. Minoshima. Elastin content of the aorta and the pulmonary artery in the Japanese. Angiology 16: 325,1965 .

174. Huber, G.C. A note on the structure of the elastica interna of arteries. Anat. Rec. 11: 169, 1916.

175. Hume, J.F. The tensility of the rat's aorta as influenced by age, environmental temperature and certain toxic substances. Am. J. Hyg. 29: 11, 1939. 
176. Hürthle, K. "Uber die Beziehung zwischen Durchmesser und Wandstarke der Arterien nebst Schatzung des Anteils der einzelnen Gewebe am Aufban der Wand. Pflug. Arch. ges Physiol. 183: 253, 1920.

177. Hürthle, K. Vergleich der Druck-und Durchmesserschwankungen der Arterien. Pflug. Arch. ges Physiol. 200: $49,1923$.

178. Huxley, H.E. Muscle cells. In The Cell, Vol. IV, J. Brachet and A.E. Mirsky, ed. New York: Academic Press, 1960. Chapter ?.

179. Huxley, H.E. Electron microscope studies on the structure of natural and synthetic protein filaments from striated muscle. J. Mol. Biol. I: 281, 1963.

180. Israel, 0. Heber erwonbene Störungen in den Elasticitätsverhaltnissen der Grossen Gefasse Virchow's. Arch. f. Pathol. Anat. U. Physiol. 103: 461, 1886.

181. Jaeger, $M$. The flow through the arterial wall. In Pulsatile Blood Flow. E.O. Attinger, ed. New York: McGraw-Hill, 1964. $307 \mathrm{p}$.

182. Jensen, J.G. An electron microscopic study on the morphology of the elastin in foetal human aorta. Acta Path. et Microbiol. Scand. 56: $388,1962$.

183. Johnson, W.H. Tonic mechanisms in smooth muscles. Physiol. Rev. 42: 113, 1962. (Suppl. 5, part II).

184. Johnson, W.H. and J. Garber. The length dependence of stretch resistance during the catch state in molluscan muscles. J. Gen. Physiol. 50: 2502, 1967.

185. Kaplan, D. and K. Meyer. Mucopolysaccharides of the aorta at various ages. Proc. Soc. Exp. Biol. Med. 105: $78,1960$.

186. Kappeler, K. and D. Mayor. Accumulation of noradrenaline proximal to the site of constriction of sympathetic nerves. J. Physiol. 182: 44P, 1966a. 
187. Kappeler, K. and D. Mayor. Ultrastructural changes proximal to a constriction in sympathetic nerves during the first 24 hours after operation. J. Anat. 100: $439,1966 b$.

188. Karrer, H.E. The fine structure of connective tissue in taenia propria of bronchioles. J. Ultrastruct. Res. 2: 96,1958 .

189. Karrer, H.E. An electron microscopic study of the aorta in young and in aging mice. J. Ultrastruct. Res. $\underline{5}$ : $1,1961$.

190. Katz, L.N., M.R. Malinow, B. Kondo, D. Feldman, and N. Grossman. The volume elasticity of the aorta in the intact dog. Am. Heart J. 33: $319,1947$.

191. Keatinge, W.R. Mechanisms of adrenergic stimulation of mammalian arteries and its failure at low temperatures. J. Physiol. 174: 184, 1964.

192. Keatinge, W.R. Electrical and mechanical responses of vascular smooth muscle to vasodilator agents and vasoactive polypeptides. Circ. Res. 18: 641, 1966а.

193. Keatinge, W.R. Electrical and mechanical response of arteries to stimulation of sympathetic nerves. J. Physiol. 185: 701, 1966b.

194. Keech, M.K. Electron microscope study of the normal rat aorta. J. Cytol. I: 533, 1960.

195. Kenedi, R.M., C.H. Daly, and T. Gibson. The determination, significance and application of the biomechanical characteristics of human skin. Digest of the 6 th International Conference on Medical Electronics and Biological Enoineering. Tokyo, 1965. $531 \mathrm{p}$.

196. Kennedy, J.J. Tubular structure of collagen fibrils. Science 121: 673, 1955.

197. Kennedy, J.S. Sulphur 35 in connective-tissue formation. J. Pathol. Bact. 80: 359, 1960.

198. Kimoto, $Y$. and $M$. Goto. The effect of temperature on tension, hysteresis loop and stress-relaxation of the blood vessels. Jap. J. Physiol. 17: 356, 1967. 
199. Kirk, J.E. and T.J.S. Laursen. Diffusion coefficients of various solutes for human aortic tissue. J. Gerontol. 10: 288, 1955.

200. Kolpak, H.V. Röntgenstrukturuntersuchungen "Wter elastisches Gewebe unter besondever Berucksichtigung der Dehnung und Entquallung. Kolloid. Ztschr. 73: 129, 1935.

201. Kosan, R.L. and A.C. Burton. Uxygen consumption of arterial smooth muscle as a function of active tone and passive stretch. Circ. Res. 18: 79, 1966.

202. Kraemer, D.M. and H. Miller. Elastin content of the albuminoid fraction of human aorta. Arch. Path. 55: $70,1953$.

203. Krafka, J. Comparative study of the histophysics of the aorta. Am. J. Physiol. 125: 1, 1939.

204. Kuriyama, $H$. The influence of potassium, sodium and chloride on the membrane potential of the smooth muscle of taenia coli. J. Physiol. 156: 15, 1963.

205. Kuriyama, $H$. and $T$. Tomita. The responses of single smooth muscle cells of guinea pig taenia coli to intracellularly applied currents, and their effect on spontaneous electrical activity. J. Physiol. 178: 270, 1965.

206. Labella, F.R. Elastin, a metabolically active lipoprotein. Nature 180: 1360, 1957.

207. Laitinen, E.A. Über die Struktur elastischer Fasern und ihre Beziehungen zu den Kollagenfibrillen. Ztschr. Anat. Entwick. 121: 388, 1960.

208. Landgren, S. The baroreceptor activity in the carotid sinus nerve and the distensibility of the sinus wall. Acta Physiol. Scand. 26: 35, 1952.

209. Lane, B.P. Alterations in the cytological detail of intestinal smooth muscle cells in various stages of contraction. J. Cell. Biol. 27: 199, 1965. 
210. Lansing, A.I., T.B. Rosenthal, M. Alex, and E.W. Dempsey. The structure and chemical characteristics of elastic fibers as rovealed by olastase and by electron microscopy. Anat. Rec. 114: 555, 1952.

211. Laszt, L. Properties of vessel muscle proteins extracted with water or salt solutions of low ionic strength. Nature 189: 230, 1961.

212. Laszt, L. Was ist Gefastonus? Angiologica 1: 346, 1964. 213, Laszt, L. Untersuchungen uber die elastischen Eigenschaften der Blutgefase im Ruhe - und im Kontraktionszustand. Angiologica 5 : 14,1968 .

214. Lawton, R.W. The thermoelastic behavior of isolated aortic strips of the dog. Circ. Res. $4: 344,1954$.

215. Lawton, R.W. Somo aspects of research in biological elasticity. Introductory remarks. In Tissue Elasticity. J.W. Remington, ed. Washington, D. C.: Am. Physiol. Soc., 1957, p.11.

216. Learoyd, B.M. and M.G. Taylor. Alterations with age in the viscoelastic properties of human arterial walls.

Circ. Res. 18: 278, 1966.

217. Leary, T. Vascularization of arteriosclerotic lesions. Am. Heart J. 16: 549, 1938 .

218. Lee, K.S., H. Ladinsky, S.J. Choi, and Y. Kasuya. Studies on the in vitro interaction of electrical stimulation and $\mathrm{Ca}+$ movement in sarcoplasmic reticulum. J. Gen. Physiol. 49: 689, 1966.

219. Leonard, E. and S.J. Sarnoff. Effect of aramine-induced smooth muscle contraction on length-tension diagrams of venous strips. Circ. Res. $5:$ 169, 1957.

220. Lever, J.D., J.D.P. Graham, G. Irvine, and W.J. Chick. The vesiculated axons in relation to arteriolar smooth muscle in the pancreas. A fine structure and quantitative study. J. Anat. 99: 299, 1965.

221. Linden, I.H., E. Laden, J.0. Erickson, and D. Armen. Electron microscopic study of normal skin and collagen fibers. J. Invest. Derm. 24: 83, 1955. 
222. Ling, G. and R.W. Gerard. External potassium and the membrane of single muscle fibers. Nature 165: 113, 1950.

223. Lloyd, O.J., L.D. Jordan, and M. Garrod. A contribution to tho theory of the structure of protein fibers with. special reference to the so-called thermal shrinkage of the collagen fiber. Tr. Faraday Soc. 64: 441, 1948 .

224. Loeven, W.A. Elastolysis. Remarks on some properties of elastomucase and elastoproteinase purified by means of starch column electrophoresis. Acta Physiol. Pharm. Neerl. 9: 473, 1960.

225. Low, F.N. Microfibrils: Fine filamentous components of the tissue space. Anat. Rec. 142: 131, 1962.

226. Lowry, O.H., D.R. Gilligan, and E.M. Katersky. The determination of collagen and elastin in tissues, with results obtained in various normal tissues from different species. J. Biol. Cher. 139: 795, 1941.

227. Lowy, J. and B.M. Millman. Contraction and relaxation in smooth muscle of lamellibranch-molluscs. Nature 183: 1730, 1959 .

228. Lundholm, L. and E. Mohme-Lundholm. The carbohydrate metabolism and tone of smooth muscle. Acta Pharm. et Toxicol. 16: $374,1960$.

229. Lundholm, L. and E. Mohme-Lundholm. Studies on the effects of drugs upon the lactic acid metabolism and contraction of vascular smooth muscle. Acta Physiol. Scand. 55: 45,1962 .

230. Lundholm, L. and E. Mohme-Lundholm. Energetics of isometric and isotonic contraction in isolated vascular smooth muscle under anaerobic conditions. Acta Physiol. Scand. 64: 275, 1965.

231. Lundholm, L. and E. Mohme-Lundholm. Length at inactivated contractile elements, length-tension diagram, active state and tone of vascular smooth muscle. Acta Physiol. Scand. 68: 347, 1966.

232. McCallum, H.M. Experimental lathyrism in mice. J. Path. Bact. 89: $625,1965 a$ 
233. McCallum, H.M. Experimontal lathyrism in tissue culture. J. Path. Bact. 89: 637, 1965b.

234. McCartney, J.E. Heat contraction of elastic/tissue. Quart. J. Exptl. Biol. 2: 103, 1913.

235. MeDonald, D.A. Blood Flow in Arteries. London: E. Arnold, Ltd., 1960. $32 \overline{\mathrm{p}}$.

236. Macht, D.I. The action of diugs on the isolated pulmonary artery. J. Pharm. Exptl. Therap. E: 13, 1914.

237. Mecill, C. The structure of smooth muscle in the resting and the contracted condition. Am. J. Anat. 9: 493, 1909 .

238. Maclvilliam, J.A. On the properties of the arterial and venous walls. Proc. Roy. Soc. (London) 70: 109, 1902.

239. MacWilliam, J.A. and A.H. mackie. Observations on arteries, normal and pathological. Brit. Med. J. ii: 1477 1908 .

240. Maeser, $m$. The resistance of leather to flexural fatigue. J. Am. Leath. Chem. Assoc. 39: 35, 1944.

241. Marey, Traveaux de Laboratoire $4: 178,1880$.

242. Mark, J.S.T. An electron microscope study of uterine smooth muscle. Anat. Fec. 125: 473, 1956.

243. Matthews, M.A. and D.L. Gardner. The fine structure of the mesenteric arteries of the rat. Angiology 17: 902, 1966.

244. Meyer, K.H. and C. Ferri. Die elastischen Eigenschaften der elastischen und der kollageneu fasern und ihre molekulare Deutung. Pflug. Arch. ges Physiol. 238: 78,1937 .

245. Mitton, R.G. and F.R. Morgan. The mechanical properties of vegetable tanned collagen fibers. J. Soc. Leath. Tr. Chom. 44: 58,1960 .

246. Morgan, F.R. The mechanical properties of collagen fibers: stress-strain curves. J. Soc. Leath. Tr. Chem. 44: $170,1960$. 
247. Movat, M.Z. and N.V.P. Fernando. The fine structure of the terminal vascular bed. I. Small arteries with an internal elastic lamina. Exptl. \& Molec. Path. 2 $549,1963$.

248. Muscatello, U., E. Andersson-Cedergren, and G.F. Azzone. The mechanism of muscle-fiber relaxation adenosine triphosphatase and relaxing activity of the sarcotubular system. Biochem. Biophys. Acta 63: 55, 1962.

249. Mustacchi, P.I. Extensibility of hydrated collagen. J. Am. Leath. Tr. Chem. Assoc. 46: 479, 1951.

250. Myers, V.C. and W.W. Lang. Some chemical changes in human thoracic aorta accompanying the aging process. J. Gerontol. 1: 441, 1946.

251. Needham, D.M. and C.F. Shoenberg. Proteins of the contractile mechanism of mammalian smooth muscle and their possible location in the cell. Proc. Roy. Soc. (London) B 160: 517, 1964 .

252. Needham, D.M. and J.M. Williams. Proteins of the uterine contractile mechanism. Biochem. J. 89: 552, 1963.

253. Nelson, M.G. Intimal coronary artery hemorrhage as a factor in the causation of coronary occlusion. J. Path. \& Bact. 53: 105,1941 .

254. Neukirch, $P$. and $P$. Rona. Experimentelle Beiträge zur Physiologie des Darmes I. Pflug. Arch. ges. Physiol. 144: 555,1912 ,

255. Nouman, R.E. The amino acid composition of gelatins, collagens, and elastins from different sources. Arch. Biochem. 24: 289, 1949 .

256. Neuman, R.E. and M.A. Logan. The determination of collagen arid elastin in tissues. J. Biol. Chem. 186: 549, 1950.

257. Norberg, K. and B. Hamberger. The sumpathetic adrenergic neuron. Acta Physiol. Scand. 63(Suppl.): 238, 1964.

258. Nutting, G.C. and R. Borasky. Electron microscopy of collagen. J. AM. Leath Chem. Assoc. 43: 96, 1948. 
259. D'Dell, B.L. D.F. Elsden, J. Thomas, S.M. Partridge, R.H. Smith, and R. Palmer. Inhibition of the biosynthesis of the cross-links in elastin by a lathyrogen. Nature 209: 401, 1966.

260. O'Dell, B.L., B.C. Hardujick, G. Reynolds, and J.E. Savage. Connective tissue defect in the chich resulting from copper deficiency. Proc. Soc. Exp. Biol. Med. 108: $402,1961$.

261. Palmer, R.F. and V.A. Posey. Ion effects on calcium accumulation by cardiac sarcoplasmic reticulum. J. Gen. Physiol. 50: 2085, 1967.

262. Panner, B.J. and C.R. Honig. Filament ultrastructure and organization in vertebrate smooth muscle. J. Cell. Biol. 35: 303, 1967 .

263. Parker, F. An electron microscope study of coronary arteries. Am. J. Anat. 103: 247, 1958.

264. Parker, G.H. The production of carbon dioxide by the smooth muscle of sea-anemones. Am. J. Physiol. $\underline{59}$ : $466,1922$.

265. Partridge, S.M. Diffusion of solutes in elastin fibers. Biochem. et Biophys. Acta 140: 132, 1967.

266. Partridge, S.M. and H.F. Davis. The chemistry of connective tissues. 3. Composition of the soluble proteins derived from elastin. Biochem. J. 61: 21, 1955.

267. Partridge, S.M., D.F. Elsden, and j. Thomas. Constitution of the cross-linkages in elastin. Nature 197: $1297,1963$.

268. Partridge, S.M., D.F. Elsden, J. Thomas, A. Dorfman, A. Telser, and Peri-Lee Ho. Biosynthesis of the desmosine and isodesmosine cross-bridges in elastin. Biochem. J. 93: 30C, 1964.

269. Partridge, S.M., D.F. Elsoden, J. Thomas, A. Dorfman, A. Telser, and Peri-Lee Ho. Incorporation of labelled lysine into the desmosine cross-bridges in elastin. Nature 209: 399, 1966. 
270. Patel, D.J., A.J. Mallos, and D.L. Fry. Aortic mechanics in the living dog. J. Appl. Physiol. 16: 293, 1961.

271. Patel, D.J., D.P. Schilder, and A.J. Mallos. Mechanical properties and dimensions of the major pulmonary arteries. J. Appl. Physiol. 15: 92, 1960.

272. Paterson, J.C. Vascularization and hemorshage of the intima of arteriosclerotic coronary arteries. Arch. Path. 22: 313,1936 .

273. Peachey, L.D. and K.R. Porter. Intracellular impulse conduction in muscle cells. Science 129: 721, 1959.

274. Pease, D.C., and W.J. Paule. Electron microscopy of elastic arteries; the thoracic aorta of the rat. J. Ultrastruct. Res. 3: $469,1960$.

275. Pease, D.C. and S. Molinari. Electronmicroscopy of muscular arteries: Pial vessels of the cat and monkey. J. Ultrastruct. Res. 3: 447, 1960.

276. Peterson, L.H. Physical factors which influence vascular caliber and blood flow. Neurohumoral mechanisms and vascular smooth muscle. Circ. Res. (Suppl. 1) 18: $1,1966$.

277. Peterson, L.H., R.E. Jensen, and J. Parnell. Mechanical properties of arteries in vivo. Circ. Res. 8: 622, 1960.

278. Piez, K.A. and J. Gross. The amino acid composition of some fish collagens: The relation between composition and structure. J. Biol. Chem. 235: 995, 1960.

279. Policard, A., A. Collet, and L. Ciltaire-Ralyte. Observations au microscope electronique sur la structure inframicroscope des artérioles des mammifores. Bull. Microscop. Appl. $\underline{5}:$ 3, 1955.

280. Policard, A., A. Collet, and S. Pregermain. Observation au microscope electronique sur quelques voisseaux pulmonaires. Bull. Microscop. Appl. 10: 17, 1960.

281. Polley, E.H.. Tho innorvation of blood vossols in striated musclo and skin. J. Comp. Neurol. 103: 253, 1955. 
282. Polotebnow. Sklerose des arteriellen Systems als Ursache consecutiver Herzerkrenkungen. Klin. Med. Wohnschr. 5: 361,1868 .

283. Porter, K.R. and P. Vanamee. Observations on the formation of connective tissue fibers. Proc. Soc. Exptl. Eiol Med. 71: 513, 1949.

284. Prasad, B.N. The mechanical activity of gut muscle under anaerobic conditions. J. Physiol. 855: 249, 1935.

285. Prosser, C.L. and F.A. Brown. Comoarative Animal Physiolony, 2nd edition. Philadelphia: W.B. Saunders, 1961. 240 p.

286. Prosser, C.L., G. Burnstock, and J. Kahn. Conduction in smooth muscle: Comparative structural properties. Am. J. Physiol. 199: 545, 1960.

287. Ramachandran, G.N. and G. Kartha. Structure of collagen. Nature 174: 269, 1954.

288. Ramachandran, G.N. and G. Kartha. Structure of collagen. Nature 176: $593,1955$.

289. Ramsey, R.W. and S.F. Street. The isometric lengthtension diagram of isolated skeletal muscle fibers of the frog. J. Cell. Comp. Physiol. 15: 11, 1940.

290. Redenz, E. Untersuchungen Ulber die elastische Faser. I. Untersuchung des isolierten elastischen Faser des Nackenbandes mit dem Mikromanipulator. Beitr. Pathol. Anat. U. Allgen. Paithol. 76: 226, 1926.

291. Remington, J.W. Hystersis loop behavior of the aorta and other extensibile tissues. Am. J. Physiol. 180: 83, 1955.

292. Remington, J.W. Extensibility behavior and hystersis phenomena in smooth muscle tissues. In Tissue Elasticity. J.W. Rerington, ed. Washington, D.C.: Am. Physiol. Soc., 1957, p. 138.

293. Remington, J.W. Pressure-diameter relations of the in vivo aorta. Am. J. Physiol. 203: 440, 1962. 
294. Remington, J.W. The physiology of the aorta and major arteries. Handbook of Physiolooy, Section II, Vol II, Chapter 24. Baltimora: Williams and Wilkins Co., 1963. p.799.

295. Remington, J.W., W.F. Hamilton, and P. Dous. Some difficulties involved in the prediction of the stroke volume from the pulse wave velocity. Am. J. Physiol. 144: 536,1945 .

296. Reuterwall, O.P. Úber die Elastizitat der Geflyswllnde und die Methode ihrer naheren Prúfung. Acta. Med. Scand. (Suppl. 2): 1, 1921.

297. Rhodin, J. and N.T. Dalham. Electronmicroscopy of collagen and elastin in lamina propria of the tracheal mucosa of rat. Exp. Cell Res. 9: 371, 1955.

298. Rhodin, J.A.G. Fine structure of vascular walls in mammals. Physiol. Rev. (Suppl. 5) 42: 48, 1962.

299. Roach, M.R. and A.C. Burton. The reason for the shape of the distensibility curves of arteries. Can. J. Biochem. Physiol. 35: 681, 1957.

300. Roach, M.R. and A.C. Burton. The effect of age on the elasticity of human arteries. Can. J. Biochem. Physiol. 37: 557, 1959.

301. Roddie, I.C. The transmembrane potentials associated with smooth muscle activity in turtle arteries and veins. J. Physiol. 163: 138, 1962 .

302. Rouse, H. Fluid Mechanics for Hydraulic Engineers. Now York: McGrau-Hill, 1938.

303. Roy, C.S. The elastic properties of the arterial wall. J. Physiol. 3: 125, 1880.

304. RUlegg, J.C., E. Strassner, and R.H. Schirmer. Extraktion und Reinigung von Arterien-Actomyosin, Actin and Extraglobulin. Biochem. 2. 343: 70, 1965.

305. Samarasinghe, D.D. An electron microscope study of the arteries to the brain of the rat with special roference to the accompanying norve fibers. J. Anat. 97: $311,1963$. 
306. Sandow, A. Latency relaxation: A brief analytical revieus. Med. Coll. Virginia Quart. 2: 82, 1966.

307. Sarnoff, S.J. and E. Berglund. Pressure-volume characteristics and stress relaxation in the pulmonary vascular bed of the dog. Am. J. Physiol. 171: 238, 1952.

308. Saxton, J.A., Jr. Elastic properties of the rabbit aorta in relation to ag日. Arch. Path. 34: 262, 1942.

309. Scarselli, V. Increase in elastin content of the human aorta during growth. Nature 191: 710, 1961.

310. Schlichter, J.G. The vascularization of the aorta. Am. J. Med. Sci. 218: 610, 1949.

311. Schmiterlow, C.G. The nature and occurence of pressor and depressor substances in extracts from blood vessels. Acta Physiol. Scand. (Suppl.) 16: 56, 1948 .

312. Schmitt, 8.0 . and J. Gross. Further progrees in the electronmicroscopy of collagen. J. Am. Leath. Chem. Assoc. 43: 658, 1948 .

313. Schmitt, F.O., C.E. Hall, and M.A. Jakus. Electron microscopy investigations of the structure of collagen. J. Cell. Comp. Physiol. 20: 11, 1942.

314. Schoenberg, C.F. Electron microscope study of smooth muscle in pregnant uterus of the rabbit. J. Biophys. Biochem. Cytol. 生 609, 1958.

315. Schoenberg, C.F., J.C. Rüegg, D.M. Needham, R.H. Schirmer, and $H$. Nemetchek-Gansler. A biochemical and electron microscope study of the contractile proteins in vertebrate smooth muscle. Biochem. Ztschr. 345: 255., 1966 .

316. Shimamoto, T. The relationship of edematous reaction in arteries to atherosclerosis and thrombosis. J. Atheroscler. Res. 3: 87, 1961. 

317. Siegel, S. Nonnarametric Statistics. Now York: MicGraw-

318. Siggins, G.R. and H.J. Berman. Intracellular potentials in arteriolar smooth muscle. Physiologist 10: $305,1967$.

319. Smith. C., M.M. Seitner, and H-P. Wang. Aging changes in the tunica media of the aorta. Anat. Rec. 109: 13, 1951.

320. Smith, S.G., E.A. Davidson, and R.L. Hill. Composition of normal and pathological cutaneous elastin. Nature 197: 1108, 1963.

321. Sonnenblick, F.H., D. Spiro, and H.M. Spotnitz. Ultrastructural basis of Starling's Law of the Heart. The role of the sarcomere in determining ventricular size and stroke volume. Am. Heart. J. 68: 335, 1964.

322. Sparks, H.V., Jr. and D.F. Bohr. Effect of stretch on passive tension and contractility of isolated vascular smooth muscle. Am. J. Physiol. 202: 835, 1962.

323. Speden, R.N. The effect of initial strip length on the noradrenaline-induced isometric contraction of arterial strips. J. Physiol. 154: 15, 1960.

324. Spiro, D. and E.H. Sonnenblick. Comparison of the ultrastructural basis of the contractile process in heart and skeletal muscle. Circ. Res. (Suppl. 2): $14 ; 1964$.

325. Starcher, B., C.H. Hill, and G. Matrone. Importance of dietary copper in the formation of aortic elastin. J. Nutrition 82 : $318,1964$.

326. Stearns, M.L. Studies on the development of connective tissue in transparant chambers in the rabbit's ear. Am. J. Anat. 67: 55, 1940.

327. Strasburger, J. Ueber den Einfluss der Aortenelastizität auf das Verhaltnis zurschen Pulsdruck und Schlagvolumen des Herzens. Plunch. Med. Woschr. 54: 1022, 1907. 
328. Strong, K.C. A study of the structure of the media of the distributing arteries by the method of microdissection. Anat. Rec. 12: 151, 1938.

329. Su, C., J.A. Bevan, and R.C. Ursillo. Electrical quiescence of pulmonary artery smooth muscle during sympathomimetic stimulation. Circ. Res. 15: 20, 1964.

330. Sudhof, H. Uber den Kohlenhydzatstoffwechsel der Arterienwand. Pflug. Arch. ges. Physiol. 252: 551, 1950.

331. Thomas, J., D.F. Elsden and S.M. Partridge. Partial structure of tuso major degradation products from elastin. Nature 200: 651, 1963.

332. Tickner, E.G. The static elastic behavior of excised arteries. Proc. 18th Ann. Conf. on Engineering. Med. Biol. I: 31, 1965.

333. Torrance, H.B. and S. Shwatz. The elastic behavior of the arterial wall. J. Roy. Coll. Surg. Edinburgh ?: $55,1961$.

334. Tunbridge, R.E., R.N. Tattersall, D.A. Hall, W.T. Astbury, and $R$. Reed. The fibrous structure of normal and abnormal skin. Clin. Sci. 13: 315, 1952.

335. Uchida, E., D.F. Bohr, and S.W. Hoobler. A method for studying isolated resistance vessels from rabbit mesentery and brain and their responses to drugs. Circ. Res. 21: 525, 1967.

336. Umbreit, W.W., R.H. Burris, and J.F. Stauffer. Manometric Techniques and Tissue Metabolism, 3rd edition. minneapolis: Burgess, 1957. p.149.

337. Usuku, G. Electronmicroscopical studies on the ultrastructure of the elastic fiber. I. Electronmicroscopical

... observation of bovine ligamentum nuchae in ultrathin sections after potassium permanganate fixation. Kumamoto Med. J. 11: 84, 1958.

338, Van Citters, R.L., B.M. Wagner, and R.F. Rushmer. Architecture of small arteries during vasoconstriction. Circ. Res. 10: 668, 1962. 
339. Verzar, F. Das Altern des Kollagens. Helv. Physiol. Pharm. Acta 14: 207, 1956.

340. Vio, A., G. Cozzetti, A. Reggioni, and A. Platania. On the distribution of the vasa vasorum in the main arteries and veins. An experimental study on the normal dog. Angiologica 1: 357, 1964.

341. Walker, S.m. Potentiation and hysteresis induced by stretch and subsequent release of papillary muscle. of the dog. Am. J. Physiol. 198: 519, 1960.

342. Wartman, W.B. Vascularization and hemorrhage in the arterial wall. Studies in Pathology, presented to Peter MacCallum. Melbourña: Melbourne Univ. Press, 1950.

343. Wasserman. F., L.E. Roth, and O.T. Minick. The fine structure of native collagon in thin sections. Exptl. Cell. Res. 13: 407, 1957.

344. Waugh, W.H. Adrenergic stimulation of depolarized arterial muscle. Circ. Res. 11: 264, 1962.

345. Weckman, N. Local constriction and spasm of large arteries elicited by hypothalamic stimulation. Experientia 15: $34,1960$.

346. Weinstein, H.J. and P.H. Ralph. Myofilaments from smooth muscle. Proc. Soc. Exp. Biol. Med. 78: 614, 1951.

347. Weissman, N., G.S. Shields, and H.W. Carnes. Cardio-vascular studies on copper deficient swine, IV. J. Biol. Chem. 238: 3115, 1963.

348. Wezler, $K$. and A. Boger. Die Feststellung und Beurteilung des Elastizitat zenraler und peripherer Arterien am Lebenden. Arch. Exptl. Path. U. Pharm. 180: 381, 1936.

349. Wiggers, C.J. and R. Wegria. Active changes in size and distensibility of the aorta during acute hypertension. Am. J. Physiol. 124: 603, 1938.

350. Wilens, S.L. The postmortem elasticity of the adult human aorta. Its relation to age and to the distribution of intimal atheromas. Am. J. Physiol. 13: 811, 1937. 
351. Wilens, S.L. and R.T. McCluskey. The comparative filtration properties of excised arteries and veins. Am. J. Med. Sci. 224: 540, 1952.

352. Winton, F.R. The influence of length on the response of unstriated muscle to electrical and chemical stimulation and stretching. J. Physiol. 61: 368, 1926.

353. Woorner, C.A. Endothelial reaction to intravenous lipids. Anat. Rec. 109: 360, 1951.

354. Wohlisch, E. Die thermischen Eigenschaften der faserig structurierten Gebilde des tierischen Bewegungsapparates. Ergeb. Physiol. 34: 406, 1932.

355. Wohlisch, E., H. Weitnauer, W. Grunig, and R. Rohrbach. Thermodynamische Analyse der Dehning des elastischen Gewebes von Standpunkt der statistisch-kinetischen Theorie der Kantschukelastizitat. Kolloid Z. 104: $14,1943$.

356. Wolinsky, $H_{\text {. }}$ and 5. Glagov. Structural basis for the static mechanical properties of the aortic media. Circ. Res. 14: 400, 1964.

357. Wolfe, D.F., L.T. Potter, K.C. Richardson, and J. Axelrod. Localizing tritiated norepinephrine in sympathetic axons by electronmicroscopic autoradiography. Science 138: 440, 1962.

358. Wolpers, C. Das Scheiben- und das Lamellenstadium der Kollagengnerstriefang. Makrowol. Chem. 2: 37, 1948.

359. Wood, G.C. Some tonsile properties of elastic tissue. Biochem. et Biophys. Acta 15: 311, 1954.

360. Woodside, G.R. and A.J. Dalton. The ultrastructure of lung tissue from newborn and embryo mice. J. Ultrastruct. Res. 2: 28, 1958 .

361. Yamamoto, T. Electron microscope investigation on the relationship between the smooth muscle cell of the Proc. vermiformis and the autonomic peripheral nerves. Acta Neurovegetativa 21: 406, 1960. 
362. Yanoff, H.M., A.L. Rosen, N.M. MeDonal'd, and D.A.incDonald. A critical study of the response of monometers to forced oscillations. Physics Med. \& Biol. ㅁ: 407, 1963.

363. Yardley, J.H., M.W. Heaton, L.m. Gaines, Jr., and L.E. Shulman. Collagen formation by fibroblasts. Johns Hopkins Hosp. Bull. 106: 381, 1960.

364. Yater, W.M. and I.W. Birkeland. Elasticity (extensibility) of the aorta of human beings. Am. Heart J. 5: 781, 1930 .

365. Yokota, H. Histochemical and electronmicroscopical studies of elastic fibers. I. Electronmicroscopical observation of bovine ligamentum nuchae in ultrathin sections after elastase treatment. Kumamoto Med. J. 10: 25,1957 .

366. Zatzman, M., R.W. Stacy, J. Randall, and A. Eberstein. Time course of siress relaxation in arterial segments. Am. J. Physiol. 177: 299, 1954.

367. Zelander, T., R. Ekholm, and Y. Edlund. The ultrastructural organization of the rat exocrine pancreas. III. Intralobular vessels and nerves. J. UItrastruc. Res. I: 84, 1962. 
APPROVAL SHEET

The dissertation submitted by Philip Boone Dobrin has been read and approved by five members of the faculty of the Graduate School.

The final copies have been examined by the director of the -dissertation and the signature which appears below verifies the fact that any necessary changes have been incorporated, and that the dissertation is now given final approval with reference to content, form and mechanical accuracy.

The dissertation is therefore accepted in partial furlfillment of the requirements for the Degree of Doctor of Philospry.
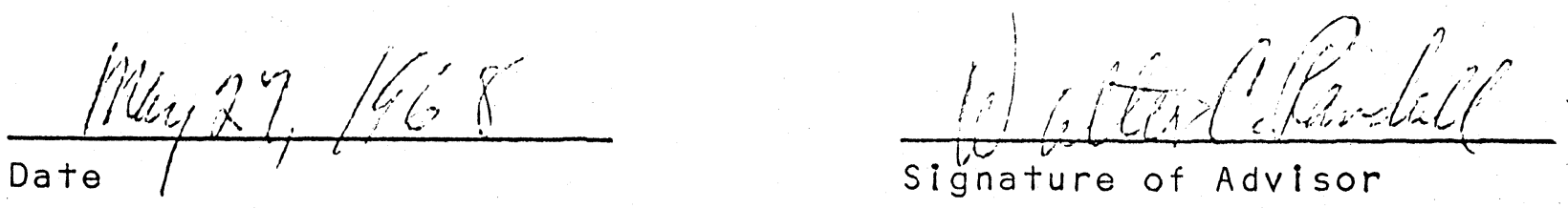